\title{
The Rhetoric of Investment Theory
}

The Story of Statistics and Predictability 
ISBN 9789462954229

Copyright (C) 2015, Thomas Pistorius

Printed by Uitgeverij BOXPress / Proefschriftmaken.nl, 's-Hertogenbosch

All rights reserved. No part of this book may be reproduced, in any form or by any means, without permission in writing from the author. 


\section{The Rhetoric of Investment Theory}

The Story of Statistics and Predictability

\section{De retoriek van de beleggingstheorie}

Het verhaal over statistiek en voorspelbaarheid

\section{Proefschrift}

ter verkrijging van de graad van doctor aan de

Erasmus Universiteit Rotterdam

op gezag van de rector magnificus

Prof.dr. H.A.P. Pols

en volgens besluit van het College voor Promoties.

De openbare verdediging zal plaatsvinden op donderdag, 14 januari 2016 om 11.30 uur

door

\section{Thomas Pistorius}

geboren te Dongen 


\section{Promotiecommissie}

Promotor:

Prof.dr. S.J. Magala

Overige leden:

Prof.dr. A. de Jong

Prof.dr. A. Klamer

Prof.dr. T.P. Kocken 


\section{Contents}

$\begin{array}{lr}\text { Chapter 1: Introduction } & 1\end{array}$

1.1: The Assumption of Predictability and its Relevance.........................................

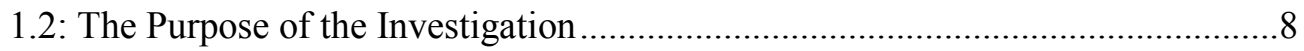

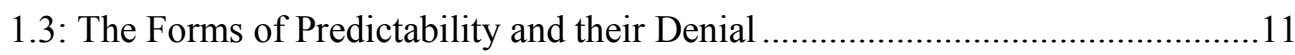

1.4: The History of Modern Investment Theory, Chapter 2 …...............................17

1.5: The Theories of Probability and Uncertainty, Chapter 3.................................18

1.6: The Rhetoric of Economics, Chapter 4 ..........................................................20

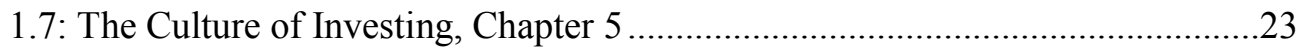

Chapter 2: The History of Investment Theory 25

2.1: An Introduction to the History of Investment Theory ......................................25

2.2: Finance in Europe in the $13^{\text {th }}$ to $18^{\text {th }}$ Century ..................................................34

2.3: Efficient Market Theorists in the $19^{\text {th }}$ and Early $20^{\text {th }}$ Century ..........................39

2.4: Finance in the First Half of the $20^{\text {th }}$ Century ...................................................45

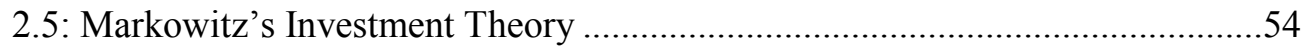

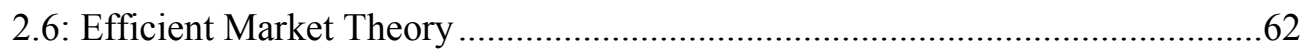

2.7: CAPM

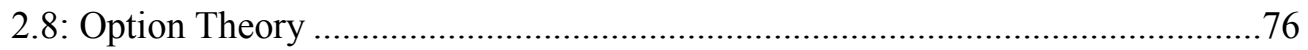

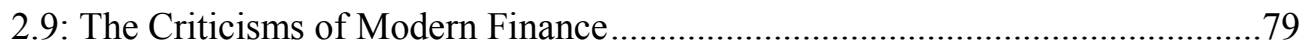

Appendix 2A: The Mathematical Statistics of Diversification.................................99

Appendix 2B: The Black \& Scholes Option Formula ..........................................105 


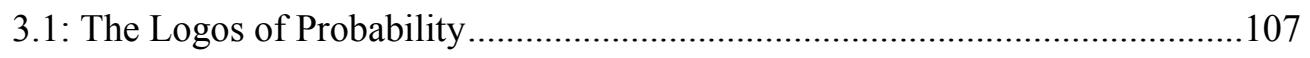

3.2: Probability Beliefs in Portfolio Theory ……….............................................108

3.3: Markowitz's Defence of Personal Probabilities .............................................113

3.4: Investment Theory after Markowitz's Portfolio Theory ..................................116

3.5: Evaluation of Probability Theory in Investment Theory ................................117

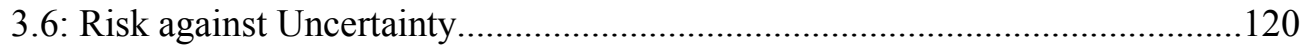

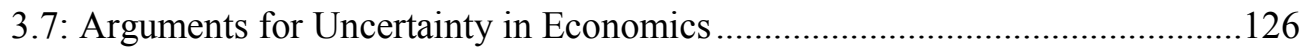

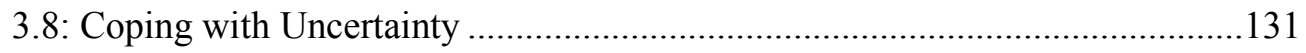

3.9: Implications of Uncertainty for Investment Theory .....................................139

3.10: A Thought Experiment with Predictability in Investment Theory .................143

3.11: Closing Remarks about the Thought Experiment.........................................155

Appendix 3A: A Formal Proof of the Thought Experiment....................................157

Chapter 4: Beyond Statistics: A New Rhetoric for Investment Theory 161

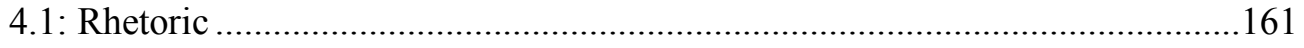

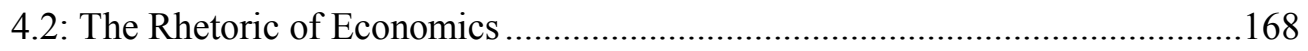

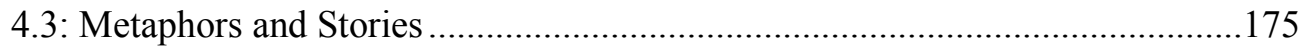

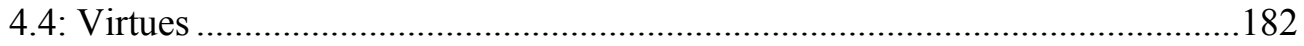




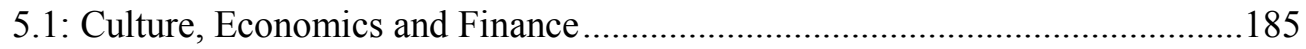

5.2: Values, Decision Making and Phronesis ......................................................192

5.3: Methodology of the Investigation of the Culture of Investing ........................196

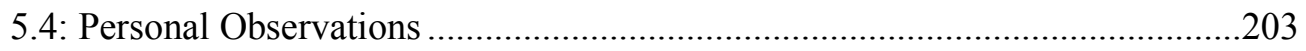

5.5: Literature on the Culture of Investing ........................................................208

5.6: Innovative Case 1 The Management of Investment Risk.............................213

5.7: Innovative Case 2 Shell's Scenarios-approach..............................................223

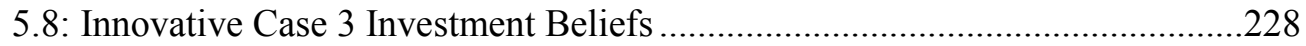

5.9: Values, Conversations, their Justification, and Innovation ...........................233

Chapter 6: Conclusions $\quad 241$

$\begin{array}{lr}\text { Works Cited } & 249\end{array}$

$\begin{array}{lr}\text { Acknowledgements } & 277\end{array}$

$\begin{array}{lr}\text { Summary } & 279\end{array}$

$\begin{array}{ll}\text { Samenvatting } & 281\end{array}$

$\begin{array}{lr}\text { About the Author } & 283\end{array}$ 



\section{Chapter 1}

\section{Introduction}

"How dreadful!" cried Lord Henry. "I can stand brute force, but brute reason is quite unbearable. There is something unfair about its use. It is hitting below the intellect (Oscar Wilde, 1891)."

\section{1: The Assumption of Predictability and its Relevance}

The Assumption of Predictability in Investing

Predictions pervade economics and investing in its theory and practice.

Specifically in investment theory and its practice, stochastical predictions dominate. A stochastical prediction is a prediction of an average outcome with a probability distribution attached. But do statistics predict in investing? Is it, to paraphrase Oscar Wilde (1891), un-fair to use the brute reason of statistics for investing if statistics cannot stand the test of the intellect?

Besides its mere intellectual relevance, the assumption of predictability is relevant for academia in economics and investing because the opposite case of unpredictability and its consequences are outside the mainstream conversation and therefore need attention. From a societal point of view, the assumption of prediction in economics and investing has consequences for the economy, the financial wealth and well-being of people, witness the consequences of the credit crisis of 2008. Practice based on investment theory means that if the theory fails, the risk management fails as well, and, that the investment portfolios do not deliver a sufficient return, meaning that the clients will suffer from lower than expected wealth in investments and pensions. The general public also suffers from failing financial risk management indirectly: in the United States, the credit crisis of 2008 and its consequences caused an conservatively estimated loss of foregone 
economic growth of 40 to 90 percent of a one year's GDP, or \$6 to 14 trillion, which equals $\$ 50,000$ to $\$ 120,000$ for every household in the United States (Atkinson, Luttrell \& Rosenblum, 2013).

The discussion of the received view of predictability and its related idea of rational markets, is also relevant for the financial services industry, their supervisors such as central banks, and the policy makers. The financial services industry can improve their risk management and their long term added value for clients, which both help the continuity of their businesses in the long run. Central banks and policy makers should include unpredictability and irrationality in their policies of supporting trust, stability, and healthy growth, by distrusting low volatility, booms in the stock and housing markets, and, so-called optimistic new era thinking about the future of the economy (Shiller, 2000), as a reason for loosening restrictions on lending of banks, borrowing of households, and regulations and capital requirements for the financial sector.

\section{The Queen and Economic Prediction}

After the credit crisis in 2008 Queen Elizabeth asked an economist "Why did no one see the crisis coming (Skidelsky, 2009b)?”. The Post Keynesian Robert Skidelsky argues that the question makes sense, because economics presents itself as a predictive discipline like a natural science. To elaborate on the Queen's point, economics, besides its theoretical purpose, indeed serves the practical purpose of decision making: therefore individuals and the society should understand whether economics predicts, or which parts of it predict, and to what extent the prediction is merely general, or meant to be specific. To make sure, our society perceives economics as a predictive discipline. Philip Mirowski (2013, p. 246 and further) distinguishes three reasons for the perception that economists should predict:

1. Milton Friedman's methodological paper (1953) proposed the goal of prediction for economics, 
2. a lot of economists have made a living out of predicting, and,

3. finance and economics made prediction since the 1980 s central to the theory, among other things founded on the rational expectations theory.

But economics resembles other social sciences, and does not predict either: "It is only by imagining a mechanical world of interacting robots that economics has gained its status as a hard, predictive science (Skidelsky, 2009b)." Skidelsky believes that the credit crisis has been aggravated as a consequence of the intellectual failure of the dominant neoclassical school of economics, which assumes a stable world with rational agents and efficient use of information (Skidelsky, 2009a). In the credit crisis, the ideology of the rational market as advocated by Alan Greenspan and others was put to a reality check (Fox, 2009). The ideology of rational markets, which implies that the market comes up with the true price, makes one forget that the financial market is "a devilish thing" as well (Fox, 2009, p. xv).

How statistics interprets data of the financial markets can also offer an explanation for the credit crisis of 2008. The assumptions behind the models in finance seem to imply that risk is manageable, but turbulence is normal, not abnormal, in financial markets (Mandelbrot \& Hudson, 2004). If turbulence in financial markets is normal, modern finance is problematic indeed, in Mandelbrot's \& Hudson's words:

Financial economics, as a discipline, is where chemistry was in the sixteenth century: a messy compendium of proven know-how, misty folk wisdom, unexamined assumptions and grandiose speculation (Mandelbrot \& Hudson, 2008/2004, p. xv).

In line with the ideas of Mandelbrot, Taleb (2010) explains how investment theory is a cause of the credit crisis of 2008 because it neglects the consequences of low probability events, aka (also known as) black swans. 


\section{The Problem with Prediction in Investment Theory}

Modern investment theory assumes that future expected returns and standard deviations are predictive. Markowitz $(1952,1959)$, generally perceived as the father of modern investment theory, leaves no doubt that he believes in the predictability of investment returns in the long run. Investment theory after Markowitz's contribution $(1952,1959)$ evolves on mathematical statistics: though the investment theory of equilibrium on financial markets (Sharpe, 1964) and its empirically based successors differ from Markowitz's approach, the investment decision still takes the statistical form of an expected return and a risk in the form of a standard deviation. The practices of financial risk management and the composition of investment portfolios in the financial sector have evolved out of modern investment theory (MacKenzie, 2006).

Because academic investment theory also constitutes practice, the validity of predictability by statistics determines the sense making of decision-making by investment theory. If we assume predictability by statistics as valid, then statistics rules investment theory and its practice. Yet, if the arguments in the dissertation persuade that investment theory based on statistics does not predict, the theory is meant to support decision making. Once more, the credit crisis of 2008 may clarify the sense making of investment theory in investment practice. In the aftermath of 2008, an investor typically could have asked his investment manager:

In the crash on the financial markets in 2008, the MSCI World, the global stock market index, dropped $38 \%$. If you compare the expected maximal loss as indicated by the investment model before 2008, with the realized loss in 2008, a gap emerges. So, our model before 2008, based on investment theory, was wide of the mark. Is the investment model still correct? 
The investment manager could have answered in two ways:

1. Yes and no. No, because the investment model failed in the crisis of 2008 . Yes, because in the meantime we improved the model: the improved model approximates the future reality of financial markets.

2. The question assumes that the investment model delivers real predictions of risk and return. Yet, we use the investment model merely to support decisions on investment policy, but not to predict.

Translated into the language of philosophy, the investment manager has answered:

1. The models of investment theory are based on scientific laws, and therefore predict. Thus investment models result in truth, in the sense of the correspondence theory of truth. The correspondence theory says that a proposition is true, if it corresponds to facts (Audi, 1995). The proposed risk and return parameters of the investment model correspond to the 'factual', objective future reality.

2. The outcome of the investment model interprets the future. The model produces fiction, not truth, unless by coincidence. The investment model enables the investment manager to get to grips with the future, and enriches judgment with historical and theoretical insights.

I believe that answer 1 is wrong. Answer 2 is possibly correct, and originates from rational behaviour, instead of predictability. What rationality means will be an important theme in the dissertation.

The Relevance for Academia, Witness the Textbooks 
The case for unpredictability in investment theory is usually no topic in the field's textbooks, in its literature, or in its university courses. The broader literature on investment theory does not debate the possibility of unpredictability, because unpredictability is the opposite of the ideas of neoclassical economics. Indeed, the field of modern investment theory seems to ignore the history of economics and finance, and current non-mainstream schools of economics and finance, a reason why the dissertation will discuss both items. We will now have a look at what the textbooks of investment theory tell us about predictability.

A textbook expresses the normal science of a field (Kuhn, 1996/1962, p. 137 and further). A scientific community founds its practise on normal science. To illustrate the ideas about the predictability of investment returns by statistics, I will examine a number of textbooks about investment theory. A simple test is whether the textbooks refer to Knight (1921), because Knight distinguishes risk and uncertainty: risk is stochastically predictable, uncertainty does not provide predictability. The textbooks that I examined were a part of my education as a MSc in finance and a financial analyst in the post-doctoral VBA-education. The VBA-education is the Dutch equivalent of the CFA-education. None of the five textbooks in my sample refer to Knight (Bernstein \& Damodaran, 1998, Bodie, Kane \& Markus, 1989, Maginn \& Tuttle, 1983, Reilly, 1994, Solnik, 1996). The textbooks neither refer to other writers on uncertainty in economics such as Keynes (1936, 1937) and Von Mises (1949). The five textbooks seem to suggest that the probability distribution of returns can be estimated in a reasonable way. The result, that the textbooks in my sample do not mention uncertainty, was to be expected because the idea of being able to estimate probability distributions underlies economics and investment theory. I do not intend to disqualify the high level of the textbooks mentioned as the books elaborate in depth on the paradigm of modern investment theory - though I wish to see the paradigm evaluated because, again, it has proven to be harmful in practice if not fully understood because the risks of financial markets can be underestimated. Though in my opinion the 
textbooks should incorporate uncertainty, one cannot blame the textbooks for their one-sidedness, because learning necessarily 'indoctrinates' since in order to learn one has to take basic assumptions for granted (Feyerabend, 2010/1975, p. 8).

\section{The Relevance for Society, Witness Political Economics and Political Finance}

Predictability in economics and financial markets relates to the domain of political economics as well. In the socialist calculation debate, Ludwig Von Mises and Friedrich Hayek disputed with proponents of the socialist economic model whether economics is able to calculate optimal outcomes for a socialist economy (Rothbard, 1991). The proponents of a socialist economy won the theoretical part of the debate, because the techniques for neoclassical economics can be used for a socialist economy as well: if predictability in economics is a proper assumption, both the market and the socialist economy function optimally in theory. The conclusion of the theoretical part of the socialist calculation debate confronts one with the absurd consequences of the assumption of predictability: both a market and a socialist economy are optimal in theory despite their incompatible ideas about the role of markets and the state in the economy.

Thomas Piketty (2014) combines predictability of financial markets with the political economic topic of income and wealth inequality. Though he warns that the causes of future inequality cannot be reduced to economic mechanisms (Piketty, 2014, p. 20), he nevertheless proposes that inequality will grow, because the return on financial assets of the richest $1 \%$, outpace economic growth (ibid., pp. 25-26). He founds his pre-diction on historical data, and thus supposes that the historical return is representative of the future return. My criticism on Piketty's view on future inequality is that if the return on the financial markets would be specifiable above some rate above the risk-free rate, all we would have to do to diminish inequality is to lend the other $99 \%$ of the people money to the buy stocks and other financial assets. If the assumption of Piketty that the future return equals the historic return is true, the result should be a sure gain for the $99 \%$, if in practice 
there would be enough financial assets available to be bought. The assumption that financial assets yield a specifiable return above the risk-free rate sounds too good to be true, and cannot be true, which I will try to underpin in the dissertation. Furthermore, common-sense tells us that financial assets risk more than risk-free assets, so Piketty's prediction is too simple, because it does not take risk into account.

Another actual account within political finance is David Graeber's (2011) history about the nature of debt and its ethical side. To him, debt expresses a social relation in which debt is a promise of which the repayment depends on power relations: to the powerless, debt is presented as a moral obligation, the powerful on the other hand are not held to the moral obligation to redeem. The credit crisis in 2008 illustrates Graeber's point: the losses of the financial sector were socialized as the government, i.e. the tax payer, paid the bill, seemingly leaving the people responsible people in the financial sector relatively undamaged. Graeber (2012/2011, p. 46) regards money as a form of debt as well. If money is debt, then money does not need to possess intrinsic value, unlike in the form of a silver or golden coin. Graeber's historical account of the term 'stock' is interesting for investing. The term 'stock' comes from the $12^{\text {th }}$ century custom in England to notch the level of debt onto tally sticks, which represented the 'stock' for the debtor. The term 'stock holder' originates from the custom. Analogue to his political account of debt and money, it seems to me that one could perceive stocks as postulated in modern investment theory, as a power relation in the form of a promise of a specified return above the risk-free rate and a specified risk.

\section{2: The Purpose of the Investigation}

The idea of stochastical prediction seems to be taken for granted in mainstream investment theory. But stochastical prediction is a problematic assumption of investment theory. As I will underpin in the dissertation, stochastical prediction is 
a subtle notion which must be based on solid arguments, which cannot be found in the textbooks. Because of the reflective nature of the dissertation, rhetoric, history, philosophy, and culture form the major reference to discuss the foundations of stochastical predictability in investment theory.

The central question of the dissertation is: What grounds the use of stochastical predictability in investment theory?

The central question of the dissertation is investigated by a number of sub questions:

1. What is the rhetoric of investment theory?

The rhetoric of investment theory translates into questions as: what are the rational arguments (logos), constitutional ideas (metaphors), and the discourse of investment theory? The analysis of the rhetoric of investment theory is grounded on the approach of the rhetoric of economics by McCloskey. The rhetoric of investment theory is under-researched. The investigation of the rhetoric of investment theory is enriched by the economic theories of uncertainty, the history of finance, alternative investment theories, the philosophy of probability, the culture of investing, and innovative practices.

2. What philosophy of statistics is applied in investment theory?

The arguments for assuming stochastical predictability are founded on statistics, which is a theory of how to interpret randomness. The various theories of statistics have implicit assumptions about the structure of reality, in other words, the theories of statistics have different philosophies of probability. 
3. What are the arguments for uncertainty as the opposite of predictability? Non-mainstream economics assumes unpredictability instead of predictability and has heavily debated predictability and statistics. Their arguments against predictability and statistics are an important source for making the case against predictability.

4. Do alternative investment theories offer a better explanation, modelling, prediction or handling method?

The history of investment theory and current alternative investment theories offer various paradigms for investment theory which do not claim to predict but focus on explanation or alternative modelling. It is important to show the alternative non-mainstream investment theories because they contrast and compete with the received view of investment theory.

5. Can virtue and value ethics compensate the assumed epistemological deficiencies of investment theory in decision making?

If the epistemology of mainstream and alternative investment theories is not suited for predicting, practical reason in the form of virtue or value ethics can become relevant, also because the ethics in economics and investment theory is reduced to merely the rationality of prudence.

6. What explanations offers an investigation of the culture of investing for the use of investment theory?

As an extension of rhetoric, the perspective of culture offers a fertile ground for explaining and exploring the behaviour of investors. I will follow Klamer's approach of the culture of economics because is relevant and applicable to investing. Also innovative practices within the culture of investing will be considered. 
The purpose of the dissertation is to investigate stochastical predictability in investment theory by an extensive reflection from rhetorical analysis, uncertainty economics, philosophy of statistics, history, culture, innovative practices, and literature on alternative investment theory.

\section{3: The Forms of Predictability and their Denial}

In the introductory Chapter, a clarification of the notion of predictability and its counterarguments are now essential for understanding the discussion forthcoming: what forms of predictability exist, which notions of prediction are applied in investment theory, and, what are the main arguments of unpredictability in economics and investment theory? In Markowitz's investment theory, predictability is to be understood as stochastical predictability. Stochastical predictability differs from forecasting: a stochastical prediction explicates probabilities, a forecast does not. Forecasting delivers a number, or a range between two numbers, for example of some future price or economic indicator. A stochastical prediction yields a probability distribution, meaning a range of outcomes with probabilities attached. The dissertation is about the case for using statistics in investing, not merely about forecasting, though we will see in Knight's thinking (1921) in Chapter 3 that if probabilities and outcomes are uncertain, meaning non-measurable, stochastical probability and forecasting resemble.

\section{The Forms of Predictability}

Let us analyse what kind of predictions exist. The first form of prediction is by a deterministic law in which a causal law extrapolates the present conditions to its future conditions (Audi, 2006/1995, p. 124). A deterministic law also shows the specific path to a future state. An example of a deterministic law from classical mechanics is the calculation of the place of landing of a cannon ball. The deterministic law relates to an underlying stable structure which enables the prediction. In economics, price theory provides a good example of the appliance of 
deterministic laws, for example, how demand for goods decrease after its price increases.

The second form of predicting is by a teleological law in which predictability is possible because an end state is known (Audi, 2006/1995, p. 124). Now, the starting point matters less and the path towards to the end state can vary as well. In physics, the teleological law is illustrated by the experiment in which a ball reaches a state of equilibrium at the lowest point of a bowl. In investment theory, the equilibrium theories of valuation (Graham et al, 1934) (Williams, 1938) and the Capital Asset Pricing Model (CAPM) (Sharpe, 1964) are such teleological theories. The theory of valuation assumes that the price of a security will tend to its intrinsic, equilibrium, value. In the CAPM, the continuation of Markowitz's portfolio theory, informational efficiency ensures an equilibrium. The equilibrium relates to an underlying stable structure.

Regularity is the third form of prediction. Regularity assumes that the future resembles the past. The analogy that future cases equal past ones assumes stability. A prediction of regularity is that the sun will rise tomorrow, because it did so in the past. Of course, a prediction of regularity, like the daily sun rise, is upgraded to a causal prediction if a more specific scientific theory is available. Yet, in more complex cases, like in economic phenomena, multi-causality denies simple causal predictions.

The three forms of prediction, causal, teleological and by regularity, can have the form of a deterministic prediction, meaning 'having one outcome', or a stochastical prediction, meaning 'having more than one outcome'. To repeat, a stochastical prediction results in a number of outcomes with probabilities attached. An example of a stochastical prediction is the outcome of the rolling of a dice. The structure of the dice causes the outcomes 1 to 6 to appear in equal quantities at a large number of throws. The certainty of stochastical predictions restricts to 
artefacts such as dices, or in economics to actuarial affairs, in which stability for some period can be assumed (Knight, 1921). To fully profit from stochastical predictability, one has to have access to the results of the whole group of outcomes. The teleological theories of investing, valuation theory as used in Markowitz's portfolio theory and the CAPM, have an evident stochastical nature: risk accompanies the equilibrium value of the CAPM, in valuation theory it is uncertain when the price of a security will equal its fundamental value. Predicting regularities by past data is done in empirical investment theory. The frequency theory of statistics provides the apparatus for predicting regularities by past data. The frequency theory of statistics also uses the term predictability to indicate whether a sample can predict some outcome representative for a population: the use of the term prediction stems from the acceptance of the assumptions of the frequency theory of statistics and the particular probability distribution applied, the stability of the distribution in the future, and its method of testing. In the dissertation will be argumented that, whereas artificial probabilities yield certainty over the outcomes of a group of cases, past observations of financial markets in general do not possess the apodictic quality needed for stochastical prediction.

\section{Profitable versus Unprofitable Predictability}

Mainstream economics believes in profitable predictability, which is disputed from within economics:

The best economic scientists, of whatever school, have never believed in profitable casting of the fores (McCloskey, 1990, p. 109).

In the dissertation profitable predictability is relevant; of course, economics delivers all kinds of non-profitable predictions, as well as general economic principles. An example of a non-profitable prediction is the certainty of the result of the mechanism of interest rate parity by risk-free arbitrage. General economic principles like in price theory predict on a more general, non-specific, level. The 
same kind of non-profitable predictability exists for investing: for example that a period of rising stock prices will be followed by a period of falling prices, that high levels of valuation will be followed by lower levels of valuation, that more risk is rewarded by a higher return.

\section{The Case for Deterministic Unpredictability in Economics}

After having discussed the forms of predictability, I will now discuss the denial of predictability in economics and investment theory. Concerning the denial of predictability in economics, my introduction focusses on the ideas of Frank Knight (1921), who is one the most important thinkers about uncertainty in economics. Knight clarifies the assumptions of neoclassical economics, and concludes the unpredictability of most economic phenomena. Knight reflects in the classic Risk, Uncertainty and Profits (1921) on the price theory of perfect competition, a cornerstone of neoclassical economics. The mechanics part of physics has been the model for economics in the price theory of perfect competition; both are small but founding parts of physics and economics:

An abstract deductive system is only one small division of the great domain of economic science, but there is opportunity and the greatest necessity for cultivating that field. Indeed, in our analogy, theoretical mechanics is a very small section of the science of physical nature; but it is a very fundamental section [...] (Knight, 2009/1921, p. 2).

Yet, the analytical method of physics is effective because few and important common elements dominate:

The laws of these few elements, therefore, enable us to reach an approximation to the law of the situation as a whole. They give us statements of what 'tends' to hold true or 'would' hold true under 'ideal' conditions, meaning merely in a situation where the numerous and variable but less important 'other things' which our laws do not take into account were entirely absent (ibid, p. 1). 
The analytical method in physics works in practice because its models are approximately true: the laws of physics allow us to build a bridge and to put people on the moon. But the analogy with physics does not hold for economics: Knight argues that price theory, and most of economics, has complicated causes, unlike mechanics in which a few common elements dominate. An extended account of the analogy of physics and economics would be out of the scope here, and can be found in for example Mirowski (1989). Knight claims that economics along the lines of the method of physics is speculative and dangerous, because theorists and practitioners tend to forget that the assumptions are necessarily too simple:

$[\ldots]$ the allowances and corrections necessary in the case of theoretical economics are vastly greater than in the case of mechanics, and the importance of not losing sight of them is correspondingly accentuated. The general principles do not bring us so close to reality; there is a larger proportion of factors in an economic situation which are of the variable and fluctuating sort (ibid, p. 5).

To clarify the difference between the outcomes of mechanics and price theory in practice, Knight investigates the postulates of price theory. His investigation addresses uncertainty as the reason why the outcomes of the price theory of perfect competition do not match the outcomes in practice: in theory, profit should not arise in a perfect competition, but it does in practice because of uncertainty. Though Knight's ideas about uncertainty in economics remain influential, uncertainty never became leading in economics as Wubben (1993) shows in an investigation of the history of uncertainty in economics:

In tracing trends in the treatment of uncertainty in neo-classical economics, following its introduction into economics by Knight, we have found two major trends. First, during the interwar period the Knightian concept of uncertainty was first of all restricted to the domain of profit theory; later, it became a side issue; and finally it was shunted into a scientific railway siding (Wubben, 1993, p. 53). 
Knight's ideas about uncertainty in economics and his investigation of price theory contribute to the critical investigation of investment theory and will be explained in detail in Chapter 3 of the dissertation.

Knight's criticism of economics does not necessarily imply a criticism of idealized theory as such. Economics in the form of pure theory has a purpose as well: a utopian theory informs us about the difference between the theory and the world, and thereby enhances our understanding of the world. For example, the price theory of perfect competition gave Knight the contrast to express his ideas about uncertainty in economic phenomena. In neoclassical economics and investment theory, the theory takes the form of a model. The nature of models is an important subject in the philosophy of science. A model is a simplification and, usually, an idealization of a phenomenon (Reiss, 2013, p. 119). The purpose of a model can be to explain the causal mechanism behind a phenomenon, or to predict. It makes sense to model economic phenomena: the mathematics used in the models naturally invites us to be a more precise about the economic mechanisms involved. The models of economics and investment theory differ in character: some models, like the interest rate parity, do explain and predict like physical mechanics, yet, to repeat, Knight's criticism stresses that models in economics usually lack dominant explaining features and therefore have to cope with multi-causality.

\section{The Case for Stochastical Unpredictability in Investment Theory}

Let us now contrast the case for stochastical predictability in investment theory, whether teleological or by regularity, with that for unpredictability. The argument for unpredictability is grounded in the instability of the probability distribution of investment returns. The cause of instability is the unpredictable nature of the changes in the substrate which 'produces' investment returns. Unpredictable changes happen in, for example, the structure of the economy, society, environment, investor attitudes, or in expectations about them. Of course, the historical investment returns technically add up to some probability distribution: 
but the distribution should be qualitatively judged as representative or nonrepresentative for the future. Future stability or instability of a probability distribution is a matter of belief, which is to be justified by arguments. The argument about future instability or stability of investment returns cannot be settled by the statistical method, because the argument lies outside the scope of statistics. Prediction in statistics, and in science in general, is grounded on stability, not on instability. A nuance needs to be made regarding the stability assumption, because some economic phenomena are more stable than others: say, the sales of foodstuffs is more stable than the sales of chemicals or steel which tend to depend on the economic cycle: the key is that one needs arguments for stability, its determinants, and its expected duration.

\section{4: The History of Modern Investment Theory, Chapter 2}

Investment theory is nowadays regarded as a part of economics, though in Chapter 2 about the history of investment theory will be demonstrated that investment theory as a part of finance has a separate history, and is only since the 1960s regarded as economics, that is financial economics. As is customary in academic finance, finance is used in the dissertation to denote investment theory as well. The sociology of science explains the promotion of the new scientific movement of finance since the 1960s. Modern finance had to be 'new' because of its new paradigms. Yet, the history of finance as described in the Chapter 2 starts from the beginning of the $13^{\text {th }}$ century, when Fibonacci published his Liber Abaci. The roots of finance since the $13^{\text {th }}$ century probably emerged from actuarial science and probability theory. The importance of actuarial science for finance is that it relates to the valuation of options, which was needed to solve the practical problem of the value of a life annuity. In France of the 1860s the theory of behavior of financial markets takes the step from manipulation and bubbles to the efficient market theory. The first half of the $20^{\text {th }}$ century is full of interesting research on finance. 
Current mainstream investment theory is founded on Markowitz's portfolio theory $(1952,1959)$. Portfolio theory aims to enable an investor to compose a statistically legitimate portfolio of individual stocks, bonds, and other assets. After Markowitz determined how a rational investor would act, the next logical step in financial economics was a formulation of the market equilibrium of expected risk and return. So, for example, William Sharpe (1964) builds a theory of equilibrium in investment markets, $a k a$ the Capital Asset Pricing Model (CAPM). Another continuation of the CAPM is an empirical branch in investment theory, which tries to identify anomalies in the efficient market hypotheses (Robert Haugen, 1995). Option theory is also a part of modern investment theory (Black \& Scholes, 1973).

Besides the history of modern investment theory, Chapter 2 critically reviews modern investment theory and shows the alternative investment theories. The ideological criticism of modern finance, political finance, uses elements of behavioural and bubble criticisms, and combines them with the criticism on free markets in general. Mandelbrot provides within the rational mathematical tradition an alternative statistical theory, which explains seemingly predictive patterns in financial markets, wild volatility, and bubbles. Bubble theory explains bubbles on financial markets and their bursting. Behavioural finance is also insightful and helps to reflect on decision making, and explains behaviour of market participants. The bottom-up approach of evolutionary finance yields an interesting alternative to the top-down approach of modern investment theory.

\section{5: The Theories of Probability and Uncertainty, Chapter 3}

Chapter 3 explains the foundations of probability theory as applied in investment theory, and discusses the arguments against predictability and statistics of Knight (1921), Keynes (1936, 1937), Von Mises (1949), McCloskey (1990) en (Taleb, 2007). The arguments for unpredictability in economics and investing all stem from a lack of future information and the denial of the mainstream statistical 
theory. Chapter 3 also presents a thought experiment about stochastical predictability in investment theory. The possible new argument discusses the idea of predictability by the rational mechanism of risk-free arbitrage: there cannot actually be stocks of the kind that investment theory postulates.

Let us now introduce the two theories of probability used in mainstream economics and investment theory with a citation of the Post Keynesian Paul Davidson:

\section{The objective probability environment}

Decision makers believe that the past is a statistically reliable, and hence unbiased, guide to the future. This is the rational expectations hypothesis, where knowledge regarding future consequences of today's decisions involves a confluence of subjective and objective probabilities.

\section{The subjective probability environment}

In the individual's mind, subjective (or what Savage calls personal) probabilities regarding future prospects at the moment of choice govern future outcomes. These subjective probabilities need not coincide with objective distributions, even if well-defined objective distributions happen to exist [...] (Davidson, 1991, pp. 130-131, his insertion).

The objective, frequentist approach regards the probability of an event, as the relative frequency in past observations: the approach is the statistics often used in empirical science. The subjective, or personal approach, uses the machinery of probability to explicit beliefs, but mixes with the probabilities of the objective approaches as well (Savage, 1954). Leonard Savage (1954) claims that the use of personal probabilities is consistent with rational behaviour, which has been heavily debated in the economic literature. The notion of rationality needs careful attention in investment theory, and will be treated at length in dissertation. 


\section{6: The Rhetoric of Economics, Chapter 4}

In Chapter 4 of the dissertation, I will present a rhetorical analysis of predictability in investment theory along the lines of McCloskey's project of the rhetoric of economics. Rhetoric is a way of reflecting on science, like sociology and philosophy of science. McCloskey pioneered the analysis of the rhetoric of economics (1983, 1985a, 1990, 1994, 1996, and 1998). Her analysis of rhetoric combines the techniques of classical rhetoric with those of literary criticism, the latter being a $20^{\text {th }}$ century theory of rhetoric. With literary criticism she bridges the gap between literature and science (1990, p. 30, my insertion): “The scientific report is itself a [literary] genre, whose conventions have changed from time to time."

To gain an understanding of McCloskey's rhetorical approach, I will first explicate her ideas about economics as a science in general. Despite being a critic of economics, she considers economics as the queen of social sciences (1996). Economics is about prudence, which is an important virtue in human action. Economics is successful as a historical science, but not as a predictive one in the sense that it delivers easy profits (1998). In her words:

Economics is a sort of social history. For all the brave talk about being the physicists of the social sciences, economists do their best work when looking backwards, the way a paleobiologist or geologist or historian does (McCloskey, 1990, p. 31).

Besides being a historical science, economics is a theoretical one as well. The value of pure economic theory is that it gains insights into the crucial assumptions in economic phenomena. The mathematics used in the models merely explicates relationships between economic variables and clarifies the working of the economic mechanism. When working at the University of Chicago, McCloskey 
wrote The Applied Theory of Price (1985b), about applying the mechanisms of price theory: she has arguably believes in the usefulness of applied mathematical models, which matter for the purpose of explanation and non-profitable general prediction.

To clarify what 'rhetoric' means in McCloskey's project of the rhetoric of economics, it is necessary to distinguish between the phenomenon of rhetoric (its practice), and the discipline of rhetoric (its theory). One can distinguish two meanings of the phenomenon, and two of the discipline:

1. the phenomenon of rhetoric with a negative connotation

2. the phenomenon of rhetoric in a neutral sense

3. the discipline of rhetoric for the creation of rhetoric

4. the discipline of rhetoric for the analysis of rhetoric

'Rhetoric' as the phenomenon in the negative sense means hollow speech, i.e. speech which merely aims at persuasion by arousing the emotions of the audience. The popular use of the term 'rhetoric' as hollow speech is often associated with politicians. Rhetoric in the negative sense can also mean flowery speech. In a neutral sense, the phenomenon of rhetoric signifies that every argument, good or bad, consists of rhetorical elements which aim to persuade. The discipline of rhetoric applies to both its creation and its analysis. McCloskey investigates the rhetoric of economics as a neutral phenomenon. With the discipline of rhetoric she analyses the phenomenon of the rhetoric of economics. I will do the same for stochastical predictability in investment theory. Rhetorical criticism sensitizes us to the possibility that a writer, possibly unconsciously, tries to make us look in a particular way. Her project is to analyse the phenomenon of rhetoric by means of rhetoric as a discipline. 
To get an introductory impression of the discipline of rhetoric, I will briefly present the ideas of Aristotle (2006). Aristotle is regarded as having developed the first full theory of rhetoric and defined rhetoric as $(2006, \S$ I.i.12.2): “[ $[.$.$] the faculty$ of discovering the possible means of persuasion in reference to any subject whatever." Yet, the brief description of Aristotle cannot arguably represent the full scope of rhetoric. In contemporary usage the possible range of meanings of rhetoric is even wider than in the classical use and incorporates for example discourse, literary criticism, and theories of discourse or language. In $A$ Companion to Greek Rhetoric its editor lists the meanings of rhetoric as employed by its contributors:

[...] one can discern the word 'rhetoric' or 'rhetorical' being used to denote a wide range of phenomena, including oratory, parts of speech, prose genres, figurative language, performance, pedagogical practices, discourse, the strategic use of language, persuasion, and various theories of discourse, language or persuasion. [....]. Notably, there are a goodly number of other disciplinary terms that are just as broad in scope, including anthropology, sociology, psychology, and politics (Worthington, 2010/2007, p. 4, his italics).

McCloskey's rhetorical investigations have a wide reach as well. The investigations are about style and the implicit or explicit reasons for the particular style employed. They also cover the analysis of the metaphors, the arguments, the narratives, the methodology, the philosophy, the science and the discourse of economics. Chapters 2 and 3 will focus on the logos, the rational arguments, of stochastical predictability in investing by close reading. To gain a deeper understanding, I will focus in Chapter 4 at the metaphors, the models, of investment theory, as well as the discourse of investment theory, and what needs to be changed in the discourse, among other things by the inclusion of virtue ethics. To my knowledge, no specific literature exists on the rhetorical analysis of the use of statistics in investment theory. 


\section{7: The Culture of Investing, Chapter 5}

In Chapter 5, I work along the lines of Klamer's (2001, 2003, 2006, 2007, 2014) approach of the investigation of the culture of economics, which also suits with investment theory as a part of economics, that is financial economics. The analysis of culture helps to explain the behaviour of investors. The central claim of the dissertation that predictability does not apply to investing, raises the questions why investors hold on to their practices of prediction, and what constitutes the practices of investors, seen through the perspective of culture.

Klamer's cultural approach fits in with the rhetorical approach applied in the dissertation, which has been Klamer's point of departure as well (1988 \& 1992 \& 1995 with McCloskey, 1994 with Leonard, 2001). Although a conversation resembles rhetoric, Klamer (2007) prefers conversation because it relates to a community and its cooperative character. Within the cultural approach Klamer concentrates on conversations and values. By relating culture to economics, Klamer (2014) poses a question in the Weberian tradition of distinguishing between procedural and substantive rationality. Economics is about procedural rationality, culture is about substantive rationality. Both substantive rationality and culture centre at values, or, the answer to 'What is important?'.

The field of culture also relates to uncertainty. Hofstede (1997) has investigated cultural differences between countries in the research project for IBM. The way a culture handles uncertainty is a part in Hofstede's explanatory model. To him, uncertainty is a feeling of anxiety and as a matter of culture, uncertainty avoidance begs for predictive ability by some method. Using a predictive method such as stochastical prediction handles uncertainty by replacing the feeling of uncertainty with a rational, technical method. Thus, risk per se is not the problem of uncertainty: the ambiguity of uncertainty is the reason of anxiety. Ambiguity aversion is also a subject of behavioural finance. 
Chapter 5 also investigates three innovative practices in the Dutch institutional investment world by interviews with their proponents and their literature. The alternative approaches are about 'the management of investment risk', 'the Shell scenarios approach', and 'investment beliefs'.

Chapter 6 presents the conclusions of the dissertation. 


\section{Chapter 2}

\section{The History of Investment Theory}

"History [...] could produce a decisive transformation in the image of science by which we are now possessed (Kuhn, 1996/1962, p. 1)."

\section{1: An Introduction to the History of Investment Theory}

\section{History Enriches}

The study of the history of investment theory enhances the insight into the rhetoric of modern investment theory, its underlying philosophy, and its culture, and thereby enriches the dissertation. At the same time, the Chapter serves to explain modern investment theory by studying its seminal texts and treating its rivals. Thomas Kuhn (1962), a historian, philosopher, and sociologist of science, advocates the study of the history of a scientific field. An analysis of the history of a field clarifies its current paradigms and contrasts them with the previous and the competing paradigms (Kuhn, 1962). His approach implies that science can have incompatible theories, and does not label earlier theories as unscientific (Kuhn, 1996/1962, p. 2). Yet, the textbooks of a field present the image of science that Kuhn opposes: "Inevitably [...] the aim of such books is persuasive and pedagogic [...] (ibid., p. 1)." Textbooks are merely meant to explain the leading paradigms of a field, are unhistorical by nature and suggest that science is accumulated: “Textbooks thus begin by truncating the scientists's sense of his discipline's history and then proceed to supply a substitute for what they have eliminated (ibid., p. 137)." In their purpose, textbooks merely refer to the work and persons that easily can be seen as a part of the leading paradigm; the unhistorical practice of textbooks is not constrained to the scientific community "The temptation to write history backward is both omnipresent and perennial (ibid., p. 138).” 
Kuhn's historical approach highlights that a dogmatic scientific methodology is a constraint on answering scientific questions (ibid., p. 3). In his perspective, science is a way of seeing, which is arbitrarily mixed by personal and historical elements, and grounds on a set of received beliefs (ibid., p. 4). The study of the history of science as proposed by Kuhn does not yield the correct theory, but it helps understanding the sociology of scientists, how they operate, and provides a broader view on the historically competing currents within a field. The discussion in the Chapter stems from three sources: the available research into the history of investment theory, the philosophical, critical, reading of a number of seminal texts, and the evaluation of the competing investment theories. The philosophical reading of seminal texts and the evaluation of competing theories is, of course, targeted beyond the purpose of a textbook.

Because investment theory is a part of finance, the term 'finance' is now clarified. The broad term 'finance' can mean:

1. The practice of manipulating and managing money.

2. The capital involved in a project, especially the capital that has to be raised to start a new business.

3. A loan of money for a particular purpose, especially by a finance house.

4. An academic discipline within the general field of economics dealing with funding, financial markets, and the funding implications for managing businesses (Law, 2014, keyword 'finance').

The fourth meaning of 'an academic discipline' is relevant here. Finance as an academic discipline can be divided into two subdisciplines. The "economics dealing with funding $[\ldots]$ and the funding implications for managing businesses (ibid., 2014)" belong to the subdiscipline of corporate finance. The remaining part of the fourth meaning of finance that deals with financial markets is the subdiscipline of investment theory. Corporate finance and investment theory differ 
from perspective. Corporate finance takes the perspective of the organisation central, while investment theory focusses on the investor in financial markets. The theories of corporate finance and investment theory overlap. As is customary in academic finance, finance is used here to denote investment theory as well. Financial economics is another name for finance because modern finance has been applying price theory, a cornerstone of neoclassical economics.

Finance and economics have had separate histories. In the fifties, Milton Friedman has denied investment theory as a part of economics during Markowitz's defence of his dissertation on investment theory (Markowitz, 1990, p. 286). Though Friedman could not remember the remark later on, he nevertheless could agree to it, because Markowitz's theory was indeed about applied mathematics, and not about economics (MacKenzie, 2008/2006, p. 50). Finance and economics as separate fields, was no strange idea in the $1950 \mathrm{~s}$, because the fields have different roots. Finance and economics have usually been taught at different institutions: finance has been a subject at the business schools, and economics has been thought at the universities (MacKenzie, 2008/2006, p. 5). The business schools were "[...] use-oriented and populated by a faculty of practitioners, operating at the margins of university academia (Poitras, 2007, p. 7)." Yet, institutional developments have been of importance to the evolution of finance (Poitras, 2007). Since the 1960s, business schools have often become a part of universities and their intellectual norms. Since then, the evolution of finance has resembled that of mainstream economics; like economics, finance has changed its institutional account for a modernistic one. In the twentieth century, economics as a mathematical, statistical, and theoretical science has succeeded to economics of an institutional sort, which was of a historical, qualitative and measurement driven nature. Financial economics is an offspring of modernistic economics (ibid.).

Since the 1960s, finance has become incorporated in economics and few would probably argue now against the inclusion of finance in economics. Modern finance 
is grounded on the theory of full competition, the cornerstone of price theory. Because of arbitrage under full competition, the price of a good tends to its cost price. Full competition in finance means for the Capital Asset Pricing Model (CAPM), the equilibrium model of financial markets, that the risk and return of securities will be balanced. Yet, a financial market differs from a goods market because expectations can be priced instantaneously in a financial market. Immediate processing of information is usually not a feature in a goods market, because the competitors need time to adapt their production levels, which after a time lag feed back into the price of a good. Therefore, financial markets stand as a perfect model for other markets.

In its canonical history, modern investment theory is founded on Markowitz's portfolio theory $(1952,1959)$. Markowitz constructed his portfolio theory on statistics. Portfolio theory intends to compose a statistically legitimate portfolio. The CAPM, invented by Sharpe (1964) and others, generalizes the individual behaviour of the 'Markowitz'-investor to an equilibrium in financial markets. The equilibrium model is founded on the efficient market hypothesis, which means that investors cannot beat the market because all available information has been incorporated in the market prices. The efficient market hypothesis in its elaborated form is invented by Samuelson (1965b) and Fama (1965a, 1965b). Markowitz's mean-variance approach, the efficient market hypothesis, and the CAPM constitute the core of modern portfolio theory (Poitras, 2007, p. 5). Efficient market theory also results in the option theory of Black \& Scholes (1973) and Merton (1973b), another core of modern investment theory. Option theory treats the valuation of a contingent claim, a claim of which the outcome depends on an uncertain phenomenon, such as the future price of a stock.

\section{The Pitfalls of History}

We have to be careful, however, to perceive finance as a new science. The sociology of science (Kuhn, 1962) explains the reason for the promotion of the new 
scientific movement of finance since the 1960s. Modern finance had to be 'new' because of its new paradigms:

Because modern financial economics provides a textbook case of these processes [i.e. the processes dominated by sociological factors], it is necessary to disentangle the essential intellectual contributions from those that have gained attention due to the 'techniques of the huckster' involving 'repetition, inflated claims and disproportionate emphases' (Poitras, 2007, pp. 1-2, my insertion).

Because of education, the promotion of modern finance as a new science has been successful. Usually, the authority argument of academic education persuades the student of a simplified history of a field:

One can speculate about how an academic field could so distort its own origins. Its history is largely rewritten, as it were, by the victors. New students too often rely on the version of scholarly history conveyed to them by their mentors, who themselves are too dependent on their mentors, and so forth (Rubinstein, 2006a, p. xii).

Illustrated by the literature, it is easy to find leading theorists who emphasize the scientific movement of finance since the 1960s, for example Robert Merton who claims:

The Modigliani-Miller work stands as the watershed between 'old finance', an essentially loose connection of beliefs based on accounting practices, rules of thumb and anecdotes, and modern financial economics, with its rigorous mathematical theories and carefully documented empirical studies (Merton, R.C., 1987, "In Honor of Nobel Laureate, Franco Modigliani", Journal of Economic Perspectives, Vol. 1 No. 2, pp. 145-155, in Poitras, 2007, p. 2).

Yet, 'old' finance, finance before the 1960s seems mathematically rigorous as well, and has carefully documented empirical studies. For example, Bachelier's random walk theory (1900) or Williams's valuation models (1938) are mathematically rigorous, and many of its empirical studies are documented, such as for example Cowles $(1933,1944)$ and Working (1934) about the unpredictability of the stock 
market. The 'watershed' mentioned by Merton in the previous citation, has made the old finance appear less valuable, and therefore less cited by the new finance (Poitras, 2007, p. 3).

It might not be surprising that the history of finance has been ignored as a consequence of the construction by the scientific movement of modern finance. So, the notion that finance was not scientific before the 1960s, seemed to be taken for granted until the 1990s, when the history of finance started to be investigated:

[...] the academic community of modern financial economics only arose during the 1960s. One of its major preoccupations was the creation of a canonical history, which glorified its past (Jovanovic, 2006b, p. 211).

Until the 1990s, the canonical history of modern financial economics provided what was known about the construction of this discipline. As with any canonical history, it was a means to structure and to legitimize a scientific discipline (ibid., p. 191).

Contrary to the canonical version of modern finance, finance has deep historical roots, and had been thoroughly developed before the 1960s (Poitras, 1996, 2006, 2007). Finance in the $20^{\text {th }}$ century before the 1960 s, was concerned with accounting (financial statements), legal issues (securities law), and institutional issues, topics which are still relevant today (Poitras, 2006, p. 1). From the 'old' finance also stem the theories of security analysis and technical analysis, which are still being practiced today. Another topic which was highly developed before the 1960s is the analysis of bonds as illustrated by the work of Frederick Macaulay (1938) (Poitras, 2007). Macaulay's work will not be treated here, because the analysis of bonds is outside the scope of the dissertation.

A documented start of finance as the technique of providing loans and calculating interest, can be traced back to around 2000 BC in Babylonia, but arguably finance existed before that time as well (Goetzmann \& Rouwenhorst, 2005, pp. 3-4). 
Because the scope of the Chapter is mostly confined to the history of stochastical predictability for finance, the history of finance begins at the start of the $13^{\text {th }}$ century, when Fibonacci published his Liber Abaci (translated as 'The book of calculations') in 1202. In the work of Fibonacci is for example described how to calculate a net present value, a cornerstone of finance (Goetzmann, 2005, p. 123). Finance since the $13^{\text {th }}$ century is grounded on actuarial science and probability theory: the confluence of finance and economics in the $20^{\text {th }}$ century is more recent in historical terms (Poitras, 1997).

A question relevant to the history of science is which authors should get the credit for its main ideas. Inevitably, investigations into history often result in incomplete findings, our knowledge of history is necessarily incomplete. Even if all the required historical sources would be available, it would take a long time before the sources would be scrutinized and well understood. Research into the history of science has showed that authors often anticipate, or independently formulate, the same scientific ideas:

In most of these cases, the individuals commonly given bibliographical credit in academic papers were actually anticipated many years, occasionally decades or centuries, earlier. In some cases, there were others with independent and nearsimultaneous discoveries who are seldom, if ever, mentioned, offering one of many proofs of Stephen Stigler's law of eponymy that scientific ideas are never named after their original discoverer! This includes Stigler's law itself [...] (Rubinstein, 2006a, p. xii).

The scope of the current Chapter is, of course, not aimed at performing research into old and new primary sources. But I will consult different sources of historical research into finance, in order to compose a history of finance for the purpose of the dissertation. The authors of historical research consulted here are, among others, Peter Bernstein (1992, 1996, 2007), Geoffrey Poitras (1996, 2006, 2007), Colin Read (2013), and Mark Rubinstein (2006a). The investigations into the history of finance reveal a number of authors, for example Louis Bachelier (1900) 
and Benoit Mandelbrot (1963a, 1963b, 2004 with Hudson), who are absent in the picture sketched by the textbooks of finance:

Intellectual history is replete with examples of individuals making contributions that were seminal in content but, by and large, did not receive the corresponding recognition and attention (Poitras, 2006, p. 5).

\section{The Assumption of (un-)Predictability}

Of importance to the dissertation is what the history of finance and economics tells about the predictability of markets. Besides the theoretical importance, the predictability of risk and return of investments matters for practice. As Bernstein says, all investors agree that it is hard to get rich by investing:

Yet, in the face of admittedly high odds, enough people do try to predict stock prices to keep an entire industry humming (Bernstein, 2005/1992, p. 17).

The history of finance and economics tells two tales about predicting: one tale tells that we cannot predict, and the other tale tells we can predict. The efficient market theory claims that predicting, in the sense of beating the market, is not possible. Though, the CAPM grounded on the efficient market theory, claims that a stochastical prediction of risk and return is possible. Yet, a number of theories in finance and economics explicitly says that predicting beyond beating the market is impossible as well, see for example Knight (1921), Keynes (1936, 1937), Von Mises (1948) or Mandelbrot (1963a, 1963b, 2004 with Hudson).

The tale in finance in which predicting, in the sense of beating the market is possible, has many expressions, such as the theories of valuation, the Dow-theory, and theories about exploitable anomalies of the efficient markets. The theories of the valuation of securities imply that if the price of a security on the market deviates from its intrinsic value, arbitrage opportunities arise (Graham et al, 1934, Williams, 1938). The Dow-theory, a chartist theory, is an alternative theory of prediction on the basis of historical patterns of prices on the financial markets. To 
summarize, the views on predictability in financial markets have, by closer inspection, three variations:

1. the theories that believe in beating the market index

2. the CAPM, which includes the efficient market theory, does not believe in beating the market, but believes in stochastical predictability of risk and return.

3. the theories of Knight, Keynes, Von Mises, and Mandelbrot which deny any stochastical and other forms of predictability in financial markets.

I like to establish that my dissertation which denies stochastical predictability of investments, is a logical extension of modern finance, with its denial that predictions can outperform the market. I do, however, not believe that market efficiency implies stochastical predictability of risk and return of investments; the impossibility of stochastical predictability I will discuss at length in Chapter 3. Then, I will also discuss the notion of uncertainty which is the epistemological transitional phase between stochastical predictability and total uncertainty, in which judgment, instead of predictability, is central (Knight, 1921). Judgment under uncertainty takes many forms, such as subjective probability, econometric analysis, qualitative opinion, ordinal probability, or a general prediction.

In the current Chapter, modern portfolio theory receives a large part of the attention besides the historical investigations. Other relevant underpinning investment theories are treated, such as the valuation of securities and option theory. A number of alternative investment theories will be treated as well:

- Keynes's theories of the beauty contest, 'efficient market', and animal spirits

- the quantitative empirical based theories of non-efficiency in markets

- investing as gambling 
- the alternative theory of Mandelbrot based on a non-normal probability distribution

- bubble theory

- behavioural finance

- evolutionary finance.

\section{2: Finance in Europe in the $13^{\text {th }}$ to $18^{\text {th }}$ Century}

Finance as the technique of supplying loans is as old as antiquity (Poitras, 2006, p. 2). The oldest evidence of financial contracts stems from $2400 \mathrm{BC}$ from the southern part of Mesopotamia, Babylonia, in the form of short-term loans (Van de Mieroop, 2005, p. 20). For reasons of scope, our historical discussion of finance begins in Europe at the beginning of the thirteenth century. Finance since the thirteenth century, concentrates on the analytical problems of discounting future cash flows and the valuation of contingent claims.

An important condition for the development of finance is the numerical notation. Fibonacci, or Leonardo da Piso (1170-1250) introduces the numerical notation from the Middle East to Europe by his Liber Abaci, meaning 'The book of calculations', published in 1202 (Rubinstein, 2006a, p. 3). Fibonacci elaborates the numerical notation to the finance problems of that time, such as the distribution of profit in partnerships, and the discounting and accruing of interest:

Fibonacci illustrates his methods of calculation through several numerical examples. Among these are four types of applications to investments: (1) the fair allocation of profits to members of a partnership ('On Companies,' [...]; (2) the calculation of profits from a sequence of investments, with intermediate withdrawals ('Problems of Travelers,' [...]; (3) the calculation of future value ('A Noteworthy Problem on a Man Exchanging One Hundred Pounds at Some Banking House for Interest,' [...]; and (4) the calculation of present value ('On a Soldier Receiving Three Hundred Bezants for His Fief,' [...]) (Rubinstein, 2006a, p. 4). 
Finance before the Enlightenment must be considered from its ethical and theological context, in which usury and gambling were prohibited (Poitras, 2006, pp. 2-3). Usury means that it is forbidden to charge interest on loans. To illustrate its importance, the forbidding of usury is a theme in the work of the scholastic philosopher Thomas Aquinas (1225-1274) (Sylla, 2006, p. 18). A common division in the stages of the forbidding and reassessment of usury, is the period of 1150-1450, in which the scholastic theory of usury applies, and the period of 14501750 , in which the scholastic theory of usury is reassessed; yet, the common division underplays that ideas about the loosening of usury were expressed in the first period of 1150-1450 as well (ibid., pp. 18-19).

In business practice, usury had to be avoided because it was prohibited in canon law. But the interpretations of canon law allowed interest to be paid in concealed forms (Poitras, 1996, p. 4). Eventually, the insight grew that usury and payment of interest were two separate things: usury overcharges borrowers who are in need of money, but payment of interest compensates the lender for the opportunity cost of providing money. That lending money bears the opportunity cost of missed profits, was witnessed by the daily commercial practice (Sylla, 2006, p. 19).

In the 15th century the loosening on usury and gambling, enabled the development of pricing securities. The reckoning masters, or algorists, who had adopted the Indian-Arabic calculation methods such as presented in the Liber Abaci, were specialized in the theory pricing of securities. An example of a master algorist is Nicholas Chuquet (1445-1500), who illustrates in Triparty en la science des nombres (1484) the arithmetic available at that time (Poitras, 1996, p. 5).

Eventually, the theory about the pricing of financial assets further expands, among other things because of the growth and acceptance of commercial activity and the reformation (ibid., p. 5). The development in finance was directed at the valuation of the then existing securities. Valuation fulfilled a practical need because it serves as a calculation of the correct, fair price of a financial asset. At that time, questions 
of valuation arose for bonds, annuities, life contingent claims, and partnerships in business.

An example of such an obligation is a census, paying annually and backed by land or future taxes, which evolved into an annuity (Poitras, 1996, p. 5). As early as in the thirteenth century, the city of Venice issues a census, which becomes securitized in the $14^{\text {th }}$ century. In the $16^{\text {th }}$ century, the market for loans develops and the need emerges to compare the loans with the help of valuation theories (ibid., p. 6). The calculation of compound interest is also needed, which was at that time allowed by canon law because religious tolerance of commercial activity arose. In the second part of the $17^{\text {th }}$ century more complex fixed income securities with redeemability, sinking funds, and lottery elements, are followed by elaborated valuation techniques (ibid., p. 8).

Though usury prohibits loans with interest in the Middle Ages, partnerships of business in which profits and losses are shared, are customary (Sylla, 2006, p. 14). Relevant questions of finance are at that time how to divide profits between partners with different roles and within different timeframes. Thus, finance answers the question what is a fair probability behind a Rawlsian veil of ignorance, when dividing profits in the partnership of a business (ibid., p. 12). So, pre-modern probability theory is grounded on contractual or institutional grounds, and not on the later adopted physical grounds of frequency theory (ibid., p. 13). When dividing profit among a fixed group of partners, such an ethical perspective of fairness on probability is appropriate.

The history of actuarial science is also relevant for finance because it encompasses the valuation of options. Both actuarial science and finance involve in the pricing of contingent claims with risk neutral valuation. Probability theory is needed to solve the relevant practical problem of the valuation of a life annuity: 
Not only were these securities important in state and municipal finance in France, England and Holland; in an era pre-dating actuarially sound pension plans and life insurance the life annuity performed an essential social function (Poitras, 2006, p. 79).

Because solving the problem of the valuation of a life annuity was hard, scholars from outside the commercial arithmetic practice were attracted. Without the intention of being complete, I will illustrate a number of important contributors to solving the problem of the valuation of a life annuity. Among the first to support is the mathematician Simon Stevin (1548-1620), who contributed to, for example, the tables of the present value of annuities (Poitras, 1996, p. 16). The Dutch statesman Johan de Witt (1625-1672) thinks up an analytical solution to the price of a life annuity by involving the probability of dying, which is a novelty (Poitras, 2006, p. 84). After De Witt, Edmond Halley (1656-1742), famous for his work on comets, provides an improved solution to the valuation of a life annuity (ibid., pp. 87-88). Abraham de Moivre (1667-1754) completes the theory of life annuities by solving the theoretical gaps in the valuation of life annuities (Poitras, 1996, p. 20).

Since Abraham de Moivre's approach of frequency theory in 1730, the medieval approach of ethical probabilities becomes out of sight. Blaise Pascal and Pierre de Fermat discover modern probability theory in the second half of the 17th century, followed in the first half of the eighteenth century by De Moivre, who proposes the normal or bell-shaped curve and the dispersion measure of the standard deviation, and by Daniel Bernoulli who invents decision theory (Bernstein, 1996, pp. 4-5). The probability theory of Pascal, De Fermat, De Moivre, and Bernoulli still grounds finance today:

[...] the tools we use today in risk management and in the analysis of decisions and choice [...] stem from the developments that took place between 1654 and 1760 [...] (Bernstein, 1996, pp. 5-6).

Stocks such as we are now familiar with, emerge in the beginning of the $16^{\text {th }}$ century. In the 16th century, the economic focus shifts from Southern to Northern 
Europa. At that time, Antwerp, Amsterdam and London develop exchanges for commodities and securities, which include derivatives such as forwards and options (Poitras, 1996, p. 6). After 1620, the publicly traded securities of the jointstock companies emerge. A joint-stock company has two distinct features in comparison to a private stock: a joint-stock is transferable and has a limited liability (ibid., p. 22). Because the volume of joint-stocks and government debt expands, in 1695 some 100 joint-stocks are traded in London, security markets become necessary (ibid., p. 9).

Amsterdam precedes London in having a major modern security market. The first stock traded on the Amsterdam Exchange in 1602 is the Dutch East India Company. The share trading practices in the beginning of the 17th century in Amsterdam are marked by the manipulation of the stock market (Van Dillen, Poitras and Majithia, 2006, p. 45). Joseph de la Vega (1650-1692) writes an eyewitness report of the stock market of Amsterdam. The title of De la Vega's book, Confusion de Confusiones (1688), to be translated as 'The confusion of confusions', refers to the darkness of the workings of the stock market. Amsterdam is then the prime financial centre of the world (Cardoso, 2006, p. 64). The tulip mania from 1634 to 1637 in Amsterdam accentuates that speculation is a part of the era (ibid., p. 65). De la Vega's book is set up as a dialogue between a philosopher, a merchant and a stockholder. De la Vega claims that, despite that speculators and gamblers play a big part in the stock market, the valuation of a stocks is based on fundamentals as well (Poitras, 1996, p. 23). Cardoso concludes about De la Vega's book that it was meant to show that investing in the stock market was worthwhile:

[...] there should be no dispute about the main purpose of Joseph de la Vega when writing his book: to demonstrate that, though the inner or potential risks and dangers of financial operations, the dealings at the stock exchange market were worth pursuing (Cardoso, 2006, p. 73). 
Nevertheless, the book demonstrates at the same time that the stock market is dangerous, because one can be captured by optimism and end up hit financially. Contrary to Cardoso (2006), Petram (2011, p. 211) concludes that De la Vega's book was meant to warn and to entertain.

A difference between stocks and other financial instruments in the seventeenth century, is that the valuation of stocks is not as developed as the valuation of life annuities (Poitras, 1996, p. 22). Instead, the attention around stocks is directed to manipulation and bubbles, as illustrated by Van Dillen (1935), De la Vega (1688), and Kindleberger (1978).

\section{3: Efficient Market Theorists in the $19^{\text {th }}$ and Early $20^{\text {th }}$ Century}

\section{Jules Regnault and Henri Lefèvre}

In France of the 1860s, the theory of behavior of financial markets takes the leap from manipulation and bubbles to the efficient market theory. The development of the theory occurs against the background of the emerging stock market of Paris in the $19^{\text {th }}$ century: the stock market expands from 3 listed stocks in 1800 to more than 1000 stocks in 1900 (Jovanovic, 2006, p. 170). Arguably, there was a need for analysis in the form of graphical presentations, statistics, probability theory, and financial-economic theory (ibid., p. 170). At that time, popular investment literature was widely available:

The 'science of financial investments' [popular investment literature in the second half of the eighteenth century in Europe and the United States] was meant, among other things, to disentangle investment from gambling. [....] The most important instruments were financial charts, price tables and balance sheet analyses (Preda, 2006, p. 152, my insertion).

In the $19^{\text {th }}$ century, investing in stocks is associated with gambling, but how could investing in stocks be legitimized (Preda, 2006, p. 151)? The legitimization of 
investing in stocks is done by vernacular science. The vernacular science of financial investments attempts to dispose of the unethical connotation of investing by turning investing into a scientific object:

'Vernacular economics' is understood to comprise heterogeneous sets of practices, know-how techniques and rationalization procedures with the help of which social actors make sense of their economic environment and of the economic consequences of their own actions. [...] vernacular economics mixes tacit, commonly shared assumptions and knowledge about economic processes with non-systematic rationalizations (Preda, 2006, p. 150).

So how does vernacular finance relate to the then emerging efficient market theory? Vernacular science draws the analogy of financial markets with physics and engineering, and adopts the idea of rational behaviour as the guideline for stock holders. Rational behaviour in the form of self-control and the study of information, is promoted as the key to successful investing:

They [The how-to brochures of vernacular finance] aimed to convince their readers that investments were not only lucrative, relatively sure, but also legitimate sources of income, given that a few rules were respected. These rules, incessantly repeated, concerned individual behaviour, on which financial success was made dependent. Lack of emotions, capacity of self-control, continuous study of the markets, and monitoring of the joint-stock companies were made into fundamental conditions of successful investments (Preda, 2006, p. 153, my insertion).

A part of vernacular finance results in the efficient market theory, because vernacular science includes probabilistic and abstract reasoning. Within the history of finance, Louis Bachelier (1870-1946) is generally considered to be the first efficient markets theorist. Efficient market theory is, in short, the theory that prices at the financial markets behave randomly. Yet, his fellow Frenchmen Jules Regnault (1834-1894) and Henri Lefèvre (1827-1885) precede Bachelier in inventing similar theories about the financial markets. 
Regnault (1863) writes about the random walk hypothesis of stocks in Calcul des chances et philosophie de la Bourse (Preda, 2006, p. 149). He invents a stochastical theory of financial markets. Bachelier's work (1900) s based on Regnault, especially the random walk hypothesis (Jovanovic, 2006, p. 191). Jovanovic considers Calcul des chances et philosophie de la Bourse a seminal contribution to finance. Regnault's work is unique in a period in which even economics is merely becoming a part of the university institutions (ibid., p. 211).

To illustrate the claim of Regnault's importance: he invents the square root formula of risk and time, analogous to the diffusion theory of heat and gases:

\footnotetext{
Regnault also produced the earliest financial theory of the dispersion of securities prices over time. He argued that while the mean value of a financial instrument may converge to some expected value, it was seemingly buffeted up or down by myriad external and subtle forces (Read, 2013, p. 19).
}

In 1870, Lefèvre invents the graphical presentation of the pay-off of an option, which Bachelier uses and which is still used today, and thinks up an economic theory about the place of financial markets in the circulation of goods (Jovanovic, 2006, pp. 169-171). For Lefèvre, the stock market can contribute to a better society (Preda, 2006, p. 152).

\section{Louis Bachelier}

Bachelier (1900) could be acknowledged as the father of modern finance and should replace Markowitz, who is usually given the credit (Read, 2013, p. 1). Bachelier anticipates the Black \& Scholes option formula and formulates the random walk of stock prices. He proposes to view the financial market as a phenomenon which can be expressed by scientific statistical laws, and presents an alternative for the financial markets as mere gambling on price changes (ibid., $\mathrm{p}$. 28). At the same time, his claims about the predictive capability of statistics are modest and nuanced. Interesting to note is that his $\mathrm{PhD}$-supervisor Henri Poincaré (1854-1912), a mathematician and philosopher of science among other things, did 
not favour probabilistic modelling of dynamic systems, but nevertheless lets Bachelier succeed (Zimmerman \& Hafner, 2006, p. 254).

Bachelier's work is not recognized in his time. It took 60 years before his seminal work starts to get noticed (Bernstein, 1992). The ideas of Bachelier (1900) will be presented here by a number of citations. Bachelier discerns two sorts of causes, the first being 'events' which influence the price of securities, and the second that the stock market is reflexive:

The influences which determine the movements of the Stock Exchange are innumerable. Events past, present or even anticipated, often showing no apparent connection with its fluctuations, yet have repercussions on its course.

Beside fluctuations from, as it were, natural causes, artificial causes are also involved. The Stock Exchange acts upon itself and its current movement is a function not only of earlier fluctuations, but also of the present market position (Bachelier, 2011/1900, p. 1).

So many causes exist, that a forecast, a point estimate, is not possible. Moreover, probability theory is not applicable, meaning that the fluctuations of the stock market do not resemble the outcomes of the tossing of a coin:

The determination of these fluctuations is subject to an infinite number of factors: it is therefore impossible to expect a mathematically exact forecast. Contradictory opinions in regard to these fluctuations are so divided that at the same instant buyers believe the market is rising and sellers that it is falling.

Undoubtedly, the Theory of Probability will never be applicable to the movements of quoted prices and the dynamics of the Stock Exchange will never be an exact science (ibid., p. 1).

Bachelier is evidently aware that probability theory for investing does not stochastically predict. His use of probabilities falls in the category of Knightian uncertainty, not Knigthian risk (Read, 2013, pp. 30-31). Probability theory has a different purpose in Bachelier's theory: probability theory can be an instrument to capture momentary insight or opinion about the level of fluctuations on the stock market: 
However, it is possible to study mathematically the static state of the market at a given instant, that is to say, to establish the probability law for the price fluctuations that the market admits at this instant. Indeed, while the market does not foresee fluctuations, it considers which of them are more or less probable, and this probability can be evaluated mathematically (Bachelier, 2011/1900, p. 1).

Though Bachelier was ahead of his times, he continues on the ideas of Regnault and Lefèvre (Poitras, 2006, p. 5). He does not mention Regnault of Lefèvre when he presents his formula:

Up to the present day, no investigation into a formula for such an expression appears to have been published: that will be the object of this work (Bachelier, 2011/1900, p. 1).

Bachelier does not mention information as the driver of changes in the prices on the stock market, but refers to 'innumerable factors' or causes. He seems to include more causes then Fama (1965a, 1965b, 1970), who reduces all causes to information associated with news about the external world, which should shed a light on the fundamental value of the securities traded on the market. Bachelier explicates his doubt about the transfer mechanism of influences or 'information' into the valuation of stocks. Yet, Read (2013) seems to view 'reflection of information' and 'all sort of causes' as similar:

Yet, Bachelier was asserting that prices should be modelled as without memory, reflected all available information, and buffeted by random forces of various strength, which he would go on and describe as characterized by a Gaussian distribution (Read, 2013, pp. 27-28).

Read (2013, p. 29) argues that a random walk presupposes informational efficiency. If information would only be available to some, the stock market would not be a random walk anymore, because the market would be predictable for some of the market participants. Read explains the efficient market hypothesis as a lack of foresight about the future, and assumes that without future changes the value of a security can be estimated correctly, though maybe merely as a convention: 
All market participants have equal knowledge of past events, and the current prices each presumably exhibit an identical lack of foresight of future events, hence the observed price is assumed to be correct, if only perhaps as a matter of financial philosophy or faith (Read, 2013, p. 29).

Bachelier believes that buyers and sellers on average do not know more about the future, and claims that the information would already have been included in the price if it would have been available (Bernstein, 1992, p. 20). For Bachelier it means that the expected return of the speculator is zero. Otherwise stated: investing is a fair game because the chances of winning and losing are equal.

Bachelier's work is characterised by originality, both in the theory of financial economics and the modelling of the theory in which he discovered the formula for the random walk of stocks comparable to the so-called Brownian motion:

Beyond its pioneering treatment of option pricing, efficient financial markets and rational expectations, Bachelier's dissertation provided a mathematical formalization of the stochastic process later identified with the Brownian motion of molecules [...] (Dimand \& Ben-El-Mechaieckh, 2006, p. 225).

Though actuaries have used the notion of probability to perform calculations in life insurance, the techniques were not related to financial markets, or options. He is the first to develop a sophisticated option formula which resembles like the BlackScholes options formula (Read, 2013, p. 26). Volatility as a measure of risk is a part of Bachelier's approach to option pricing and is not invented by Markowitz (ibid., p. 32). Bachelier's work is even compared to Einstein's work:

Five years later, Albert Einstein (1879-1955) used the same reasoning in physics and went a step further by calibrating the model and the resulting diffusion rate to determine the size of atoms (Read, 2013, p. 25).

Bachelier's dissertation of 1900 attracts attention after some fifty years by Savage (Dimand \& Ben-El-Mechaieckh, 2006, p. 233). Savage informs others such as Paul Samuelson of his discovery. But Savage's discovery of Bachelier is not a 
total coincidence, because Savage was aware that possibly interesting probability theorists had existed in France:

Savage was then browsing in the writings of early twentieth-century French probability theorists and was receptive to lost treasures [...] (Dimand \& Ben-ElMechaieckh, 2006, p. 233).

Read proposes two explanations why Bachelier's work, and that of Bruno de Finetti, remained unnoticed for such a long time: because of the 'balkanization' of science and the advanced level of their work:

Caught squarely in this unfortunate tendency toward the nationalization of scholarship was the work of Louis Bachelier in the early twentieth century, and, for that matter, the great mind Bruno de Finetti (1906-1985) a little later. The work of both of these brilliant scholars remained abstruse both for reasons of language and for the advanced nature of their mathematical and philosophical discoveries in the field of probability (Read, 2013, p. 16).

\section{4: Finance in the First Half of the $20^{\text {th }}$ Century}

\section{Irving Fisher}

After treating Bachelier and his French predecessors, who introduced the notion of the random walk and option pricing, we continue with a number of theorists on other issues in investment theory in the United States and the United Kingdom, such as Irving Fisher, John Maynard Keynes, and John Burr Williams. The American economist Irving Fisher (1867-1947) is well known for his unfortunate prediction in October 1929 before the crash that "stock prices have reached what looks like a permanent high plateau (Dimand, 2007, p. 45)." Nevertheless, Fisher is highly regarded as an economist because of his lasting contributions in for example monetary economics, illustrated by his famous equation of exchange of $M V=$ $P T$, his distinction between real and nominal interest rates, and his theory of debt deflation, in which the burst of the bubble is followed by a debt-fuelled depression and deflation (Tobin, 2008). 
With regard to financial economics, Fisher acknowledges a serious role for stocks as an investment, invents a portfolio approach, and systematizes the net present value method for investments. He stresses that the perception that bonds are a safe investment when the default risk is absent, is wrong, because bonds have purchasing power risk because of inflation (Dimand, 2007, p. 46). Yet, stocks have the inherent capacity to rise with inflation because companies can adjust their selling prices; stocks and bonds are subject to different risks, but on the whole stocks can be expected to have a better return in an inflationary environment (ibid., p. 46).

Fisher is ahead of his time by advocating the diversification of a stock portfolio and constructs for the purpose an investment theory with an expected return (the dividend yield), risk (the standard deviation), and risk attitude (the coefficient of caution) (ibid., p. 48). Preceding Markowitz (1952), he advocates the use of probability distributions for making investment decisions.

Though the method of net present value has existed for a long time, it is Fisher who seems to have proposed that it is appropriate for any investment decision, both for a firm and an investor on the financial market (Rubinstein, 2006a, p. 7). His idea is continued by the dividend discount model of Williams (1938) for the valuation of stocks (Dimand, 2007, p. 49).

\section{John Maynard Keynes}

Keynes (1883-1946) formulates a theory of investing under uncertainty which combines the psychology, reasoning, and consequences of human interacting. First, he illustrates the functioning of a financial market by the metaphor of a beauty contest. The beauty contest held in a certain newspaper at that time compares with a financial market under uncertainty in which its participants behave reflexively on each other (Keynes, 1997/1936, p. 156). To win the beauty contest, the participants have to choose the six most popular faces out of a hundred 
pictures. To win, a participant has to predict which face the other contesters will choose. So, if the stock market resembles the beauty contest, stock prices are not about some fundamental value, but about the perceived ideas of other market participants and their interaction.

The lack of knowledge about the future implies for Keynes that the current market valuation is not correct in the sense that the valuation of, say, a stock properly discounts the future. The current price of a stock cannot be correct, unless by coincidence, because we lack the knowledge to calculate the correct price. Keynes summarizes with three principles (the present is the best guide for the future, the current prices reflect the available information, the 'market' knows more than the individual) how to act under uncertainty and then formulates an efficient market theory avant la lettre, in which the consequence of uncertainty is that financial markets are instable. Keynes's three principles and his efficient market theory will be quoted at length because it is a predecessor and competitor of the modern efficient market theory, with the fundamental difference that his theory does not assume predictability like the CAPM does::

(1) We assume that the present is a much more serviceable guide to the future than a candid examination of past experience would show it to have been hitherto. [....].

(2) We assume that the existing state of opinion as expressed in prices and the character of existing output is based on a correct summing up of future prospects, so that we can accept it as such unless and until something new and relevant comes into the picture.

(3) Knowing that our individual judgment is worthless, we endeavour to fall back on the judgment of the rest of the world which is perhaps better informed. [....].

Now a practical theory of the future based on these three principles has certain marked characteristics. In particular, being based on so flimsy a foundation, it is subject to sudden and violent changes. The practice of calmness and immobility, of certainty and security, suddenly breaks down. New fears and hopes will, without warning, take charge of human conduct. The forces of disillusion may 
suddenly impose a new conventional basis of valuation. [...]. At all times the vague panic fears and equally vague and unreasoned hopes are not really lulled, and lie but a little way below the surface (Keynes, 1978/1937, pp. 114-115, his italics).

Moreover, besides reflexivity, irrational optimism of investors, that is their animal spirits, overshadows the importance of probability calculations:

Most, probably, of our decisions to do something positive, the full consequences of which will be drawn out over many days to come, can only be taken as a result of animal spirits - of a spontaneous urge to action rather than inaction, and not as the outcome of a weighted average of quantitative benefits multiplied by quantitative probabilities (Keynes, 1997/1936, p. 161).

For Keynes, decision making is about animal spirits, which in combination with uncertainty and interaction of market participants, characterize financial markets. Interestingly enough, Keynes anticipates three arguments against neoclassical economics: decision makers are irrational, information about the future is to a great extent uncertain, meaning unknowable, and markets behave reflexive as witnessed by the beauty contest and his version of the 'efficient market theory'.

\section{Benjamin Graham and John Burr Williams}

Another perspective on the theme of predictability is the idea that the value of a security is not same as its price, and that the value of a security can be discovered. The notion of predictability stems from the conviction that price and value converge in the end (Bernstein, 2005/1992, p. 117). If the current value of a security is lower than its market price, valuation theory indicates a buying opportunity, a prediction that the price of the stock will rise in the future. Valuation is a cornerstone of old and modern finance, and at the same time ambiguous: is the value of a stock its book value (which can theoretically be sold on other markets), the net present value of its future cash flows or dividends (or the lowest of the two), or a product of supply and demand on the financial market (Bernstein, 2005/1992, p. 118)? 
Benjamin Graham (1894-1976) uses security analysis to gain insight in the value of a stock. His theory proposes a defensive way of investing and is based on rules of thumb. Graham et al (1934) use past data to estimate the value of a company, for example its book value. The investment theory of John Burr Williams (1900-1989) values an individual security by looking at its future dividend. The valuation theory of Williams (1938) assumes that the value of a security equals the net present value of the future dividends of the security. In Williams's model, the interest rate is an appropriate part of the calculation. His approach combines theoretical and common sense: the value of a stock is the same as its future returns discounted, which makes his valuation theory theoretically superior to Graham's method. But Graham's method is more practical. Later portfolio theory assumes the theory of valuation as valid; the seminal work of Markowitz $(1952,1959)$ and Fama (1965a, 1965b) refers to the valuation method as the way to discover the intrinsic value of a stock.

Yet, the theories of valuation are problematic: "Even modern financial economics lacks a theoretical model of stock pricing with any practical accuracy [...] (Poitras, 1996, p. 22, his emphasis)." To start with Graham's method, his practical backward looking method of valuation has the problem of the interpretation of accounting data: what does the book value tell about the value of the stock? Concerning risk, Graham's approach incorporates risk in a practical way by investing in value stocks with a safety margin between the stock price and its supposed value. But his approach does not work if all stocks are expensive in a historical perspective, because all stocks will then have a high value. Anyway, the appropriate level of valuation, such as the ratio of book value to price, or price to earnings, is the key problem, for Keynes (1936) a reason to consider valuation as an instable convention. Graham's method merely analyses whether a valuation is high or low relative to its past, but what if other factors determine the level of valuation, say the central bank policy or a positive economic outlook? I do not 
mean to suggest that historical valuation measures such as the price-earnings ratio are useless, but valuations such as the historical price to earnings ratio can stretch enormously and can stay that way for a long time. So, the expectation that valuation predicts teleologically cannot be met: valuation merely predicts in a general fashion, but renders no specific prediction. If valuation would predict, than the return of stocks would become stochastically predictable, which is not possible as I will try to underpin by arguments of economists, and a thought experiment invented by myself in the next Chapter.

Considering Williams's forward looking valuation method, it has, of course, the difficulty of forecasting the future earnings or dividends of a stock. A special difficulty arises in the valuation of growth stocks if the growth factor is higher than the discount factor: in William's model the value will become infinite at a large horizon (Durant, 1957). But valuation has to incorporate risk as well, so it is not merely about the expected value, but also about the probability distribution of the value. Yet, how should the investor, who is likely to be risk averse, weigh the probability distribution of the future earnings? First, we would have to investigate whether the value of a security is subjective, meaning depending on the preferences of the investors, or objective, meaning that risk is neutrally valued. Utility theory informs us that preferences matter and that because of risk averseness, the subjective element should be in the valuation of a stock. Thus, if investors find themselves in a financial crisis they become more cautious and might weigh negative outcomes of the probability distribution of earnings higher than before, which means that the value of stocks should go down in a financial crisis, irrespective whether future earnings will be lower. Of course, the obvious solution to the problem of subjectivity would be to use the risk neutral valuation method like in option theory. Yet, risk neutral pricing of options is grounded in the possibility that an option can be hedged by a position in stocks or by the put-call parity, a possibility which is absent for stocks. And, the pricing of an option has only one dimension, because the buyer and the seller of an option only have to 
agree about the future volatility of a stock, since an option price does not incorporate an expected value above the risk-free return. But stocks are assumed to have a return that differs from the risk-free return, which has to be distilled from its future earnings or dividends. To conclude, for the valuation of a stock three estimates have to be made: its expected value, the probability distribution of the value, and the level of risk averseness to weigh the probability distribution. Though modern investment theory, as will be shown later on in the Chapter, proposes a solution for determining the risk and return of stock in a portfolio context, modern investment theory nor valuation theory predicts stochastically, for which I will give a number of arguments in the next Chapter of the dissertation.

\section{Empirical Work on Predictability and the Random Walk}

Predicting financial markets is hard, which both theory and empirical evidence illustrate (Bernstein, 1992). In the first half of the $20^{\text {th }}$ century, Alfred Cowles, Holbrook Working, and Frederick Macaulay, investigate the ability to predict financial markets. Alfred Cowles (1891-1984) founded the 'Cowles Commission for Research in Economics' in January 1932 to promote research into financial markets (Bernstein, 2005/1992, p. 33). Cowles (1933) investigates the results of the advice of investment analysts for beating the market average and predicting the stock market. The outcome of his investigation denies that the advice of investment analysts outperforms the market index or predicts the market as a whole. Cowles wonders about the economic logic of offering advice about the outcome on financial markets for a fee, because exploiting the predictions, if correct, would result in a higher income than the fee provides:

\footnotetext{
Market advice for a fee is a paradox. Anybody who really knew just wouldn't share his knowledge (Bloom, M.T. (1974), Rogues to Riches, Warner Books, New York, in Bernstein, 2005/1992, p. 35).
}

Another study of Cowles (1944) about the prediction of the stock market again concludes that investment advice for predicting the stock market makes no sense 
(Bernstein, 2005/1992, p. 36). But it turns out that investors and practitioners are deaf to investigations such as performed by Cowles, and stick to their habits of continuing to believe in forecasting (ibid., p. 37). Cowles suggests that investors are in psychological need of believing that experts possess knowledge about the future course of the stock market:

Even if I did my negative surveys every five years, or others continued them when I'm gone, it wouldn't matter. People are still going to subscribe to these services [about the predictions of investment advisors]. They want to belief that somebody really knows. A world in which nobody really knows can be frightening (Bloom, M.T. (1974), Rogues to Riches, Warner Books, New York, in Bernstein, 2005/1992, p. 38, my insertion).

The public is not interested in Cowles's studies of unpredictability, but neither are academia at that time. There seem to be two reasons for the lack of interest in academia in his research:

1. economists did not regard financial markets as a serious topic at that time, 2. the majority of economists were not yet educated in mathematics or statistics in those days (Bernstein, 2005/1992, p. 93).

Besides Cowles, Holbrook Working and Frederick Macaulay are prominent investigators of the predictability of financial markets. Working (1934) studies wheat prices and concludes that the price changes largely follow a random walk by comparing wheat prices with random outcomes. Macaulay (1938) refines the idea of random outcomes to a more complex level of randomness, and compares the stock market to "a loaded pair of dice, with the load shifted from time to time (Fox, 2009 , p. 27)". The reason for Macaulay for denying the normal distribution as a valid assumption, is that people take decisions about the future by which they react on the decisions of others, which is not reflected in a normal distribution: 
Of course, the disturbing effects that such factors as presence of emotion, lack of logic and insufficiency of knowledge have on the economic behavior of individuals would not merit the attention we are giving them if socially they always 'cancelled out'. If the vagaries of individual conduct were always 'normally' distributed round a strictly rational 'mode', in other words, if the 'deviations' were of the nature of 'accidental' rather than, for example, 'systematic' or 'constant' errors, their curbing effects on the development of economics as a strictly logical social science might be small or negligible $[\ldots]$. [...]. It [the normal distribution] is and always will be thoroughly unreal (Macaulay, 1938, pp. 11-12, his italics, my insertion).

In the first half of the 20th century, the pioneering research of Cowles, Working, and Macaulay, results in an empirical underpinning that investment advice does not predict. Macaulay (1938) takes the analysis of returns on financial markets a step further by denying that financial markets display the type of randomness that can be captured by the normal distribution; an outcome which has not been adopted in the later modern investment theory.

\section{Chartist Theory}

To complete the highlights of the development of finance theory in the first half of the 20th century, chartist theory, or technical analysis, is treated briefly now. The central idea of chartist theory is that past data of the stock market reveal everything an investor needs to know about the market. The Dow Theory is worth mentioning because it is an example of a chartist theory, which together with the theory of valuation still belongs to the current practice of investing. The Dow Theory is a theory named after the work of Charles Dow (1851-1902) for predicting stock prices (Bernstein, 2005/1992, p. 24). Dow's name is familiar because he invented the well-known Dow Jones Average. Underlying Dow's theory is that trends tend to maintain until the market sends a signal for its reversal. Dow uses the metaphor of ebb and flood. The expression often used in practice of a 'correction' of the market is a term from Dow's theory. He actually never uses the term 'Dow Theory' himself (ibid., p. 28). He is more interested in interpreting history than making a theory for predicting its future. 


\section{5: Markowitz's Investment Theory}

\section{Portfolio Theory}

Harry Markowitz's portfolio theory $(1952,1959)$ is conventionally regarded as the starting point of modern finance (Poitras, 2007, p. 4). With portfolio theory, an investor composes a statistically legitimate portfolio of individual securities, or an asset mix of bonds, stocks, and real estate (Markowitz, 1952, p. 91). He founds his theory on mathematical statistics: he assumes that '[...] 'beliefs' or projections about securities follow the same probability rules that random variables obey (Markowitz, 1999, p. 5)." The metaphor that investing is mathematical statistics is the modern aspect of investment theory. In short, the mathematics in Markowitz's article of 1952 illuminates that a higher return is accompanied by a higher risk and that diversification is a sensible idea:

It boils down to nothing more than a formal conformation of two old rules for investing: Nothing ventured, nothing gained. Don't put all your eggs in one basket (Bernstein, 2005/1992, p. 44).

In his Nobel Lecture, Markowitz summarizes his contribution to price theory by showing how a rational individual investor would invest:

My work on portfolio theory considers how an optimizing investor would behave, whereas the work by Sharpe and Lintner on the Capital Asset Pricing Model (CAPM for short) is concerned with economic equilibrium assuming all investors optimize in the particular manner I proposed (Markowitz, 1990, p. 279).

The price theory of consumers and producers differs from the price theory of capital markets: price theory relates price to quantity, while Markowitz (1959, p. 259) leaves out quantity. Instead, he relates the expected price gain, the investment return, to risk, because risk features in the future price of a security. Though he does not model quantity, the probability beliefs about investment returns could include the influence of quantity, because the probability beliefs can include any theory of economics. Please note that, because Markowitz adheres to the theory of 
personal probability, for him risk and uncertainty mean the same. Yet, to repeat, Knight (1921) distinguishes risk from uncertainty.

The portfolio approach of Markowitz changes the notion of investment risk. According to Bernstein (2005/1992, p. 54) “Markowitz's most original contribution" was to distinguish individual stock risk and portfolio risk. A portfolio risks less than an individual security, because a portfolio benefits from diversification as the ups and downs of its individual securities partly compensate. An efficient portfolio makes the most of diversification. Efficient means that a portfolio returns maximally at a particular level of expected risk, or to put it another way, risks minimally at a particular expected level of return. As the measure of risk, he proposes the standard deviation of investment returns. The standard deviation measures the dispersion around the expected return.

Portfolio theory specifies and maximizes the potential of diversification. Markowitz's reading of history is that no such theory of diversification then existed: "What was lacking prior to 1952 was an adequate theory of investment that covered the effects of diversification when risks are correlated [...] (Markowitz, 1999, p. 5, his italics)." John Burr Williams, applies in his seminal work The Theory of Investment Value (1938), the law of large numbers to discover the diversification potential of a portfolio. The law of large numbers implies that the returns of the securities involved behave stochastically independent: then, the risk of the portfolio disappears at a large number of securities in the portfolio. Yet, Markowitz becomes aware that Williams overestimates the potential of diversification: "The returns from securities are too intercorrelated. Diversification cannot eliminate all variance (Markowitz, 1952, p. 79)." The risk that can be eliminated, stems from idiosyncratic risk, that is the specific risk of securities. But systematic, or market risk, cannot be diversified. The notion of market risk will explained further on in the current Chapter. In the Appendix A of the current Chapter, the mathematics of diversification is explained. 
Mathematical statistics supplies portfolio theory with ideas for efficiency criteria and computing procedures. The efficiency criteria in portfolio theory focus on what is important to an individual investor:

[...] determine the type of conclusions sought. [...]. The criteria of an analysis is its guide to what is important and unimportant, relevant and irrelevant (Markowitz, 1991/1959, p. 205).

The criteria of risk and return select the efficient portfolios, and are mirrored in the investor preferences in the utility function. Markowitz proposes for individual investors a quadratic utility function (ibid., p. 286 and further), here in a simplified form:

Utility $=$ expected return - risk aversion $\cdot(\text { expected return })^{2}$

The utility function treats the expected return as positive, and the (expected return $)^{2}$, the standard deviation, or expected value of the squared returns, as negative. Markowitz elaborates his choice of utility function and efficiency criteria in his seminal work of 1959 . He chooses to reduce the probability distribution to the criteria of mean and standard deviation, because the standard deviation is familiar in statistics, and has computational ease.

The beliefs about expected returns, risks, and correlations, the subject of my dissertation, are, together with the efficiency criteria, put in Markowitz's optimisation procedure to calculate the efficient portfolios. The efficient portfolios, or efficient set, contains, to repeat, portfolios with the highest return at a particular risk. An investor chooses the portfolio which maximizes her utility. In Figure 2.1 the indifference curves, curves with combinations of risk and return at which the investor is indifferent, approach from the top left to meet the efficient 
set, which approaches from the bottom right: the indifference curves of the investor and the efficient set meet at the point of tangency.

Figure 2.1: Utility maximization in capital markets according to Markowitz

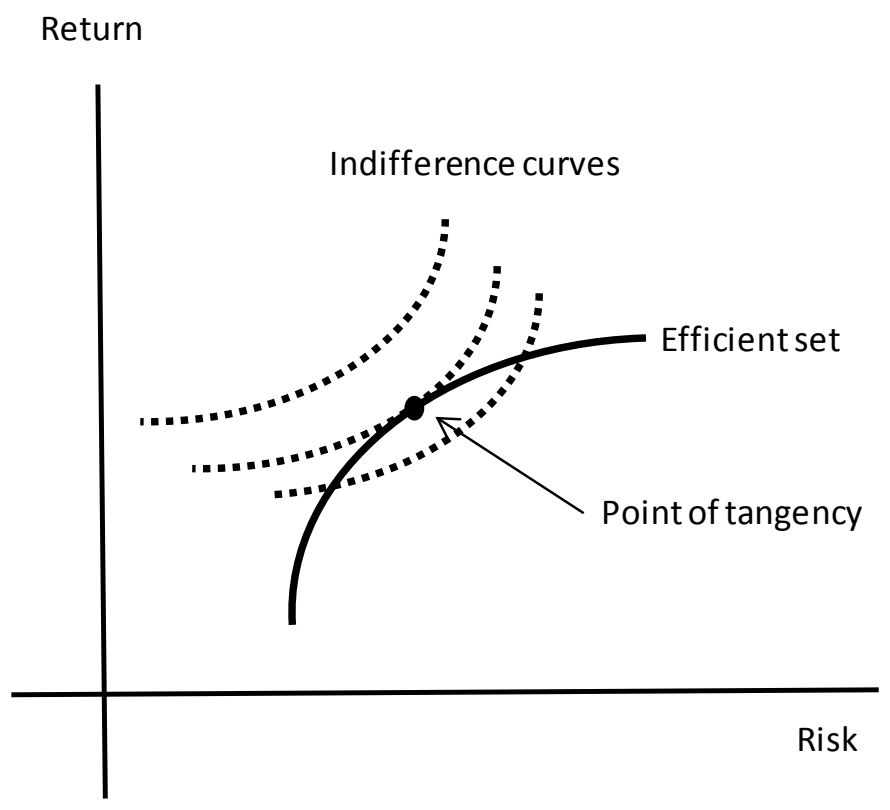

\section{Evaluation of Markowitz's contribution}

In the evaluation of Markowitz's theory, I will review the impact of diversification on risk, the use of the standard deviation as the measure of risk, Markowitz's ideas about his possible predecessors, the role of Markowitz's theory for further theory development, and the practical use of Markowitz's approach.

Markowitz finds a way to calculate the most interesting portfolios with respect to return and risk by optimising the benefits of diversification. But, "How big is big?" in diversification? "How big is big?" is a question of relevance about a theory or an empirical finding that McCloskey proposes to ask (McCloskey, 2000, 
p. 258). Markowitz has noted, to repeat, that a correlation of zero between securities is too optimistic. Let us perform a simple test of the contribution of diversification to a lower risk. The risk, the standard deviation, of a worldwide portfolio of equities is $17.7 \%$ annually measured during 1900 to 2011 : the risk of $17.7 \%$ includes the diversification effect of a huge amount of stocks (data from Dimson et al, 2012). Indeed, again, investing in stocks does not mean that the correlation coefficient between individual stocks is zero, or even close to it, otherwise the risk of stocks should become close to zero as historically measured by the standard deviation of stocks during 1900 to 2011 . Thus, within an asset class, diversification benefits to a certain extent by elimination of the idiosyncratic risk of individual securities, but a large part remains undiversifiable as illustrated by the level of the standard deviation of a large amount of stocks of $17.7 \%$.

A correlation of higher than zero means that investment returns of individual stocks are not independently distributed in contrast to the outcomes of throwing a dice. We will see in a next paragraph that for example Sharpe (1963) invents a common cause, bèta, to determine which part of the return of a security is caused by the stock market as a whole. Of course, stocks are not the only asset category, and the asset mix can diversify by mixing different asset categories, which could diversify. The diversification over diverse asset classes can be rewarding if a low correlation is accompanied with benign risk and return features: yet, in practice such categories are difficult to find.

Besides diversification by adding more securities, time could diversify investment returns as well. One can make the tossing of a coin amount to $50 \%$ heads and $50 \%$ tails, by tossing many coins at the same time, or, by tossing the same coin many times. Both ways result in the same probability distribution of approximately $50 \%$ heads and $50 \%$ tails, if the number of trials is large. Yet, time diversification in investing is not analogous to the tossing of coins. When tossing a coin, the basis of the calculation expands proportionally. But the case of coins is different from 
investing: at investing, the basis of invested capital is fixed at the starting point and merely diminishes or increases because of the returns obtained. Thus, the standard deviation around the mean return is a wrong measure for time diversification, because an investment portfolio is a capital at risk which is multiplied by returns. Therefore, risk, as measured around the value of invested capital, increases during time; think about the risk of multiple years of negative stock market returns. Samuelson (1969a) Merton (1973a), and Bodie (1995), demonstrate that time diversification does not exist in investing. Another way to illustrate the denial of time diversification is the relation of risk and time in option pricing as expressed by the so-called square root of time formula in which risk enlarges with the square root of time; not many people will deny that an option with a longer horizon is more expensive than the same option with a shorter horizon. Because it would be out of scope, I will not go into the debate on time diversification which offers other arguments, such as human capital, for the benefits of a longer investment horizon: the interested reader is referred to a summary of the subject by Mark Kritzman (pp. 50-57, in Bernstein \& Damodaran, 1998), or Pistorius (2004).

Markowitz (1959) chooses to reduce the probability distribution of investment returns to the criteria of mean and standard deviation. I have compared Markowitz's choice of efficiency criteria with alternatives, such as stochastical dominance, the inclusion of skewness, and criteria which focus on downside risk (Pistorius, 1991). Among other things, my conclusion was that stochastical dominance serves as a better efficiency criterion when probability returns are not normally distributed. The inclusion of skewness as a third criterion next to the mean and the standard deviation, is superior as well when probability returns are not normally distributed. Markowitz (1959) himself favoured the use of the semideviation, measuring only the downside deviation instead of the two-way measurement of the standard deviation. Nevertheless, Markowitz's choice for the standard deviation as the measure of risk seems defensible, because the standard 
deviation is familiar in statistics, and has computational ease in calculating efficient portfolios.

What is Markowitz's place in the history of finance? To answer the question, we will discuss the competing theories of Arthur Roy (1952) and Bruno de Finetti (1940), who seem to be the most important competitors of Markowitz. Other candidates could have been Fisher, John Hicks, and Dickson Leavens, who touched the subject of risk in investing, though not as systematically as Markowitz did (Bernstein, 2005/1992, p. 55). Roy (1952) seeks to explain the practice of diversification and proposes, instead of Markowitz's criterion of the standard deviation, a level of disaster as the main measure of risk, which probability has to be weighed against the expected return. Roy's criterion of 'level of disaster' anticipates the value-at-risk approach, which has later become popular in risk management. With the level of disaster as a risk criterion, he intends to get closer to the intuitions about risk as a situation one wants to avoid. According to Bernstein, Roy's article of 1952 reaches the same conclusions as Markowitz's article:

'Portfolio Selection' and 'Safety First' are similar in their lines of argument, and I am at a loss to explain why's Roy's paper failed to cut the swath that Markowitz's paper did (Bernstein, 2005/1992, p. 55).

Bernstein's best guess why Roy is forgotten is because he was the second to publicize (2005/1992, p. 56). In an article about the history of investment theory Markowitz (1999, p. 6) is willing to share the fatherhood of modern portfolio theory with Arthur Roy. Roy himself (1961) is critical about Markowitz's monograph on portfolio theory (1959) on a number of points, of which his criticism on how to obtain probability beliefs, is a subject which will be thoroughly analysed in the next Chapter of the dissertation: 
Before probability concepts can help us with the selection of our investments, we must be able to translate our expectations about future yields and process into subjective joint probability distributions. While Dr. Markowitz emphasises that past experience is unlikely to be a very good guide to future performance, he gives no clear indication of how either we, or our investment advisers, can provide ourselves with sufficiently precise or generally agreed expectations to merit their processing in an elaborate way (Roy, 1961, p. 99).

Roy and Markowitz published in the same period, but Bruno de Finetti (1940) made an earlier contribution along the same lines as Markowitz (Rubinstein, 2006b). So, another claim to the fatherhood of modern portfolio theory can be given to Bruno de Finetti (1940):

De Finetti's line of analysis bears a remarkable resemblance to what Markowitz would develop completely on his own twelve years later, and for which Markowitz won the Nobel Prize (Bernstein, 2007, p. 108).

It is not mere speculation that Finetti's theory could have been known before Markowitz's theory, despite being written in Italian:

He [De Finetti] was sufficiently well-known, however, to have been invited to the United States in 1950 by the eminent American mathematician, Leonard Savage, to deliver a paper on probability at a conference at Berkeley (Bernstein, 2007, p. 109).

In the investment practice of today, Markowitz's 'mean variance'-approach is used frequently to specify diversification and to underpin an investment strategy on the subject of diversification. But, hardly anyone uses the optimization procedure that Markowitz proposes, as his algorithm for calculating efficient portfolios has been replaced by more efficient, simpler, ways of calculation. One simplification stems from James Tobin (1918-2002), who finds a weakness in Markowitz's theory, that is that the riskless asset is absent, such as a treasury bill (Bernstein, 2005/1992, p. 71). Adding the risk-less asset to the analysis, leads to Tobin's (1958) so-called separation theorem: the selection of a Markowitz's efficient portfolio is separate 
from the decision to divide the portfolio in riskless cash and the risky portfolio (ibid., p. 72). The risky portfolio turns out to be the same in each linear combination of cash and risky assets (ibid., p. 73). Tobin's innovation simplifies portfolio theory to calculating the risky portfolio, but cannot make the calculations of Markowitz's risky portfolio easier.

Markowitz's optimization rule is difficult to execute in practice because the calculation procedure is complicated, and demands a lot of estimates of each individual security (Bernstein, 2005/1992, p. 57). For the practical application of portfolio theory, Markowitz himself made a suggestion in his monograph (1959). Because the returns of most stocks are correlated, all that had to be done was to consider whether each stock was more or less volatile than the stock market, and whether other influences were of relevance for a stock (Bernstein, 2005/1992, p. 77). Sharpe (1963) dealt with the problem of the application of Markowitz's portfolio theory. He calls his model the diagonal model, though in general it is called the single-index model (Bernstein, 2005/1992, p. 80). The diagonal model relates the risk of stocks to that of the market. Sharpe's tool works well as an approximation of Markowitz's model (ibid., p. 82).

\section{6: Efficient Market Theory}

\section{Paul Samuelson}

The empirical findings of the first half of the $20^{\text {th }}$ century, implying that the stock and commodities markets resemble a random walk, are continued in the second half of the $20^{\text {th }}$ century. Maurice Kendall (1953) analyses stock data and confirms Working's earlier findings for the stock market. Later in the fifties, Harry Roberts (1959) investigates technical analysis and suggests that all the typical patterns of technical analysis can be produced by chance as well (Bernstein, 2005/1992, p. 101). Michael Jensen (1968) corrects the relative performance for risk, because higher returns can also stem from taking more risk, and denies the outperformance 
of mutual funds (Bernstein, 2005/1992, pp. 140-141). Many later studies (see for example: Shefrin, 1999) deny the idea that the advice of investment analysts is profitable for investors.

The efficient market theory tries to explain the results of the empirical studies about the impossibility of beating the market index. One of the founding fathers of the efficient market theory is Paul Samuelson (1915-2009). Samuelson is a prominent economist, a Nobel Prize winner in 1970, and also contributed to the theory of financial markets. He clarifies that predictability of financial markets is not worthwhile to pursue as a way to make a living:

But a respect for evidence compels me to incline toward the hypothesis that most portfolio decision makers should go out of business - take up plumbing, teach Greek, or help produce the annual GDP by serving as corporate executives (Samuelson, 1974, p. 17).

According to Samuelson (1965b), the market price is the best estimate of its value. Indeed, if the intrinsic value of a stock would be higher and everyone would agree, then the stock price should go up. But, maybe some investors possess better information, for example because they have made a better analysis of the fundamentals of a security. Yet, looking at empirical studies about the performance of investment advice, or the performance of mutual funds, such a conclusion does not follow, on the contrary, the market performs better than a portfolio based on the investment advice or mutual funds. Samuelson stresses that information is relevant for financial markets, because information leads to changes in market prices (Bernstein, 2005/1992, p. 120).

His interest in informational efficiency came from his interest in option pricing (Read, 2013, p. 78). He seems the first to formulate the hypothesis of informational efficiency for financial markets: 
In his 'Proof that Properly Anticipated Prices Fluctuate Randomly', Samuelson put forth a simple proposition: in an informationally efficient market, prices must incorporate all information available to market participants. This implies that it must not be possible to forecast any additional price changes. Five years later, Eugene Fama more economically stated Samuelson's result by saying that 'prices fully reflect all available information' (Read, 2013, p. 77).

Non-predictability results from the economic law of full competition and is done in financial markets by arbitrage:

In the limit that perfectly arbitraged markets are frictionless and trading is costless, there is nothing that can be further anticipated in prices, and only randomness and unpredictability remain. Samuelson's innovation that arbitrage is a condition of equilibrium was of course not completely novel in finance (Read, 2013, p. 79).

Fama took Samuelson's ideas further into a comprehensive theory (Bernstein, 2005/1992, p. 126).

\section{Eugene Fama}

According to Fama (1970) the theory on efficient markets is not rigorous until the work of Mandelbrot (1963a, 1963b) and Samuelson (1965b). Though a lot of empirical work has been done in the securities and the commodities markets, the empirical work lacks, according to Fama, an economic theory. The research before Mandelbrot and Samuelson is based on the random walk as a fair game of probability with an expectation of zero, starting with Bachelier (1900):

Thus, though his contributions were ignored for sixty years, the first statement and test of the random walk model was that of Bachelier in 1900. But his

'fundamental principle' for the behavior of prices was that speculation should be a 'fair game'; in particular, the expected profits to the speculator should be zero.

With the benefit of the modern theory of stochastic processes, we know now that the process implied by this fundamental principle is a martingale (Fama, 1970, p. 389). 
The stochastic process to which Fama refers is a martingale process, which to his opinion is a better representation of the theory of the random walk. A martingale means:

A martingale is a mathematical model of a fair game, or of some other process that is incrementally random noise. The term, which also denotes part of a horse's harness or a ship's rigging, refers in addition to a gambling system in which every losing bet is doubled [...] (Karr, 2008).

Besides the technical betterment of the random walk by introducing the martingale, Fama develops the theory of the efficient market. His definition of an efficient market is "A market in which prices always 'fully reflect' available information [...] (Fama, 1970, p. 383)". The efficient market model does not expect the actual market behaviour to behave as an exact random walk, but the point for practice is whether a market participant can make profit by exploiting stock price patterns of the past (Fama, 1965a, p. 56).

Fama introduces as the cause of the randomness of price fluctuations new and reevaluated information:

The price changes in a speculative series can be regarded as a result of the influx of new information into the market and of the re-evaluation of existing information. At any point in time there will be many items of information available. Thus price changes between transactions will reflect the effects of many different bits of information (Fama, 1963, p. 425).

The definition of an efficient financial market is the same as for a market of goods in the theory of full competition, in which information is available to all participants at the same time:

Random walk theorists usually start from the premise that the major security exchanges are good examples of 'efficient' markets. An 'efficient' market is defined as a market where there are large numbers of rational, profit-maximizers actively competing, with each trying to predict future market values of individual 
securities, and where important current information is almost freely available to all participants (Fama, 1965a, p. 56).

Essential is that Fama believes in the possibility to value a security correctly by a fundamental analysis. In other words, he supposes that such a fundamental value exists. He says that the work of security analysts is worthwhile: otherwise the market would behave in another way. So, analysts make the market efficient. Because of uncertainty, however, the intrinsic value cannot be calculated exactly. Therefore the price will fluctuate randomly around the intrinsic value. Fama backs the idea that a stock has a fundamental value which can be analysed if one is willing to take the trouble, and which leads to the predictability of the price of a stock. I will now quote Fama at length on the topic of fundamental value and predictability because of its importance for the dissertation:

The assumption of the fundamental analysis approach is that at any point in time an individual security has an intrinsic value (or in the terms of the economist, an equilibrium price) which depends on the earning potential of the security. [....]. Through a careful study of these fundamental factors the analyst should, in principle, be able to determine whether the actual price of a security is above or below its intrinsic value. If actual prices tend to move toward intrinsic values, then attempting to determine the intrinsic value of a security is equivalent to making a prediction of its future price; and this is the essence of the predictive procedure implicit in fundamental analysis (Fama, 1965a, p. 55).

As a standard for rational behaviour in price theory, using fundamental analysis is for investors the right thing to do: it is sensible to find out new information about a security. But economists are also interested in the outcome if many analysts perform fundamental analysis:

That is, the existence of many sophisticated analysts helps make the market more efficient which in turn implies a market which conforms more closely to the random walk model. Although the returns to these sophisticated analysts may be quite high, they establish a market in which fundamental analysis is a fairly useless procedure both for the average analyst and the average investor (Fama, 1965a, p. 58). 
Surprisingly, the market is right, meaning that the price on the market is an accurate estimate of the intrinsic value of the security, because the estimates of security analysts are freely available:

In an efficient market, competition among the many intelligent participants leads to a situation where, at any point in time, actual prices of individual securities already reflect the effects of information based both on events that have already occurred and on events which, as of now, the market expects to take place in the future. In other words, in an efficient market at any point in time the actual price of a security will be a good estimate of its intrinsic value (Fama, 1965a, p. 56).

Fama (1970) distinguishes a few variations of market efficiency, strong, semistrong and weak, of which the semi-strong is, in his opinion, supported by empirical evidence:

He defined strong form efficiency as the most demanding information concept, in which all available public and private information has been incorporated and arbitraged into the price of a publicly traded security. [....].

More common is semi-strong market efficiency, in which market prices reflect only all publicly known information about the overall economy and the fundamentals of a particular asset, perhaps based on financial analyses and corporate news releases (Read, 2013, p. 105).

The intrinsic value changes because of new information, which will be priced in correctly. The process of the pricing in of new information is independent, which is enough for prices to be a random walk.

Fama (1965b) explains the random walk theory as consisting of two statistical hypotheses:

(1) Successive price changes are independent, and (2) the price changes conform to some probability distribution (Fama, 1965b, p. 35).

The first and most important hypothesis (ibid., p. 41) of independence means that price changes are not related in time, meaning that the price in a former period does 
not influence the current price, so one cannot predict on the basis of historical information. Being consequent, Fama rejects the predictability of chartist theories such as the Dow Theory because of independence of historical prices. He describes the chartist theories as:

Although there are many different chartist theories, they all make the same basic assumption. That is, they all assume that the past behavior of a security's price is rich in information concerning its future behavior. (Fama, 1965b, p. 34).

The second hypothesis of the random walk theory is that "the price changes conform to some probability distribution (ibid., p. 35)." The shape of the probability distribution does not need a specification for the theory to be valid, though the parameters of the distribution should be stationary (ibid., p. 41). Though, even stationarity is not necessary if independence is strictly met. Yet, for the investor the probability distribution matters because it determines the risk of the investment. The type of distribution is relevant for using statistical tools in empirical work as well. The normal distribution is an 'obvious' candidate for stock market returns, an assumption which is questioned by Benoit Mandelbrot (1963a, 1963b, 2004 with Hudson) who claims that distributions of financial markets have larger tails and peeks than assumed in the normal distribution:

Mandelbrot's main assertion is that, in the past, academic research has too readily neglected the implications of the leptokurtosis usually observed in empirical distributions of price changes (Fama, 1965b, p. 42).

Mandelbrot's theory will be explained at length later on in the Chapter. He also happens to be Fama's dissertation supervisor. Fama agrees to Mandelbrot's view that outliers in the data should not be easily ignored:

Unlike the statistician, however, the investor cannot ignore the possibility of large price changes before committing his funds [...] (ibid., p. 42). 
Fama (1963, pp. 426-427) acknowledges Mandelbrot's points that under the Paretian probability distribution:

- the variance as the measure of dispersion becomes meaningless

- if the variance is infinite, statistical tools for the normal distribution become unfit

- the measure of the central tendency becomes doubtful because one extra data

point can change the average

- investing is more risky

- stop loss orders do not work because of discontinuity of prices.

Fama (ibid., p. 428) confirms in his PhD-thesis that the data analysis of changes in stock prices show that the Paretian hypothesis that Mandelbrot advocates, is valid:

The conclusion of the dissertation is that for the important case of stock prices the stable Paretian hypothesis is more consistent with the data than the Gaussian hypothesis (Fama, 1965, p. 428).

Though later in his career, Fama does no longer follow Mandelbrot's theory and restricts himself to conventional statistics.

\section{Evaluation of the Efficient Market Theory}

In an evaluation of the efficient market theory, Fama (1965b, p. 36) distinguishes between two possible theories or views about financial markets which are consistent with independence of historical market prices. The first theory, a psychological one, asserts that the price mechanism on the financial markets has no relationship to the economic and political reality, and is primarily based on noise related to speculative behaviour of investors:

Independence of successive price changes for a given security may simply reflect a price mechanism which is totally unrelated to real-world economic and political events. That is, stock prices may be just the accumulation of many bits of randomly generated noise, where by noise in this case we mean psychological and 
other factors peculiar to different individuals which determine the types of 'bets' they are willing to place on different companies (Fama, 1965b, p. 36).

The second theory, which Fama advocates, of financial markets covering independence of historical market prices is based on economic and political analyses (Fama, 1965b, p. 36). By analysing, investors try to find out the intrinsic value of securities. An intrinsic value is consistent with the efficient market theory. An intrinsic value is non static, and changes because of new information. The intrinsic value of a security is not known for sure and will be estimated differently by participants, which creates noise.

Fama (1965b, p. 36) finds the psychological view unappealing, but what does he mean by that? That such a view is not realistic as an explanation because the behaviour assumed is irrational? His argument is that many people in the industry, analysts and fund managers do not agree as noticed by their behaviour. But that argument does not persuade, being that the argument is that something is true because it is common practice:

Even random walk theorists, however, would find such a view of the market unappealing. Although some people may be primarily motivated by whim, there are many individuals and institutions that seem to base their actions in the market on an evaluation (usually extremely painstaking) of economic and political circumstances. That is, there are many private investors and institutions who believe that individual securities have 'intrinsic values' which depend on economic and political factors that affect individual companies (Fama, 1965b, p. 36).

Fama presents the alternative for his theory of real intrinsic value of a security as an extreme one: for him the alternative theory is that the market is merely psychology. But eliminating psychology from markets is a rather reducing move, a move intended towards full rational behaviour. It seems plausible that both psychology and economic factors play a role in the stock market. For example, 
that there is some relation between the market and the external world over which a psychological overlay is placed, like in Keynes's beauty context.

A criticism from within mainstream economics on the efficient market hypotheses is invented by for example Lucas (1978). He claims that rational investors using all available information is a necessary, but not a sufficient condition, for a random walk:

An alternative view believes that these residuals will not be mere white noise even if prices have reflected all available information. For instance, Nobel laureate Robert Lucas (1978) and Stephen LeRoy (1973) created plausible scenarios in which prices fully incorporate all information but still behave unlike a random walk (Read, 2013, p. 84).

For example McCloskey \& Klamer (1995) claim that there is another fundamental problem with the efficient market theory: that is, what the available information is about. Does information lead to predictability and equilibrium, or is the flow of information merely loosely reflected in the market, because the transfer mechanism from information to valuation is ambiguous, because of the interpretation of information and the problems of valuation? Only simple information has straightforward implications for the value of a stock, say a takeover bid which is certain to take place, but most information has no clear implications. Fisher Black (1986) wrote about the phenomenon of noise to clarify its difference with information. Simple information is processed instantaneously because its effect on the value of the stock is clear. The other information or noise is priced in a subjective way, which is hard to predict or to arbitrage.

Besides the existence of a fundamental value, early writers such as Bachelier and Keynes, and the critics of the efficient market theory which will be treated later on in the Chapter, show that the reduction to information as the cause of randomness, ignores other causes, such as internal market dynamics, self-reflexivity, 
expectations, beauty contest dynamics in trying to outguess the others, bubbles, behavioural causes, and other psychological mechanisms.

\section{7: $C A P M$}

\section{Capital Asset Pricing Model}

There is a continuum of old and modern finance through security valuation. Fama agrees with Markowitz on the added value of security analysis, but Fama takes the activities of stock analysts one step further in the analysis of financial economics by examining the effect of competition; full competition in a goods market leads to absence of profits, full competition under security analysts leads to zero extra profits in the security markets. The subject of the current paragraph, the Capital Asset Pricing Model, aka the CAPM, incorporates Fama's and Markowitz's approach. After Markowitz determined how a rational investor would act, the next logical step was a formulation of the market equilibrium of expected risk and return. The CAPM is a product of the economic logic of diminishing marginal utility, meaning that risk is rewarded, and full competition, meaning that the market index cannot be beaten. According to Bernstein (1992) a number of people worked independently on the formulation of such a model: Jack Treynor (1962), John Lintner (1965), Jan Mossin (1966), and Sharpe (1964). I will focus on the contribution of William Sharpe (1964), who is probably the most well-known founder of the CAPM.

In the CAPM, an optimal portfolio is always at the capital market line, which combines the risk free return and the market portfolio. The capital market line in Figure 2.2, combines the risk free return and the market portfolio, the so called two fund theorem, which results in a higher level of efficiency than the efficient set of Markowitz. The market portfolio consists of all securities, which are assumed to be correctly priced, so no advantage could be gained by choosing another portfolio than the market portfolio. Because risk diminishes automatically by putting 
securities in a portfolio, only undiversifiable risk yields an extra return above the risk-free rate. Thus, unsystematic or idiosyncratic risk, the risk belonging to a specific stock which is independent of the market, does not matter for the value of the stock because it will be diversified away. For a stock with a large unsystematic risk, i.e. with diversification potential, it means it should have a higher value than a fundamental analysis would result to in isolation. Indeed, the stock should have a return which relates to merely the market risk. For fundamental analysis this means the CAPM becomes a fundamental part in judging the value of a stock, not only the fundamentals matter, but also the interplay with the risk of the market.

\section{Figure 2.2: Equilibrium in capital markets}

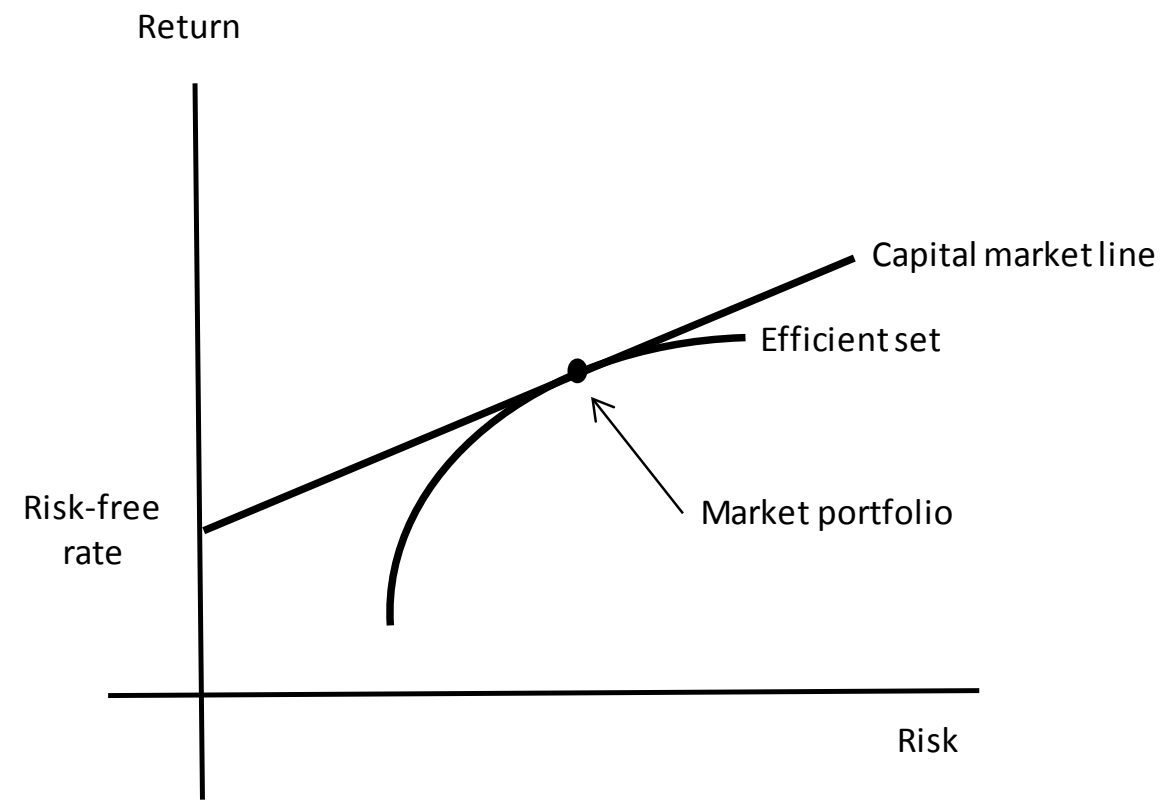

For reaching the market equilibrium in capital markets, Sharpe (1964) made a number of assumptions for the CAPM in line with Markowitz $(1952,1959)$ and Tobin (1958): 
- Investors follow the Markowitz's model for making investment decisions

- The same rate of interest for lending and borrowing applies to all investors

- All investors have homogenous expectations about expected returns, risk and correlations

In the CAPM, more reward means to take more risk. Because investors want the extra return without the extra risk, they compete in the market for getting an edge in the form of information, but will not succeed because of the mechanism of full competition. CAPM presupposes informational efficiency, which means that prices of securities accurately reflect all available information (Fama, 1970).

In other words, efficient markets are based on the rational expectations of investors. Lucas (1978) says that rational expectations and informational efficiency are the same. Or in the words of Read:

This full information and full rationality approach in finance at the time was analogous to the rational expectations school of macroeconomics that was also developing in the 1960s (Read, 2013, pp. 102-103).

John Muth (1961) has introduced the notion of rational expectations, i.e. expectations from common sense, as opposed to adaptive expectations. His argument is that the professor of economics cannot predict, say, hog cycles better than the 'hog' (sc. pig) farmer. Why is it an expectation from common sense? Because the argument tells us that economists would be rich, if they could make better predictions than hog farmers (McCloskey, 1998).

Concerning the relation between the CAPM and the efficient market hypothesis, Fama (1970) notes that market efficiency cannot be determined without a theory about equilibrium. So, without presupposing equilibrium there can be no expected return or risk, and no stochastical predictability of investments. Fama (2011) calls 
the logical combination of the postulates of market efficiency and market equilibrium the joint hypothesis problem. Fama echoes Hayek (1948), a representative of the Austrian School, who sees foresight (predictability) and equilibrium as a tautology. But Hayek differs from Fama, in the sense that he does not believe in either part of the tautology:

It appears that the concept of equilibrium merely means that the foresight of the different members of the society is in a special sense correct (Hayek, 1980/1948, p. 42).

Hayek's argument against predictability is that economic knowledge is dispersed over the individual participants, which do not know each other's future plans. It is the market which brings the participants and their plans together: the precise outcomes that will emerge are unpredictable.

\section{After the CAPM: APT and the Anomalies}

The successor of the CAPM is the Arbitrage Pricing Theory (Ross, 1976). The Arbitrage Pricing Theory (APT) allows for other explanatory factors than CAPM's market risk to determine an expected risk and return of a security. The assumptions of APT are deliberately less restrictive than those of the CAPM:

The primary goal of Stephen Ross' APT was to relax the implication that every investor chooses the same portfolio. Instead, Ross sought to show that different investors balance a spectrum of possible risks (Read, 2013, p. 130).

Unlike the CAPM, which reduces all risk to market risk, APT is an open empirical theory, therefore it does not prescribe the factors that determine the expected risk and return of a security.

Another follow-up of the CAPM is that it has inspired a large empirical branch in investment theory, which tries to identify anomalies in the efficient market hypotheses. A number of well-known anomalies is found by Fama and Kenneth 
French (1993), which find three factors explaining returns: besides the market, factors for small caps, and stocks with low book to price ratio, explain returns. Haugen (1995) suggests to renew modern finance, because empirical research such as Fama \& French (1993) shows that value stocks have a higher return and a lower risk, thus, Markowitz's portfolio theory and the CAPM are not appropriate.

Besides Fama \& French's findings, lots of articles have been written about for example calendar, momentum, and behavioural anomalies. It would be out of scope to treat and comment all the anomalies.

Though a criticism of the econometric method would be out of scope here as well, it is nevertheless important to express my general view about the predictability of the anomalies found. The empirical investigations attack the theory of the efficient market in the sense that all available information is not reflected, but that does not mean that financial markets have become predictable. If some predictable pattern in the stock market continues to exist, than market forces will exploit the phenomenon, after which it stops existing: it resembles the non-existing 100 euro notes on the pavement near your house. Yet, full arbitrage is not possible, because arbitrage is restrained by the availability of capital needed and the capacity to take the risks involved (Shleifer \& Vishny, 1997). In so far, the efficient market theory is useful because it explains that easy profits will not persist because of arbitrage. Of all the information available, the easy piece with clear consequences will be priced in for sure.

\section{8: Option Theory}

Another important part of modern investment theory is option theory. Option theory treats the valuation of contingent claims and calculates the price of the right to sell or buy for example a stock against a specified price in a specified period. The theory of options has, of course, a history beyond the conventional notion that it is invented in the 1970s. Anecdotal history tells the story of the Greek 
philosopher Tales of Milete (624-545 BC) who successfully bought options on olive-presses (Aristotle, 1912). De la Vega (1688) describes the mature option market of the Amsterdam stock exchange of the seventeenth century. Bachelier (1900) seems to have made the first serious contribution on the pricing of options. Another early contributor to option theory is Vinzenz Bronzin in 1908:

While Bronzin's approach is more pragmatic than Bachelier's, every element of modern option pricing can be found: risk-neutral pricing, no-arbitrage and perfecthedging pricing conditions, the put-call parity, and the impact of different distributional assumptions on option values. [....].

His equation [...] is closer to the Black-Scholes formula than anything published before Black, Scholes and Merton (Zimmerman \& Hafner, 2006, p. 238).

Because it is out of scope for the dissertation, the reader who is interested in a more detailed history of option theory is referred to Espen Haug \& Taleb (2011).

The credit for finding the solution for option pricing is usually given to Fisher Black \& Myron Scholes (1973) and Robert Merton (1973b). Option theory enables to calculate the price of a right to buy or sell, for example, a stock at a specified price in a specified period. Black \& Scholes (1973), and Merton (1973b), are able to solve the formula for option pricing, because they demonstrate that the risk premium above at the risk-free rate becomes irrelevant in the derivation of the formula. Black \& Scholes (1973) derive a formula for the pricing an option from both the CAPM and the so-called put-call parity. The Black \& Scholes formula is mathematically explained in the Appendix B of the Chapter. Robert Merton (1973b) further demonstrates that the Black-Scholes formula depends on risk-free arbitrage: a position in stocks can dynamically hedge the exposure of an option. The option price equals the statistically expected loss of the hedging portfolio. A long position in stocks, the holding of stocks, hedges a call option, a short position, selling the stocks short, hedges a put option. 
Black, Scholes, and Merton imply that the risk of the underlying investment of an option can be estimated. Otherwise, a market maker in options faces possible losses, because the dynamic hedging costs can be higher than expected if the risk realized is higher than the risk expected. Yet, in the line of my argument in the dissertation, both risk and return are unpredictable: an option price reflects a subjective level of risk. Option theory has had a huge influence on the practice of option trading and the development of financial engineering (Hull, 2009/1997, p. 277). Some, though, are critical of the perceived role of option theory in practice: Derman \& Taleb (2005) do not believe that dynamic hedging is possible in practice: instead, they claim that static hedging with the put-call parity is the fundamental principle of option pricing.

Option theory differs from the CAPM in the expected level of the return of risky assets: option theory expects that risky assets return the risk-free rate, CAPM expects a risk premium above the risk-free rate. Option theory and the CAPM differ in the expected return of risky assets because they relate differently to risk preferences. Option pricing is independent of risk preferences, and founded on the principle of risk neutral valuation (Hull, 2009/1997, p. 289). The CAPM adheres to the preference of risk aversion, the economic principle of a diminishing marginal utility, and expects therefore that a higher return should compensate for a higher risk.

How does option theory fits in with portfolio theory? Using options as a part of Markowitz's optimization procedure to find the efficient set of portfolios seems problematic, because the usual risk measure of portfolio theory, the standard deviation, is not fit for the asymmetric risks that options create. Of course, the semi-deviation, which measures the volatility below a certain level, say, the riskfree rate, could be an alternative measure of risk, but the semi-deviation as well would measure the risk too imprecise when options are involved. On top of that, call options should profit from the risk premium of stocks, which the option 
formula excludes. And, put options should have a disadvantage because of the positive tilt of the risk premium. Furthermore, option theory and portfolio theory differ fundamentally in handling risk: portfolio theory merely postulates theoretical risk reduction as a free lunch, instead option theory delivers a derivate that guarantees risk reduction at a real cost. Markowitz's optimization procedure is simply not designed to handle the asymmetric risk profile of options, the absence of the risk premium in option theory, and the fundamentally different character of risk reduction of options.

\section{9: The Criticisms of Modern Finance}

Leaving the anomalies of the efficient market out, the criticisms of modern finance can be loosely divided in five groups:

- the ideological criticism of financial markets as merely gambling and at times harmful

- the criticism of the conventional theory of statistics used in finance by Benoit Mandelbrot: fractal finance

- the criticism of the rationality of financial markets: bubble theory

- the behavioral criticism of the rationality of individuals in finance

- the criticism of evolutionary finance

Please note that another important criticism of non-mainstream economics about uncertainty and unpredictability will be treated at length in the next chapter. The criticism about uncertainty is about applying statistics for prediction when information lacks about the future.

\section{The Ideological Criticism}

In 2008, the credit crisis has led to a crisis on the financial markets. In the credit crisis, the ideology of the rational market as advocated by Alan Greenspan and others was put to a reality check. Central in the rational market theory is the 
efficient market hypothesis (Fox, 2009, p. xiii). To repeat, the efficient market hypothesis assumes rational expectations, which mean that individuals cannot have better information than the markets as such. Yet, though the efficient market hypothesis is a useful scientific model, it has become overstretched by the idea that the rational market comes up with the true price:

Financial markets knew best. They moved capital from those who had it to those who needed it. They spread risk. They gathered and dispersed information (Fox, 2009, p xii).

The rational market theory makes forget that the financial market is "a devilish thing" as well (ibid., p. xv). Cassidy explains that stability of markets is an illusion because of the effect of bubbles:

Once a bubble begins, free markets can no longer be relied on to allocate resources sensibly or efficiently. By holding out the prospect of quick and effortless profits, they provide incentives for individuals and firms to act in ways that are individually rational but immensely damaging - to themselves and others (Cassidy, 2010/2009, p. 9).

To repeat the earlier findings of the Chapter, the perspective that financial markets resemble gambling is an old one, witnessed by the De la Vega's (1688) eyewitness report of the Amsterdam Exchange since the $17^{\text {th }}$ century. In the $19^{\text {th }}$ century, investing in stocks is still associated with gambling, but the vernacular science of financial investments turns it into a scientific topic, in which rational behaviour in the form of self-control and the study of information, is promoted as the key to successful investing (Preda, 2006). A part of vernacular science includes probabilistic and abstract reasoning and results in the efficient market theory. The perspective that financial markets can be managed by a scientific approach is enforced since modern finance emerged in the 1960s. 
Though investing has unknown probability distributions, unlike gambling in a casino, investing is perceived unlike gambling because it has a rationale, arguments to believe that it is worthwhile. Marieke de Goede (2005) takes a genealogical approach to investigate finance:

A genealogy, in short, is a practice of criticism that is motivated by finding insecurities and uncertainties in that which is represented as stable, coherent, and self-perpetuating (De Goede, 2005, p. 14).

Finance is now seen as a respectable scientific and public subject, instead of as gambling associated at times with fraud and manipulation; money on a savings account should be replaced by investments because it should, instead of doing nothing, be activated in the financial market. The change of a perception of finance from gambling to a rational activity took time and was forced by the emergence of the financial markets, of which the actors needed respectability:

In the eighteenth and nineteenth centuries, the lack of a conceptual distinction between 'finance', 'gambling', and 'speculation' increasingly became an obstacle to the respectability of trading in stocks, shares, and credit certificates. A separation between gambling and finance became thinkable only through a prolonged political, cultural, and legal struggle surrounding the meanings and boundaries of 'the financial sphere' and the character and behavior of 'financial man' (De Goede, 2005, p. 48).

The genealogical approach underpins that the distinction of finance and gambling is merely political, ideological, instead of based on the nature of financial markets (De Goede, 2005, p. 114).

A defence of modern finance against the ideological criticism and others criticisms to come, lies partly in the meaning of rational markets. Finance adheres to the idea of rational markets as guided by arbitrage. The mechanism of arbitrage is a paradigm of modern finance. The mechanism of arbitrage implies that some individual participant on the financial needs to be rational and to apply arbitrage. 
But for successful arbitrage not all market participants need to be rational, even a lot of irrational participants are no problem. Moreover, it is a relative simple form of rationality because arbitrage is about getting a riskless profit and merely assumes that people want more money without an extra risk. The rationality of arbitrage is far less demanding than the rationality of a regular market equilibrium with regard to the requested number of rational participant and the complexity of their preferences. Of course, the argument of arbitrage has its limits, merely plain information can be arbitraged, but noise cannot be arbitraged.

Whether the benefits of financial markets outstrip its negative sides, is outside the scope of the dissertation. Yet, the question how people should cope with financial markets is relevant: suffices the current scientific approach of modern finance to handle investments, or does its epistemology, or lack thereon, urge us to look for alternative solutions?

\section{Benoit Mandelbrot's Fractal Finance}

As McCloskey (1990) says, facts constrain stories. The point now is what facts are in finance, because statistical theories frame facts. Benoit Mandelbrot (1963a, 1963b, 2004 with Hudson) starts by undoubtable facts, mostly simple data, that of prices on financial or commodity markets and their fluctuations. Then, the choice of statistical theory, normally the theory coupled to that of the normal distribution, decides the description of the data for the purpose of explanation or prediction. But Mandelbrot proposes an alternative statistical theory, the theory of fractals, to explain the data on financial and commodity markets. The normal distribution cannot explain Black Monday, the stock market crash 19 October 1987, on which the American stock market tumbled 29.2\%: the odds of such a crash were less than $10^{50}$ following a normal distribution (Mandelbrot \& Hudson, 2004, p. 4). The received view of perceiving facts on the financial markets can also offer an alternative explanation for the credit crisis of 2008: 
The worldwide market crash of autumn 2008 had many causes: greedy bankers, lax regulators and gullible investors, to name a few. But there is also a lessobvious cause: our all-too-limited understanding of how markets work, how prices move and how risks evolve (Mandelbrot \& Hudson, 2008/2004, p. xi).

So, the assumptions behind the models in finance are a part of the problem. The assumptions pretend that risk is manageable, but turbulence is normal, not abnormal, in financial markets. According to Mandelbrot's \& Hudson's, modern finance is problematic:

Financial economics, as a discipline, is where chemistry was in the sixteenth century: a messy compendium of proven know-how, misty folk wisdom, unexamined assumptions and grandiose speculation (ibid., p. xv).

Mandelbrot recognizes Bachelier's random walk process as an important theory about the behaviour of financial markets, but replaces the normal probability distribution for the so-called stable Paretian probability distribution, because the statistical properties of the distribution of price changes on financial markets are deviant from the normal distribution in the sense the distribution are to peaked, leptokurtic, to be normal distributions:

Despite the fundamental importance of Bachelier's process, which has come to be called 'Brownian motion', it is now obvious that it does not account for the abundant data accumulated since 1900 by empirical economists, simply because the empirical distributions of price changes are usually too 'peaked' to be relative to samples from Gaussian populations (Mandelbrot, 1963a, p. 394, his italics).

Besides the probability distribution, the efficient market hypothesis which relates information, news, and fundamental analysis to the pricing of a security is flawed (Mandelbrot \& Hudson, 2008/2004, p. 8). An example of a flaw in the efficient market theory is that prices are dependent, instead of independent as presumed by the efficient market hypothesis, though the dependence of prices does not mean that the prices become predictable (ibid., pp. 11-12). Another point is that the efficient market hypothesis does not explain the endogenous working of financial 
markets, despite that a financial market does more than reflecting outside events (ibid., p. 21).

The hypothesis of efficient processing of information seems not adequate as an explanation of the behaviour of financial markets, but that does not mean that markets become predictable. For Mandelbrot, the economic significance, i.e. that prediction is impossible, of the efficient market hypothesis remains valid. Though he does not believe in the prediction of markets, having a theory which explains the behaviour of financial markets is worthwhile as a product of science. His theory is not meant to make money, but to enlarge wisdom.

Mandelbrot uses the mathematics of so-called fractals, a small geometric form which accumulates to some complex phenomenon, to simplify the understanding of phenomena:

A fractal, a term he [Mandelbrot] coined form the Latin for 'broken', is a geometric shape that can be broken into smaller parts, each a small-scale echo of the whole. The branches of a tree, the florets of a cauliflower, the bifurcations of a river -all are examples of natural fractals (Mandelbrot \& Hudson, 2008/2004, p. $\mathrm{xx}$, my insertion).

The theory of fractals translates into the Pareto probability distribution, a power law distribution. Vilfredo Pareto (1848 - 1923) is an Italian economist, sociologist, statistician among others (Kirman, 2008). Other names for the Pareto probability distributions are 'Lévy', 'Lévy-Mandelbrot', 'L-stable', or 'stable Paretian'. The Pareto probability distribution is known in popular terms by the $20 / 80$ rule, for example 20 percent of the habitants tend to earn 80 percent of the income in a country. The Pareto distribution is featured by a long (fat) tail. Mandelbrot sees the Pareto distribution as representative for many economic phenomena: 
It is not very seriously questioned, however, that the law of Pareto represents very satisfactorily, not only the 'tail' of the distribution of personal income, but also the tails of the distributions of firm sizes and of city sizes (Mandelbrot, 1963b, p. 421).

Mandelbrot \& Hudson (2008/2004, p. viii) distinguish three forms of randomness: mild, slow, and wild. Modern finance assumes mild randomness which is represented by the normal distribution. But finance is represented by wild randomness:

My contribution was, foremost, to recognize that in turbulence and much else in the real world, roughness is no mere imperfection form some ideal, not just a detail from a gross plan. It is of the very essence of many natural objects -and of economic ones (Mandelbrot \& Hudson, 2008/2004, p. 125).

The Paretian distribution which Mandelbrot proposes for finance, has next to its wild randomness, another feature which explains theories and ideas about financial markets: the feature is that the Paretian distribution creates the illusion of causality in stock markets:

I shall also show the following: when the 'spontaneous activity' of a system is ruled by a Paretian process, the causally structural features of the system are likely to be very much more hidden by noise than is the case where the latter is Gaussian. Causal structures may even be totally 'drowned out'. On the other hand, Paretian noise generates all kinds of 'patterns' that seem to be perfectly clear-cut but have no value for purposes of prediction (Mandelbrot, 1963b, p. 422).

Another consequence of accepting the Paretian distribution is that the standard deviation becomes useless, because it becomes infinite and that usual statistical methods do not work:

It is well known that second moments are heavily used in statistical measures of dispersion or of 'standard deviation' and in 'least-squares' and 'spectral' methods. Hence, whenever the considerations of Section V are required to explain the 
erratic behavior of sample second moments, a substantial portion of the usual methods of statistics should be expected to fail (Mandelbrot, 1963b, p. 432).

If the Paretian distribution holds, Markowitz was wrong in choosing the standard deviation as the measure of risk. The same goes for the CAPM, which assumes normal distributions.

If probability distributions are Paretian, normal statistical analysis does not work, and finance becomes a science of interpretation like history. Interestingly enough, Mandelbrot resembles Von Mises, Keynes, Knight, but has another theory, though from a source from within economics by the ideas of Vilfredo Pareto:

Broadly speaking, a pattern is scientifically significant and is felt to have chances of being repeated, only if in some sense its 'likelihood' of having occurred by chance is very small. This kind of significance is obviously to be assessed with the help of the tools of statistics; unfortunately, those have been mostly designed to deal with Gaussian alternatives and, when the chance alternative is Paretian, they are not conservative or 'robust' enough by far. [....]. But, when one works in a field where the background noise is Paretian, one must realize that one faces a burden of proof that is closer to that of history and autobiography than to that of physics (Mandelbrot, 1963b, p. 433).

Though the Paretian distribution is probably not predictive because it is difficult to establish the relevant parameters, it has a chance of being a better theory for the random walk hypothesis than a normal (or lognormal) distribution (ibid., p. 434). But the consequences of the Paretian distribution are huge: regular statistics do not function anymore, the standard deviation is no longer representative, and that is negative for the assumed benefits of diversification. Concerning the CAPM, it cannot function because it assumes the normal distribution.

The implications of Mandelbrot's theory are devastating for finance and regular statistics: stocks are riskier than modern finance postulates, diversification does not work well, and volatility is unstable. Initially, Mandelbrot's ideas spread in 
finance but were ignored later on. Though for example Taleb (2007), a popular opponent of modern investment theory and its related risk management practices, adheres to Mandelbrot's ideas, Mandelbrot remains in the periphery of finance theory.

\section{Charles Kindleberger: Bubble Theory}

Kindleberger (1910-2003) is an economic historian specialized in the history of financial crises. Financial crises happen over and again. To mention some crises in the last 20 years: the Euro-crisis of 2012, the credit crisis of 2008, the dotcom bubble of 2001, the Russia-crisis of 1999, and the Asia-crisis of 1997. He persuades that crises are a part of the economic system and have always existed. Crises relate to the behaviour of individuals and crowds. His book (1978) Manias, Panics, and Crashes: A History of Financial Crises received the recommendation of Paul Samuelson:

Sometime in the next five years you may kick yourself for not reading and rereading Kindleberger's Manias, Panics, and Crashes. Paul A. Samuelson (Kindleberger, 2000/1978, front-page).

According to Minsky (2000/1978, pp. 14-15) bubbles and their bursting behave according to a fixed pattern:

- A bubble starts with a displacement, an outside shock to the economy, such as a new invention, or a monetary or political change. A displacement brings opportunities for profits.

- Then credit expands and fuels the boom. Banks can expand the money supply.

- Prices rise and a positive feedback develops by the entry of new investors.

- The bubble reaches the stage of euphoria. Speculation is added now. Profits are overstated.

- Then the bubble bursts with the accompanying panic. 
- Finally, the phase of capitulation is reached. People have lost their confidence.

Kindleberger uses the ideas of Herman Minsky (1982) for the interpretation of bubbles. He dubs Minsky's model as a classical economic model:

Indeed, in its emphasis on the instability of the credit system, it is a lineal descendant of a model, set out with personal variations, by a host of classical economists including John Stuart Mill, Alfred Marshall, Knut Wicksell, and Irving Fischer. Like Fisher, Minsky attached great importance to the role of debt structures in causing financial difficulties, and especially debt contracted to leverage the acquisition of speculative assets for subsequent resale (Kindleberger, 2000/1978, p. 14).

Kindleberger (ibid., p. 13) does, of course, not mean that all economic upswings end up in bubbles. Nor does he seek some regularity in time to make predictions, like some business cycle theorists have done, such as Kitchin's cycle of 39 months, Juglar's cycle of 7/8 years, Kuznets's cycle of 20 years, and Kondratieff's cycle which is set off by major inventions.

Kindleberger's research shows that people behave irrational in markets. Though the normal behaviour of people is rational, he relates manias and panics with temporal mass irrationality or mob psychology (ibid., p. 26). More precisely, irrationality of the whole can emerge from rational individual behaviour. He calls the idea that rational individuals act rationally as a whole a fallacy of composition, but emergence of irrationality out of individual rational behaviour happens on other occasions as well, for example when people stand up in a theatre to get a better view, with the consequence the irrational outcome that other people get a worse view (ibid., p. 217). Individuals can be irrational as well, as for example illustrated by the phenomenon of cognitive dissonance: people possessing the same facts use different personally coloured 'theories', and reach other conclusions on the basis of the same facts (ibid., p. 2018). Another example is that information is interpreted 
on different levels of expertise, because not everyone is an expert, which leads to irrationality in the market.

\section{Robert Shiller}

A countermovement against the efficient market emerges along with an accent on the forgotten role of irrational behaviour (Read, 2013, p. 190). Shiller (1981) is an early opponent of the efficient market theory:

If, by the 1980s, there began to emerge a healthy skepticism of the efficient market hypothesis, there remained few convincing alternatives. The chief advocate for the popularization of a reconsideration of the efficient market hypothesis was Robert Shiller. He helped motivate a movement to construct a new behavioral paradigm to the pricing of financial instruments (Read, 2013, p. 188).

Shiller (1981) finds that the variance of stocks was 5 to 13 times higher than an efficient market model would predict on the basis of available information. Later on, Schiller (2000) explains in his book Irrational Exuberance that the bull market of the 1980s and 1990s is not related to economic fundamentals. He expresses a number of possible causes for the irrational rise of the stock market in the 1980s and 1990s such as positive feedback models of for example adaptive expectations, cultural causes such as media and 'new era'-thinking, and behavioural causes such as herd behaviour.

Shiller (with Akerlof, 2009) works in the tradition of Keynes's ideas of psychology of markets in the form of animal spirits, which translate into bubbles and Keynes's beauty context which emphasizes the reflexivity of markets, meaning that the market reacts to itself. So, to explain behaviour, Shiller replaces rational economic explanations by psychological and cultural explanations such as confidence, fairness, bad faith, money illusion, and stories. In Chapter 5 about the culture of finance Shiller will also be treated. 


\section{Behavioral Finance}

In a general critique of the assumption of rational behaviour in economics, Herbert Simon (1955) introduces the alternative notion of bounded rationality. Bounded rationality limits the knowledge and the calculative ability of the decision maker. Yet, in classical decision theory the rational decision maker is omniscient, and does not make mistakes in his calculations. A consequence of bounded rationality is that a decision maker does not optimize, but satisfices. The school of behavioural finance continues the path of bounded rationality. Behavioural finance has emerged as a new branch of investment theory. Behavioural finance studies the psychology of decision making in investing.

Daniel Kahneman (2011) distinguishes two systems of thinking, the heuristic automatic mode (system 1) and the effortful attentive mode (system 2). The heuristic mode of thinking that leads to behavioural biases, is the regular way of thinking, the effortful mode can cope with heuristic biases. Behavioural finance has found that "Uncertainty is poorly represented in intuition, as well as in perception (Kahneman, 2003, p. 701)." Thus, translating intuition into personal probability is troublesome. For example, Amos Tversky and Kahneman (1974) show that judgment under uncertainty is biased, because of the heuristics of representativeness, availability, and anchoring. The heuristic of representativeness shows that probability judgments based on representativeness, similarity, can be flawed, because probability is influenced by a larger array of factors than mere similarity. The availability bias explains why people misjudge the probability of an event merely because some events can easily be brought to mind. The anchoring bias suggests that judgments are influenced the information presented by the starting point.

Relevant to the investment decisions in the dissertation, is that historical data often are available as a basis to form judgments about future returns, and that investment theory 'prescribes' how to present the data, so the judgment under uncertainty is 
biased at a higher level than presented by the examples of the behavioural theory of Tversky and Kahneman. Behavioural finance also offers an explanation why decision makers about investments are captured by the perspective of investment theory. Ellsberg (1961) described the phenomenon of ambiguity aversion, meaning that people do not like uncertainty and prefer a presentation which is more elaborate, which is more generally described as attribute substitution (Kahneman \& Frederick, 2002). Specifically to investment theory it means that models are the attribute of substitution:

[...] our brain has the latent tendency to substitute complex problems with less complex problems. And these less complex, though often mathematically rigorous problems are then resolved as a substitute solution for the complex problems. That is called attribute substitution (Kocken, 2012, p. 18).

Shefrin (1999, p. xii), a prominent writer on behavioural finance, expresses as an important lesson from behavioural finance that investors should be aware of their overconfidence. Research shows that most investors make psychological errors in making investment decisions. Overconfidence also applies to the use of models in finance, and, in addition, investors select the information that confirms their earlier choice, in other words their opinions about the model are flawed by the confirmation bias (Rosenberg \& Kocken, 2013). Behavioural finance offers insight in why investors keep on using flawed model by the cognitive biases that pervade their thinking. Because of their critique of expected utility theory, Tversky and Kahneman develop an alternative, the prospect theory (1979). For a summary of behavioural finance see Shefrin (1999), Kahneman $(2003,2011)$ and Thaler \& Sunstein (2008).

Gerd Gigerenzer (2000) is a critic of the program of heuristics and biases as performed by Tversky \& Kahneman and others. In the conventional program of behavioural finance heuristics and biases are measured against the norms of probability theory. But the norms of probability theory adapted are doubtful: 
What in the heuristics-and-biases literature is called the "normative theory of probability' or the like is in fact a very narrow kind of neo-Bayesian view that is shared by some theoretical economists and cognitive psychologists and to a lesser degree by practitioners in business, law, and artificial intelligence (Gigerenzer, 2000, p. 244).

Gigerenzer advocates the notion of ecological rationality: "[...] rationality that is defined by its fit with reality (Gigerenzer et al, 1999, p. 5)." Ecological rationality is a form of bounded rationality and explains how people cope with uncertainty. The difference between ecological rationality and the rationality assumed by Tversky and Kahneman is that ecological rationality does not focus on probability theory. Ecological means that, instead of probability theory, the structure of the environment is relevant for decision making. The specific circumstances determine what is rational to include in decision making; probability theory is too general and therefore unfit in most circumstances of practical decision making.

As a case of pluralism in economics (Mirowski \& Sent, 2002), the emergence of behavioural finance is a favourable development. Measured by the tolerance of academic economics and finance, behavioural finance is successful as an alternative for neoclassical finance. It seems that no other alternative investment theory has gained the level of attention in the academic world and in the investment practice as behavioural finance.

The explanations of behavioural finance and the rhetoric of investment theory for the shortcomings of investment theory coincide. For example, the metaphor that investing is mathematical statistics, could be seen as a framing effect, which makes us see in a particular way. Behavioural finance also shows the conceptual difficulty that people experience with statistics. The strength of behavioural finance that is predicts individual behaviour in decision making, and that it has a coherent theory why people act the way they do. But as a matter of prediction of 
the financial markets, behavioural finance has no other status than other alternative investment theories, that is behavioural finance explains but does not predict financial markets.

\section{Evolutionary Finance}

Finance applies statistics to financial markets as a top-down approach. Statistics assumes that the underlying process produces random outcomes which are stable, stochastically predictable. The arguments for applying the stability hypothesis in

finance, such as rationality, the efficient market hypothesis, arbitrage, fundamental intrinsic value, and market equilibrium have been discussed in Chapter 2 and will be analyzed at length in Chapter 3 of the dissertation. An alternative approach, however, is evolutionary finance, which is a bottom-up approach that starts with the behaviour of market participants. The evolutionary approach has gained ground in a variety of sciences. The constitutional metaphor of the bottom-up approach of evolutionary finance is evolutionary biology, instead of mechanical and statistical physics as used in neoclassical finance. The Santa Fe Institute, a research center for the evolutionary approach in science which was founded in 1984, has played an important role in promoting the evolutionary approach.

Evolutionary finance grounds in a number of related theories, such as dynamic systems, reflexivity, complexity, and emergence.

\section{Dynamic systems}

In evolutionary finance, financial markets are seen as a dynamic system, in which the causes of changes are endogenous, from within the system (Beinhocker, 1997/1996, p. 100). For example, the interaction between the behaviour of individuals can result in a self-fulfilling prophecy (Merton, 1948). An example of a self-fulfilling prophecy starts with a false rumor that a certain bank has become insolvent, after which some customers of the bank start to withdraw their money, and attract other customers who do the same. Then the bank will indeed fail if left 
by itself, and the prophecy has become self-fulfilling. A self-fulfilling prophecy is a wrong belief about a situation, but becomes true because behaviour adapts to the belief:

[...] public definitions of a situation (prophecies or predictions) become an integral part of the situation and thus affect subsequent developments. This is peculiar to human affairs (Merton, 1948, p. 195).

A self-fulfilling prophecy is fed by feedback loops: in the example of the insolvency of the bank, first merely some people believe the rumor, then the withdrawal of money creates more believers and then the bank will fail. But such feedback loops are no part of neoclassical economics.

Another form of a feedback loop is the phenomenon of reflexivity. An advocate of reflexivity is George Soros $(1987,2008)$. Soros $(2008$, p. 10) describes reflexivity as a circular feedback loop between beliefs and reality: beliefs and reality influence each another. Thus, beliefs in financial markets influence the economic reality, which reinforces beliefs. In contrast to neoclassical finance, in Soros's view the beliefs of market participants are flawed by definition because of uncertainty. The consequence of the flawed beliefs of financial markets leads to boom and bust cycles, in which optimism feeds optimism and pessimism feeds pessimism. In the case of financial markets, optimism and pessimism are instantaneously visible in the market prices.

\section{Complexity}

Another aspect of evolutionary finance is that financial markets are a non-linear system, which can result in complex patterns (Beinhocker, 1997/1996, pp. 102107). Thomas Schelling (1978), an economist awarded the Nobel prize in 2005, investigates how individual behaviour relates to the outcome at the collective level: if individual behaviour depends on individual behaviour of others then the collective behaviour is probably not merely the total of the behaviour of the 
individuals, but can have an unexpected outcome because of the interaction between the behaviour of individuals.

Schelling performed his analysis in a qualitative manner, that is non-mathematical.

We will see later on in the current paragraph that mathematical and experimental methods also have been employed in evolutionary economics and finance.

An important feature of complexity is the so-called tipping point, in which the state of a system changes to another form, like water transforms to ice (Popescu, 2015, p. 14). A tipping point in financial markets is for example a regime shift from a bull to a bear market. The technique of agent based modelling enables to simulate bottom-up behavior and interaction between individual agents including institutions, and helps understanding tipping points in finance and macroeconomics. Networks, this is the way in which agents are connected and cluster in finance and economy, are an important part of understanding tipping points and complexity in general (Beinhocker, 2006). Agent based modelling reveals how small changes in individual behavior because of interactions between people and institutions can lead to dramatic consequences on the collective level. Agent based modelling also gives clues for policymakers how to interfere in the markets to improve stability or to reduce the consequences of tipping points.

\section{Emergence}

Emergence is typically an output of bottom-up processes: "The movement from low-level rules to higher-level sophistication is what we call emergence (Johnson, 2001, p. 18)." Emergence results from complex systems: the properties of the total of individual parts cannot be reduced to the properties of its individual parts, but emerge from the interaction of the individual parts. Examples of emergence are phenomena such as ant colonies, life, consciousness, intelligence and music (Hofstadter, 1979). 
Formal and experimental evolutionary finance

I will now illustrate some insights from formalized models of evolutionary finance in which the behaviour of the market is simulated. Hommes (2005) reviews heterogeneous agent models in which agents have a bounded rationality and act behavioural on simple heuristics. Another feature of the agents is that they are heterogeneous, which means that they act differently, for example, agents belong to different groups such as fundamental investors, who focus at intrinsic value and chartists, who focus at trends in historical market prizes. The models stemming from heterogeneous, bounded rational agents result in dynamic behaviour and can explain phenomena such as excess volatility and stock market crashes. Though the evolutionary finance models reviewed by Hommes (2005) are equipped with sparse bottom-up assumptions, they succeed in explaining the actual outcomes of the financial markets.

Evolutionary finance is also suited for an experimental approach, meaning that a group of people can engage in an experiment as financial market participants. Such an experiment enables to study for example the relationship between past performance and expectations on financial markets. Anufriev, Hommes, and Philipse (2013) found in explaining the outcomes of such an experiment that a model in which behaviour switched between adaptive and trend-following behaviour was best suited to explain negative and positive feedback loops.

\section{Evaluation of the Criticisms}

Concerning the depth of the rival theories of finance, they all have substantial backing and empirical proofs which can compete scientifically with modern investment theory. The ideological criticism uses elements of behavioural and bubble criticisms and combines them with the criticism on free markets in general. In essence, the ideological criticism says the investing in financial markets resembles gambling which is politically sanctioned. Investment theory seen is through the ideological perspective is a rationalisation of a dubious practice. The 
ideological criticism is related to a broader criticism of money, economics and free markets, which lays outside the scope of the dissertation.

Mandelbrot provides within the rational mathematical tradition an alternative statistical theory, which does not predict, but can model market phenomena in ways not possible for traditional statistics. Fractal finance models seemingly predictive patterns in financial markets, such as wild volatility and bubbles. His contrasting statistical theory shows that empirical research in finance is highly theory-driven, meaning here the regular theory of statistics. To my opinion, Mandelbrot's theory is a very interesting alternative to modern finance, because it provides a better model for the dynamics at financial markets. Interestingly enough, Mandelbrot's idea roots in economics as well: the economics of Vilfredo Pareto.

The bubble theory of Minsky has a different view on markets than modern finance and explains bubbles and their bursting. Kindleberger has a large collection of historical cases which follow Minsky's model. Shiller also advocates the bubble theory of markets. The bubble theory is persuasive and recognizable. The theory does not claim predictability of markets.

Behavioural finance is insightful and explains behaviour of market participants and their behaviour. The interesting point of behavioural finance is that it has a coherent way of explaining what investors do wrong in given circumstances. Behavioural finance can also explain why investors keep on using investment theory, or more general, keep on believing in prediction of financial markets by the biases of attribute substitution, the confirmation bias, and overconfidence.

Evolutionary finance seems a promising alternative finance theory because the evolutionary approach can explain the instability of financial markets out of the bottom-up outcomes of the interaction of market participants. The evolutionary 
approach to finance is meant to explain and does not intend to predict -evolutionary biology does not claim to predict either. Evolutionary finance does not exclude other alternative finance theories and relates to elements of the alternative investment theories of behavioural finance, bubble theory, and fractal finance which have been treated in the current chapter of the dissertation. 


\section{Appendix 2A: The Mathematical Statistics of Diversification}

To understand Markowitz's theory, some technical exploration into the characteristics of the probability distribution and the effect of diversification is helpful. Mathematical statistics provides the mechanics of diversification. The expected return, the standard deviation, and the correlation specify the statistics in portfolio theory. The expected return is the average, or mean return: 'expected' here has a statistical meaning. 'Expected' in statistics means the value of the investment return at $50 \%$ of the probability mass of the distribution (Newbold, 1984). The probability mass is the sum of the probabilities of possible returns: the mass amounts by definition to $100 \%$. For investors, the probability of actually receiving the mean return is low: expected return is not to be understood as a return that an investor can anticipate. The expected return is to be understood as a minimal (or maximal) return with a probability of 50\% -if the probability beliefs are correct. The standard deviation, the risk, measures the dispersion around the expected return. The standard deviation weights larger deviations more heavily, because the deviations from the mean are squared in the calculation of the standard deviation. In Figure 2.3 a probability distribution of returns relates to Markowitz's efficiency criteria of mean and standard deviation: 


\section{Figure 2.3: Probability distribution, standard deviation and mean}

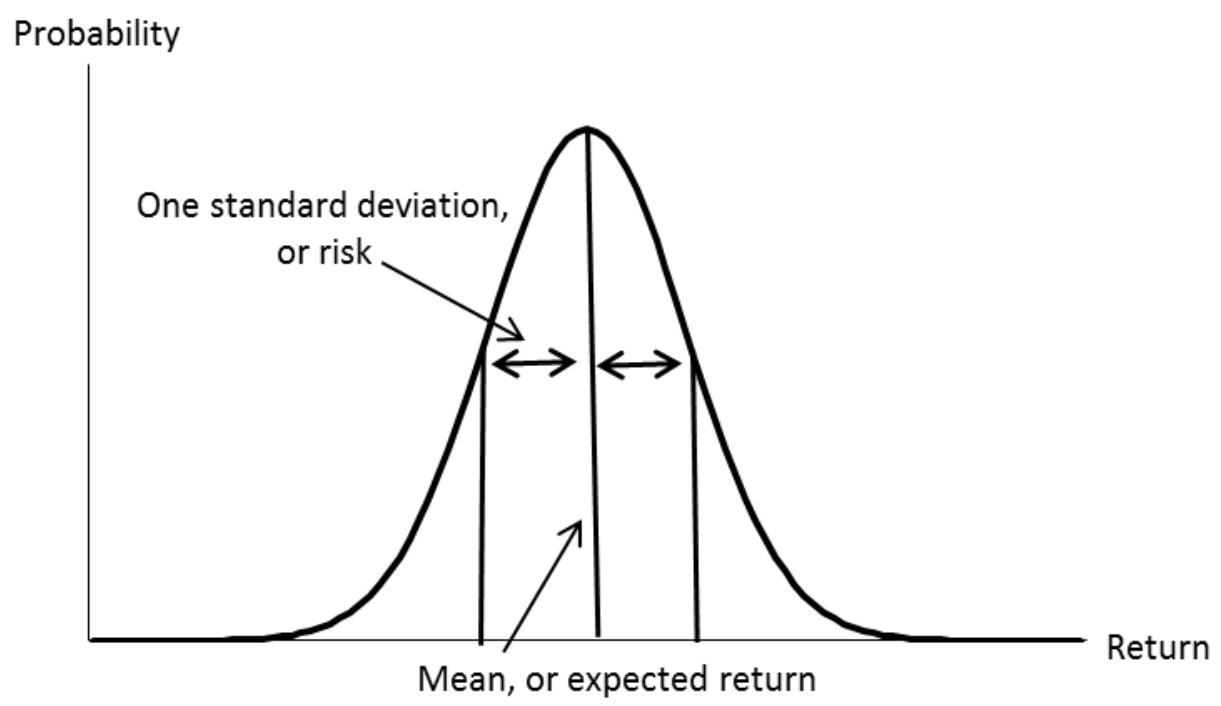

In portfolio theory, expected return and risk are the main characteristics of an individual security. Yet, at the portfolio level, the interplay between individual securities matters: the correlation coefficient expresses the interplay. The correlation coefficient measures the linear relationship between two variables on a scale from -1 to +1 . If the correlation coefficient is 1 , the returns always move together in the same direction, a correlation of 0 implies that returns have no linear relationship, and a correlation coefficient of -1 means that the returns behave in exactly the opposite direction.

I will now show mathematically, how diversification between two securities lowers the risk of a portfolio (Bodie et al, 1989). Please note that the calculation of the expected return of a portfolio does not demand an elaboration: it is simply a weighted average of the expected returns of the securities in the portfolio. The standard deviation, or risk, of a portfolio of two securities is formulated as: 


$$
\sigma_{p}=\sqrt{\left(w^{2} \sigma_{a}^{2}+(1-w)^{2} 2 \sigma_{b}^{2}+2 w(1-w) \rho \sigma_{a} \sigma_{b}\right)}
$$

The symbols used mean:

$\sigma_{p}=$ standard deviation of portfolio $\mathrm{p}$

$\sigma_{a}=$ standard deviation of security a

$\sigma_{b}=$ standard deviation of security $\mathrm{b}$

$w=$ weight in the portfolio

$\rho=$ correlation coefficient

The formula illustrates that the risk of a portfolio depends on the standard deviations of the individual securities, $\sigma_{\mathrm{a}}$ and $\sigma_{\mathrm{b}}$, their weights, $\mathrm{w}$, and on the correlation coefficient, $\rho$, between them. A correlation coefficient less than one, yields a diversification benefit. If the correlation coefficient is at its upper limit of 1 , the portfolio risk equals the weighted sum of the risks of the individual securities. If the correlation coefficient is at its lower limit of -1 , the third term of the right hand side of the formula becomes negative, and leads to an extraordinary diversification benefit. In the special case that the correlation coefficient is 0 , the third part of the right hand side of the formula becomes 0 . Then, the risk of a portfolio reduces to the weighted risks of the individual securities:

$\sigma_{p}=\sqrt{\left(w^{2} \sigma_{a}^{2}+(1-w)^{2} \sigma_{b}^{2}\right)}$

The formula with two securities illustrates the general mechanism of diversification. Yet, normally an investor can diversify over a lot of securities. 
Now, the analysis will cover the effect of an increasing number of securities. To benefit the analysis, the securities in the portfolio have the same risk, expected return, and correlation coefficient.

If the correlation coefficient between the securities is 0 , the risk of the security, divided by the square root of the number of securities, determines the portfolio risk:

$\sigma_{p}=\frac{\sigma_{a}}{\sqrt{n}}$

Symbol used:

$n=$ number of securities

The formula demonstrates that more securities lower the risk of the portfolio. Because of the square root in the denominator of the right hand side of the formula, an extra security in the portfolio leads to a diminishing marginal risk reduction. If the number of securities is high, the portfolio risk eventually becomes 0 . A correlation coefficient of 0 resembles an insurance-like activity, because insurance assumes individual risks to be more or less uncorrelated. The formula expresses the law of large numbers, which reduces the risk of an insurance-like portfolio to 0 at a large number of 'cases'.

The general formula for calculating portfolio risk, when securities have the same expected return, risk, and correlation coefficient, is expressed like (Bodie et al, 1996/1989, p. 227): 


$$
\sigma_{p}=\sqrt{\frac{1}{n} \sigma^{2}+\frac{n-1}{n} \rho \sigma^{2}}
$$

Supposing that the risk of the individual security is $25 \%$, Figure 2.4 illustrates the risk reduction as a function of the number of securities and the correlation coefficient:

\section{Figure 2.4: Idiosyncratic risk reduction with correlation coefficients of 1,0} and -1

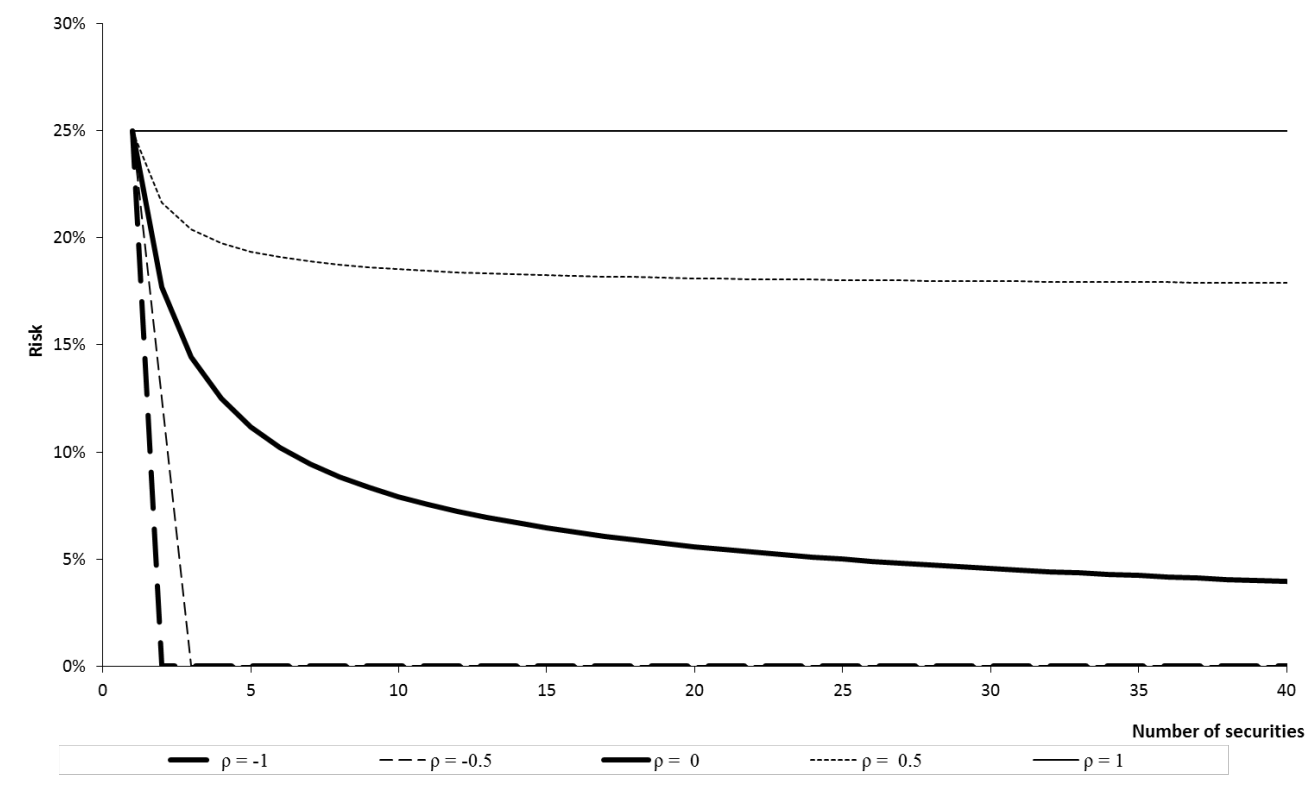

Risk reduction by adding securities is at its most effective at a correlation coefficient of 0 , represented in Figure 2.4 by the solid bold line. Suppose the correlation coefficient would be -1, then two securities would be enough for an optimal risk reduction to 0 . If the correlation coefficient is 1 , risk cannot be reduced. At a level of correlation coefficient of 0.5 , some risk reduction can be achieved, but the optimal reduction requires fewer securities in comparison with a 
correlation of 0. Diversification cannot eliminate all variance (Markowitz, 1952, p. 79). To repeat, in modern portfolio theory the correlation coefficient is above 0 , because merely idiosyncratic risk of individual securities can be diversified, in contrast to systematic risk, that is market, risk. The idealized example of the mechanism of diversification meant to clarify Markowitz's notion of risk reduction, but of course the correlation coefficients between securities will mostly differ, just like their expected risk and return. 


\section{Appendix 2B: The Black \& Scholes Option Formula}

The Black \& Scholes option formula deals with the so-called European option, in which a holder can only exercise his right to buy or sell at the end of the duration. In the formula, Black \& Scholes assume that the stock will not deliver a dividend. The Black \& Scholes call option formula is expressed as (based on Hull, 1997):

$C=S N\left(d_{1}\right)-X e^{-r T} N\left(d_{2}\right)$

where:

$d_{1}=\frac{\ln (S / X)+\left(r+\sigma^{2} / 2\right) T}{\sigma \sqrt{T}}$

$d_{2}=d_{1}-\sigma \sqrt{T}$

The meaning of the symbols used is:

$C \quad=$ the current value of the call option

$S \quad=$ the current price of the stock

$N(\quad)=$ the standardized normal distribution

$N(d)=$ the probability that a drawing out of $N$ is lower than $d$

$X \quad=$ the exercise price of the call option

$e \quad=$ the base of the natural logarithm

$r \quad=$ risk-free interest rate (continuously compounded with $e$ )

$T \quad=$ time to maturity

ln = natural logarithm

$\sigma=$ standard deviation, or risk, of the stock price 


\section{Chapter 3}

\section{Investment Theory, Probability}

\section{Theory, and Uncertainty}

"Some consumers of Statistics want to believe in the magic of Statistics. They view Statistics as a type of Mathematics and are offended to learn that judgment is involved at all. [....]. It is the very fact that judgment is a part of a statistical analysis that opens the question of the honesty of Statistics (Paul Velleman, 2008, p. 8)."

\section{1: The Logos of Probability}

Markowitz's portfolio theory $(1952,1959)$ claims that it can handle investment decisions with mathematical statistics. The focus of the current Chapter directs primarily at the logos, the rational arguments, of the probability beliefs used in portfolio theory, and also at the logos of the probability beliefs used in the Capital Asset Pricing Model (CAPM), the continuation of Markowitz's theory.

The Chapter intends to contribute to the literature on the rhetoric of investment theory by a close reading of the epistemological assumptions in Markowitz's primary texts $(1952,1959)$. The discussion has partly been presented in Pistorius (2014). Markowitz argues that statistical knowledge about future investment returns is possible by invoking the theory of subjective probability. My intention is to explain his reasoning and its implications, and to show that the assumption of stochastical predictability in portfolio theory is flawed. To repeat, stochastical predictability differs from deterministic predictability: ideally, a deterministic 
prediction results with certainty in a particular outcome; a stochastical prediction yields certainty over a range of possible outcomes with probabilities attached.

Though Markowitz's theory has, of course, been commented extensively in the literature, the epistemological part about the probability beliefs in portfolio theory seems to be ignored, because the validity of subjective probability theory is a basic assumption in mainstream economics. To repeat, Markowitz's portfolio theory is not merely of theoretical importance: the theory constitutes investment and risk practice in the financial sector and its beliefs have caused financial damage, as discussed in Chapter 1.

\section{2: Probability Beliefs in Portfolio Theory}

We now investigate Markowitz's ideas about predictability of investment returns. He explores in Portfolio Selection (1959) how beliefs about investment returns fit in with the theory of probability. Markowitz founds his theory on mathematical statistics: he assumes that '[ ...] 'beliefs' or projections about securities follow the same probability rules that random variables obey (Markowitz, 1999, p. 5)."

Portfolio theory applies decision theory, which uses probability theory to predict or estimate the probability of future outcomes. He leaves no doubt that investment returns are uncertain, and that security analysts cannot overcome uncertainty:

Uncertainty is a salient feature of security investment. Economic forces are not understood well enough for predictions to be beyond of doubt or error. Even if the consequences of economic conditions were understood perfectly, non-economic influences can change the course of general prosperity, the level of the market, or the success of a particular security. [....]. We are expecting too much if we require the security analyst to predict with certainty whether a typical security will increase or decrease in value. [....]. The existence of uncertainty does not mean that careful security analyses are valueless. [....] Carefully and expertly formed judgments concerning the potentialities and weaknesses of securities form the best basis upon which to analyze portfolios (Markowitz, 1991/1959, p. 4). 
Markowitz says that investment returns are uncertain because of multiple economic and non-economic causes, and that both causes are difficult to predict. As a consequence, a security analyst cannot predict the future return of a security with certainty. Nevertheless, the judgment of a security analyst possesses information about the value of a security and its uncertainties. Markowitz's predecessors, to repeat, Williams (1938) and Graham et al (1934), provide the theories about how to calculate the value of a security. Portfolio theory as such has no economic theory about the beliefs applied, and can, to repeat, incorporate any economic theory which brings in probability beliefs.

Markowitz prefers probability beliefs derived from the judgments of security analysts above probability beliefs based on merely historical observations:

Portfolio selection should be based in reasonable beliefs about future rather than past performances per se. Choice based on past performances alone assumes, in effect, that average returns of the past are good estimates of the 'likely' return in the future; and variability of return in the past is a good measure of the uncertainty of return in the future (Markowitz, 1991/1959, p. 14, his italics).

He says that reasonable probability beliefs include the past, but that merely past performance seems no good guide for future returns. But what is his argument that past returns do not equal future returns? It could be the instability of the probability distribution of returns, which results from the multiple economic and non-economic causes. Obviously, he seems to believe that the economic theory of valuation, as used by security analysts, offers a solution to overcome instability, because the market price of a security will, in the future, return to its 'equilibrium' value. But the judgments of security analysts, derived from the theory of valuation, are not directed at making a probability distribution. So, the judgments need to be translated into beliefs about the probability distribution of investment returns. 
Markowitz explains the nature of a probability belief by a hypothetical example, in which a person wins the same amount of money by choosing between two probability events $(1959$, p. 27). He has to choose between the event that 'it does not rain tomorrow', and the probability of $80 \%$ of winning in a fair 'wheel of fortune'. He will try to discover the probability that 'it does not rain tomorrow': if the probability is lower than $80 \%$, he will choose the wheel of fortune, otherwise he will choose the event that 'it does not rain tomorrow'. The example illustrates that a probability could be estimated by comparing an uncertain event with a lottery, or more generally with a probability distribution. The outcome of the comparison is, that some probability distribution will be felt as representative for the uncertainty of an event. The lottery metaphor that a probability belief is a lottery bet, is the centrepiece of the theory of subjective probabilities.

Markowitz founds his theory on subjective, also called personal, probabilities. Subjective and objective probabilities refer to two distinct theories:

In an objectivist theory, probability is seen as an attribute of the world. [....]. In the subjectivists' theory, probability is [...] an indication of a particular individual's state of knowledge about uncertain events (Langlois, 1982, p. 7).

In portfolio theory, objective probabilities could only refer to the past, of which Markowitz is, to repeat, no advocate because the past lacks the judgment of security analysts. Neoclassical economics, to which Markowitz' theory belongs, adheres to subjective probability for the purpose of decision theory. Adherents to subjective probability theory, should not be confused with adherents to methodological subjectivism in economics (Langlois, 1982, p. 2). Adherents to methodological subjectivism, such as economists of the Austrian school, found economics on subjective people, but reject subjective probability theory: the Austrian school does not consider statistics an appropriate tool for most decisions in economics. 
The distinction between objective and subjective, personal, probabilities relates to the various approaches within probability theory. Three ways to estimate probabilities are: 'classical', 'frequentist', and 'subjective' (Gilboa, 2009). The objective classical approach assumes that all outcomes are equally probable. The 'principle of indifference' explains the classical approach: the indifference stems from an absence of a good argument to give unequal probabilities to the outcomes. The classical approach restricts to artificial producers of chance, such as the tossing of a coin. The objective, frequentist approach regards the probability of an event as the relative frequency in past observations: the approach is the statistics often used in empirical science. The frequentist approach assumes that the law of large numbers applies: the law of large numbers relates the mean of a sample to the mean of its population. The subjective, or personal approach, uses the machinery of probability to explicit beliefs, but mixes with the probabilities of the objective approaches as well (Savage, 1954).

Subjective probabilities are connected to the term 'Bayesian': yet, 'Bayesian' has two meanings. First, in statistical theory 'Bayesian' means that new evidence updates probabilities. The Bayesian method of updating probabilities, or Bayes's rule, competes with the frequentist approach in making inductive inferences on observations. But, because frequency theory can express Bayes's rule, the rule "does not serve as the distinguishing mark of subjectivism (Langlois, 1982, p. 7)." The second meaning of 'Bayesian' refers to the theory in neoclassical economics that, to maximize expected utility, subjective probabilities can replace objective ones when objective probabilities are not available (Gilboa et al, 2008).

Leonard Jimmy Savage (1954) has invented the paradigm that it is rational to maximize expected utility with subjective probabilities, if objective probabilities are absent. He deducts from a number of axioms of rational behaviour, that decision making under uncertainty maximizes expected utility under subjective probabilities. In short, the axioms of rational behaviour treat completeness and transitivity of preferences between bets, separation of tastes from believes, and the 
sure thing principle, in which the dominance of the probability distribution suffices to make a decision (Gilboa et al, 2008). Savage prefers the term 'personal' instead of 'subjective' probability. In the dissertation, both terms mean the same.

Savage claims that the use of personal probabilities is consistent with rational behaviour. Thus, even if probability beliefs are uncertain or vague, it is rational to quantify the beliefs to make decisions. It is rational, because all the information the decision maker possesses, is fully expressed in a rational, coherent manner. The economic literature has heavily debated Savage's assumptions of rationality. Gilboa (2009, Chapter 12) presents an overview of the criticism of Savage's axioms. For Markowitz's portfolio theory, especially the Ellsberg paradox seems relevant, because the paradox distinguishes objective and personal probability. The paradox (Ellsberg, 1961), also known as ambiguity aversion, shows that decision makers favour objective to personal probability. To illustrate the paradox, Ellsberg designs two experiments, of which one will be explained here. A decision maker has to choose between two urns: he is told about one urn that $50 \%$ of its balls are red, and 50\% black. The other urn contains red and black balls as well, but no indication is given about the distribution between the red and black balls. Probably, the decision maker estimates the personal probabilities for the second urn as $50 \%$ probability of red, and $50 \%$ of black. Thus, the probabilities of taking a red ball in the first and the second urn are both $50 \%$. But if the decision maker is asked to choose to take a red ball out of urn one or urn two to win a price, he will prefer urn one. The reason for preferring urn one, is that objective probability feels safer than personal probability. Ellsberg's critique undermines Savage's personal probability theory: if people would maximize expected subjective utility, they should be indifferent to choosing between urn one and urn two.

To repeat the findings of Chapter 2, Simon (1955) introduces a general critique of the assumption of rational behaviour in economics with the alternative notion of bounded rationality. Bounded rationality limits the knowledge and the calculative 
ability of the decision maker. Yet, in classical decision theory the rational decision maker is omniscient, and does not make mistakes in his calculations. A consequence of bounded rationality is that a decision maker does not optimize, but satisfices. The school of behavioural finance continues the path of bounded rationality. Behavioural finance has found that "Uncertainty is poorly represented in intuition, as well as in perception (Kahneman, 2003, p. 701).” Thus, translating intuition into personal probability is troublesome. Rationality for the goal of maximizing expected utility in economics relates to ethics: the source of ethics in economics is the notion of utility, which belongs to the virtue of prudence (McCloskey, 2006). I will in Chapter 4, among other things, contrast the 'prudence-only' rationality of investment theory to the broader virtue approach of McCloskey (1996a, 1996b, 2006). Klamer's approach of values $(2003,2014)$ will be treated in Chapter 5 and provides an alternative for the rationality as assumed in neoclassical economics as well. Klamer's value approach inquires which values are important and relates the values to decision making.

\section{3: Markowitz's Defence of Personal Probabilities}

Markowitz uses personal probabilities in portfolio theory for two reasons: it is rational coherent behaviour, and personal probabilities in investment theory should be able to predict. But rational behaviour and predictability are two separate arguments: rational behaviour does not automatically lead to predictability. Moreover, Savage's approach is about rational decision making, and not necessarily about prediction:

[...] that theory [the theory of personal probability] is a code of consistency for the person applying it, not a system of predictions about the world around him (Savage, 1954, p. 59, my insertion).

Let us now discuss how Markowitz (1959) relates the use of personable probabilities to predictability. Out of his discussion of Savage's theory of 
probability beliefs, he draws, in his own words, 'two morals', about the predictability of the probability distributions of investment returns, which I will fully quote to support my analysis:

1. The existence of personal probabilities does not necessarily imply that, as of the moment, the individual is positive that his beliefs are 'good beliefs.' He may admit the possibility that he currently is either always overoptimistic or always overpessimistic, or in some other way subject to biased judgment. However, the idea of probability beliefs does imply a belief in an ability to learn with time and experience, to end a long life of predictions and constant education without substantial biases on the whole.

2. The connection between objective and subjective probabilities is quite close. We noted that they mixed on a par with each other in the calculation of expected utility. The discussion of this section indicates another connection. To assert that some physical experiment has a .6 probability of producing a result $A$ is to assert that , if this physical experiment is carried out and if a large number of other physically independent experiments (of the same or different kinds) all with a probability of .6 of producing some particular (though perhaps different) result $A$ are also carried out, then the relative frequency of $A$ will almost certainly be .6. Similarly, to assert that a .6 personal probability is associated with an event is to assert the belief that it is virtually certain that the relative frequency of correct predictions among a set including this and a large number of other psychologically independent events is .6. Thus personal probabilities and subjective probabilities are connected via the notion of relative frequency in the long run (Markowitz, 1991/1959, pp. 272-273, his italics).

Concerning Markowitz's first conclusion about subjective probability beliefs: he acknowledges that the exercising of personal probabilities can lead to a possibly biased judgment, but it also implies an ability to learn and become better at estimating. His argumentation suggests that he assumes that the probability distribution of investment returns can be known in principle, which means the probability distributions are not ontological, but merely epistemological uncertain. Yet, we have to ask ourselves under which conditions it is possible to learn about probability beliefs. We can learn about the 'real' probability distribution of investment returns if the distribution is stable over time, or if changes of the properties of the distribution can be predicted. I claim in the dissertation that the 
probability distribution of investment returns is unstable. The current Chapter 3 reviews the arguments against stability in economic phenomena such as returns on financial markets and presents my own thought experiment about the possibility of predictability for investment theory.

Let us illustrate what happens when probability beliefs are updated. The probability distribution of investment returns applied could differ manifest from reality, if an event such as the credit crisis of 2008 with the accompanying negative results in the financial markets occurs. In the reasoning of Markowitz, the answer to a phenomenon like 2008, is the Bayesian approach of updating the probability estimates with the new information. But if the probability distribution of investment returns is unstable, updating does not lead to a better level of predictability, because the 'real' distribution of investment returns cannot be learned. It is uncertain how often a crisis such as in 2008 will happen, and a next crisis could turn out even worse than 2008. If the probability distributions would be stable, objective probabilities will become available if the number of observations becomes sufficiently high: both the frequentist and Bayesian method in statistics can be used to update and learn from new observations, both by a bigger sample. My conclusion is that personal probability beliefs about investment returns can only by coincidence be correct. So, though personal probabilities sharpen the intuition, they are not able to stochastically predict the future. The second conclusion of Markowitz claims that personal probabilities are like subjective probabilities by the notion of relative frequency in the long run. Meant here is seemingly that personal probabilities are individual probabilities, and that subjective probabilities are the accumulation of personal probabilities. My view is that he refers to Savage's point that the personalistic view of probability can contain reasonable, objective views like the notion of relative frequency as well:

I would reply [to the critics] that the personalistic view incorporates all the universally acceptable criteria for reasonableness in judgment known to me and 
that, when any criteria that may have been overlooked are brought forward, they will be welcomed into the personalistic view (Savage, 1954, p. 67, my insertion).

My view is that the second moral of Markowitz about similarity to the objective frequency approach is only valid if the probability distribution is, again, stable, or if the changes in the distribution are predictable. Just like his first moral, the second moral is founded on the assumption of stability. So, both his morals to justify the predictability of probability beliefs in investment theory depend on the assumption of stability, or the ability to predict changes in the stability.

\section{4: Investment Theory after Markowitz's Portfolio Theory}

After Markowitz discovers how a rational investor would act, the next logical step in price theory is a formulation of the market equilibrium of expected risk and return. As discussed in Chapter 2, Sharpe (1964) builds a theory of equilibrium in financial markets, aka Capital Asset Pricing Model (CAPM). Sargent (2008) explicitly assumes stability in the pattern of the economic variable to be predicted. Rational expectations for financial markets mean that the possible levels of risk and return on investments are known in advance, which is the same as stochastical predictability of risk and return.

Equilibrium in financial markets is a centrepiece of the CAPM, and an indirect subject of portfolio theory, since Markowitz refers to valuation theory as a source for probability beliefs. Valuation is a sort of equilibrium theory: the theory assumes that the price of a security converges to its fundamental value. Portfolio theory and the CAPM act at different levels: portfolio theory is about the individual investor and CAPM about the market equilibrium, which emerges if all investors act like Markowitz assumes rational investors to do. Yet, one can wonder, whether the personal probability approach of Markowitz differs much from the rational expectations approach of the CAPM, because both assume stochastical prediction. If the stability of expected risk and return of investments is a valid assumption, 
portfolio theory resembles CAPM in its assumptions about statistics: Markowitz's rational investor learns about the real probability distribution during time, and, CAPM's rational investor can be wrong as well, though not on average in the sense of the market as whole, and also learns during time.

\section{5: Evaluation of Probability Theory in Investment Theory}

To select investment portfolios in practice, portfolio theory needs reasonable future expected returns and standard deviations (Markowitz, 1952). Reasonable expected returns and standard deviations are to be interpreted here as more or less predictive. Markowitz leaves no doubt that he believes in the predictability of investment returns in the long run: to repeat, he concludes about personal probabilities that they are meant to learn and end up "[...] without substantial biases on the whole" (1959, pp. 272-273).

Markowitz's theory is grounded on Savage's personal probability theory. Savage claims that rational, coherent behaviour under uncertainty is accompanied by personal probabilities. Whether the probability beliefs are reliable is not relevant for Savage's theory: the use of probability beliefs is rational and coherent. So, even if the probability belief about the investment returns in Markowitz are unreliable, in other words, if investment returns are stochastically unpredictable, it still makes sense to use mathematical statistics in investment theory because it is rational and coherent behaviour under uncertainty. Of course, one has to agree with the definition of rationality applied by Savage to support his ideas. To repeat, the economic literature has heavily debated Savage's assumptions of rationality, and in the Chapters 4 and 5 the scope of rationality as presumed in neoclassical economics, which reduces ethics to utility, will be broadened.

What does using personal probabilities mean for decision making about investing? Let us start with answering the question by investigating the meaning of the 
expected return of an investment. With personal probabilities, one cannot claim as certain that the probability of obtaining an expected return of stocks of, say, $8 \%$, will be met at least be in $50 \%$ of the cases: but the claim would be true by definition, if the expected $8 \%$ was the true expected return. An investor could choose a more cautious estimate to compensate for the uncertainty. But even so, the correction could be too small: one seldom encounters expected stock returns below those of bonds. The estimation of risk cannot be sure either, though one could prefer a more cautious estimate of that as well. But how can one judge what is the right amount of caution (think about the credit crisis of 2008)? The point of the examples is that the gap between the personal and the true probabilities cannot be bridged. Using personal probability beliefs in practicing investment theory gives us no clue to the probability as such, that the probability beliefs for the investment decisions are correct.

Moreover, using personal probabilities in investment theory leads to the same decisions as using true probabilities: so, personal 'becomes' as it they were true outcomes, which is dangerous, because decision making on the basis of personal probabilities should not be done merely by statistics: under the condition of uncertainty, statistics should merely be used to explicit the intuition to help making judgments.

Let us now turn to the theory of objective probabilities, which founds the CAPM. Davidson $(1982 / 1983,1991,2009)$ has extensively reflected on current mainstream economics in the light of Keynes's ideas about uncertainty in economics. Current mainstream economics, following Paul Samuelson and Robert Lucas, believes that economics must be based on stochastical predictability:

Acceptance of the presumption of an ergodic economic environment is often rationalized by the necessity of developing economics as an empirically based science (Lucas and Sargent, 1981, pp. xi-xii). Indeed, Samuelson (1969[b], p. 184) has made the acceptance of the 'ergodic hypothesis' the sine qua non of the scientific method in economics (Davidson, 1991, pp. 132-133, my insertion). 
The ergodic hypothesis as used in the rational expectation hypothesis assumes that objective probability information is available from past data. The assumption is needed to make economics a predictive science, and accommodates a stochastical framework as well. But Davidson's point is that past data cannot predict the future stochastically because unexpected changes occur.

In Knight's (1921) terms, stochastical predictability is a situation of 'risk'. But if probability distributions in investing are unstable, portfolio theory and the CAPM ignore Knight's situation of 'uncertainty', in which probabilities are unknown. Statistics under Knightian uncertainty becomes 'merely' an argument. Yet, defenders of the received view express the general climate which ignores Knightian uncertainty:

While the distinction between risk and uncertainty so defined is often encountered in the literature, its role until recently has been reduced to the ceremonial: economists, especially those working in the neoclassical tradition, invoke the distinction only in order to rule out uncertainty. [....]. There are good reasons for doing so. In the modern theory of choice, subjective probabilities are derived from agents' orderings over lotteries. Hence (simplifying) it follows that to deny the existence of subjective probabilities is to deny that agents are able to choose consistently among lotteries. Most economists are unwilling to do without the assumption of consistent choice (LeRoy \& Singell, 1987, p. 395).

The discourse of modern investment theory as part of mainstream economics simply does not 'allow' talking and thinking in Knightian terms of risk and uncertainty, as risk means the same as uncertainty.

I agree that explicating subjective probability beliefs is useful: I am not against using statistics in investment theory. If the beliefs are not predictive, they still could be of importance for theoretical or historical analysis: they can imagine the future as a probabilistic restatement of intuitive beliefs, or of alternative scenario's, or analyse the past, with historical observations as input. But I reject that 
investment returns are stochastically predictable, and therefore the role of the portfolio theory cannot be prediction. It follows that if one does not believe in the 'weaker' form of predictability of subjective probability, the stronger assumption of predictability as assumed in the CAPM cannot be persuasive either.

I cannot prove by empirical induction that predictability is impossible: the proof of unpredictability lies outside the paradigm of statistics, because statistics assumes that the substrate that produces probability outcomes, is stable. The inference to the best explanation that investment returns are unpredictable, seems more persuasive than the arguments of investment theory that personal or objective probability beliefs predict. Savage's second pillar of rationality seems a better argument for using Markowitz's theory. But rationality in economics has been critically evaluated as well, and cannot 'repair' unpredictability.

\section{6: Risk against Uncertainty}

Current investment theory advocates the use of probability theory, though other currents within economics and finance oppose such a use of probability theory and prefer the assumption of uncertainty instead of calculable risk. The literature review about uncertainty in economics and investment theory in the current paragraphs, treats the accounts of statistics and uncertainty, arguments against predictability, and both its meanings for investment theory. In paragraph $3.10 \mathrm{a}$ thought experiment about predictability in investment theory will be treated. The review selects those ideas from the history of uncertainty in economics which are relevant for investing. The ideas about uncertainty in economics apply to investment theory as well because investment theory is a part of economics. The review does not intent to present a full history of uncertainty in economics, for which is referred to Wubben (1993). From the history of economics the ideas of Knight, Keynes, and Von Mises seem the most relevant for the review. The three economists appear to be the most important writers on uncertainty for investment 
theory: besides being original thinkers, they involve statistics in their analysis. Of course, other important economist thought about uncertainty in economics too. For example, Hayek (1948) highlights the dispersion of knowledge as a cause of unpredictability: yet, to my knowledge, Hayek, though introducing a theory for the reason of economic uncertainty, did not specifically criticize the traditional philosophy of probability. Israel Kirzner (1985), another Austrian economist, highlights the role of the entrepreneur, whose alertness discovers opportunities in the market. Kirzner, however, did not seem to have reflected on the philosophy of probability either. Shackle (1955) did write extensively on probability and economics, but does not seem to contribute to my purpose of clarifying the nature of stochastical predictability in investment theory after treating the ideas of Knight, Keynes, and Von Mises.

For the same reasons of being original thinkers and involving statistics in their analysis, I will discuss the ideas of Deirdre McCloskey and Nassim Taleb from the contemporary literature about uncertainty.

\section{Knight}

Knight was probably the first to treat uncertainty in economics in full. $\mathrm{He}$ distinguishes uncertainty from risk (Knight, 2009/1921, p. 9). Risk means that probability distributions are known, and uncertainty that probability distributions are unknown, because a group of instances, representative cases, lacks to make a probability distribution:

The practical difference between the two categories, risk and uncertainty, is that in the former the distribution of the outcome in a group of instances is known (either through calculation a priori or from statistics of past experience), while in the case of uncertainty this is not true, the reason being in general that it is impossible to form a group of instances, because the situation dealt with is in high degree unique (Knight, 2009/1921, p. 121). 
He classifies three types of probabilities, of which 'a priori probability' and 'statistical probability' belong to risk, and the third one 'estimates' belongs to uncertainty:

1. A priori probability. Absolutely homogeneous classification of instances completely identical except for really indeterminate factors. This judgment of probability is on the same logical plane as the propositions of mathematics [...].

2. Statistical probability. Empirical evaluation of the frequency of association between predicates, not analysable into varying combinations of equally probable alternatives. It must be emphasized that any high degree of confidence that the proportions found in the past will hold in the future is still based on an a priori judgment of indeterminateness. [....]. The main distinguishing characteristic of this type is that it rests on an empirical classification of instances.

3. Estimates. The distinction here is that there is no valid basis of any kind for classifying instances (ibid, pp. 115-116, my emphasis).

Artefacts, like games of chance, produce $a$ priori probability. A priori probability allows mathematical calculation because cases are perfectly homogeneous. The second type of 'statistical probability' cannot achieve the perfection of a priori probability:

The practical difference between a priori and statistical probability seems to depend upon the accuracy of classification of the instances grouped together. In the case of the die, the successive throws are held to be 'alike' in a degree and a sense which cannot be predicated of the different buildings exposed to fire hazard (ibid, p. 112, my italics).

A priori probability and statistical probability share the law of indifference. The law of indifference implies that the outcome of an individual case is unpredictable, because the specific causes which decide the outcome of an individual case are unknown. If the outcomes of a group of particular cases within a larger group differ from the ones of the larger group, a narrower group of instances could achieve statistical probability once again: therefore, an insurance company may differentiate the insurance premium for groups of customers, depending for 
example on age or residence. To predict a priori probability and statistical probability, we assume that the probability distribution remains the same for some future period (ibid, p. 116). Knight calls the third type 'estimates' uncertain because the probabilities are unknown. Estimates lack past cases to represent the future because the case at hand is unique: the lack of a "[...] valid basis of any kind for classifying instances (ibid, p. 116)" separates estimates from a priori and statistical probability. For Knight, economics is in general about uncertainty, and thus about estimates.

\section{Keynes}

Keynes expresses his account of probability in A Treatise on Probability (1921).

He was the first to treat the idea of logical probability in a structured way (Hacking, 2009/2001, p. 144). A theory of logical probability aims to discover the probability, the degree, to which an inconclusive argument is true. In the theory, the probability that an argument is true depends on the knowledge available. Keynes's theory of logical probability belongs to the logic of inductive inference, and assumes objective probability (Cottrell, 1993). Logical probability is nowadays less in vogue than subjective or objective probability.

How does Keynes's early probability theory relate to his later account of uncertainty in economics as displayed in The General Theory of Employment, Interest, and Money (1936)? Efforts to connect Keynes's probability theory to his later account of uncertainty have led to various interpretations (Dequech, 2000). His probability theory and his economics seem to be independent: Keynes (1936, 1937) hardly refers to his theory of logical probability in his account about radical uncertainty in economics (Cottrell, 1993, pp. 42-43). An explanation for the apparent discontinuity between The Treatise on Probability (1921) and The General Theory (1936) could be, that it is merely a matter of accent: The Treatise is about induction from assumed true premises, while The General Theory investigates the content of the premises as such (Cardim de Carvalho, 1988, p. 72). 
For the dissertation, his theory of logical probability seems less relevant, because it assumes true premises.

In the current section I will use Skidelsky's account (2009a, pp. 84-86) of Keynes's breakdown of types of probability in A Treatise on Probability. Keynes distinguishes cardinal and ordinal probability, and irreducible uncertainty. A cardinal probability is a measurable probability such as used in insurance. In economic practice, cardinal probability is rare. Ordinal probability ranks probabilities of events in a qualitative sense, and occurs often. Ordinal probability lies in between the certainty of cardinal probability and irreducible uncertainty. Irreducible uncertainty neither compares nor quantifies: its probability is unknown. In The General Theory he leaves out ordinal probability to contrast irreducible uncertainty to cardinal probability as proposed by mainstream economics. Keynes's account of irreducible uncertainty is comparable to Knightian uncertainty:

By 'uncertain' knowledge, let me explain, I do not mean merely to distinguish what is known for certain from what is only probable. The game of roulette is not subject, in this sense, to uncertainty $[\ldots]$. [...]. The sense in which I am using the term is that in which the prospect of a European war is uncertain, or the price of copper and the rate of interest twenty years hence, or the obsolescence of a new invention, or the position of private wealth-owners in the social system in 1970. About these matters there is no scientific basis on which to form any calculable probability whatever. We simply do not know (Keynes, 1978/1937, pp. 113-114).

\section{Von Mises}

Like Knight and Keynes, Von Mises distinguishes between known and unknown probabilities:

There are two entirely different instances of probability; we may call them class probability (or frequency probability) and case probability (or the specific understanding of the sciences of human action) (Von Mises, 2012/1949, p. 107). 
Von Mises relates class probability to a closed group such as a lottery or a table of mortality, in his words:

[...] We know or assume to know, with regard to the problem concerned, everything about the behaviour of a whole class of events or phenomena; but about the actual singular events or phenomena we know nothing but that they are elements of this class (ibid, p. 107).

Class probability resembles Knight's situation of risk and Keynes's cardinal probability. In class probability a pool diminishes risk to the certainty of some cost. Von Mises stresses that the pooling of the class is essential, but not the calculus of probability:

Insurance, whether conducted according to business principles or according to the principle of mutuality, requires the insurance of a whole class or what can reasonably be considered as such. Its basic idea is pooling and distribution of risks, not the calculus of probability. [....]. The calculus of probability is mere by-play.

This is clearly evidenced by the fact that the elimination of hazardous risk by pooling can also be effected without any recourse to actuarial methods (ibid, $\mathrm{p}$. 109).

Von Mises coins uncertainty 'case probability'. Case probability applies to economic phenomena. Non-economic examples of case probability are elections, sport games, and medical advice. Von Mises relates uncertainty to multi-causality:

Case probability means: we know, with regard to a particular event, some of the factors which determine its outcome; but there are other determining factors about which we know nothing (ibid, p. 110).

Knight, Keynes, and Von Mises share a common denominator about uncertainty in economics: risk, cardinal probability, or class probability merely deal with a small group of economic phenomena, and, uncertainty and case probability reign over economic phenomena. 


\section{7: Arguments for Uncertainty in Economics}

\section{Knight}

What are Knight's arguments for his idea that economic phenomena belong to uncertainty instead of risk? Let us start with the metaphor that he proposes for economics in order to include the dynamics of economic phenomena. He perceives economic phenomena as organic rather than mechanical:

Perhaps the most straightforward way to approach Knight's philosophy is to recall the distinction he constantly makes between the mechanical and the organic (biological) frameworks. Mechanistic thinking views human behaviour and institutions as static, machine-like entities, whereas organicistic thinking invokes notions such as change and process. [....]. Knight's distinction between risk and uncertainty contrasts the mechanical and organic domains (Langlois \& Cosgel, 1993, p. 458).

Only if economics takes uncertainty as its premise, it would acknowledge Knight's organic metaphor. The organic metaphor brings change to the fore. Economic change is the major theme of Joseph Alois Schumpeter: "[...] he [Schumpeter] strongly felt that capitalism was unique in history because of its ceaseless and selfgenerated changefulness (Heilbroner, 1997/1996, p. 299, my insertion).”

Schumpeter stresses the role of the entrepreneur, who changes the economy because he innovates. After Schumpeter (1911) introduces the theme of change, Knight (1921) follows up and elaborates on uncertainty (Wubben, 1993).

Knight's second argument illuminates the organic metaphor by investigating the assumptions of perfect competition in classical economics. His account of classical economics comes close to Alfred Marshall's Principles of Economics (1890); especially Knight's assumptions about knowledge of the future and its consequences resemble Marshall's (Knight, 2009/1921, p. 25). Perfect competition assumes omniscience, perfect information, which guides the competitors to a 
profitless equilibrium. But in practice, the assumption of perfect information does not appear to hold, because companies make profits:

The primary attribute of competition, universally recognized and evident at a glance, is the 'tendency' to eliminate profit or loss, and bring the value of economic goods to equality with their cost. [....]. But in actual society, cost and value only 'tend' to equality; it is only by an occasional accident that they are precisely equal in fact; they are usually separated by a margin of 'profit', positive or negative (Knight, 2009/1921, p. 8).

Knight claims that companies make profits (and losses) because of uncertainty. Though change creates uncertainty, change would not cause a problem for the theory of perfect competition if it could be predicted: "Hence it is our imperfect knowledge of the future, a consequence of change, not change as such, which is crucial for the understanding of our problem (ibid, p. 101)." In short, omniscience is a sufficient condition for the absence of profit, yet profit occurs in practice, therefore Knight can refute the assumption of omniscience.

Knight's argument that uncertainty leads to profits, relates directly to investment theory. My view is that omniscience would turn stocks into risk-free bonds. A stock can, like a bond, be valued as the sum of its discounted future cash flow. The cash flow to the owner of a stock consists of the dividends, the parts of the profit that return to the stockholder. Yet, perfect competition eliminates profit. Without profit, a dividend merely equals a return at the risk-free interest rate. A dividend under omniscience bears no risk, because in a world of perfect information pooling insures risk. Thus, in classical economics, stocks should behave like perpetual risk-free bonds, and deliver the risk-free rate of return. Analogously, perfect monopolies and oligopolies would imply predictable profits, which would be priced as risk-free bonds as well, because of risk-free arbitrage. In practice, listed companies make profits because of uncertainty; otherwise stocks would become risk-free bonds. To summarize: transposing Knight's thought experiment of 
classical deterministic price theory to stochastical investment theory, leads to absurdity: omniscience and the phenomenon of stocks exclude each other: profits and stocks exist because of uncertainty.

\section{Keynes}

Knight and Keynes differ in their starting point of arguing against predictability in economics: Knight begins with the assumption of uncertainty and shows that profits cannot be made without uncertainty, while Keynes invents an alternative economic theory which involves uncertainty. Moreover, Keynes invents as well a theory about investing. Uncertainty implies for Keynes a different understanding of economics and human behaviour. He criticizes classical economics, in which uncertainty reduces to risk, and ethics to utility calculations:

The calculus of probability, though mention of it was kept in the background, was supposed to be capable of reducing uncertainty to the same calculable status as that of certainty itself; just as in the Benthamite calculus of pains and pleasures or of advantage and disadvantage, by which the Benthamite philosophy assumed men to be influenced in their general ethical behaviour. [....]. Thus the fact that our knowledge of the future is fluctuating, vague and uncertain, renders wealth a peculiarly unsuitable subject for the methods of the classical economic theory (Keynes, 1978/1937, pp. 112-113).

While Post Keynesians stress that Keynes (1937) upgrades uncertainty to the core of his economics, New Keynesians incorporate Keynes's economics into the mainstream, and leave uncertainty out (Skidelsky, 2009a). In the dissertation, I will opt for the Post Keynesian interpretation of Keynes, because they try to stay close to his ideas about uncertainty. For Keynes uncertainty implies that the government should interfere in the market, because markets are instable and not always self-correcting. Instability and a lack of self-correction imply that a deep recession is not exceptional, but a feature of the market (Skidelsky, 2009a, p. xvii). One cause for instability is that negative economic conditions undermine animal spirits, naïve optimism, which underpin a downturn. Whereas Knight stresses that classical economics excludes profit, Keynes claims that classical economics 
excludes the influence of money, interest rates and financial markets. Please see Chapter 2 for a discussion of Keynes's ideas about his explicit theory of financial markets under uncertainty, which combines the psychology, reasoning, and consequences of human interacting.

\section{Von Mises}

Uncertainty usually has a negative connotation in economics (Wubben, 1993). But the Austrian School of economics takes a positive stance towards uncertainty, and makes it central to its theory. As a consequence, the School denies the aspiration of prediction in the social sciences. In his opus magnum Human Action (1949), Von Mises opposes mechanical causality and, instead, proposes teleological causality, because mechanical causality cannot explain human, intent, behaviour: only teleological causality can. Further, not merely one person acts intently: many people interact with each other. Therefore, human action is a sufficient condition for uncertainty in economics:

The uncertainty of the future is already implied in the very notion of action. That man acts and that the future is uncertain are by no means two independent matters. They are only two different modes of establishing one thing (Von Mises, 2012/1949, p. 105).

Another argument for uncertainty in economics is that no reasonable person would ask the same sort of certainty from the natural sciences:

Natural science does not render the future predictable. It makes it possible to foretell the results to be obtained by definite actions. But it leaves unpredictable two spheres: that of insufficiently known natural phenomena and that of human acts of choice. Our ignorance with regard to these two spheres taints all human actions with uncertainty (ibid, p. 105).

Von Mises's second argument compels because it reduces the scope of what is predictable: though natural sciences can predict specific parts of the world ceteris paribus, it cannot predict how the world looks like in the future. So why expect 
economics to predict the future economy, knowing that intent human choosing is teleological and interactive?

\section{McCloskey}

McCloskey (1990) is a contemporary critic of predictability in economics. She regards economic forecasting as a narrative of the claim to economic expertise. The narrative originates out of the society's expectation that economists can perform the magical task of forecasting. But such easy and profitable forms of predicting do not exist, just as there is no 20 euro bill on the pavement in the neighbourhood of your house: easy opportunities of that kind would just be seized, and therefore do not exist (McCloskey, 1994, pp. 72-73). She puts the forecasting abilities of economists to the test by asking the American question: "If You're So Smart Why Ain't You Rich? (1990)." The answer to the question is one of common sense: economists earn a living by 'selling' their predictions. To earn a living by actually trying to make a profit from putting the predictions to the test in the markets is difficult. Otherwise forecasters would be rich by exploiting their forecasts. Her criticism of predictability is aimed at one dimensional predictions such as the expected return of an investment or the expected level of an economic indicator, but is applicable to stochastical predictability as well. Exploiting both types of predictions would assume time and capital available.

\section{Taleb}

Taleb (2007) is a popular contemporary opponent of predictability in financial markets. He rejects the statistical fundaments of the CAPM, in which investment returns are supposedly stationary and normally distributed. He claims that investment returns have 'extreme' instead of normal distributions, which leads to radical uncertainty. He advocates Mandelbrot's (2004 with Hudson) fractal theory to describe uncertainty, though a fractal distribution could not predict either. Unlike the normal distribution, a fractal distribution allows for exceptional events with a low probability. 
Taleb's key point is that black swans determine the risk of investment portfolios by the changes they provoke. A black swan for Taleb is an event with the features: rare, very influential, and unpredictable. A black swan is Popper's exemplar on the fallibility of induction: only one black swan had to be found to falsify the statement that 'all swans are white'. The black swan has become a popular expression in talking about the unexpected. Yet, Davidson (2010) argues that a black swan is merely a variation on Knightian uncertainty, in which past instances lack to predict the future.

\section{8: Coping with Uncertainty}

In the dissertation I will use Knight's distinction between risk and uncertainty, about which Keynes and Von Mises have comparable accounts. To prevent possible misunderstanding, I will further clarify the meaning of Knightian risk and uncertainty. Uncertainty is the opposite of certainty and risk. Both certainty and risk are species of determinism; certainty determines causally, and risk determines stochastically. The arguments against predictability persuade that Knightian risk is a by-product of certainty, and not relevant for most of economics including investment theory. Though risk does not apply to economics and investment theory, irreducible uncertainty neither does. Under irreducible uncertainty, predictability of specific economic phenomena would be impossible. Irreducible uncertainty would as well deny predictability on more general, non-profitable levels. But few would probably argue against the rationale of the general tendencies of price theory, or against those of diversification and some level of informational efficiency in investment theory.

Because uncertainty is ambiguous, Knight, Keynes, and Von Mises unsurprisingly stress different topics in their treatment of coping with uncertainty. In the contemporary literature, McCloskey and Taleb emphasize on their part new topics, 
and reflect on the scientific practice which had arisen after, and despite of, the seminal contributions of Knight, Keynes, and Von Mises.

\section{Knight}

Knight calls an opinion about uncertainty 'judgment' or 'intuition'. He typifies the epistemological state of uncertainty, which 'lies' in between risk and irreducible uncertainty, as 'partial knowledge':

The essence of the situation is action according to opinion, of greater or less foundation and value, neither entire ignorance nor complete and perfect information, but partial knowledge (Knight, 2009/1921, p. 102).

Partial knowledge underlies an estimate and includes that the estimate can be wrong. How wrong an estimate is, is uncertain, or at the most, roughly assessable:

We know that estimates or judgments are 'liable' to err. Sometimes a rough determination of the magnitude of this 'liability' is possible, but more generally it is not (ibid, p. 116).

Knight does not imply by an estimate or judgment that a probability distribution actually is being made. In fact, the ability to form a judgment by intuition is what counts in business (ibid, p. 118). In practical situations an estimate seems to be reduced to the expected mean of the probability distribution or a qualitative prediction, accompanied by some confidence level, the probability of being right:

Yet it is true, and the fact can hardly be overemphasized, that a judgment of probability is actually made in such cases. [....]. The 'degree' of certainty or of confidence felt in the conclusion after it is reached cannot be ignored, for it is of the greatest practical significance. The action which follows upon an opinion depends as much upon the amount of confidence in that opinion as it does upon the favorableness of the opinion itself (ibid, p. 117).

Knight observes that in the phase after the decision, the estimate 'becomes' certain because earlier doubts are being ignored: 
To be sure, after the decision is made he will be likely to sum all up in a certain degree of confidence that a certain outcome will be realized, and in practice may go farther and assume that the outcome itself is a certainty (ibid, p. 117).

For Knight, risk equals the measurable, and uncertainty the non-measurable. He distinguishes between objective and subjective probabilities along the same lines:

We can also employ the terms 'objective' and 'subjective' probability to designate the risk and uncertainty respectively, as these expressions are already in general use with a signification akin to that proposed (ibid, p. 121).

He explains the assumptions and consequences of subjective probabilities at length, though he does not seem to oppose the use of subjective probability as such. Unlike Knight, Keynes and Von Mises do oppose subjective probability explicitly.

Knight relates uncertainty, imperfection of knowledge, to economic phenomena and the appropriate behaviour towards them:

The task $[\ldots]$ is to inquire more fully into the meaning of this assumption [of practical omniscience]. [.... . On the basis of the insight thus gained, it will be possible to illuminate that large group of economic phenomena which are connected with the imperfection of knowledge (ibid, p. 101).

Under perfect knowledge economic behaviour consolidates cases. But how to decide upon a single case? Indeed, conduct for an individual instance is the same for risk and uncertainty: “[...] when an individual instance only is at issue, there is no difference for conduct between a measurable risk and an unmeasurable uncertainty (ibid, p. 121)." He adds, however, that in the case of risk, insurance overcomes the 'uncertainty' of a onetime trial. Insurance results in the same risk and cost as a pool does. So, economic behaviour towards risk, pools internally or externally by insurance. 
Pooling also handles uncertainty, and results generally in a lower, though not measurable, uncertainty:

And even the third type, true uncertainties, shows some tendency toward regularity when grouped on the basis of nearly any similarity or common element (ibid, p. 124).

Knight characterizes probability estimates about uncertain phenomena as rendering "the greatest logical difficulties of all (ibid, p. 116)". My view is that Knight means that uncertainty is more difficult to understand intellectually than risk. The law of indifference explains risk, because specific causes are not determinable. His account of uncertainty seems to imply that causes could be determinable, but that representative cases lack. Thus, uncertainty can be handled by a probability distribution, by a statistical relation to some cause(s), or by a judgment. Mathematical statistics applies both to probability distributions and regressive statistical relations. Of course, representative cases and regressive predictive relations can only work if they are stationary for some future period.

\section{Keynes}

As Markowitz (1959, p. 257) notes in his seminal work on portfolio theory, Keynes opposes subjective probabilities. Keynes does not believe that the availability of past data solves the problem of subjective probabilities. His approach of logical probability excludes subjective probability: "It [logical probability] is not, that is to say, subject to human caprice. A proposition is not probable because we think it so (2008/1921, p. 4, my insertion)." Logical probability relates probability to evidence, not to psychological reasons.

Keynes $(1936,1937)$ investigates at length the psychological side of subjective probability in the form of expectations, and explains that the present situation dominates expectations if no information about the future is available. He derives how decision makers use the assumptions of stability and correct valuation, 
because they act according to the convention that the future circumstances resemble the present ones, and that the current price correctly reflects the knowledge of the market:

In practice we have tacitly agreed, as a rule, to fall back on what is, in truth, a convention. The essence of this convention - though it does not, of course, work out quite so simply - lies in assuming that the existing state of affairs will continue indefinitely, except in so far as we have specific reasons to expect a change. [....].

We are assuming, in effect, that the existing market valuation, however arrived at, is uniquely correct in relation to our existing knowledge of the facts which will influence the yield of the investment, and that it will only change in proportion to changes in this knowledge; though, philosophically speaking, it cannot be uniquely correct, since our existing knowledge does not provide a sufficient basis for a calculated mathematical expectation (Keynes, 1997/1936, p. 152).

Like Knight, Keynes relates subjective probability to the confidence in a forecast:

It [the state of long-term expectation] also depends on the confidence with which we make this forecast—on how highly we rate the likelihood of our best forecast turning out quite wrong (ibid, p. 148, his italics, my insertion).

Keynes (1936) has a number of suggestions how to decide under Knightian partial knowledge, or degrees of knowledge in Keynes's (1921) terms. He is sober about applying probability to economic phenomena. But what does his account of subjective probabilities mean for his economics? His view is that economics is a moral science, which deals with introspection, values, motives, expectations, and psychological uncertainties. For Keynes, it is rational in economics to form expectations, and make decisions, by using conventions, stories, rules of thumb, habits and traditions.

Another way of tackling uncertainty is to find causal evidence in past data to make the uncertainty partly predictable. Yet, Keynes was a known critic of econometrics, as for example witnessed by his discussion with Jan Tinbergen 
(Keynes, 1939). For the reason of scope, I will merely summarize his critique on econometrics by the account of Skidelsky (2009a, pp. 88-89). His critique is that econometrics wrongfully uses the stability assumption and that its 'free format' modelling expects too much from the data. According to Keynes, one should not use statistics as a default choice: statistics in regressions only make sense for simple and less abstract relations.

\section{Von Mises}

Like Keynes, Von Mises opposes the use of the probability calculus for subjective probability. In attacking the culprit of the theory of subjective probability, he refutes the metaphor that case probability, Knightian uncertainty, is a lottery. The metaphor is flawed, because a tertium comparationis, a common element between case probability and a lottery, lacks:

It is usual to search for the underlying tertium comparationis [at analysing metaphors]. But even this is not permissible with regard to the metaphor [of case probability] we are dealing with. For the comparison is based on a conception which is in itself faulty in the very frame of the calculus of probability, namely the gambler's fallacy (2012/1949, pp. 114-115, his italics, my insertions).

Von Mises refers to the gamblers fallacy to mark the faulty reasoning. The gambler's fallacy means for short that a decision maker expects a representation of the probabilities in the short run, assuming that the probability outcome depends on the recent past. For example, a roulette player expects that the next run will be red, because the last 10 runs were black. The tertium comparationis, the common element between case probability and a lottery is missing, because it is based on a fallacy: the estimate of Roosevelt winning the elections and winning is in a lottery, are both one time trials, which cannot be 'corrected' by a large number of series of the same event later on.

Von Mises (2012/1949, p. 107) stresses that the mathematics of probability is a minor issue compared to that of inference, or induction. Case probability for 
events in the natural sciences such as medicine, does not predict, because frequency does not predict for unique cases:

\begin{abstract}
All such predictions about external events [a doctor's chances for full recovery], i.e., events in the field of the natural sciences, are of this character. They are in fact not forecasts about the issue of the case in question, but statements about the frequency of the various possible outcomes. They are based either on statistical information or simply on the rough estimate of the frequency derived from nonstatistical experience (ibid, p. 110, my insertion).
\end{abstract}

To put it differently, to Von Mises statistics is merely about history, and not about predicting:
Statistics provides numerical information about historical facts, that is, about events that happened at a definite period of time to definite people in a definite area. It deals with the past and not with the future. Like any other past experience, it can occasionally render important services in planning for the future, but it does not say anything that is directly valid for the future. There is no such thing as statistical laws. (Von Mises, 1962, p. 56).

Moreover, multi-causal phenomena imply many interpretations, and can only be interpreted by some theory. The only method for tackling multi-causal phenomena is that of understanding, Verstehen, interpreting, which resembles Knight's partial knowledge and the accompanying judgment, or Keynes's set of answers from rule of thumb to moral evaluations:

The fundamental deficiency implied in every quantitative approach to economic problems consists in the neglect of the fact that there are no constant relations between what are called economic dimensions. [....]. Understanding, by trying to grasp what is going on in the minds of the men concerned, can approach the problem of forecasting future conditions. (Von Mises, 2012/1949, p. 118).

Understanding, or Verstehen, is to be understood as the counterpart of explaining, Erklären, as used in reaction against Comte's positivism. Understanding is about judgment, intentional behaviour, and values (Von Mises, 1962, p. 48). 
Both class and case probability have incompleteness of knowledge in common: both cannot predict in a causal, deterministic way, though the outcome of class probability is predictable as a whole (Von Mises, 1949). The multi-causality in class probability can be reduced to calculable risk and bought off with a premium. But multi-causality in case probability cannot follow the same procedure and has necessarily to be interpreted.

\section{McCloskey}

Concerning inductive inference under uncertainty, McCloskey criticizes, together with Stephen Ziliak (2008), the test of statistical significance in economics and science in general. McCloskey \& Ziliak (2008) did not specifically aim their criticism to (empirical) investment theory. Statistical significance performs a test whether a sample is big enough to confirm a hypothesis, given some likelihood. The likelihood is normally a probability equal or less than $2.5 \%$ that a hypothesis is not accepted by mere chance. Yet, statistical significance as an instrument of induction is inappropriate:

Fit is not the same thing as importance. Statistical significance is not the same thing as scientific finding. $R^{2}, t$-statistics, $p$-value, $F$-test, and all the more sophisticated versions of the them in time series and the most advanced statistics are misleading at best (Ziliak \& McCloskey, 2008, p. xv).

Ziliak \& McCloskey argue against the use of statistical significance, because economics should be about economic significance. Statistical significance does not persuade that a hypothesis is correct or meaningful for most economic research. Relevant is that inductions reflect meaningful relations and show the size of the influence under investigation.

\section{Taleb}

The function of investment models cannot be that they predict: they are merely a description of the presupposed features of the risk and return of some investment 
portfolio (Taleb, 2007). Concerning statistics, the regular concepts of standard deviation, correlation, and regression, make no sense because the supposed normal probability distribution is invalid. A particular point is his statistics regress argument: to establish the type of probability distribution on past data, one has to assume that the probability distribution is of a certain type, which leads to the circularity of data and the type of distribution (2010/2007, p. 269). My view is that Taleb is obviously against subjective probability. He proposes that the central idea of uncertainty is to focus on the consequences of possible outcomes, but not on the unknown probabilities. Because probability does not work, Taleb's investment approach is to combine very conservative investments with very aggressive ones.

\section{9: Implications of Uncertainty for Investment Theory}

Because of the lengthy discussion about uncertainty and probability theory in the preceding paragraphs, I will first summarize the various arguments for, and alternative theories under, uncertainty: Knight argues that the phenomenon of profit excludes a situation of known risk, and that the organic metaphor of change is more appropriate for economics. Keynes relates financial markets explicitly to uncertainty, and invents an investment theory upon rational expectations under uncertainty. Furthermore, he introduces the role of animal spirits in economic

phenomena. Von Mises's argument for uncertainty is first that intent human action features teleological causality, which cannot be predicted, and second that natural sciences do not predict the world as a whole either, so why claim predictability for economics? McCloskey refutes uncertainty by the absence of profitable predictability: if forecasting is easy, economists would get rich by exploiting their knowledge. Taleb's argument for uncertainty is that black swans determine risk and returns of investments; but one cannot predict black swans. Many other arguments could, of course, be brought forward for uncertainty, but the ones presented here suffice to make the case for uncertainty in investing. 
Economic and investing phenomena float in between risk and irreducible uncertainty, and are called 'uncertain'. By definition, the level of uncertainty cannot be certain, otherwise it would be become calculable risk. Certainty and uncertainty express themselves both in numbers and qualitative judgments. Certainty in the form of Knightian risk is numerical, and a deterministic prediction can be a number or a qualitative judgment. Uncertainty can be numerical or judgmental as well. An uncertain judgment has some implicit or explicit likelihood attached. One cannot tell which method of induction is superior: statistics is about an expectation and its dispersion, an opinion or judgment is about a direction with or without a dispersion. For example, an investment manager can decide on investing by statistics, or by the judgment that, say, the economy will do better than expected, which, if correct, could lead to a high return of the stock market. Von Mises, Keynes, and Taleb reject a subjective probability interpretation: statistics is merely a description of the past. Knight does not reject subjective probability, seemingly because it is common practice. My position is that I am not against the use of subjective probabilities, because it is a way of presenting one's expectations. Yet subjective probabilities are an interpretation, not truth, and are not superior to a qualitative judgment.

Keynes's theory of uncertain expectations contrasts the contemporary investment theory of the CAPM. His account of financial markets is distinct from other markets, because financial markets possess instant reflexivity. Markets for goods and services take much longer to react on expectations. His alternative investment theory implies that rational expectations do not lead to predictability, but to instability. The CAPM connects rational expectations to efficient markets, market equilibrium and predictability. Yet, under the condition of uncertainty, Keynes's theory seems more relevant than the CAPM.

In my view Knight's term 'uniqueness' has a twofold connection to uncertainty. The first unique situation is a decision about something new without any 
resemblance to the past. The second unique situation emerges because of unpredictable change. Then, many past instances are available, but are not representative. An example of the second form of uniqueness is a financial market, such as the stock market. My dissertation is about the second form of uniqueness: vast amounts of data are available for a lot of investment decisions, but the future lacks stability. Of course, some 'likeness' with the past can be found, say, for a financial crisis or a recession, which makes an investigation of the past useful. Theory may as well help to find a representative instance: both history and imagination by theory can be helpful. Though, it should be argued why history or imagination are representative and predictive to the case at hand.

A second point of reflection is the meaning of Knight's partial knowledge, or Keynes's degrees of knowledge. In Keynes's terms, uncertainty cannot be expressed as cardinal; the only possible level of distinction is ordinal. Often the ordinality between arguments is unclear. My view is that no intermediate 'risk' state between risk and irreducible uncertainty exists, because if the risk of risk would be known, it can be put into a probability distribution and becomes risk in the form of Knight's statistical probability. Again, risk in the notion of Knight (1921) always means calculable risk. For example, if we know that an investment has an expected return of $6 \%$ and a risk of $20 \%$, and that the risk of the risk is 5 percentage points, than the expected return remains $6 \%$ and the risk becomes $25 \%$. Yet, if we do not know the risk of the risk, we merely have partial knowledge, and thus uncertainty. The distinction between risk and uncertainty is relevant because any statement under uncertainty has no apodictic proof: its arguments need to be judged.

A third reflection on Knight is the effect of diversification under uncertainty. If the future probabilities are known, then randomness is merely a problem of organization. Merely pooling and assessing the cost effectuate the economics. The cases under risk are supposed to be independent, otherwise pooling does not 
deliver certainty. But in portfolio theory investments are dependent. Dependence implies that a residual uncertainty remains. What is the character of residual uncertainty, does a residual uncertainty resemble an unpooled risk? The question reminds of the subjectivist's position in probability theory: for subjectivists, risk and uncertainty mean the same in a one trial decision. Yet, the Ellsberg paradox shows that in one trial decision, risk and uncertainty still differ. Thus, residual uncertainty is of lesser certain status than unpooled risk.

So how does McCloskey's critique of statistical significance apply to investment theory? First, it relates directly to the econometric, empirical part of investment theory, which is outside the scope of the dissertation. Second, an echo of the way of thinking in statistical significance pervades financial risk management. In financial risk management, the so-called value at risk, the absolute or relative money value which can be lost with a small theoretical probability, is set at a $95 \%$ or $99 \%$ probability interval. The value at risk is the minimum loss that can occur with a probability of $2.5 \%$. The method of value at risk assumes that uncertainty can be quantified. The connection with statistical significance testing is the supposed certainty of the procedure. The point here is that the value at risk gets an absolute meaning, while under uncertainty no such guarantee can be given.

Besides her point about the theory and practice of statistical significance testing, McCloskey (1990, 1994, 1996, and 1998) has an answer in coping with uncertainty: in order to better understand the arguments used under uncertainty, a rhetorical analysis is appropriate. Rhetoric has evolved in the realm of uncertainty in decision making and administering justice in Greek antiquity. It is no coincidence that the current dissertation is concerned with the logos, the rational arguments, of unpredictability. Logos is, besides pathos and ethos, one of the three classical ways to make an appeal on an audience. 
Taleb points at the instability of future risks and returns on financial markets with help of the black swan argument. His arguments against the use of the regular statistics and the proposal for Mandelbrot's alternative statistics leave portfolio theory and the CAPM disarmed of mathematical statistics.

\subsection{0: A Thought Experiment with Predictability in Investment Theory}

The current section presents a thought experiment about the assumption of stochastical predictability in investment theory. The experiment puts the statistical outcomes of Markowitz's investment theory, the CAPM, and empirical origin, to the test of risk-free arbitrage, which is the other dominant branch of investment theory, represented by for example option theory. In a model of risk-free arbitrage, a number of transactions in financial, physical, or possibly artificial products, creates a financial product. Risk-free arbitrage implies, in contrast to modern portfolio theory, certainty of outcomes. The thought experiment uses the paradigm of risk-free arbitrage of finance to illustrate that the paradigm of predictability of finance is flawed.

As shown in the current Chapter, stochastical predictability founds the epistemology of predictability in investment theory: Markowitz (1952, 1959), Sharpe (1964), and others, imply that it is reasonable to assume stochastical predictability of risk and return in investment theory. Indeed, statistics and prediction in investment theory look like a tying sale, but they are better to be understood as subjects to be separated.

As explained in the current Chapter, Knight (1921) calls stochastical predictability 'risk': a situation under risk results in predictability when a 'whole' group of cases is available, a situation in economic phenomena which is, however, confined to artificial producers of chance and the actuarial domain. He calls a situation without stochastical predictability 'uncertain'. My conclusion of Knight's distinction 
between risk and uncertainty has been that the application of statistics has no privileged position in comparison to other sorts of arguments under the situation of uncertainty, because the apodictic predictive quality of stochastical predictability lacks. Thus, the application of statistics under uncertainty is merely a form of argument, which can be good or bad like any other argument.

What are the arguments for stocks to achieve a risk premium for achieving a return above the risk free rate? Basically, there are two arguments: one economic argument and one empirical argument. The theoretical economic argument is that investors are risk averse because of diminishing marginal utility, and that therefore extra risk needs to be accompanied by an extra reward. Please note that if investors would be risk neutral, the extra reward would not be demanded. In the case of the stock market, the risk is the systematic market risk, which, by definition, cannot be hedged away, because the market contains all securities. I agree to this economic reasoning. The point of the thought experiment, however, is to clarify that the level of market risk and return is not stochastically predictable, that is, belonging to the category of Knightian risk.

The other argument in favour of a positive risk premium for stocks stems from empirical data. For example, Dimson et al (2014) have shown that in the period of 1900 to 2013 American stocks resulted in an annualized real (corrected for inflation) yield of $6.5 \%$, which is $4.5 \%$ above the return of bonds. An investment in a portfolio of worldwide stocks would have resulted in the period of 1900 to 2013 in an annualized real yield of $5.2 \%$, which is $3.3 \%$ above the return of bonds. The question is whether the historical return of stocks is representative for the future. The rationale of rising stocks could be, say, that if the economy grows, profits of stocks as a subset of the economy could grow as well, and if the valuation of the stock remains constant, then prices of stocks should go up. But things can go wrong as well, the economy might not grow, or the share of the profits of public companies decreases, or the valuation goes structurally down 
because, say, the cult of equities is over. So, the past does not need to resemble the future: therefore the risk premium for stocks is uncertain and not-specifiable.

If stochastical predictability would be a valid assumption in investment theory, then one should be able to buy investment products, say, 'investment model'stocks, with fixed parameters of risk and return above the return on risk-free bonds. Financial institutions, such as investment banks, would engineer such products by the technique of risk-free arbitrage. If the parameters of risk and return would be fixed in the opinion of the provider of the 'investment model'-stocks, the engineering of risk-free arbitrage would be simple:

1. Offer an 'investment model'-stock with a guaranteed specified risk (say $20 \%$ ) and return (say $5 \%$ ),

2. Invest the cash received in real stocks,

3. Pass through the actual return of the real stocks to the buyers of the 'investment model'-stock.

The scheme mentioned above can be extended beyond Markowitz's investment theory and the CAPM; the scheme goes for investment strategies on empirical or other grounds as well. But, the advocates of, say, valuation as a source of prediction, or of a model that offers a $60 \%$ probability of beating the S\&P 500 index, will not offer 'investment theory'-stocks based on their strategies with a specifiable risk and return, because their statistics do not possess the apodictic quality of stochastical prediction either. Of course, all sorts of bigger or smaller inefficiencies emerge in financial markets, which could be exploited. But the inefficiencies are often temporarily, because players on the market catch on to them.

Of course, no one would offer such 'investment model'-stocks as postulated in investment theory, simply because the risk and return of stocks are not fixed, so a 
guarantee on that would be irrational and risky. An investment banker would merely create 'investment model'-stocks, if he could arbitrage without risk. Then, 'investment model'-stocks would become a billion dollar market. In the fictional world of 'investment model'-stocks, investment managers could truly claim reliability of risk and return to their clients, because the models for decision making and risk management, both derived from investment theory, would be epistemologically sound. In the following, I will explore what would be the outcomes of 'investment model'-stocks that the risk-free arbitrage could offer within the realm of stochastical predictability.

\section{An Example of Arbitrage}

First, let us give an elaborated example of the principle of arbitrage. Suppose an American firm needs to pay a bill in Japanese yen in a year, and wants to avoid the currency risk. How could the firm pay the bill in a year without engaging in the currency risk? Well, a bank can offer the firm a forward contract at a fixed rate of exchange, which the bank engineers in a number steps:

1. The bank borrows the dollar amount at the current interest rate in the United States.

2. The bank changes the money borrowed for Japanese yens.

3. The bank puts the amount on a Japanese yen account to receive interest.

4. At the end of the year, the firm delivers the bank the dollar amount, by which the bank pays off the amount borrowed.

5. At the same time, the firm receives the required amount of Japanese yen, which the bank had put on the Japanese yen account.

The procedure explained yields a fixed rate of exchange for a currency hedge. The interest-rate parity theorem relates the interest rates to the forward price of a currency (Ross et al, 1996/1988, p. 832): 


$$
\frac{1+i}{1+i^{*}}=\frac{F(0,1)}{S(0)}
$$

The symbols used mean:

$i=$ the interest rate on a risk-free deposit in the home currency

$i^{*} \quad=$ the interest rate on a risk-free deposit in the foreign currency

$F(0,1)=$ the current forward price of a foreign currency, 0 denotes the start

of the term, 1 denotes the end of the term

$S(0)=$ the spot price of a foreign currency

Theoretically, the price of the currency forward depends only on the difference between the interest rates of the two currencies. If the price of the currency forward deviates from the theoretical price, arbitrage aligns the price with that of the interest-rate parity. The argument of interest-rate parity is not merely theoretically true, it works in practice as well. Interest-rate parity is an example of deterministic, in theory, risk-free arbitrage in investment theory. In practice, arbitrage is not risk-free, because capital is needed for some period and risks occur as well, because of for example the trading in different markets (Shleifer \& Vishny, 1997). Risk-free arbitrage can take a stochastical form as well.

\section{The Thought Experiment}

How could a financial firm create 'investment model'-stocks using risk-free arbitrage? In our thought experiment, the firm can create the risk and return of an 'investment model'-stock by drawing from a normal probability distribution. The parameters of the normal distribution are a yearly expected return of, say, $8 \%$ and a standard deviation, the risk, of, say, 20\%. Microsoft Excel could perform the draws from the normal distribution and deliver the required stochastically predictable risk and return. If only one 'investment model'-stock would be 
marketed, its risk would not be diversifiable and resemble the undiversifiable market risk.

But in risk-free arbitrage a financial firm wishes to arbitrage the risk of drawing of the investment returns from a normal distribution if possible. Because of the random nature of the return of an 'investment model'-stock, the method of arbitrage would have to be similar to that of a casino or an insurer.

The method of arbitrage is to issue a high number of 'investment model'-stocks. Because of the high number of stocks, the issuer attains a calculable and low risk of having to pay more than the expected return. The risk becomes calculable and low, because the drawings of the returns of the 'investment model'-stocks are independent, or to put it differently, the correlation coefficient between the 'investment model'-stocks is zero. In fact, a large number of issued 'investment model'-stocks and the corresponding large number of independent drawings, reduces the issuer's risk of paying more that the promised return close to zero.

To repeat some of the mathematical statistics of diversification of Appendix A of Chapter 2: if the correlation coefficient between the securities is zero, which is the case in the creation of artificial 'investment theory'-stocks, then the risk of the 'investment model'-stock, divided by the square root of the number of issued stocks, decides the portfolio risk of the issuer, in the form of a formula:

$$
\sigma_{p}=\frac{\sigma_{s}}{\sqrt{n}}
$$

The symbols used mean:

$$
\begin{aligned}
& \sigma_{s}=\text { standard deviation of the 'investment model'-stock } \\
& \sigma_{p}=\text { standard deviation of the issuer's portfolio 'p' of 'investment model'- }
\end{aligned}
$$




$$
n=\text { stocks }
$$

The formula of portfolio risk demonstrates that a higher $n$, the number of 'investment model'-stocks, lowers $\sigma_{p}$, the risk of the issuer. Figure 3.1 shows the portfolio risk of the issuer as a function of the number of issues, supposing that the risk of the 'investment model'-stock is $20 \%$ :

\section{Figure 3.1 Risk as function of the number of issues of 'investment model'-} stocks

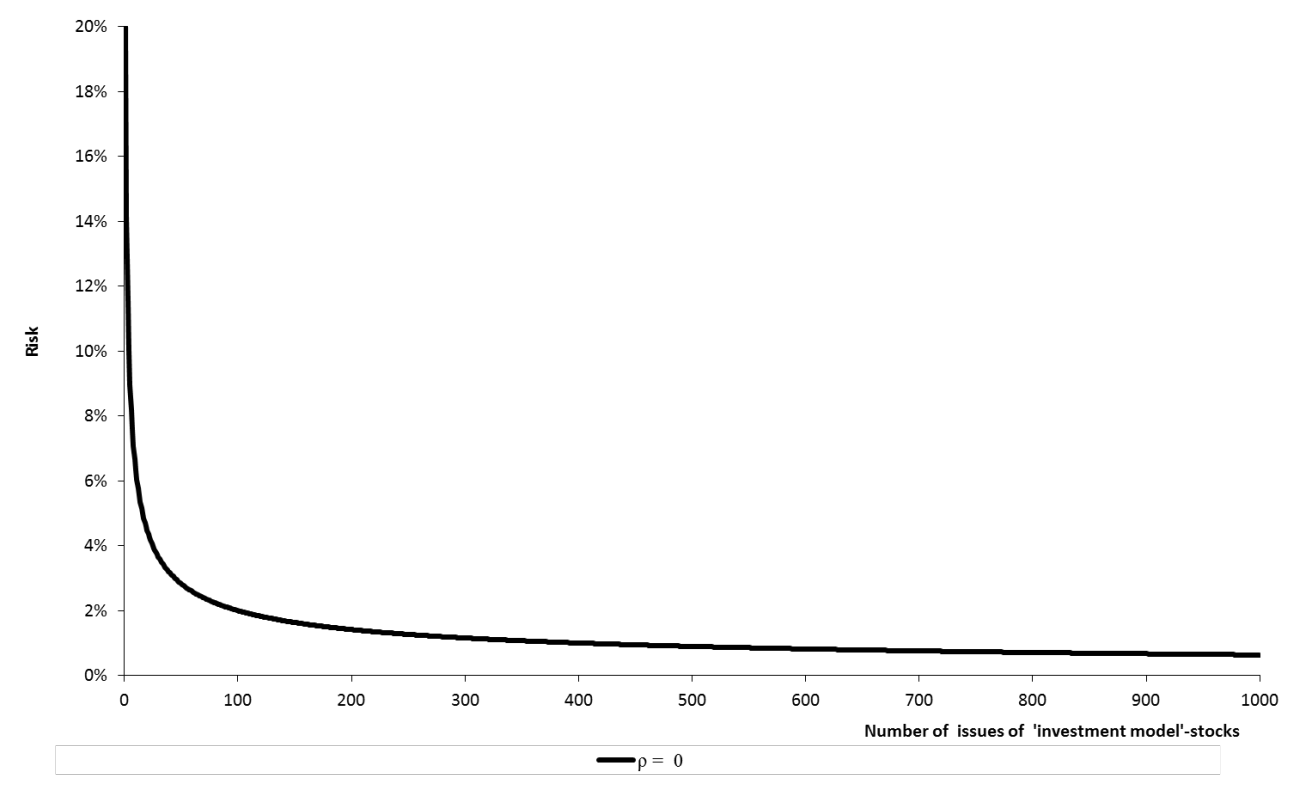

In Figure 3.1, the portfolio risk reduces rapidly as the number of issues enlarges: one 'investment model'-stock has a risk of $20 \%$, the portfolio risk of 1000 'investment model'-stocks drops to $0.6 \%$. Thus, a financial firm can hedge a guaranteed, specified level of volatility if the number of issues is large. If, 
however, the number of issues would be relatively small, the financial firm could offer a lower expected return to reduce the sample risk.

Though the issuer of 'investment model'-stocks could hedge, protect, the risk, 'investment model'-stocks have to deliver a guaranteed return above the risk-free return as well. The issuer has to figure out, how to extract the extra return above the risk-free return of the product by some form of risk-free arbitrage. The issuer receives cash from the buyer of the 'investment model'-stock. With the cash, the issuer could buy real stocks, and use the return of the real stocks to pay the return on the 'investment model'-stocks. Yet, real stocks are no risk-free hedge for 'investment model'-stocks. The cash outflow from the issuer to the buyers of the 'investment model'-stocks will be predictable: it will be, to repeat, close to the level of the specified return. But the return of real stocks will be volatile and uncertain, and will not match the stable specified return of the 'investment model'stocks: so, real stocks do not hedge 'investment model'-stocks risk-free. Moreover, the hedge may turn out worse, because of the Knightian distinction between uncertainty and risk: the returns of real stocks are stochastically unpredictable, uncertain, and the returns of 'investment model'-stocks are stochastically predictable, like Knightian risk.

A high yield bond could be an alternative for real stocks to hedge the return of the 'investment model'-stock. The issuer could buy a high yield bond, and supposing the coupon of the bond equals the yield, use the coupon to pay the specified return on the 'investment model'-stocks. Suppose further that the coupon of the bond equals the specified return, and that the duration of the bond and the 'investment model'-stock have the same length. Then, the yield of the bond hedges the specified return of the 'investment model'-stock. But, uncertainty characterizes a high yield bond: it is incalculable whether the issuer of the bond pays the interests and repays the loan. A high yield bond does not resemble an 'investment model'- 
stock, because the risk and return of the bond belong the Knightian uncertainty and not to Knightian risk, the category in which 'investment model'-stocks reside.

The point of the thought experiment is that a financial firm never can arbitrage, free of risk, an expected return of 'investment model'-stocks above the risk-free return. Instead of a real stock, or, a high yield bond, an 'investment model'-stock resembles roulette or an insurance activity. The most, an 'investment model'-stock resembles roulette, because an 'investment model'-stock issues a probability distribution like roulette does. Instead, an insurer collects a probability distribution. Casinos and insurers carry out their business on the law of large numbers. How do casinos and insurers make a profit? Insurers charge a fee which includes a profit margin and covers for losses, say, the expected loss of an insurance for car damage. In the chance game of roulette, a player can bet, among other things, on numbers up to 36 (Wikipedia, 2013). Casinos tilt the probability distribution of roulette towards an expected return by introducing a zero in the European roulette, or a zero and a double zero in the American roulette. If the turn of the roulette results in zero or double zero the casino wins. In the case of the European roulette with the single zero, the expected return for the casino is $1 / 37$, or $2.7 \%$, without the zero the roulette would result in a zero sum game because the profits and losses of placing bets combined would be zero. An 'investment model'-stock compares to a roulette: yet, the expected pay-out to the holders of 'investment model'-stocks should be positive because of the specified return above the risk-free rate, instead of negative like for the player of roulette, because of the zero.

The moral is that the provider of stochastical predictable risk should receive an expected return, and the buyer should pay, because Knightian risk results in a zero sum game. No serious financial firm would provide 'investment model'-stocks. If a financial firm would market 'investment model'-stocks, the arbitrage strategy would be to borrow at the risk-free rate, to buy a diversified portfolio of 
'investment model'-stocks, and to earn the risk premium above the risk-free rate without taking risk, because, again, the different issues of 'investment theory'stocks are statistically independent.

In the hypothetical case that the market would offer merely one 'investment model'-stock instead of many issues, then too buying the stock would be interesting: risk models would work perfectly and because of the Ellsberg paradox objective risk and return parameters are preferable above subjective ones.

About the claim of economic expertise, McCloskey (1990) says to the economic and investment advisors: "If You Are So Smart, Why Ain't You Rich?". I would like to add another American saying about the narrative of statistical expertise to its advocates: "Put Your Money Where Your Mouth Is!". If the statistical expertise about investment theory is correct than its advocates should be willing to provide the 'investment model'-stocks to the market. Like McCloskey's test, my test is a fair one too, because to market 'investment model'-stocks means to believe in stochastical predictability of stocks. Yet, the advocates of stochastical predictability will reject the test wisely.

In Appendix 3A a mathematical proof formalizes the conclusion of the thought argument that risk-free arbitrage cannot engineer 'investment theory'-stocks with a specified risk and a return above the risk-free return.

\section{Option Theory}

One might object that option theory has not yet been considered as a solution to the engineering problem of 'investment theory'-stocks. Surely options on stocks can create all sorts of pay-offs. Options are available in a great variety of exercise prices and can combine all sorts of exposure to the price of a stock (see Chapter 2 for an explanation of option theory). But, can options create 'investment theory'stocks? 
Despite their flexibility, options cannot create an 'investment model'-stock. To explain why, I focus on the put-call parity as an instrument of risk-free arbitrage. The formula of put-call parity (based on Hull, 1997) looks like:

$$
\begin{gathered}
\text { Spotprice }(\text { Stock })=\operatorname{Value}(\text { Call })- \\
\text { Value }(\text { Put })+\text { Present value }(\text { Zero Coupon Bond })
\end{gathered}
$$

In the formula the call and the put have the same exercise price and time to maturity. The zero coupon bond is bought to exactly match the strike price of the options at the end of the duration. The put-call parity does not need a formula to price the option, or for that matter assumptions about the level of risk or return, though pricing formulas for options need to fit in the call-put parity. Abstracting from the zero coupon bond, the plain intuition behind the parity is that the pay-off of a stock equals the pay-off of a long call, the right to buy the stocks against a strike price, and a short put, the duty to deliver the stocks, against the same strike price, as expressed in Figure 3.2: 
Figure 3.2: Put-call parity, a long call and a short put equal the pay-off of a stock

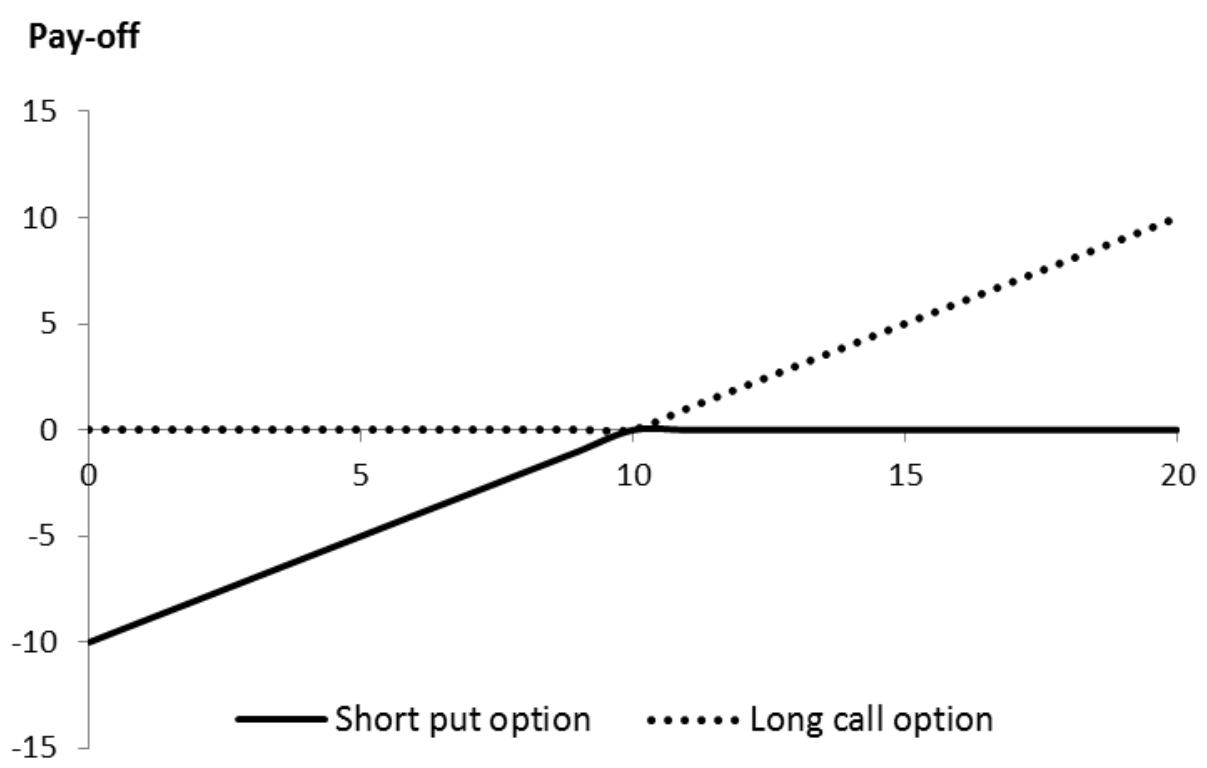

The put-call parity lets a short put and a long call create a normal stock, but no 'investment theory'-stock. To create an 'investment theory'-stock, the levels of risk and return have to be specific and remain stable. Options are bought against a specified level of risk, but their value depends on the price of the real stock, which has no fixed risk and return level. The dependence on the real value of the stock means that the realized risk is uncertain and that no case of stochastical predictability is reached. The only way options could arbitrage 'investment theory'-stocks risk-free would be if stocks would have specified levels of risk and return. 


\subsection{1: Closing Remarks about the Thought Experiment}

Suppose the reader thinks I have made a mistake in my reasoning, and that, after all, risk and return of investments are specifiable. Then, 'investment model'-stocks could easily be made because real stocks would provide the hedge. Merely passing through the return would do the job of providing for 'investment theory'-stocks. Yet, to know risk and return in advance and being able to provide 'investment theory'-stocks with a fixed, specifiable risk and return, mean the same thing: the fact that financial firms cannot deliver 'investment model'-stocks with a specifiable risk and return means that risk and return are uncertain.

I hope to have persuaded that stochastical predictability in investment theory is a false assumption. The thought experiment which shows the impossibility of riskfree arbitrage of 'investment theory'-stocks seems fair, because of the analogy with the artificial device of a currency hedge with the interest rate parity. Only the certainty of interest rate parity, gives a bank the incentive to offer the currency hedge. But with 'investment theory'-stocks such a certainty does not exist.

"So what? It is obvious!" some might reply. Well, for the ones who are not convinced of unpredictability, the thought experiment could make them doubt. It is essential that the use of statistics does not fool one into the idea of predictability. And for the ones who regard the thought experiment as trivial, it is time to make a step forward in using and interpreting investment theory. Then, we agree that the function of the models of investment theory is not prediction. The investment model enables the investors to 'manage' the future with historical and theoretical insights. So, investment theory can only support decisions. Indeed, if statistics merely supports, it should compete with other theories in handling uncertainty: investment theory would be a part of the approach, not the approach. 
Besides uncertainty, investment theory results in certainties as well as represented by the parities. Risk-free arbitrage in the form of interest-rate parity, or put-call parity from option theory, are 'accounting' truths:

Schelling and I are claiming that if you examine important economic arguments you will find 9 times out of 10 an accounting identity overlooked by the man in the street or even by the economist in the study (McCloskey, 2000, p. 175).

Arbitrage assumes that ' $\mathrm{A}$ is B', which makes a trade riskless. Yet, risk-free arbitrage is not possible in the case of investment theory of Markowitz, the CAPM, and other currents of empirical investment theory because capital allocation to investment portfolios is about expectations of risk and return. The expectations are never reducible to stochastical predictability. To put it bluntly: logic $(\mathrm{A}=\mathrm{B})$ grounds arbitrage, while subjective beliefs about the future ground portfolio theory. So, the models or metaphors of risk-free arbitrage and capital allocation for investments differ. The difference does not mean that nothing can be said about expected returns of investments. On the contrary, in the absence of predictability, theories about expected returns should compete, and deliver stories beyond the narrative of a positive risk premium of stocks above the risk-free rate. It sounds logical that a positive risk premium should compensate the risk, but if risk and return are unpredictable, how would you know in advance? It is, however, rational constructivism that makes one think that risk ought to be rewarded, since that would be fair. 


\section{Appendix 3A: A Formal Proof of the Thought Experiment}

A mathematical proof formalizes the conclusion of the thought experiment that risk-free arbitrage cannot engineer 'investment theory'-stocks with a specified risk and a return above the risk-free return. Because the risk and return of an 'investment theory'-stock would be similar to that the risk and return of a casino with roulettes, I start with the formula of the expected return of the roulettes, and the clients of the casino:

$0=E($ return roulettes $)+E($ return clients $)$

Or, rearranged:

$E($ return roulettes $)=-E($ return clients $)$

Symbol used means:

$E(x)=$ expected return of $x$

The formula above, shows, that the parity of the zero sum game of a casino, demands that the positive expected return of the roulettes, opposites the negative expected return of the buyers. Other costs and profits are left out to maintain clarity.

The parity of the zero sum game of a casino resembles the parity of 'investment model'-stocks. Now, the formula includes the time value of money, because in the thought argument 'investment model'-stocks replace real stocks in a portfolio. Strictly though, 'investment model'-stocks and chance games like roulette do not need to be placed in time like real stocks to produce probability distributions, because 'investment model'-stocks and roulette are artificial; a computer program 
produces the artificial outcomes in a split second. The formula of the expected return of an 'investment model'-stock of the issuer and its buyers is:

$0=E($ return issuer $)-R_{f}+E($ return buyers $)$

Or rearranged:

$E($ return buyers $)=R_{f}-E($ return issuer $)$

Symbol used:

$R_{f}=$ risk-free rate

The formula shows that the $E$ (return buyers) is $R_{f}$, the risk free rate when the E(return issuer) is assumed to be zero. Any return that the issuer would claim, would lessen the expected return of the buyers. To repeat, no other sources of return from real stocks, high yield bonds, or options, deliver a risk-free arbitrage above the risk-free return, or for that matter a sensible hedge with risk to cope with the return obligation of a 'investment model'-stock. If the issuer wants a risk-free hedge, he has no choice but to put the money from the buyer on a deposit, in order to give the buyer at least the risk-free return.

Finally, the formalization of the thought experiment will now include the stochastical nature of the 'investment model'-stock. The formula of the zero sum of the probability distributions of 'investment model'-stocks of the issuer and its buyers is:

$0=N($ issuer $)-N\left(R_{f}\right)+N($ buyers $)$ 
in which the symbols used mean:

$N($ issuer $)=N\left(\mu, \sigma_{p}\right)=N(0,0)=0$

$N\left(R_{f}\right) \quad=N\left(R_{f}, 0\right)=R_{f}$

$N($ buyers $)=N\left(\mu, \sigma_{p}\right)=\sum_{i=1}^{n} N\left(\mu, \sigma_{s}\right)$

Symbol used means:

$N(\mu, \sigma)=$ normal probability distribution of expected return $\mu$ and standard deviation $\sigma$

In rearranged terms, the formula of the zero sum game of 'investment model'stocks becomes:

$0=0-R_{f}+\sum_{i=1}^{n} N\left(\mu, \sigma_{s}\right)$

or,

$R_{f}=\sum_{i=1}^{n} N\left(\mu, \sigma_{s}\right)$

The $N$ (issuer), the normal distribution of the issuer, results, to repeat, in an expected return of zero and a standard deviation of zero, because, $\sigma_{p}$, the standard deviation of the issuer's portfolio 'p' of 'investment model'-stocks, reduces to zero, at a huge number of issues. The $N\left(R_{f}\right)$, the normal distribution of the risk- 
free rate, has no risk, and reduces to $R_{f}$, the risk-free rate. The $N$ (buyers), the normal distribution of the buyers, adds the individual distributions of buyers of the 'investment model'-stocks. Please note that the buyers experience randomness, because their risk is $\sigma_{s}$, the risk of the individual 'investment model'-stock. To repeat, the randomness of $\sigma_{s}$ accumulates to zero in $\sigma_{p}$, the risk of the issuer's portfolio. The expected return of the buyer, $\mu$, will equal $R_{f}$, the risk-free rate. 


\section{Chapter 4}

\section{Beyond Statistics: A New Rhetoric for Investment Theory}

"One word is worth a thousand pictures (John Kenneth Galbraith, 1977)."

\section{1: Rhetoric}

\section{The Current Rhetoric}

The current rhetoric of investment theory of nowadays equals the original rhetoric in the seminal work of Markowitz $(1952,1959)$. The logos of the rhetoric of investment theory was discussed in the preceding chapters. Chapter 3 has investigated the epistemological beliefs of investment theory. Markowitz's arguments for using statistics in investment theory stem from Savage's theory of maximizing utility by using subjective probabilities. The continuations of Markowitz's portfolio theory, CAPM and empirical investment theory, are founded on the idea of objective probabilities, or to put it otherwise, the frequency theory of probability. The philosophy of probability justifies the beliefs in investment theory. Yet, merely using objective probability theory, does not make probabilities objective in the Knightian sense. In what follows I will, again, couple 'subjective probability' to Knightian 'uncertainty', and 'objective probability' to 'risk'. In Knight's account, objective probabilities mean the same as 'stochastically predictable'. Deciding whether probability is 'subjective' or 'objective' in the Knightian sense is crucial because it changes what can be legitimately expected of 
decision-making. It appears that Markowitz has put investment theory in the category of risk, instead of in the category of uncertainty where it should belong.

In Chapter 3, the views on the use of statistics in economics of Knight, Keynes, Von Mises, McCloskey, and Taleb have been evaluated. To repeat the common denominator of their view: stochastical predictability is a rare case for economic phenomena, so uncertainty or Von Misean 'case probability' is relevant for economics. Further, a probability statement under uncertainty has the same epistemological status as a qualitative judgment or a regression on historical data. Under uncertainty, statements about economic phenomena are not cardinally measurable like in stochastical predictability: one can merely compare ordinally the trustworthiness of the statements under uncertainty. Besides the historical and contemporary arguments against predictability in economics, I have exposed my own thought experiment against predictability in Chapter 3: a rational financial firm would never market a synthetic stock with a specified risk and return like investment theory assumes, because an 'investment theory'-stock cannot be hedged risk-free.

The arguments against predictability entail that estimated probabilities are subjective in the Knightian sense, so what does that mean for decision making? As shown in Chapter 3, the gap between the subjective and the true probabilities cannot be bridged; merely making larger allowances for risk would not be enough to create stochastical predictability. Using subjective beliefs in the Knightian sense in practicing investment theory gives us no clue to the probability as such that the probability beliefs for the investment decision are right. Moreover, using subjective probabilities in investment theory leads to the same decisions as using objective probabilities. So, subjective is used as being objective, which is dangerous because decision making on the basis of subjective probabilities should not be done by statistics only: under condition of uncertainty statistics should merely be used to explicit the intuition and to help making judgments. 
If science delivers trustworthy outcomes, then science should dominate the input of a decision (Collins \& Evans, 2007). But what to do when one cannot trust the scientific outcomes like in investment theory? To mind comes looking for alternative theories. Yet, no scientific theory can overcome the uncertainty of risk and return of investments. Then, should we abandon investment theory? Probably not. The use of expert knowledge remains preferable, because expert knowledge is, despite its flaws, a better alternative than uninformed knowledge as input into a decision (Collins \& Evans, 2007). I agree that a decision without investment theory is worse than a decision with the theory, though we must propose a new rhetoric for investment theory to lower the importance of statistics, and to promote the use of the practical reason, instead of the theoretical reason. In the current Chapter material from Pistorius (2014) is used.

\section{Plato on Rhetoric}

Let us now explain what rhetoric is about. The ancient tradition of rhetoric as a discipline starts in the Greek antiquity. My concern here with ancient rhetoric is practical, and confined to what is relevant to the dissertation. Thus, although rhetoric in the antiquity was mainly concerned with the spoken word, for the dissertation merely the rhetorical specialities of writing are relevant. Note also that ancient rhetoric is analysed here for the purpose of contemporary appropriation. Contemporary appropriation entails that anachronisms are not problematic, as they would be in a historical approach (Worthington, 2007). Another difficulty is that competing accounts of the classical discipline of rhetoric differ about how to compose and analyse (Gunderson, 2009). Of the competing accounts I will focus on the common elements.

In Greece in the fourth century BC, the theorization of rhetoric matured. At that time Plato seemingly opposed rhetoric, while Aristotle favoured it. Plato was opposed because of the supposed contrast between rhetoric and philosophy: 
rhetoric is not about correctness, but merely about persuasiveness. His negative opinion pervades the customary account of rhetoric today. But was Plato, as for example Vickers (1988) claims, merely an enemy of rhetoric? In the Gorgias, Plato (1925) indeed attacks rhetoric as used in the Athenian democracy because rhetoric capitalizes on the ignorance of its audience, and is not used for deliberation. Thus, he considers rhetoric not as an art, because a genuine art is aimed at our good, such as medicine or gymnastic. Rhetoric is like cookery, a mere flattery, since it just aims at pleasure. Cookery makes food taste better, but it is not, like medicine, aimed at preserving or bettering someone's health. According to Plato, rhetoric is a device for persuasion to unethical purposes and does not aim at the truth, which can only be found by philosophy. Philosophers, being persons who wish to argue carefully, are inclined to reject rhetoric as characterized by Plato (Griswold, 2012). But there is more to rhetoric than what Plato (1925) allows in the Gorgias.

Though Plato (1925) denies in the Gorgias that rhetoric is an art, in the Pheadrus (1914) he suggests that it could become one. With the sophists he agrees that the purpose of rhetoric is persuasiveness. Persuasion ought to move the soul towards the good, so the rhetorician should have knowledge about the soul in order to persuade. Yet, rhetoric becomes an art if rhetoric and dialectic are combined, because rhetoric and dialectic together avert the risk of the improper use of rhetoric. Dialectic is Plato's philosophical method to find truth, which is to be found in the mind, and consists of a discourse by reasoned arguments. By the combination of rhetoric and philosophy, Plato, albeit apparently the enemy of rhetoric, widens the scope of rhetoric beyond political and juridical discourse. Moreover, rhetoric belongs to the philosophical discourse: it is the art of communicating the truth. So, Harvey Yunis disagrees with the usual account of Plato's opinion about rhetoric: 
Any account of Plato's contribution to rhetoric must overcome the traditional view of Plato as the unyielding partisan of philosophy and inveterate opponent of rhetoric in the foundational dispute between the two domains (Yunis in Worthington (ed.), 2010/2007, p. 75).

\section{Aristotle's Art of Rhetoric}

Aristotle arguably did not doubt the merit and the status of rhetoric. He has invented a complete theory of rhetoric. Aristoteles's Art of Rhetoric (2006) is fundamental to studying classical rhetoric (Worthington, 2007). He considers logos, or rational argument, the most important means of persuasion (2006, § I.i.3). For my purposes, a treatment of some basic elements of his theory suffices. His ideas will serve as an illustration of the various parts of the canon of rhetoric.

For making an appeal to an audience, Aristotle (2006) discerns three sources of persuasion: ethos, pathos and logos. Ethos is the moral character as presented by the speaker, it aims at being trustworthy. Trust is evoked in the audience by practical wisdom, virtue and goodwill of the speaker. The second source of persuasiveness is pathos: the speaker has to consider the feelings his words will arouse in the listeners. The third and most important source of persuasion is logos, the arguments about the subject which appeal to the ratio. Logos makes use of induction and deduction. An inductive argument is given by an example, a deductive one is the enthymeme. In economics, a case study can be called an inductive argument, since a case study is an example which can represent other cases as well. The deductive argument of the enthymeme is a syllogism in which the premises are probable, or a shortened syllogism, in which parts are absent to make the listener draw the conclusion (Lanham, 1991). A syllogism is a logical argument, in which the conclusion is inferred from two premises. In the short enthymeme are to be added the conclusion and a premise to get a full enthymeme. The enthymeme occurs a lot in economic reasoning. 
The first element of rhetoric is invention (the Greek and Latin terms are heuresis and inventio respectively). Invention or discovery is about the finding of the arguments. The main source of invention is the commonplace. Commonplaces (topoi, loci communes) are general argumentative patterns, from which specific arguments can be generated for a given conclusion or supposition. An example of a commonplace is (2006, § II.xix.2): "[...] if of two like things the one is possible, so also is the other." The commonplace of 'possibility' reasons by analogy that something which happens in one case can happen in another similar case as well.

The second part of rhetoric, arrangement (taxis, dispositio), discerns the order of a speech, which is introduction, narration, proofs, and peroration:

- The introduction (prooimion, exordium) is the beginning of the speech and is meant to capture the listener's attention,

- The narration (prothesis, narratio) is the statement of the case,

- The proof (pistis, probatio) is a confirmation or thought experiment that the narration is true,

- The peroration (epilogos, peroratio) concludes a speech with e.g. a recapitulation.

Aristotle considers the narration and the proof as the most important parts of the arrangement.

The third part of the canon of rhetoric is style (lexis, elocutio), which means 'how to say things well or elegantly'. Two characteristics of style are clearness and propriety. Clearness improves comprehension and persuasiveness. Propriety of style has an emotional, an ethical and a proportionate element. An emotional relationship to the subject creates sympathy. Ethos is shown by the use of the language appropriate to the situation. Being proportionate to the subject means for example not to make a small matter look big. Style in Aristotle's theory also includes the fourth element of the canon of delivery (hupkrisis, actio) and the fifth 
canon of memory (mnēme, memoria). Delivery and memory are not relevant for written text, so I will not go into them. Another form of style is the trope, which in Greek literally means 'turn'. A trope is a figure like a metaphor, which changes the meaning of one or more words (Lanham, 1991).

\section{Rhetoric in the 20th Century}

In Western history rhetoric played an important role in education. The reemergence of the discipline of rhetoric in the 20th century can be seen as a reaction to logical positivism (Worthington, 2007). A number of $20^{\text {th }}$ century ideas about rhetoric can be illustrated by the work of Chaim Perelman (1958 with OlbrechtsTyteca) and Stephen Toulmin (1958), and by the development of literary criticism. Perelman coined the term 'The New Rhetoric' (1979). His main work is The New Rhetoric: A Treatise on Argumentation (1958). In the 1940's he was attempting to resolve the dilemma of the logic of value judgments. According to logical positivism, value judgments like judicial ones are not scientifically founded. But he could not agree with the positivist conclusion that value judgments are merely a matter of taste. An outcome of his search for a foundation of value judgments was a rediscovery of Aristotelian rhetoric. Besides Perelman, Toulmin (1958) has been influential in rhetorical theory in the 20th century. He also turns away from logical positivism. In The Uses of Argument (1958) he proposes a new argumentation theory based on actual ways of argument such as legal reasoning. His theory investigates the 'partial argument', which is close to Aristotle's enthymeme.

Besides within philosophy, rhetorical analysis developed in literary criticism as well. Literary criticism has, like rhetoric, a long tradition:

A partial list [of literary criticism] in historical order would include rhetorical, philological, Aristotelian, belletristic, hermeneutic, historical, new critical, psychoanalytic, neo-Aristotelian, archetypical, neorhetorical, Marxist, readerresponse, deconstructive, linguistic, feminist, and new historicist criticism (McCloskey, 1998, p. 16, my insertion). 
Literary criticism investigates metaphors, narratives, and other rhetorical elements that writers use to achieve persuasion.

\section{2: The Rhetoric of Economics}

McCloskey's discourse analysis of economics in The Vices of Economists; The Virtues of the Bourgeoisie (1996) will be used here as a frame of reference for further describing the current rhetoric of investment theory, and for inventing a new rhetoric. The Vices of Economists is a rhetorical criticism of the discourse of economics in general. Adopting McCloskey's discourse analysis, investment decisions require two things: an analysis of its rhetoric, and bourgeois virtues for coping with investment decisions. The analysis of rhetoric provides insights into the beliefs, the justifications, and the broader context of investment theory.

First, I will discuss four books that make up her conception of the rhetoric of economics. In the books she analyses the phenomenon of rhetoric as used in economic texts. In the preface of The Rhetoric of Economics (1998) McCloskey refers as key literature to:

1. If You're So Smart: The Narrative of Economic Expertise (1990)

2. Knowledge and Persuasion in Economics (1994)

3. The Vices of Economists: The Virtues of the Bourgeoisie (1996)

4. The Rhetoric of Economics (1998, second edition)

The works seem to constitute the end product of her work about rhetoric. She started publishing about rhetoric with the article The Rhetoric of Economics (1983), out of which the book with the same title originated (1985a), with a revised second edition in 1998. Both the article-version and the first edition of the book attracted 
attention of philosophers of economics because of her critical stance towards the modernist methodology. Modernism is called positivism in philosophical terms.

A comprehensive answer to the criticisms of philosophers of economics came in Knowledge and Persuasion in Economics (1994), in which she elaborates her views about philosophy and philosophy of science. Too, she discusses the differences between rhetoric and philosophy, and the relation between the two reflective approaches. The book If You're So Smart: The Narrative of Economic Expertise (1990), is of special interest for my dissertation, because here she presents economic forecasting as a narrative of economic expertise.

In The Vices of Economists; The Virtues of the Bourgeoisie (1996) she remarks that economics has become a game in the sandbox, in which economists play like little boys. But the sandbox is not like the real world, despite the seriousness of the boys in their play. The book is a rhetorical criticism of the discourse of economics in general. After picturing three vices of current economics, it suggests a future bourgeois moral for the field, that of virtue ethics. It considers prudence not as the only virtue, but as one of the necessary virtues, such as temperance and justice. She elaborates her ideas about virtue ethics in The Bourgeois Virtues, Ethics for an Age of Commerce (2006). Since The Vices of Economists is a rhetorical criticism of the discourse of economics in general, the three vices will now be treated in some depth:

\section{Statistical significance}

The belief that statistical significance replaces scientific significance in testing a hypothesis follows the ideas of Lawrence R. Klein (McCloskey, 1996). As explained in Chapter 3, statistical significance is not sufficient to accept a scientific result, because it has nothing to do with relevance. Moreover, it leads to irrelevant science. What does matter, is the magnitude of a scientific finding. Because the testing of statistical significance is almost obligatory in science, abolishing it is controversial 
(Ziliak \& McCloskey, 2008). Though it does apply to the empirical side of investment theory this will not be elaborated in the dissertation because it is outside its scope. In The Cult of Statistical Significance: How the Standard Error Costs Us Jobs, Justice and Lives Ziliak and McCloskey (2008) explain the compelling case against the use of statistical significance in science.

\section{Blackboard economics}

Given an axiomatic set of assumptions in a model, the existence of some economic outcome can by proved by a calculation or inference on the 'blackboard'. Blackboard economics is considered as science and has been promoted by Paul Samuelson. But the 'Samuelsonian vice' has led mainstream economics to become purely theoretical. Of course, McCloskey (1996, p. 72) agrees that "Theory is good. It is the disciplined imagination of economics." Mathematics also explicates the mechanism of an economic theory (1998). But the problem is that blackboard economics has been overdone.

\section{Social engineering}

The most important vice is the belief that the first two vices can be used for economic policy. The question 'why is social engineering as proposed by Jan Tinbergen a vice?', has a simple answer: 'it does not work'. The arguments for the failure of social engineering are not new:

[...] the Austrian School long ago and the Rational Expectations School during the past quarter century have pointed out that it is ourselves we are trying to engineer. That's the big problem. The reflexivity of economics sets stringent limits on what we can predict and control (McCloskey, 1996, p. 103).

To my opinion the third vice applies arguably to predicting in investment theory. 
McCloskey's discourse analysis also tries to analyse economics as products of a scientific culture. According to McCloskey, the dominant culture of science is modernism, the official methodology for mainstream economics which is based on logical positivism. McCloskey's (1998, p. 148) view is that economics choose modernist methodology, because the argument of philosophy is one of authority. But her point is that philosophy itself has changed its mind, as expressed by for example Michael Polanyi (1958), Paul Feyerabend (1975), and Richard Rorty (1979). Polanyi (1958) argues that the modernist idea that subjective 'observation' is not scientific, is outdated, which he elaborates in his book Personal Knowledge

$[\ldots]$ is to show that complete objectivity as usually attributed to the exact sciences is a delusion and is in fact a false ideal (Polanyi, 1974/1958, p. 18).

Another modernist principle is that science and non-science can be demarcated by methodology. The prescript presupposes the existence of such a methodology. But Feyerabend does not believe in one strict methodology; on the contrary, he recommends an anarchy in methodology as an "excellent medicine for epistemology, and for the philosophy of science (2010/1975, p. 1)." His point is that methodology depends on the character of the scientific problem and that methodology cannot be fixed.

Rorty discusses a more general point about modernism. He regards the problems of modernist methodology as coming from the misleading metaphor that the mind is a mirror of nature (1979). The metaphor implies an external reality independent of our mind. But in the pragmatist philosophy such dichotomies as internal and external, or, objective and subjective, are considered as flawed.

\section{Rhetoric and Philosophy of Economics}

McCloskey does not believe in the official methodology of modernism for mainstream economics, and has found substantial evidence and support from inside philosophy for her anti-modernist position. Notwithstanding, her alternative of 
rhetoric of economics has received critical attention from philosophers of economics. The philosophy of economics and the rhetoric of economics have in common that they reflect on economics. Both aim to achieve a better insight into the working of the science of economics. But how do they relate to each other? Can rhetoric be seen as a branch of philosophy of economics?

According to one of the leading philosophers of economics, Hausman (1981), the philosophy of science broadened its perspective in the 1960s and 1970s from methodologically prescribing how science should work, to empirically describing how science is actually being done. The broader perspective of philosophy of science has also arisen from the loss of trust in logical positivism. Despite Hausman's (1989) interest in eclectic methodology, he is critical of McCloskey's rhetorical approach:

But McCloskey offers little solid argument for employing his favored literary tools, and he has a hard time explaining how his proposed successor to economic methodology is supposed to retain any normative role. And the normative role of methodology is unavoidable; whether methodological rules are garnered from imitation, methodological asides, or systematic methodological treatises, there is no doing economics without some standards or norms. Furthermore, if economics is to make any rational claim to guide policy, these standards or norms cannot be arbitrary (Hausman, 1989, p. 123).

The criticisms of McCloskey's approach by Hausman (1989), Hausman \& McPherson (1988), and Hollis (1985) provide an opportunity for explicating the difference between the primary beliefs of rhetoric and those of philosophy of science. McCloskey (1994) has replied to the individual criticisms and devoted Knowledge and Persuasion in Economics to elaborating her point of view about rhetoric and its relation to philosophy. Hausman \& McPherson (1988) miss the normative element in McCloskey's rhetorical approach. They describe her standards as those held within a discipline or intellectual standards in general. Anything more would be normative methodology, which McCloskey has rejected. 
But they dislike the division between general standards and normative methodology, in their words:

What's left when the formulas and rigid rules are gone is the exercise of informed judgment, guided by broad and evolving principles of assessment, which in turn still rest on implicit or explicit epistemological theories. That's Methodology, too (Hausman \& McPherson, 1988, p. 6).

One might conclude that Hausman's \& McPherson's and McCloskey's points of view have come rather close, apart from the dispute over the existence of implicit or explicit epistemological theories. Yet, Collins \& Evans (2009/2007, p. 39) seem to agree with McCloskey's point of view: in their discussion about the nature of expertise, reflective ability is a generalized skill, a form of expertise applicable to all sorts of sciences and arts, which is different from an epistemological theory.

Martin Hollis (1985), a philosopher of social science, criticizes McCloskey's rejection of modernism in the 1983 article-version of The Rhetoric of Economics. His criticism is that a rejection of modernism should not imply a rejection of traditional epistemology:

For instance, no Cartesian or traditional rationalist would accept any of them. So there can be no general presumption that to reject modernism is to abandon the old search after truth by methods grounded in traditional epistemology (Hollis, 1983, p. 2).

The criticism of Hollis amounts to that of Hausman \& McPherson (1988): his problem is that the rhetorical approach has no epistemology, in other words, no methodology to determine truth.

But according to McCloskey, epistemology itself, the philosophy of knowing, is the root of the problem (1994). What Hollis means by epistemology presupposes the existence of an objective form of truth, while she presupposes merely a 
pragmatic form of truth, like that proposed by Rorty (1979) and his predecessors in pragmatic philosophy. Given her position, it makes sense to distance oneself from strict methodology, just like the anti-methodology standpoint that Feyerabend (1975) proposes. Nevertheless, the methodologists of economics see rhetoric as a part of methodology. But McCloskey has no wish to be part of methodology, because the current philosophy of science cannot deliver a standard to which argumentation can be held.

\section{Anti-philosophy and the Moral of Rhetoric}

Arjo Klamer has explained the dispute between methodologists and rhetoricians as a matter of different perspectives:

Since this linguistic turn the books of conventional methodoloy are gathering dust on my shelves and I had to gather an entire new selection of books for my library. [....]. We not only use different concepts, ask different questions, but we also read a different literature and relate to different texts. [....]. The critics turn out not to have read Aristotle, Perelman, Toulmin, Foucault, Booth, Rorty and so many others who have written about rhetoric and discursive practices. It's no wonder, therefore, that we have such a hard time communicating (Klamer, 2001, p. 73).

How does McCloskey compare rhetoric to philosophy? McCloskey sees positivism as a reaction to German idealism. Logical positivism was meant to end speculation about metaphysics. But not talking about metaphysics does not make it disappear. She sees rhetoric as a replacement for the current philosophy:

We need a new philosophy, or anti-philosophy, to understand economic science as she actually works. The form of anti-philosophy recommended here is the oldest one, 'rhetoric' (McCloskey, 1994, p. xiii).

She has no need of a methodology: "An economic methodology 'based' (that hopeful word) in philosophy [...] is too thin to work (McCloskey, 1994, 86)." Moreover, the conception of economics within macro-economics is plural: the neoclassical school, the Austrians, the Institutionalists, and the Marxist have 
divergent starting points. The starting points are decisive turns within economics and determine the way of looking.

German idealism has now returned in a more mature version, as pragmatism or rhetoric. Rhetoric has the advantage that it is interpretative and introspective about metaphors and stories. Idealism in such a way is based on social discourse. In the end, the philosophy of economics is about morality, an intention which a number of philosophers of science share with her. McCloskey urges a Sprachethik in which the ethical side of a conversation is stressed:

[...] speech morality, the ethics of conversation. [...] Don't lie; pay attention; don't sneer; cooperate; don't shout; let other people talk; be open-minded; explain yourself when asked [...] (McCloskey, 1994, p. 99).

Modernist science does not fulfil conversational demand, also because morality is seen as mere preference (McCloskey, 1994). The discipline of rhetoric, in the spirit of the Roman statesman and author Cato of 'vir bonus, dicendi peritus', 'a good man, skilled in speaking', seems a promising alternative to the philosophy of economics.

\section{3: Metaphors and Stories}

\section{The Rhetorical Tetrad}

McCloskey's interpretation of rhetoric combines the tools of classical rhetoric with those of literary criticism. Literary criticism permits one to bridge the gap between literature and science: "The scientific report is itself a [literary] genre, whose conventions have changed from time to time (1990, p. 30, my insertion)." Her literary approach is adapted to the specific nature of economic literature: "Economics may be like poetry in this or that important respect, but plainly it is not the same (McCloskey, 1994, p. 44)." Central to rhetoric is the art of argument, i.e. 
skilfulness in using reasons to show that something is true or correct (McCloskey, 1990). Rhetoric is used by all economists:

Rhetoric is unavoidable. An economist or historian cannot avoid writing rhetorically since any argument has a rhetoric, a style of argument, taking 'argument' to mean 'any designs on the reader' (McCloskey, 1990, p. 56).

She explains her approach of the rhetoric of economics by the rhetorical tetrad, consisting of four elements: fact, logic, story and metaphor. All these elements help in exploring economics (1994). The key point of the tetrad is that economics must be based on more than fact and logic, on which current economics is centred, due to its modernist background:

The choice to have high standards of logic, low standards of fact, and no explicit standards of metaphor and story is itself a rhetorical one. It depends on the audience of economic scientists (McCloskey, 1990, p. 23).

Thus, economists lack self-awareness about their use of metaphors and stories. To the concept of storytelling a modernist would respond that a real scientist just finds the story, so science is about reality and not 'made up' like in fiction. But by just telling the story like it is reality, the modernist economist avoids the responsibility to examine his perspective. Denying the existence of a perspective means to deny that people, not the external world, make stories. Economists deny the use of the metaphor in the model as well.

A feature of the rhetorical tetrad is that each of its parts limits the possible excesses of the other parts. Metaphors and stories are the two ways of answering the question 'why?'. Metaphors and stories in economics can criticize each other, and co-exist. An allegory integrates metaphor and story. Following the empiricist tradition, a model and a story are to be checked by the facts. So facts constrain a model and a story. Logic also has its role. In the spirit of the rationalist tradition, models and stories are to be investigated by logic for their consistency. Yet, a 
narrow focus on logic and facts can lead to bad science, just like a narrow focus on story and metaphor.

The metaphor is very relevant in economics because the economic models are to be seen as metaphors. The choice of a metaphor in economics reflects a worldview of a school of economics and is a figure of thought, not merely a figure of language. McCloskey proposes as a definition for a metaphor that it brings together "two separate domains into cognitive and emotional relation by using language directly appropriate to the one as a lens for seeing the other (Black, M., 1962, Models and Metaphors, p. 236 cited in McCloskey, 1990, p. 12)." Another definition of a metaphor is:

Changing a word from its literal meaning to one not properly applicable but analogous to it: assertion of identity rather than, as with simile, likeness (Lanham, 1991, p. 100).

Besides using metaphors in the usual sense, the economist uses them in the form of the economic models:

The market for apartments in New York, says the economist, is 'just like' a curve on a blackboard. No one has so far seen a literal demand curve floating in the sky above Manhattan. It's a metaphor (McCloskey, 1990, p. 1).

Metaphors in the form of models work best in economics at making standard predictions, for example how higher interest rates affect the housing prices. A metaphor is not 'true' in the sense that it corresponds with the external world as in the correspondence theory of truth: it is merely useful for a purpose. A problem arises when metaphors are taken literally. Then metaphor becomes identity: instead of one of the possible perspectives the metaphor becomes the only perspective and absolute.

Besides using metaphors, an economist tells stories: 
Plainly and routinely, 90 percent of what economists do is such storytelling. Yet even in the other 10 percent, in the part more obviously dominated by models and metaphors, the economist tells stories (McCloskey, 1990, p. 16).

Indeed, economists explain their models in the more accessible form of a story or a narrative. To find stories in economics is easy:

The actions of an economistic folklore are few: entry, exit, price setting, orders within a firm, purchase, sale, valuation, and a few more. [...]. Economists say over and over again, 'action X is just like action $\mathrm{Y}^{\prime}[\ldots]$ (McCloskey, 1990, p. 24).

The story is a fiction, constrained by, but underdetermined by the facts. Just like metaphors, stories are selective, because they focus on what is important. Storytelling as such is scientific. Storytelling works best at understanding something that already happened (for example, explaining the economic depression of the 1930s), but is used for predictions as well. Economists themselves do not always realise that they are telling stories. Rhetorical criticism sensitizes us to the possibility that a writer, possibly unconsciously, tries to make us look in a particular way. Stories can also be dangerous for the public which takes the economic stories literally.

\section{Metaphors and Stories in Investment Theory}

I now apply McCloskey's rhetorical approach to investment theory to discover the main metaphors and stories in Markowitz's theory, the CAPM, and some of the alternative investment theories and theories of uncertainty. The main metaphor in Markowitz's investment theory is the claim that investment management is mathematical statistics. The application of mathematical statistics assumes calculability and distracts the attention from uncertainty. The metaphor of mathematical statistics makes us see only through the perspective of calculability: it makes us think in terms of probability distributions, correlations, criteria for efficiency, and algorithms as the necessary elements. The second metaphor in 
Markowitz's theory is the machine. The machine metaphor, a well-known form in management theory and indeed in political theory and sociology generally, can be recognized in the language of, and aspiration to efficiency. 'Efficiency' in Markowitz's theory means that an investment portfolio has a maximum return given a level of risk. The machine metaphor implies that risk and return can be optimized by precise calculations, which can and should determine the composition of the investment portfolio.

Both the metaphors of mathematical statistics and the machine underplay the importance of the beliefs about risk and return. Beliefs are viewed as merely input for the theory. Moreover, the beliefs cannot be seen as a problem once the jump to mathematical statistics had been made. The machine metaphor focusses on the process parts of reaching efficiency, such as the efficiency criteria and the algorithms, and beliefs are treated merely as inputs for the calculating machine.

Markowitz's metaphor of mathematical statistics in story form is that applying mathematical statistics is a sensible and sophisticated thing to do: statistics allows you to engineer your investment decisions like an actuary manages an insurance portfolio. It is superior to a less sophisticated quantitative or qualitative approach. A second story is that the theory finds an efficient trade-off in risk and return by running its algorithms. Before Markowitz there was no 'efficient' frontier of risk and return: investment portfolios are to be directed to the efficient frontier.

The dominant metaphor in the CAPM is the equilibrium: the market of investments is in balance because of informational efficiency and rational expectations. In equilibrium, risk and return have become predictable. Equilibrium and predictability are exchangeable. The second important metaphor is the informational efficiency. It is again a machine metaphor, but now concerning the processing of information. Because of informational efficiency the market of 
investments is at any time in balance: new information is immediately being processed into a new equilibrium.

A dominant story that pervades modern investment theory is the idea that running risk should receive a reward. Thus, stocks should earn a premium, aka known as the risk premium, above the risk-free rate, because investors are risk averse. Besides the rational constructivist point of view, historical evidence is used. For example, Jeremy Siegel (1994) analyses that stocks earn a risk premium in the long run of a period of 20 years. To repeat, the rationale of rising stock prices could, say, be that if the economy grows, profits of stocks as a subset of the economy could grow as well, and if the valuation of the stock remains constant, then prices of stocks should go up. But things can go wrong as well, and therefore the past does not need to resemble the future.

Besides informational efficiency in story form, the dominant story in the CAPM is that an optimal portfolio is no longer at the Markowitz efficient frontier of risk and return, but a combination of the risk free return and the market portfolio. The market portfolio consists of all securities, which are correctly priced, so no advantage could be gained by choosing another portfolio. Another important story is that diversifiable risk does not deserve a reward: only the exposure to the undiversifiable risk is rewarded.

The metaphors and stories of current investment theory persuade because financial economists have been trained to accept the ideas of equilibrium and calculability, which belong to mainstream neoclassical economics. But investment theory, especially the CAPM, is to be seen as an ideal type: informational efficiency in combination with rational expectations seems utopian.

Let us now investigate the metaphors of the opponents of predictability in economics and investment theory. Knight proposes the metaphor for economic phenomena to behave like an organism: the organic includes the aspect of change 
of economic phenomena. An organism adapts to new, unforeseen circumstances, while a mechanism adheres to strict rules. Keynes illustrates the functioning of a financial market by the metaphor of a beauty contest. To win the contest, a participant has to predict which face the other contesters will choose. The metaphor of the beauty contest stresses how human interacting works out, and opposes the valuation theory, in the sense that valuation is not fundamental but constructed in the beauty contest. A related metaphor of Keynes summarizes how to act under uncertainty and formulates an efficient market theory without some mechanism of equilibrium: Keynes's efficient market theory leads to the conclusion that financial markets are instable. Von Mises stresses that causes are teleological instead of mechanical: teleological does not mean here 'the striving towards a collective equilibrium', like in the CAPM, but the striving of many individuals for their goals, which results in unpredictability. McCloskey highlights that predicting in economics is magical. Magic persuades people, because they believe the narrative of expertise. Taleb uses the metaphor of the black swan, which is a rare, very influential, and unpredictable event. The metaphor of the black swan stresses the fallibility of induction on which investment theory founds the probability beliefs of its models.

Alternative investment theories have metaphors to their aid as well. I will discuss a number of the metaphors. The ideological criticism of investing equates investing to gambling. By saying investing is gambling the dark side of investing is highlighted, including manipulation and fraud, which as we all know are part of investing. The metaphor of Galbraith, Minsky, Kindleberger and Shiller is that financial markets are like bubbles which can burst. A bubble is reinforced by lending and self-reflexive social mechanisms, but is not grounded in economic facts, so it bursts, sooner or later. Mandelbrot proposes wild instead of mild randomness as the metaphor for financial markets, and therefore that investment theory needs an alternative statistical theory. 
In the next Chapter, the metaphor of investing as culture is explored, and other metaphors could be interesting as well, such as that investing is a power relation analogous to the agency theory of Jensen \& Meckling (1976), which will be treated in the next Chapter in one the cases of innovative investment practice.

The metaphors of the uncertainty economics and alternative investment theory highlight other aspects of investing than the current investment theory does, and makes us see its weak spots.

\section{4: Virtues}

Because the theoretical reason of investment theory results in weak outcomes, the practical reason should dominate in decision making. Practical reason is about introspection, values, and ethics. McCloskey (2006) favours bourgeois virtues as a balanced system of ethics. McCloskey investigates the role of virtues of our capitalist, bourgeois society in the tradition of Adam Smith (1790). In Greece of the antiquity, Aristotle (1908) has laid the foundations of virtue ethics in Nicomachean Ethics. The seven virtues that McCloskey distinguishes, consist of four pagan virtues: courage, temperance, justice, and prudence, and three Christian virtues: faith, hope, and love. A virtue does not tell you what to do, but is about character and fits to all kinds of situations:

A 'virtue' is a habit of the heart, a stable disposition, a settled state of character, a durable, educated characteristic of someone to exercise her will to be good (McCloskey, 2006, p. 64).

Virtue ethics contrasts modernist ethics because virtue ethics is grounded in human nature and tradition, instead of in subjective utility or abstract reasoning (Audi, 1995). Subjective utility founds the ethics of economics. In the virtue system of McCloskey, thinking in terms of utility is about the virtue of prudence, which is the 
main virtue of neoclassical economics. By prudence or phronesis she means practical wisdom, know-how, or common sense (2006, p. 253).

But economics should be more than prudence, which also represents ethics in investment theory. Not all virtues seem applicable to investing, for example faith and hope seem less relevant for such a secular activity. Let us illustrate what other virtues can mean for using investment theory. Investing is often done with other people's money. The virtue of temperance is about individual balance: temperance demands self-control and epistemological humbleness of the people who decide about investing or research on investment theory. Temperance means that investment professionals and researchers should not gamble: with leverage it is easy to gamble in investing, but it takes merely a financial crisis to put investment strategies to a stress test, resulting in the destruction of wealth. Justice is about social balance and demands, like the virtue of love, to care for the owners of the managed investments. The clients often do not have the knowledge about investing that the investment professionals possess: that makes the clients vulnerable. But it cannot be fair that the epistemological uncertainty of investing is transferred to the clients. So, investment managers should communicate about the uncertainty, be humble, and take care as good as possible of the client's money.

Following McCloskey's discourse analysis of economics, the new rhetoric for investment theory requires qualitative judgment based on intellectual honesty, insight in its rhetoric, and bourgeois ethics. Because investing takes place under Knightian uncertainty, statistics are supportive rather than dominant in decision making. And alternative investment as treated in Chapter 2 should be a part of the discussion as well. So, investment theory should become more a moral science and less an engineering one: it is about judgment, combining virtues with historical and theoretical insights. Of course, no fixed methodology should exist in the domain of the practical reason as well, so virtues are interesting possibilities beyond the onesided solutions of prudence-only or other monolithic modern ethical theories. In 
the next Chapter the culture of investing and innovative cases from practice will be central. Culture is both a natural extension of rhetoric and uncertainty. 


\section{Chapter 5}

\section{The Culture of Investing}

"When I hear the word culture I reach for my ethics (Tweet Deirdre McCloskey, 5 September 2014)."

\section{1: Culture, Economics and Finance}

\section{The Relevance of Culture}

Economics and finance ignore culture. The education of economists leaves culture out, neither is culture an explicit part of the finance education; no wonder, because economics and finance are modelled after physics, which indeed does not need culture as a source of explanation. In line with the modelling of economics after physics, the philosophical context of modernism has caused economics and finance to shun the human part of reality, such as values, ethics, and culture:

\footnotetext{
By 'modernism' we mean the claim to practise science as we understood the term in secondary school. Extract from life if you can what is historical, value-laden, judgmental, ethical, cultural, tacit, skilful, smooth, curved -'all things counter, original, spare, strange'; and what will be left is modernism. It consists of the simpler parts of science and scholarship. Modernism [...]has urged us to leave strictly to the side the matters of moral force and human meaning (Klamer \& McCloskey, 1992, p. 156).
}

By relating culture to economics, Klamer poses a question in the Weberian tradition of distinguishing between procedural and substantive rationality (Klamer, 2014, p. 29). Economics is about procedural rationality, culture is about substantive rationality. Both substantive rationality and culture centre at values, or, the answer to the question 'What is important?' Sociology, anthropology, and philosophy include the perspective of culture. The inclusion of culture in economics could yield a richer economics as well. For example in decision 
making, economics and finance rely on the calculations of rational choice, though the notion of culture would beg for the alternative of phronesis, that is the practical wisdom to do the right thing:

Economists embrace the idea [of means to an end, measured in utility] because it enables them the modelling of decisions in the form of mathematical equations. The modelling gives the idea the aura of 'science'. The idea of phronesis makes us realize that too much is involved in doing the right thing, that calculation is therefore nigh impossible, and modelling quite hopeless (Klamer, 2014, p. 27, my insertion).

In addition to values, Klamer's cultural approach of economics emphasizes conversation as a way to interpret culture. The point of using conversation to understand investment theory can be illustrated by the role of talk in the stock market:

The chatter in the stock market (that ideal of a marketplace) is another example of persuasion in the economy. Portfolio managers talk full-time to decide on buying or selling. Stockbrokers talk to clients and to each other. Technical elves spend their days researching the thoughts the brokers ought to have. Journalists spend their careers reporting the talk on Wall Street, elvish or human. (McCloskey \& Klamer, 1995, p. 194).

McCloskey \& Klamer stress the logic of talk instead of computation, because people make judgments and persuade each other whether a piece of information is knowledge (ibid., p. 191). The importance of talk contrasts with a main theme in investment theory, that of informational efficiency by which financial markets reflect all available information. But as discussed in Chapter 2, full immediate reflection of information is merely possible for obvious facts, because only then information can be processed mechanically without judgment.

\section{Klamer's View of the Culture of Economics}

The fact that economics ignores culture, does of course not mean that economics has no culture. In the current Chapter, I work along the lines of Klamer's (2001, $2003,2006,2007,2014)$ approach of the investigation of the culture of economics, 
which also suits with investment theory because it is a part of economics, aka financial economics. A difference between Klamer's and the current investigation is that Klamer investigates the culture of academics and I investigate the culture of the investment practice of institutional investors. So, what is culture? Culture constitutes the partly tacit social behaviour of a group, by Klamer described as:

[...] culture denotes beliefs, customs, values, emotions, stories, and sentiments that every member of group (Balinese men, Italians, academic economists) have in common and, crucially, by which they distinguish themselves as a group (Klamer, 2007, pp. 39-40).

Klamer's cultural approach fits in with the rhetorical approach applied in the dissertation, which has been Klamer's point of departure as well (1988 \& 1992 \& 1995 with McCloskey, 1994 with Leonard, 2001). Within the cultural approach Klamer concentrates on values and conversations. Values, or what is important, typify a culture and can be of a moral, social and cultural character - though originally, value is an economic notion (Klamer, 2003, p. 195). Value was a topic of debate in classical economics: is value about the exchange for labour as Marx thought, or about the subjective value for individuals like the marginalists proposed? In an effort to combine both theories, Marshall proposed that value arises out of the interplay between supply and demand (ibid., p. 195). In the language of economics values are called preferences, which are exogenous, given (ibid., p. 193). Yet, the reduction of values to mere preferences in economics, and the translation of preferences into utilities is not what happens when important decisions are made in real life, because values matter in decision making:

The consideration of values makes better sense of the process of choosing and acting, at least I will so propose (Klamer, 2003, p. 192).

Besides values, Klamer uses the metaphor of conversation to understand the culture of economics: 
This book [Speaking of Economics: How to Get in the Conversation] is the result of my finding out what it is that economists do, what makes the science of economics tick. The main point: think of economics in terms of a conversation, or, better yet, a bunch of conversations (Klamer, 2007, pp. xiii-xiv, his italics and my insertion).

Contrary to the academic ideal of truth, conversation as a metaphor reveals that truth is secondary (ibid., p. xiv). Conversations are highly disciplined, and for that matter "Conversations constrain what we are able to say (ibid., p. 26)." A feature of a conversation as a metaphor of culture is that it has a core which is stable for a lengthy period. Another feature of a conversation is that it discovers and develops. The conversation resembles Kuhn's (1962) paradigm, though the metaphor of conversation offers a language-oriented perspective, instead of a science-oriented perspective. The notion of conversation has been among others discussed by Rorty (1979) (Klamer, 2007, p. 18). Although a conversation resembles rhetoric, Klamer prefers conversation because it relates to a community:

[...] I prefer the metaphor of conversation. Seeking conversational commonalities is not a persuasive process but rather an attempt to find the expressions for my ideas to be heard. Conversation stresses the cooperative, the sharing of ideas, the identification with others (Klamer, 2007, p. 25).

Besides oral conversation, conversation consists mainly of individuals dealing with texts, such as articles and books. The past conversations are relevant to a better understanding of the current conversation and reveal options for a future conversation, as illustrated in Chapter 2 of the dissertation about the history of finance.

\section{Notions of Culture: Klamer and Hofstede Compared}

I will now compare Klamer's view on culture with that of Hofstede (1997), who has compared organisational cultures between countries. More in-depth, culture has according to Klamer three meanings, which I will quote at some length:

1) Culture in the anthropological sense [...] connotes the stories, history, expectations, artifacts, symbols, identities and values that a group of people 
shares and with which they distinguish themselves from other people. In this sense a family, company, city, region, ethnic group, nation, and continent can be said to have a distinct culture. Having a culture implies having the possibility to share the meanings of life with others. [...].

2) When the Germans write Kultur, they indicate the second meaning of culture: here culture connotes civilization usually expressed in the accumulated achievements of people in a certain region over a long period of time in the arts, the sciences, technology, politics, and social customs $[\ldots] .[\ldots .$.$] .$

3) In common parlor culture refers often to just the arts, sometimes including design, architecture and certain crafts [...]. [....].(Klamer, 2014, pp. 7-8, his italics).

Klamer's first meaning of culture is relevant for the analysis of economics and investing, and overlaps with Hofstede's notion of culture as a collective phenomenon:

Culture is always a collective phenomenon [...]. Culture consists of the unwritten rules of the social game. It is the collective programming of the mind that distinguishes the members of one group or category of people from others (Hofstede et al, 2010/1997, p. 6, their italics).

Hofstede describes national cultures by four categories: values, heroes, rituals, and symbols (Hofstede et al, 2010/1997, pp. 7-10). Values form the centre of a culture, and symbols its outer layer. Heroes are the role models of a culture. In investment practice, successful investors are role models, such as Warren Buffet and George Soros. In academic finance, heroes are for example Markowitz, Sharpe, Fama, and Merton. It is interesting to note that the investment heroes Soros $(1987,2008)$ and Buffet oppose modern portfolio theory. Rituals are social activities which a culture regards as essential, such as ceremonial meetings, and discourse, of which the dissertation provides an analysis. Heroes, rituals, and symbols form the visible practices of a culture. In Hofstede's categorization, Klamer's approach to the culture of economics focusses on values and the conversational part of rituals. 


\section{Culture and Uncertainty: Hofstede's View}

Hofstede has investigated cultural differences between countries in the research project for IBM. His explanatory model of culture has four dimensions (Hofstede et al, 2010/1997, p. 31):

1. Power distance

2. Collectivism versus individualism

3. Femininity versus masculinity

4. Uncertainty avoidance

The way a culture handles uncertainty is a part in Hofstede's explanatory model. He claims that countries differ in the way uncertainty is experienced and handled. Hofstede's dimensions and findings are debated by for example McSweeney (2002), see also Magala (2004), but for the dissertation the criticisms on his model are out of scope. But Hofstede's notion of uncertainty is relevant: if uncertainty relates to culture, culture could explain why and how investors cope with the uncertainty of investments. To Hofstede, uncertainty is a feeling of anxiety and a part of culture since the dawn of civilization. Civilizations have invented numerous ways to cope with uncertainty:

Every human society has developed ways to alleviate this anxiety. These ways belong to the domains of technology, law, and religion (Hofstede et al, 2010/1997, p. 189).

Technology has been directed against natural uncertainties, law against behavioural uncertainties, and religion against transcendental uncertainties. In investment theory, the technology of statistics treats uncertainty of the future outcomes of financial markets as if returns are a piece of nature 'out there'. 
As a matter of culture, uncertainty avoidance begs for predictive ability by some method:

Uncertainty avoidance can therefore be defined as the extent to which the members of a culture feel threatened by ambiguous or unknown situations. This feeling is, among other manifestations, expressed through nervous stress and in a need for predictability: a need for written and unwritten rules (Hofstede et al, 2010/1997, p. 191, their italics).

The psychology of uncertainty avoidance is that the method of handling uncertainty transforms uncertainty into a specified risk, which could imply two things: anxiety changes to a worry which is focused on the risk, or anxiety disappears (ibid., p. 197). Using a predictive method such as stochastical prediction handles uncertainty by replacing the feeling of uncertainty with a rational, technical method. Thus, risk per se is not the problem of uncertainty: the ambiguity of uncertainty is the reason of anxiety. Ambiguity aversion is also a subject of behavioural finance. Ambiguity aversion demonstrates why people prefer objective over subjective probabilities (Ellsberg, 1961).

\section{Culture and Uncertainty: Schön's View}

Donald Schön (1970) regards uncertainty and the quest for certainty, the stable state, as a central notion in human life. Culture creates stability and is a response to uncertainty. He perceives the aim for a stable state in both the secular and the personal domain. In the personal domain, humans tend to regard their identity as fixed when they say 'I am Dutch', 'I have studied economics', or 'I am a banker'. The belief in the stable state provides for a need:

Belief in the stable state is central, because it is a bulwark against the threat of uncertainty (Schön, 1970, p.1) 
The belief in internal or external stability constructs stability. According to Schön humans try to compensate uncertainty in one domain in stability in another, say, compensating the instability of being an artist with a structured way of life. When big changes occur in society three destructive reactions can occur: regression, revolution, and apathy:

There are a variety of responses to loss of the stable state. Some of the responses are anti-responses, versions of a refusal to recognise it, and they are destructive in character. One of them takes the form of a return. The idea is: let us return to the last stable state, to the way it used to be. [....]. There is a form of revolutionary response whose war-cry is total rejection of the past, but in such a fashion that the past is permitted to creep in by the back door [...]. There is a third kind of response, which is mindlessness (ibid., p. 6).

How about a constructive response to change? A constructive response is to learn how to adapt as a way of managing the change. My view on investing is that both the negative and constructive reactions to the economic and societal changes after the credit crisis of 2008, can be recognized in the behaviour of institutional investors: outright denial of the crisis, or trying to learn and improve behaviour. According to Schön, the solution to cope with uncertainty has to be both at the personal and the institutional level.

\section{2: Values, Decision Making and Phronesis}

I will now proceed by explaining how Klamer regards values and conversation as constitutive of decision making. Values and conversation are no part of the decision making in economics. The ethics of economics is reduced to the rational decision making with the help of calculations to optimize utility. The reduction of ethics to rational, utilitarian decision making is a result of scepticism about ethics. Finance follows the mechanical approach of economics, witness Markowitz (1952, 1959). Because the epistemology of investment theory fails, decision making in 
economics needs ethics. It is not to say that economics has no ethics, the point is that its ethics are constrained to prudence only.

In decision making, conversation is an alternative for calculation, calculation functions merely as the input for discussion. It is from deliberation between people that most decisions are being made. In the case that deliberation is too expensive, one sees that the decision is automated, for example for portfolio advice to private clients with an average wealth or less. Why is deliberation necessary? Could we not make a computer program in which we place all the inputs and calculate the outcome? The dissertation has stressed that the knowledge about the future is partial because of external uncertainty. Even if external certainty is a reasonably assumption, like in designing a bridge, the purpose, costs, design, aesthetics, etc. has to be deliberated as well. Yet, even more talk is needed if external uncertainty is at a higher level. We could say that internal uncertainty characterizes ethical deliberations, which needs to be explored by talking: "A reason to focus on the talking is the instability of the ethical categories (Klamer, 2009, p. 452)." The cause of instability is that values around which the decisions are made, must be interpreted in their situation.

Knowing one's culture means knowing one's values, which enhances decision making by deliberating values which are appropriate for the situation at hand. A value is not a means, but an end, a goal, in itself. For short, a value answers the question 'What is important?'. As a source of values, Klamer (2014) proposes the oikos, the home. He uses the oikos as a metaphor for the values that a person, an organization or the society strives for: oikos is more important than its translation into numerical entities in economics and in the market. In Klamer's words: "Doing the right thing is realizing our oikos, or whatever else we value (2014, p. 7)." Because values are part of a culture, culture is important in economics too. Klamer proposes to use phronesis or practical wisdom as a way of decision making that 
involves values. Phronesis here has a broader meaning than utilitarian prudence in economics, as phronesis is about the goals of life:

Practical wisdom, or knowledge of the proper ends of life, distinguished by Aristotle from theoretical knowledge and mere means-end reasoning, or craft, and itself a necessary and sufficient condition of virtue (Blackburn, 2008).

In Aristotle's ethics it [phronesis] is the complete excellence of the practical intellect, the counterpart of sophia in the theoretical sphere, comprising a true conception of the good life and the deliberative excellence necessary to realize that conception in practice via choice [...] (Taylor, C.C.W. in Honderich, 2005, their italics, my insertion).

Klamer uses phronesis in the Aristotelean meaning. The difference between the prudence of economics and the phronesis of Aristotle is that prudence of economics is reduced to self-interest, while Aristotle seeks the middle ground between self-interest and altruism.

Besides making decisions, phronesis is about the practical wisdom to realize values. Realizing values starts with being aware of the values. Klamer's approach reminds of Karl Weick's (2001) ideas about sense making within organizations under uncertainty. Weick illustrates that sense making is not like breaking the code in a game of Mastermind, but more like designing a map to make the world understandable from the perspective and the purpose of the maker (Weick, 2001, p. 9). Yet, decision making in neoclassical economics does resemble a game of mastermind, in which one solution is available by acting rationally. Sense making as cartography allows, however, many solutions because it is based on imagination within a social context.

How does Klamer's notion of values relate to McCloskey's approach of virtue ethics? Klamer says that consciousness of the seven virtues for discovering and realizing values is "most helpful for doing the right thing (Klamer, 2014, p. 26)." Klamer and McCloskey obviously agree in their criticism on the monistic approach 
of neoclassical economics, which reduces prudence to calculating utilities. According to Klamer, virtue ethics, as discussed in the Chapter 4, is a part of the deliberation about ethics and relates to values:

One is that virtue is a value that someone has internalized and enacts. The second is that virtuous action aims at the middle ground between two extremes, and the third is that judging virtuous behaviour requires situational knowledge (Klamer, 2009, p.453).

Where do McCloskey and Klamer differ? Klamer seems to pursue a Socratic approach to value, an approach to discover what is important. McCloskey's account of virtue ethics (2006) proposes seven virtues which are essential for a fulfilling life.

Klamer (2014) seemingly stresses the oikos, which may resembles the virtue of love the most, and phronesis, practical wisdom, while McCloskey seeks to balance the seven virtues. Further, Klamer applies phronesis to the practice of decision making within families and organisations.

Both McCloskey's virtue approach and Klamer's value approach can compensate the lack in epistemological strength of investment theory. McCloskey's approach tries to ensure a balanced approach by reminding us of all the seven virtues, Klamer's approach is a way of sense making for individuals at home or in an organisation, and is embedded in the virtues that McCloskey proposes. Both approaches stimulate conscious thinking about decision making. For the practical purpose of making investment decisions, formulating values within a virtue ethics context is important, though the starting point is insight and honesty about the epistemological weakness of mainstream investment theory. Merely reformulating the implicit dogma's of investment theory as values would be a mistake, and strengthen the received view of investment theory. 


\section{3: Methodology of the Investigation of the Culture of Investing}

The analysis of culture helps to explain the behaviour of investors. The central claim of the dissertation that predictability does not apply to investing, raises the questions why investors hold on to their practices of prediction, and what constitutes the practices of investors, seen through the perspective of culture. In the following the methodology of the investigation of the culture of investing will substantiated.

\section{Culture of Dutch Professional Investors}

The current Chapter aims to describe the culture of investing by investigating the culture of the Dutch professional investors. Though investing often becomes a part of the popular culture during a boom in the stock market, professional investors at Dutch institutions manage the largest part of the investments of private individuals in the Netherlands. At the end of 2014, Dutch pension funds manage 1.209 billion euro, that is $88 \%$ of the total invested amount of 1.379 billion euros of Dutch private individuals and organizations (De Nederlandsche Bank, 2014). Some 2.000 investment professionals work at Dutch financial institutions as measured by the combined number of members of the Dutch Association for Investment Professionals and the CFA Society Netherlands ('Vereniging voor Beleggingsanalisten' and 'CFA Society Netherlands'). Investment professionals are specialized, they can be for example an Asset Liability Management specialist, a fund manager or an analyst for segments within equities, bonds, credits or private equity, a strategist, a risk manager, or a performance analyst. In spite of the specializations of investment professionals, the Chapter aims to identify what the investors share in their culture, instead of investigating the differences between the specializations.

In the practice of investment management, prediction and stochastical prediction are variations on a theme. A stochastical prediction is a product of scientific 
refinement, which is simplified into two dimensions in Markowitz's portfolio theory and the CAPM, or into one dimension, such as a quantitative strategy that tries to benefit from an anomaly of the efficient market hypothesis. A one dimensional prediction can also be grounded on less complicated statistics such as long term data, a theory of valuation, technical analysis, expectations of future economic growth, investment themes, or the central bank policy.

\section{Ethnography includes Personal Observations}

The method of ethnography is an appropriate way to perform an investigation into the culture of a community:

Ethnography is the art and science of describing a group or culture. The description may be of a small tribal group in some exotic land or of a classroom in middle-class suburbia. The ethnographer writes about the routine, daily lives of people. The more predictable patterns of human thought and behavior are the focus of inquiry (Fetterman, 2015, p. 184).

Ethnography is a form of qualitative research and implies that the researcher is a part of the community to be observed, and that the researcher conducts interviews and studies documents (Bryman \& Bell, 2011/2003, p. 426). For example, Hammersley \& Atkinson (1983) take a similar view of ethnography. In the investigation I adopt Klamer's framework to focus on values and conversations. The ethnographic method is needed because the values of investors are hidden and have to interpreted, and the conversations of investors have to be witnessed to be typified.

The ethnographic method grounds on the insider's view, the personal experience, which makes sense since: "[...] climbing unknown mountains is safer with native guides [...] (Magala, 2004, p. 8).” My experience as an investment professional in the Netherlands will be a source of investigation. In my professional life I have for the last 20 years been working in investment management as an investment advisor, analyst, and researcher. I performed my research activities for a large part at the Dutch fund manager Robeco. For the last 10 years, I have been working at 
Dutch pension funds as an investment and risk manager. In this role, I have become very familiar with the way Dutch investment professionals behave and argue. Using my experience has at the same time the possible objection of a personal bias. The investigation uses, however, a variety of sources, that is interviews, literature, and the material of the dissertation, which are assumed to balance the analysis.

\section{Interviews}

Besides participative observation, interviews are a source of ethnographic investigation. I conducted a number of interviews with professional investors in the Netherlands which serve as background material for the investigation into culture. In ethnographic research finding 'key actors' is essential to gain insight into the culture of a group:

Some people are more articulate and culturally sensitive than others. These individuals make excellent key actors or informants. [....]. Key actors require careful selection. They are rarely perfect representatives of the group. However, they are usually members of the mainstream - otherwise, they would not have access to up-to-date cultural information. Key actors may be cultural brokers, straddling two cultures. This position may give them a special vantage point and objectivity about their culture (Fetterman, 2015, p. 187).

The interviewees chosen can be regarded as such key actors in the Dutch world of institutional investors. They are experts on innovative approaches in investing, and try to cope with the problems of stochastical prediction or the culture of investing in general. The choice for focussing the interviews on experts on innovative approaches has the advantage of gaining a contrasting expert view on investing and thereby on its culture. At the same time, it is also relevant for the dissertation to find how the investment practice handles uncertainty in others ways than prescribed by investment theory and other traditional investment practices.

Concerning the interviews, a qualitative, semi-structured approach has being taken. Its intention is to get 'rich' answers (Bryman \& Bell, 2011/2003, p. 467). Rich 
answer are useful because values and culture are hidden. To validate the interviews, the resulting text of the interview has been reviewed by the interviewee. In the following the interview questions are presented:

1. How would you describe your investment approach, and which elements play an important role in your approach?

2. What is the difference of your approach with the standard investment approach based on modern investment theory by Dutch institutional investors?

3. How did you come to your investment approach?

4. Which parts of the non-mainstream investment theory are neglected in investment practices?

5. How important is statistics for your approach, and what role do statistics play?

6. To what extent does your approach predicts returns or results of the investment process?

7. What are your experiences with your approach in the institutional practice?

8. How could the existing culture of investing be changed in your opinion?

9. To what values or virtues should investors pay more attention in their investment decisions in the future? 
A particular study of internal documents could also have been a part of the ethnographic investigation. I have not explicitly used the source of internal documents, because such documents are undisclosed. Though, as an insider I am very familiar with such internal documents and as such, the documents function as background knowledge.

\section{Literature on the Culture of Investing}

The investigation into the culture of investing is, however, not necessarily restricted to the ethnographic method. I will for example also refer to literature on investing to enrich the notion of the culture of investing. The literature of De Goede (2005), Preda (2006), Galbraith (1954), and Shiller (2000) is well suited for the culture of investing. The purpose of discussing their books is to typify the culture of investing, and discover the values and conversations of investors. The works of De Goede (2005) and Preda (2006) investigate the inception of the current investment theory and its contrast with the earlier notion of investing as gambling. De Goede en Preda were also treated in the Chapter 2 about the history of investment theory. Galbraith (1954) wrote a telling book about the stock market crash of 1929 and its aftermath. Shiller (2000) has investigated bubbles on financial markets and their (cultural) causes. The other Chapters of the dissertation are, of course, a relevant source as well. To my knowledge, there is no literature on the culture of Dutch professional investors at pension funds.

Because I focus on the culture of Dutch professional investors at pension funds, I will not discuss the literature that discusses the culture of investment banking, that is the culture that is associated with the culture of 'Wall Street'. Though both Dutch professional investors at pension funds and investment bankers deal with financial markets, the culture of Wall Street is in my opinion not representative for Dutch professional investors: a pension funds and a commercial American or global investment bank differ in the type of environment. The reader interested in 
the culture of Wall Street is referred to Michael Lewis $(1989,2010)$, Gillian Tett (2009), and Karin Ho (2009).

\section{Pitfalls of an Investigation into Culture}

In a paragraph about the methodology of the investigation of the culture of a group, the notion of culture as such needs to be discussed as well. In the following, the notion of culture will be discussed along the lines of McSweeney's criticism (2002) of Hofstede's (1997) cross-cultural approach. Though McSweeney's criticism is directed against Hofstede's methodology of cross-cultural research, it can be helpful for the current investigation because his criticism has a number of generalizable elements. McSweeney's (2002) criticisms, complemented with views of Magala (2005) and Morgan (2006), and including my response, are summarized below:

- Other factors than culture can explain actions (McSweeney, 2002, p. 109). Instead of culture, other explaining factors of the behaviour of investors could be the need to make a living, the commercial or bureaucratic ends of an organization, power relations, constitutional arrangements, or peer group pressure. My Response: I consider culture as a metaphor, a constitutive perspective of looking at phenomena (Morgan, 1986). Though Morgan focusses on the culture of organizations, instead of a professional group such as investors, his ideas are helpful in understanding culture as a means of investigation. Morgan (2006/1986, pp. 140-147) identifies as strong points of the metaphor of culture that it focusses on symbols, identifies shared values and beliefs, and promotes the notion of social construction. The danger of using the metaphor of culture might be that 'everything' is explained by culture. Yet, the point of using a metaphor is that it is appropriate, not that it could prevent other ways of seeing. The limitations of the metaphor of culture will in the dissertation be balanced by the outcomes of the other approaches applied, such as the rhetoric and 
the history of investment theory, the philosophy of statistics, the economics of uncertainty, and, the mainstream and alternative theories of investing.

- Assuming one uniform culture would disregard the diversity within the culture (McSweeney, 2002, pp. 95-96). My response: Dutch professional investors are a rather heterogeneous group, and the outcome of the investigation does not deny diversity within the culture. The cultural picture of investing will, of course, always remain a generalization. Yet, the point of the investigation is to deliver a persuasive picture of the dominant culture of investing.

- Culture is an elusive subject to grasp (McSweeney, 2002, p. 108). His advice is to be careful to take the outcomes of an investigation as being the culture. My Response: a concept such as a culture can be interpreted in many ways; the interpretation of the concept of culture does not differ from that of concepts such as freedom, rationality or truth. So, the culture does indeed not exist. Klamer provides a clear approach to the culture of economics by focusing on values and conversations, which he finds relevant to economics: he does not seem to claim that values and conversations describe the entire culture and differs in that respect from Hofstede's approach.

- Beliefs are not stable, but depend on the situation: so culture interacts with the changes in the environment, it is therefore dynamic (Magala, 2005, $p$. 9). My Response: in the literature review the inception of the current culture of investing and the culture of investors at the time of the crash of 1929 will be treated. One can wonder whether the culture of professional investors could become instable easily after all the financial and other crises that it has survived; as I see it, the culture of investing and its destiny root in the continuance of our liberal, free market society. 


\section{4: Personal Observations}

In line with the tradition of participatory research in ethnography, I will use my observations about investment management to construct a picture of the culture of investing. The picture has gradually formed as it took time to gain insight into the epistemological flaws of investment theory and practice. In 1991, I obtained an MSc in finance (in Dutch: drs.), and in 1999, I finished the postgraduate VBAeducation, the Dutch CFA-equivalent for becoming an investment and financial analyst. In my professional life I have for the last 20 years been working in investment management as an investment advisor, analyst, and researcher. My research activities I performed for a large part at the Dutch fund manager Robeco. For the last 10 years, I have been working at Dutch pension funds as an investment and risk manager.

My research interest in investment theory has been in the mathematical-statistical analysis of investment problems after I had written a Master thesis on alternative risk measures in Modern Portfolio Theory. Besides my interest in finance, I am also attracted to arts. In 2010, I completed a BA in Philosophy. My dissertation combines my interest in finance and philosophy. Besides having been a researcher with a mathematical-statistical bent and an interest in philosophy, I worked as an investment strategist and equity analyst, and have been advising clients on investing. Working in the practice of investment management and having analysed investment theory for practice, has taught me about investment theory, investment management, and the culture of investing.

My first experience in the practice of investing was as an investment advisor for private individuals at the ING Bank. At that time in the second half of the 90s, share prices rose sharply: I was advising in the middle of a bull market. To be an investment advisor, one had to be in command of the arguments for buying stocks in general (the positive returns in the past, the optimistic outlook) and for buying 
specific stocks (what to buy and why), understand the investment instruments such as bonds and options, and importantly, follow the news and interpret the stock market. Being an investment advisor was all about information, interpretation, talk and persuasion. Besides the ideas about investing from the bank, the diversity of clients such as entrepreneurs, professors, and public accountants, taught me different pictures of investing. The ideas about investing ranged from:

- investing is mere speculation, the market is autonomous: the irrationality argument

- the investment advisors knows better because they had time to study the market: the information argument,

- investing was about timing, about being smarter than others: the timing argument,

- investing was about a rational top-down allocation: the rational construction argument

- a patient long-term approach to investing leads to the best results: the long term argument.

In the 90s, after a long period of price increases, most investors did not believe that stock prices could fall deeply. An older investment advisor warned me that investment advising was a cyclical business: he had experienced the bear market of the 70s with the oil crisis and the high inflation. Despite knowing about the episode, I could not imagine a bear stock market in the future. If the stock prices fell, it was an opportunity to buy, to buy on the dips. Some investors were caught by the folly of the stock market. I had lengthy discussions whether buying call options with a long horizon was a better idea than buying the underlying stocks. Indeed, on the premise of rising stock prices, leverage pays off. Buying call options with a long horizon did, of course, not turn out to be a good idea in the longer run. As an investment advisor, I have gained experience with the practice of selecting stocks for a portfolio. My experience was that the strategies based on 
value, growth, themes, sectors or whatever often turn out differently than expected beforehand, for example other shares performed better (such as IT stocks in those days), the portfolio contained the wrong stocks within the sectors, or the nature of a stock or whole sector changed.

In 1997 I became an equity analyst. I was convinced that being an analyst I could better predict stock prices, because I would get a better understanding of the companies involved. After a number of analyses of stocks, however, it appeared to me that more knowledge about a company did not lead to superior ideas about the future performance of a stock. The translation from analysis to advice was often done by rules of thumb, about for example price-earnings ratio compared to expected growth rates, which could be made without the analysis as well. In order to work as an analyst, you have to believe in the idea that the work has value, or at least you must have fun in studying companies.

So in 1999, I continued my career as an investment analyst at the Dutch fund manager Robeco. As an investment analyst, I had the opportunity to explore the mathematical-statistical part of investment theory, and apply it to practice. I have thought about questions such as: 'How does time relate to risk in investing?', 'Are options useful in asset allocation?', 'How is risk perceived?', 'How to measure risk?', 'Does diversification pay off?', 'How to establish an expectation for future returns?' I experienced that the mathematical-statistical approach delivers insight about the mechanisms it studies, which has value as a way of interpretation, understanding. I have also learned that the investment industry upholds a number of myths that seem to be believed by most investment professionals. I mention a number of them below:

- A long investment horizon diminishes risk. Yet, investment risks are not smaller but larger with a long horizon, unlike what is often thought. As explained in Chapter 2, risks adds up on a longer horizon: the notion of 
more risk opposes the belief of investors that getting results from investing is merely of matter of time.

- The diversification of investments is the key to reducing risk. But the effects of diversification within and across asset classes is a myth: in times of crisis, it will not work because the correlations between the asset classes converge to 1 . Moreover, the rationale behind the low correlation coefficients is often not based on economic rationale.

- In the long term an investment portfolio will achieve its expected return. If the expected risk and return could be estimated, then it is unlikely that the expected return on equity is achieved in the long term. Expected returns are almost never met simply following the theory of statistics. Suppose we know that the expected return on equity is $10 \%$, then the probability is $50 \%$ (at each horizon) that the return is $10 \%$ or more. However, a yield of less than $10 \%$ also has a probability of $50 \%$.

In the beginning of the 2000 s, the bubble in the stock market started to burst. For the first time, I started to doubt that investing in stocks would always lead to a superior return above bonds. Since the bursting of the internet bubble and the three bad years of 2001-2003, I finally lost the belief that stocks would always yield more than bonds. The belief that stocks yield more than bonds, appeared to be an unconscious assumption of the investment world and its theory. I had realized that the equity premium above risk-free bonds was uncertain, and possibly not even positive. Obviously, personal experience changes beliefs.

At that time it seemed to me that investing in a broadly diversified investment fund was a better idea than investing in a particular portfolio. But I no longer believed in an active policy of the fund manager of the fund. No doubt, fund managers themselves believe that they can outperform the market, of course, if you do not 
believe that, you cannot be part of the culture of investing. I remember that a fund manager was under pressure, because one of the flagship funds under his management, underperformed for a number of years. The fund manager asked, in order to cope with the criticism in the weekly meeting of fund managers and research analysts: "Who does not believe in outperformance?" Of the some 100 people in the meeting room nobody raised his hand (me neither). The fund manager thus concluded: 'Fine, so that issue is settled.' If we all believed in outperformance, than the flagship fund would be all right, if merely given some time. It would have been possible to raise my hand in that meeting, but in the culture of investing at an active manager, that would have been unwise. You have to believe in outperformance to be part of the culture of investing at a fund manager.

I also have been an investment strategist at the fund manager, while continuing to do analytical work. Maybe, the perspective of looking at the financial markets as a whole including the economy, and trying to say meaningful things about it, could improve my insight in the financial markets. As an investment strategist, getting a clue about the direction of the economy was the key thing: it was all about the business cycle, recessions, and the central bank decisions about interest rates. But also was being an strategist broad and eclectic, for example about Minsky's model of boom and bust, societal trends, innovation, politics, and models composed of indicators about valuation, technical analysis, momentum and the like. Yet, despite that it was interesting to study economic phenomena, it did again not take long for me to conclude that the development of the economy is not predictable, just like the financial markets.

Since 2005, I work as an advisor to the board of a pension fund about investment and risk decisions. I have, for example, participated in more than 100 meetings of investment committees in which professional investors and trustee participated. In such meeting the investment policy is discussed, varying from Asset Liability 
Management studies which support the long term asset allocation decisions to short term tactical decision making, with discussion about what the economy, the stock market or the interest rate would going to do. My observation is that the culture of investing of pension funds in the Netherlands is not that different from the investment culture of a commercial fund manager. The biggest difference is that commercial reasons can influence the behaviour of investors at a fund manager: it can push people to be more optimistic about investing. Another observation is that the changing of the investment climate after the crisis of 2008 did not really change the culture of investing.

To summarize my ideas about the beliefs of the institutional investment community in the Netherlands:

1. Optimism that stocks will outperform bonds. The optimism continues in a bear market

2. More general: the economy and the financial market will return to an equilibrium

3. It is possible to outperform a market index, but it takes expert knowledge

4. It is possible to predict the market, but it takes expert knowledge

Due to personal character traits, some professionals in the investment world will leverage the beliefs. When asked individually, investment professionals will probably take interest in my views, but for reasons of culture, conformity, income, status and position they cannot express their doubts in public as it places them outside the community.

\section{5: Literature on the Culture of Investing}

The purpose of discussing literature is to typify the culture of investing, and discover the values and conversations of investors. The works of De Goede (2005) 
and Preda (2006) investigate the inception of the current investment theory. Galbraith (1954) wrote about the stock market crash of 1929 and its aftermath. Shiller (2000) has investigated bubbles on financial markets and their (cultural) causes.

The perspective that financial markets resemble gambling is old, witnessed by the De la Vega's (1688) eyewitness report of the Amsterdam Exchange in the $17^{\text {th }}$ century. Yet, nowadays investing is not perceived as gambling because it has a rationale, arguments to believe that it is worthwhile. Finance is now seen as a respectable scientific and societal subject, instead of as gambling, and at times associated with fraud and manipulation. The change of perception of finance from gambling to a rational activity took time and was forced by the emergence of the financial markets, of which the actors needed respectability (De Goede, 2005, p. 48).

An often heard argument in the conversation about investing is that idle money on a savings account should be replaced by investments, because the money should, instead of doing nothing, be activated in the financial market. In the financial market, money can be put to work. De Goede underpins that the distinction of finance and gambling is merely political, ideological, instead of based on the nature of financial markets:

Like statistics, modern (financial) rationality is not wholly secular and disinterested, but is premised upon faith in a divine or cosmological order (De Goede, 2005, p. 114).

She identifies a transcendental justification of investing, which is based on faith, on optimism that capital invested in the stock market always will accumulate. Interestingly enough, she also regards statistics as a transcendental faith. 
Preda has described the process of the transformation of the notion of investing in detail. In France in the 1860s investing in stocks is associated with gambling, but how could investing in stocks be legitimized (Preda, 2006, p. 151)? The legitimization of investing in stocks is done by vernacular science. The vernacular science of financial investments attempts to dispose the unethical connotation of investing by turning it into a rational and partly scientific object, in which information becomes the most important aspect (Preda, 2006, p. 150).

Vernacular science uses among other things the analogy of financial markets with physics and engineering, and adapts the idea of rational behaviour as the guideline for stock holders. Rational behaviour in the form of self-control and the study of information, is promoted as the key to successful investing. A part of vernacular science results in the efficient market theory because it includes probabilistic and abstract reasoning. The perspective that financial markets can be managed by a scientific approach is enforced since 'modern' finance emerged in the 1960s.

\section{Galbraith's The Great Crash 1929: The Classic Study of that Disaster offers} insight into the culture investing in the $20^{\text {th }}$ century. The magnitude of the stock market crash of 1929 compares with the consequences on the financial markets following the credit crisis of 2008. In the opinion of Galbraith (1992/1954, p. 9), regulation nor ethics will end the boom and bust cycle, he advocates to keep the history of the cycle of boom and bust alive by retelling the story how investors came to believe in the illusion that the stock market is an utopia in which stock prices continue to rise:

For protecting people from the cupidity of others and their own, history is highly utilitarian. It sustains memory and memory serves the same purpose as the S.E.C. and, on the record, is far more effective (Galbraith, 1992/1954, p. 11).

The metaphor that financial markets are bubbles, highlights the danger of the financial markets. Socially constructed optimism regularly pervades the culture of 
investing. Some phenomenon causes, though not justifies, the optimism: "[ $\ldots]$ speculation does not depend entirely on the capacity for self-delusion (ibid., p. 33)." In a boom time, the culture of investing is a part of the popular culture. A condition for a boom is that investors have trust:

Such a feeling of trust is essential for a boom. When people are cautious, questioning, misanthropic, suspicious, or mean, they are immune to speculative enthusiasms (Galbraith, 1992/1954, p. 188).

Besides trust, a boom needs prosperity: ample resources have to be available to be invested (ibid., p. 188).

The optimism of investors in a boom, makes being negative on the outlook of the stock market a taboo. Galbraith experienced the taboo personally: he received various threats of physical violence after he testified in 1955 at a congressional hearing that the stock market crash of 1929 could repeat itself, at which the stock market reacted negatively (ibid., p. 17). The threats received are an obvious reaction to breaking a taboo because the declining stock market threatened investors's optimism. The culture of investing combines personal interest and pressure of the group, and rejects critics:

Clearly, given the nature of the euphoric mood and the vested interest therein, the critic must wait until after the crash for any approval, not to say applause.

To summarize: The euphoric episode is protected and sustained by the will of those who are involved, in order to justify the circumstances that are making them rich. And it is equally protected by the will to ignore, exorcise or condemn those who express doubts (Galbraith, A Short History of Financial Euphoria, in The Essential Galbraith, 2001, p. 254).

People just want to believe they can get rich. And some investors do get rich, if they manage to sell their stocks before the market collapses.

A part of the culture of investing is that its optimism cannot afford doubts.

Therefore no forecaster is easily held accountable for his optimism. An exception 
was, as told in Chapter 2, Irving Fisher, who is even nowadays remembered for his unfortunate prediction in October 1929 before the crash that "stock prices have reached what looks like a permanent high plateau (Dimand, 2007, p. 45)." As a matter of culture, the sayings of experts are part of the ritual of investing.

Shiller (2000) is an opponent of the efficient market theory and elaborates on culture in Irrational Exuberance. The public generally expects stocks to deliver a positive return in the long run, an expectation based on the views of experts and other people:

It is still very much the conventional wisdom today that for investor whose horizons are years long, the stock market cannot disappoint. People modify their sense of this conventional wisdom only slowly as they hear expert opinion from time to time, hear others say 'they say that ...,' and hear others express approval or disapproval of these opinions (Shiller, 2001/2000, p. xiv).

The optimism of investment experts that the market goes up in the long run, is based on background facts, which are not disputed (ibid., p. xv). The interesting thing is that after a crash the optimism remains (ibid, p. xvi). The remaining optimism is typically a cultural phenomenon of investing, a value that guides investors as they perform their cultural program.

The media offers support for the optimism about the stock market, which is by closer inspection performed by a typical conversation in which the interviewer asks critical questions and is reassured by the interviewee (ibid, p. xv):

Interviewers and investment professionals sometimes seem to play a sort of rhetorical game on television that plays out pretty predictable to be supportive of the market. The interviewer asks dark questions about whether the market might conceivably do very badly, blunt questions posed as if to get an answer with the plain, unvarnished truth. The interviewee answers in an assuring, confident, professional manner about the great longer-run outlook for the market, about the importance of not being unsettled by short-run fluctuations, and about the benefits of holding firm to a long-run investment strategy. The interviewer establishes his or her news-media credibility as pressing hard for the truth, but given the typical choice of interviewee, the interview closes on a suitable upbeat note (Shiller, 2001/2000, p. Xv). 
The point of the long citation is that, in my view, it could be a conversation between a client and an investment expert or advisor as well, and could be typical for the culture of investors. The client could be less critical than the interviewer but nevertheless worried, and is being reassured.

The media play a decisive role in the culture of investing. Though they look like objective observers, they spread the ideas about the markets. Media compete about the attention of the public and want to keep that attention (ibid, p. 72). How can they do that? The news has to be interesting and preferably be a continuing story. The stock market is a source of continuing information, just like sport. Thus, the media need the stock market to capture the attention of the public, which cannot get enough of news about the stock market during the boom days and when financial disaster strikes. Markets are, however, not moved by the news as you would expect markets to be. Shiller explores the crash of 1987 as a case study and finds that no significant news could be discovered as the cause of the crash (Shiller, 2001/2000, p. 88). News is brought because the public wants explanations. The media also help promote the optimism of the stock market, because that is the story that the public wants to hear.

Another point of culture is that in the good times of the stock market a belief exists that a new era has come, which elevates growth forecasts to a higher level, and underpins rising stock market levels and valuations such as price to earnings (ibid., p xxii).

\section{6: Innovative Case 1 The Management of Investment Risk}

The culture of investing is heterogeneous, also because innovation takes place in investment practice. The innovations of interest here is how practice constructively handles the uncertainty of investing. The current paragraphs will treat the ideas and practices of three investment professionals in the institutional part of the Dutch financial sector dealing with mainly pension funds. Each of the interviewees has 
an innovative way of dealing with the problems of the epistemology of investment theory, i.e. the stochastical unpredictability of future returns and the culture which holds on to the practices of investing. The interviews serve as background material for the investigation into culture.

\section{Introduction Theo Kocken}

Theo Kocken is Professor of Risk Management for Institutional Investors at the VU University Amsterdam. After working as a risk manager at ING and Rabobank, he became in 2000 a founder and CEO of Cardano, a Dutch company which performs risk management services for the financial sector such as pension funds. In 2007 an asset and risk management branch of Cardano in the United Kingdom was launched. Currently Cardano employs some 150 people (Cardano, 2015). Cardano's risk management approach aims to avoid excessive risk taking. What 'excessive' means, depends, of course, on the theory of risk that Cardano applies. Besides servicing risk management for pension funds, the firm advises for example about the design of pension fund schemes and the distribution of risk between generations. The text following is based on my interview with Kocken, conducted on 9 February 2015, and is completed with additional information from the literature and other sources.

\section{Risk Management}

When Kocken was 30 years old and head of market risk at ING, a large Dutch financial institution, he had experienced that financial markets in South America suddenly could collapse and that past statistics cannot predict such cases. The experience made him reflect on the usefulness of Markowitz's portfolio theory and of statistics for the risk management of financial markets. A story that he has frequently told at seminars about risk management illustrates why Value at Riskmodels (VaR) are still being used despite their flaws. A VaR-model is based on portfolio theory and measures the maximum absolute or relative risk within two or three standard deviations of the current value of an investment. Financial 
institutions worldwide practice the method of VaR. As risk manager he calculated the VaR of a certain portfolio on a $99 \%$ confidence interval for a 10-day horizon, which was mandatory for the De Nederlandsche Bank (the Dutch central bank, for short DNB). For ING, however, he conducted a stress test for the same portfolio with a significantly higher outcome than the VaR for DNB. Yet, DNB did not use the higher level of possible loss as proposed by Kocken, not because they did not understand or disagreed to his interpretation of the risks, but because they had to conform to the supervisory rules. The conversations with DNB about how to estimate risk were an eye-opener for him; intelligent professionals seem to have to neglect common sense because usual practices dominate.

Kocken (1997) has formulated an alternative, innovative approach of risk management in his book about risk management for financial institutions. The book treats, among other things, subjects such as value at risk and the risks of options. Yet, one chapter of the book, that is chapter 7 about stress testing and the measurement of event risk, differs from the usual view about risk management centred on the VaR-approach. The reason for including stress tests in his book is that VaR underestimates risk. Kocken calls the phenomenon 'the Value at Risk paradox': the VaR-model does not function when it is needed most, that is in times of crisis when risks materializes (Kocken, 2010/1997). The stock market crash of 1987 demonstrated risks in the form of standard deviations of 10 times and more, which the VaR-model merely did expect to happen once in a period from the big bang until now (ibid., p. 213). One of the reasons that VaR-models fail in times of a financial crisis is the assumption that returns are normally distributed. Though VaR-modelers have tried to incorporate non-normality, in practice the VaR-models remain too optimistic.

So, why do his stress test demonstrate a higher possible loss then the VaR-model? The higher loss originates from rare events, which are no part of past data on which the VaR-models are based. For example, Brady Bonds (restructured bank loans of 
mainly South American countries) can behave normally for twenty years, but then suddenly collapse by $80 \%$. The collapse of the Argentinian peso is another example in which past statistics are meaningless, because if the Argentinian peso is decoupled from the American dollar, past statistics based on a coupling of the two currencies are no longer representative. Kocken thought a decrease of 50 to $60 \%$ possible in the cases mentioned. Interestingly, professionals in the financial world have often reacted that stress testing is not scientific, because it is based on economic judgment, and not on statistics. The received view of risk management practitioners expresses the ideas of modernistic science, which have been discussed at length in the current dissertation. Kocken's approach of stress testing (2010/2007, p. 223) involves a casuistic approach based among other things on macroeconomic and political research, and demands experienced and creative risk managers.

Besides that the tail risks in financial markets are bigger than expected by the users of conventional investment theory, diversification does not function either in a crisis on financial markets. Diversification does not function because correlations tend to move towards their maximum of 1 in a crisis, which means that no diversification benefit is left. The accepted theory, however, is Markowitz's theory of diversification, which has proven to be naïve if merely backed by past statistics instead of an economic rationale. An example of diversification with an economic rationale is $T C X$ (the currency exchange fund), which is supported by Cardano Development (a branch of Cardano). The fund provides loans in local currency to entrepreneurs in countries in West Africa, East Africa, Central Asia, and South America. The fund hedges the local currency risks by diversifying 50 currencies, which are statistically independent because the dynamics of the countries involved differ. Such an economic rationale for diversification does not exist for stocks and bonds of the US and Europe, because in a crisis the markets of the US and Europa move in the same direction. 


\title{
A Economic Explanation of the Failing of Portfolio Theory
}

Concerning economics, Kocken (2012) advocates the theory of Hyman Minsky which is also of importance for risk management. Minsky's theory (1982) sketches the causes of economic instability that lead to a worldwide depression. Minsky stresses that the economy and financial markets do not tend to an equilibrium as neo-classical economics and finance propose, but that the economy and financial markets are inherently instable:

\begin{abstract}
Minsky realised that our economy was anything but a system where external shocks from outside our assumedly stable economy are processed by the economy until equilibrium is re-established. In fact, the world works exactly the other way round. Big unexpected external shocks may be troublesome, but they are not the reason behind real deep depressions. The major destabilising moves are created internally, from within the economy. A process that keeps repeating itself, hence the name endogenous instability. It is those endogenously created moves that lead to depressions (Kocken, 2012, p. 7).
\end{abstract}

The instability is created by superfluous lending which fuels optimism, and will eventually lead to an endogenous crisis. See also Chapter 2 of the dissertation in which the theory of bubbles is explained. The policy applied by the central banks to act on recessions by lowering the interest rate, postpones and thereby aggravates the crisis.

Kocken relates the optimism of investors to the behavioural bias of the affect heuristic:

This is partially due to overconfidence but is also supported by the 'affect heuristic', as researched by Paul Slovic: when people have a good feeling about the returns of the markets, their perception of the risks in the markets is lower.

This is exactly what we saw during many crises in the past: the more risk accumulated (exploding stock and house prices etc.), the lower the volatility in the markets (Rosenberg \& Kocken, 2013, p. 31). 


\section{A Behavioural Explanation of the Failing of Portfolio Theory}

Kocken has found behavioural finance illuminating as a source of explanation why professionals continue to use models based on portfolio theory; endogenous instability can be explained by behavioural finance as well. In his inaugural lecture (2012) as a Professor of Risk Management for Institutional Investors at the VU University Amsterdam, he combined Minsky's theory of economic instability (1982) with the insights of behavioral finance, which are directed at individual behaviour.

A number of behavioural biases are central to explaining why investment and risk professionals carry on with the models of portfolio theory. The first one is ambiguity aversion as described by Ellsberg (1961): people dislike uncertainty and prefer a presentation which is more elaborate (also see Chapter 3 of the dissertation, in which Ellsberg's views are discussed). Risk professionals arguably understand the criticism, but taking the criticism seriously would force risk professionals into ambiguity. That is why risk professionals find it difficult to abandon models because models are their main support against uncertainty.

The second behavioural bias is the so-called attribute substitution in which a problem is simplified by reducing the problem to a simpler problem, for example in representing the economy by a neo-classical equilibrium model or financial markets risk by a VaR-model:

[...] our brain has the latent tendency to substitute complex problems with less complex problems. And these less complex, though often mathematically rigorous problems are then resolved as a substitute solution for the complex problems. That is called attribute substitution (Kocken, 2012, p. 18).

The third bias relevant to risk management is overconfidence, which paradoxically has the tendency to rise if the level of uncertainty is higher. Overconfidence could also explain the inability to learn from financial crisis (Kocken, 2014, p. 19).

Despite the economic depression in the 1930s, economics has embraced the Cartesian mathematical approach in the 1950 s which does not include the 
phenomena of depression and its causes. Yet, the point is that mathematics does not support predicting in economics and finance. Models for the calculation of option prices do not predict: a low implied volatility as distilled by an option valuation model does not mean that the volatility of the underlying asset will remain low. Though, mathematics is useful for the valuation of options on financial markets or embedded options in pension schemes, because uncertainty can be articulated by the mathematics of option theory. A further treatment here of embedded options would be out of scope, an example of an investigation of embedded options and its redistributive effects on pension funds can be found in Kocken (2006).

The fourth relevant bias is the confirmation bias: the confirmation bias is the tendency to filter information towards a confirmation of our point of view. It helps to explain why people do not always seem to learn from new information: they ignore the new information because it does not match earlier adopted ideas.

\section{How to Invest}

The assumptions of the models of Markowitz and Sharpe that probability distributions and correlations are known and therefore predict, are flawed. The consequence is that investors following portfolio theory will become disappointed, because portfolio theory embeds optimistic assumptions. Thus, investing in a more robust way has to be done in another way:

And if we concede we don't know the distributions exactly, then we should steer in a very different way to lead to more robust solutions (Rosenberg \& Kocken, 2013, p. 29).

Assuming a pension fund has a clear ambition for the realization of the pensions, for example about the aspiration level of the indexation, then, making investment portfolios robust, starts with investigating what negative consequences are unacceptable. In practice, the question for pension funds what level of the funding ratio, or level of indexation, is unacceptable, appears difficult to answer. But, if a 
pension fund does not answer the question what level of funding ratio is unacceptable, chances are that the fund has taken insufficient risk measures to prevent happening that the funding ratio ends up below such a disaster level. Once a disaster level has been formulated, the arrangement of the investment portfolio has to prevent that the disaster level will be met. To the purpose of risk management, 5 stress scenarios are projected on the portfolios. The projection is both top-down and bottom-up. The bottom-up projection is relevant because the actual implemented portfolios of the investment managers may react differently to the stress scenarios than the top-down portfolio strategy had assumed. The purpose of the stress scenarios is to assure that the pension fund can survive the worst of the five scenarios.

\section{The Use of Options}

What is the role of derivatives in the strategy of Cardano? Derivates can be applied for managing the interest and inflation risk of pension funds, but also for investments in stocks. Pension funds in the Netherlands have usually a large amount of stocks in their portfolio. Because of peer group pressure in the form of reputation risk, Dutch pension funds may find it hard to significantly lower the amount invested in stocks. The investment approach favoured by Cardano, is performed by the Cardano UK branch: Cardano UK composes a portfolio with many small bets, with a small amount of stocks, and aims at a modest investment performance. If an investment approach with small bets is not possible, buying put options offers another possibility to reduce risk. Such downside protection with put options to protect a minimum value of stocks has a cost, but offers real risk reduction which fundamentally differs from the assumed risk reduction in portfolio theory.

The cost of buying put options can reduced by combining put options with writing call options on the stocks involved. A written call option means that the writer, that is the pension fund, receives a premium in exchange for the future delivery of the stocks or its financial equivalent at a certain price level. By writing call options 
the return of the stocks is maximized. Combining put options with written call options, aka a collar, provides risk reduction if stocks have to be held in the investment portfolio (Van Capelleveen, Kat \& Kocken, 2004).

An example of a case in which Cardano was involved was the protection of the funding ratio of the Pension Fund of the Rabobank (Walschots \& Capelleveen, 2008). The Rabobank Pension Fund acquired a hybrid protection, combining the coverage of interest rate risk (which stems from the long term obligations of the pension fund to pay pensions to the participants) and equity risk. In technical terms, the pension fund engaged in an equity linked swaption collars, which combine a collar on both the interest rate and stocks. It would be out of scope to explain the technical details of the approach, for that see Walschots and Van Capelleveen (2008). The construction was applied in 2007, and proved to be valuable in 2008 at the time of the credit crisis. The case of the Rabobank Pension Fund is rather unique, it takes courage and knowledge to execute such a protection strategy.

\section{What Should Be Done about the Current State of Finance?}

Having explained the problems with finance, a key question is how the theory and practice of finance can be changed. Kocken notes that Taleb, a well-known critic of prediction in investing, is widely read and admired in the investment world, but that his ideas are not put to practice. Why not? Apparently, investment professionals are not cynical or critical enough, and are not able to think out-of-thebox.

An important step in changing finance is that investment and risk professionals must realize that their field and its praxis is flawed. But current training ensures a continuation of the faith in current finance. Therefore, Kocken has launched a MSc in Risk Management for Financial Institutions at the VU University Amsterdam. The MSc differs from the usual quantitative approach of risk management and is intended for experienced professionals who have started to 
question the quantitative approach. The MSc in Risk Management has a multidisciplinary approach in which, besides assessing the regular risk models, consists of subjects such as psychology, philosophy, history of financial markets, decision making, and group dynamics.

Besides his educational effort, Kocken engages in reaching a wider audience for the failing of economics and finance. Therefore, he made the documentary Boom Bust Boom with Terry Jones, who was part of Monty Python, about human behaviour which is absent in the neoclassical economic models. In an article about the documentary in Ad Valvas, he explains to the students of the Amsterdam University VU that "economists live in a fantasy world (Schilp, 2015, p. 1, my translation)." Including human behaviour in economics as Kocken proposes, means to acknowledge that people imitate each other when they make decisions, which creates bubbles and instability. The documentary is aimed at economists, policy makers and the public. Kocken is now supporting student organizations that demand a more pluralist education (Rethinking Economics, Post-Crash Economics Society, Young Scholars Initiative of the Institute for New Economic Thinking). Their joint aim is to build a website for the public (its launch is targeted in the first quarter of 2016), explaining the economic news in laymen terms, but also offering different views on the underlying causes instead of one single view of 'this is how it works'.

Entrepreneurship in the financial services could change practice as well. The risk management services of Cardano have been a success: Cardano provides services to pension funds of which the assets amount to 150 billion euro (Cardano, 2015), but in terms of market share, Cardano is not dominant.

Another solution concerns the development of ideas employed in economics and finance. Kocken suggests that economics and finance should engage more in investigating the complexity of the economy and financial markets with a bottom- 
up approach. A bottom-up approach replaces the equilibrium analogy of physics by the evolution of biology, like adopted by the Santa Fe Institute.

\section{7: Innovative Case 2 Shell's Scenarios-approach}

\section{Introduction Peter Heijmans}

Peter Heijmans is Head of Strategy at Shell Asset Management Company

(SAMCo). SAMCo has some 60 billion euros of assets under management for defined benefit pension funds of Shell worldwide such as for the Netherlands, Great Britain, the United States, Austria, and Germany. Shell, or more formally Royal Dutch Shell plc, is worldwide active in energy and petrochemicals, and employs 90.000 people in 70 countries. Heijmans is trained as a macroeconomist and worked for 4.5 years at the Shell Strategy \& Planning department, in the Scenarios team, and prior to that as an economist in the US and for the Dutch government. The Strategy \& Scenarios team makes scenarios about the business environment of Shell and is concerned with subjects such as the oil prices, the energy demand, the global energy system, and the global economy.

Since August 2013 Heijmans is head of investment strategy at SAMCo. As the head of strategy, Heijmans can use his experience with scenarios for the investment management of the pension funds of Shell. The text following is based on my interview with Heijmans, completed with additional information from articles and other sources. I conducted the interview with Peter Heijmans on 20 February 2015 .

\section{Scenarios instead of Statistics}

Shell is a pioneer in scenario-thinking and has been working with scenarios since the 1970s. The attraction of scenarios is that they stress discontinuity, instead of the usual mechanistic claim of continuity provided by forecasting (Wilkinson \& Kupers, 2013). The company works with scenarios because scenarios instead of statistics enable to cope with uncertainty, indeed: 
Any form of mechanical extrapolation/modelling/probability analysis is 'an enemy to thinking'.

Probabilities assume the presumption of predictive accuracy. Scenarios are most powerful when they stimulate flexible and innovative thinking (Heijmans, 2014, p. 10, his emphasis).

For Shell the scenario-approach prohibits the usual behaviour of people when confronted with uncertainty: people usually dislike uncertainty and therefore ignore uncertainty (Shell, 2008, p. 9). Scenarios consider the worrying parts of uncertainty, are story-like, and prepare new conversations:

Decision makers can use scenarios to think about the uncertain aspects of the future that most worry them —or to discover the aspects about which they should be concerned - and to explore the ways in which these might unfold. [....].

Scenarios are based on intuition, but crafted as analytical structures. They are written as stories that make potential futures seem vivid and compelling. [....].

Scenarios are intended to form a basis for strategic conversation - they are a method for considering potential implications of and possible responses to different events (Shell, 2008, p. 8).

Shell has been working with scenario's because the route from discovery to exploration of and distribution of energy cover long horizons, and investment in projects are capital intensive and irreversible (Heijmans, 2014, p. 5). Depending on the subject the horizon of a scenario can stretch to 20 years for global themes such as mobility and cities, and more than 50 years for issues such as energy systems (ibid., p. 13). With such characteristics, the traditional way of forecasting nudges people into a direction that seems appropriate because of our education, training, and current dominant ideas (ibid., p. 7). Yet, experience shows that the future probably differs from such extrapolations, moreover such a focus tends to hinder other ways of seeing. A detailed description of the current and past scenarios of Shell (see www.shell.com/scenarios) would be out of scope for the dissertation. 


\section{Investing in General}

How does Heijmans look at the role of investing for pension funds? Investing is not a purpose in itself, but should be aimed at achieving the ability of SAMCo's clients to fulfill their nominal liabilities and the ambition to grant indexation. SAMCo's clients have a long term investment horizon. Return is a reward to risk, which needs to be taken, but in a careful and sustainable way. Diversification improves the trade-off between risk and return. Active asset management can add value.

Most pension funds of Shell have determined journey-plans for de-risking, mostly defined in terms of the funding ratio. De-risking in this context is defined as reducing the investment and balance sheet risk of the pension funds, with the objective of reducing the likelihood of a drop in the funding ratio in the future, and hence reducing the likelihood of the need for increased sponsor contributions. The goal is to reach a certain funding ratio threshold, at which the surplus of the fund is large enough to pay pension benefits including indexation for inflation. Once in 'happy land' the investments can be de-risked, because risk taking is not necessary anymore to reach the goal.

\section{Scenario Thinking Applied to Investing}

Working with scenarios differs from working with models because scenarios are meant to explore and models are meant to analyze. Models for investing are based on historical data. But in the future, discontinuities will appear and that makes the stochastic future different than assumed by the model. The credit crisis of 2008 illustrates the effect of discontinuity for the Dutch pension fund of Shell: the Asset Liability Management study (ALM-study) for the pension fund, conducted just before the crisis in 2008, resulted in a risk of less than $0,1 \%$ that the funding ratio could halve. Yet it did happen: the funding ratio of the pension fund declined from $180 \%$ to $80 \%$ (Stichting Shell Pensioenfonds, 2015, p. 69). So experience demonstrates that it is dangerous to found the investment policy on ALM. 
Though, an ALM-study has other qualities as it allows to identify the interests and risks for the various stakeholders, such as the sponsor and the participants, of the pension fund. For example, if the pension fund lowers the weight of stocks in the portfolio, then the probability that the sponsor has to pay additional premium declines, but at the same time a higher level of premium is necessary because the expected return of the portfolio diminishes because of the lower weight of stocks. And as a result, the average indexation, that is the compensation for inflation, for the participants will probably decline, but also become less risky. Thus, ALM allows to compare various investment and other pension fund policies in a stochastical way. One should however be careful to take ALM-results as absolute results: the results compare relatively. Heijmans does not take the outcome of the ALM-study literally that the average funding ratios eventually always will rise, because the rise stems from the fixed equity risk premium which is normally as an input for ALM. So, judgment of senior investment professionals is needed to interpret the results, and to consider other scenarios as well.

The scenario approach of Shell has had a first try-out for the investment management of the pension funds of Shell. The subject of the workshop for investment management with the Shell Scenario team was the outlook for inflation, which at the same time determines the interest rate, often the biggest risk for the funding ratio of a pension fund, because the pension obligations are sensitive to changes in the interest rate.

\section{How to Make Scenarios}

Shell has a lot of experience with making scenarios. How does making a scenario work in practice? A multidisciplinary expertise is essential to overcome the problem of having an incomplete view of reality (Heijmans, 2014, p. 9). For the workshop a certain type of people are needed. For example, if only people who are accustomed to ALM studies participate then the workshop does not result in interesting scenarios because the traditional answers will probably result. To make a workshop a success, a diversity of backgrounds is needed, because different kinds 
of thinking are needed, for example the thinking of political scientists or philosophers when the outlook for inflation is discussed. So external people from outside of SAMCo are being sought, preferably from other countries. To work with scenarios, senior people are needed and the participants have to be prepared, because people often feel comfortable with their acquired views.

Some 10 to 15 people take part in a workshop. A workshop in which the scenarios are built is based on conversation (ibid., p. 9). The workshop may appear a somewhat chaotic process. Various experts will present their view to an audience of Shell employees. Then discussion follows and the facilitator of the workshop collects and categorizes statements. During the workshop relationships between the statements are laid. Heijmans explains that a workshop on the same topic other could yield other, not necessarily better or worse results as well, the results possess an element of serendipity. Though what results from the process is out-of-the-box, non-traditional, and from different perspectives than the traditional model-based approach.

The scenario team prepares the workshop by identifying the important trends, for example for inflation demographic trends are an input. In the identification of trends, the so-called critical uncertainties, the most important uncertainties about say regulations, politics, or oil prices have to be discovered. In the workshop the experts are asked to present their view on how the critical uncertainties could develop. One caveat would be to merely present the extreme scenarios. But to stress extreme scenarios does not work because it leads to confusion instead of results from the workshop.

To illustrate the outcomes of the recent scenario exploration for the businesses of Shell, the scenarios 'Mountains', in which government policies dominate, and 'Oceans', in which the markets dominate, are presented here:

\section{Mountains}


The first scenario, labelled "mountains", sees a strong role for government and the introduction of firm and far-reaching policy measures. These help to develop more compact cities and transform the global transport network. New policies unlock plentiful natural gas resources - making it the largest global energy source by the 2030s - and accelerate carbon capture and storage technology, supporting a cleaner energy system.

\section{Oceans}

The second scenario, which we call "oceans", describes a more prosperous and volatile world. Energy demand surges, due to strong economic growth. Power is more widely distributed and governments take longer to agree major decisions. Market forces rather than policies shape the energy system: oil and coal remain part of the energy mix but renewable energy also grows. By the 2070s solar becomes the world's largest energy source (Shell, 2014).

\section{8: Innovative Case 3 Investment Beliefs}

\section{Introduction Alfred Slager}

Alfred Slager is Professor of Pension Fund Management at the TIAS School for Business and Society in the Netherlands. He has been working in investment management as a portfolio manager at Fortis Investments, policy advisor at PGGM, the Dutch Pension Fund for the Care and Welfare sector, and as the Chief Investment Officer of the Pension Fund of Stork. Currently he is a trustee of the board for SPH, the pension fund of general practitioners in the Netherlands. The text following is based on my interview with Slager, and is completed with additional information from the literature. I conducted the interview with Slager on 17 March 2015.

\section{What are Investment Beliefs?}

Slager has pioneered, together with Kees Koedijk $(2007,2011)$ the field of investment beliefs. The board of a pension fund or an investment manager has implicitly or explicitly beliefs, in other words ideas or convictions about investing. Investment beliefs consist of three parts: beliefs about the capital markets, the organization of the investments, and the society (Slager \& Koedijk, 2007, p. 77).

The reason that investors should examine their investment beliefs is because 
finance lacks the epistemologically 'certainty' of physics, and, though data on financial markets are available in great quantities, models of financial markets mostly lack predictive power (ibid., p. 78). The lack of an effective epistemology makes investment beliefs dominant for the investment policy executed in practice. Investment beliefs are, however, often tacit, and need to be clear to improve the performance of investing:

Investment beliefs improve stakeholder governance by reducing possible conflicts of interest, and charge the innovative adaptability of an organization by setting guidelines for best practice. Managers need to formulate their own investment beliefs: a clear view on how they perceive the way capital markets work, and how their organizations can add value and strive for excellence (Slager \& Koedijk, 2007, p. 77).

Another reason for the importance of articulating investment beliefs, is that changing beliefs is easier if the beliefs are known. This knowledge is instrumental for effective reflection on the results, and the (potential) need to adapt in changing financial markets. Beliefs have to be measured if possible: ex-ante as evidence to support a belief, and ex-post to sustain to a belief or to change it.

The approach to formulate investment beliefs originated from the practice of investment management. Slager has experienced that applying investment theory is not self-evident in practice, for example the optimization procedure for risk and return as proposed by Markowitz $(1952,1959)$ is not an effective tool because the data input can lead to non-sensical portfolios. Another example is that the results of active management, the pursuit to outperform a benchmark, are doubtful. In general, investment theory needs interpretation to be put to use fruitfully in practice. The interpretation of investment theory is also a part of the investment beliefs.

Dutch pension funds have shown interest in working with investments beliefs. Slager has advised some 30 Dutch pension funds in articulating their investment beliefs, some more in-depth than others. For a thorough review, boards of pension 
funds need to invest time to discuss their investment beliefs, because, again, the beliefs often are implicit. Of the 30 pension funds, two-thirds ended up in successfully describing their investment beliefs, the other third did not fully pursue the process of investigating their beliefs.

\section{Beliefs about Capital Markets}

The beliefs about capital markets can be clustered in four themes: risk premiums, diversification, market inefficiencies and the investment horizon (Koedijk \& Slager, 2011). I will not treat the themes in depth here because the themes have been treated at length in the dissertation. The existence of a risk premium of stocks and other investments is a basic belief for investors. According to Slager, not to believe in the risk premium would be to disbelief the future success of capitalism, which has achieved economic growth by achieving a higher return than risk free investments by taking risk. Market efficiencies are beliefs about beating the market index, a subject which has been treated in the dissertation in Chapter 2. Koedijk \& Slager (2011, p. 79) advise boards of pension funds to be careful about active investment strategies because there no clear proof that such strategies deliver persistent excess returns after costs. Koedijk \& Slager (2011, p. 102) stress that diversification may not work in a time of crisis on the financial markets, and advise to look at the economic ratio of new asset categories if they are meant for diversification. The subject of diversification has been treated in the dissertation in Chapter 2. The fourth aspect of the investment horizon is about the flawed notion that a longer horizon diminishes investment risk. The investment horizon and its pitfalls have also been a subject of Chapter 2 of the dissertation in the appendix. Koedijk and Slager apply an evidence-based approach here; it is not their individual investment opinion that guides these proposals, but rather the state of empirical research, seen through the lens of a decision maker of a pension fund.

\section{Beliefs about the Organization of Investments}

The organizational beliefs about investments matter for governance. Concerning the investment beliefs about governance, the principal-agent problem in investment 
management often is overlooked. The agents, the asset managers, have other interests other than the principal, the board of the pension fund, and ultimately the participants. The asset managers pursue their ideas as investors. The principal, the board of the pension fund generally lack detailed or expert insight into investment management, also because it takes a number of years to become aware and to understand what investing is about, and by then board members may have left or changed roles. Key is that the governance investment beliefs are formulated according to the interests of the participants. Slager \& Koedijk (2007) refer for the theory of governmental beliefs to the agency theory of Jensen \& Meckling (1976). Awareness of agency problems should lead to minimizing agency costs, which consist of the monitoring costs of the principal, the bonding cost that the agent makes to demonstrate that the agent works in the interest of the principal, and the residual loss, the cost that remains because of the imperfection of the monitoring of the principal.

The societal beliefs concern values such as the environment and human rights which are to be considered by Dutch pension funds in their investment policy. An oversight of the practices of responsible investing in the Netherlands can be found in Koedijk \& Slager (2011, pp. 190-204).

\section{What Should Be Done about the Current State of Finance?}

A key question is how the theory and practice of finance can be changed. Besides engaging agency theory in practice as discussed, the practical side of behavioral finance can be put to use in investment practice. Slager refers to Gigerenzer (1999 with Todd, 2000) as an author on behavioural finance who has practical relevance (see also Chapter 2 of the dissertation in which the ideas of Gigerenzer are treated).

Another theme which is underexposed in finance and economics is that economics is also political economics. Economics is not merely a technical subject, but also deals with values such as fairness and the distribution of wealth. Analogous to economics, finance is not seen as a political subject, but nevertheless has ethical 
value aspects: money is not neutral and influences people as it potentially can make them greedy, negatively affecting the alignment between principal and agent. This phenomenon is generally attributed to the financial sector at large. However, this occurs at all levels; within boards, between boards and their executive offices, as well as between the pension fund and asset manager. Here too, the formulation of beliefs (and the organizational embedding) provides a self-disciplining mechanism to potentially counter these effects. The depoliticization of finance has been a subject in the dissertation in the current Chapter.

Despite that Slager would like to see that more attention to ethics would change behaviour of the organizations and professionals involved in investing, he does not expect that ethics is the solution to improve the current state of finance. However, he posits that the sector should not be afraid to make fiduciary and ethical norms more explicit than is the case today. Rather, the focus in the Netherlands is on making the pension contract explicit, but this does not induce change. As an example he refers to the effects of the ERISA laws of the United States. ERISA is the abbreviation of 'Employee Retirement Income Security Act' of 1974) and is meant to protect participants of pension scheme by setting standards of, and disclosure for, conduct of pension funds in the United States. ERISA forces for example a constant urge to investors to explain why investments are made in the interest of the participants, and sharpens trustee's minds on what really matters in the principal-agent dilemma's that they might face.

To change the practice of finance, Slager does not strongly believe that changing the finance education would change the practice. First, it would be difficult to fundamentally change the finance education because the received view of finance dominates strongly and would basically require a whole new generation of tutors, textbooks and method of teaching. Second, there is the alluring career perspective to consider. Students of finance simply find active management more appealing than passive investment as advocated by the theory of efficient markets. Ending on a more positive note, placing more focus on the institutional and ethical context of 
investment discussion will help change the mindset, yet slowly. Also a form of belief, in Slager's view.

\section{9: Values, Conversations, their Justification, and Innovation}

\section{The Values of Investors}

Klamer regards values as an important aspect of culture. The values of a culture are not evident and therefore have to be interpreted. Emerging from my personal observations, the interviews, the literature, and the dissertation, in my view, the prime shared values of investors are wealth, optimism, and rationalism.

\section{Wealth}

The investment of capital in the financial markets aims to earn money for future income or consumption, or to preserve capital. An intriguing point about the culture of investing is that wealth, money, cannot actually be an end, because money is always instrumental to achieve something else (Klamer, 2014, p. 9). Values are about what is important in life, the 'things' we strive for. Klamer uses the oikos, the home, as an example of a value which will probably be important to most of us. So, we have to understand the values of the people for whom is invested. Indeed, institutional investors must be interested in the values of their clients, also because investing is an instrument with uncertain outcomes. Accepting the uncertain outcomes of investing as an instrument is a leap of faith.

\section{Optimism}

The public expects stocks to deliver a positive return above bonds in the long run. The optimism in the culture of investing roots in our liberal, free-market society. The free-market system has realized a remarkable high economic growth (McCloskey, 2010). Economic growth and profits are related: in general higher economic growth means higher profits. And, higher profits mean in general higher prices of stocks. In Chapter 4, I have argumented that the transfer mechanism of 
economic growth (not a sure thing either) to stock prices is more complicated and uncertain than explained here.

The level of optimism about the stock market varies. In a boom time the optimism is at its highest level and shared by professional investors and the investing public alike: investing then becomes a part of the popular culture. The optimism of investors in a boom, makes being negative on the outlook of the stock market a taboo, because people are too eager to become rich. Probably therefore, no forecaster is easily held accountable for his optimism. I cannot recall investors in the Netherlands who lost their job after the credit crisis of 2008, because they were accused of too much optimism. An exception of a person held accountable was, as told in Chapter 2, Irving Fisher, who is even nowadays remembered for his unfortunate optimistic prediction in October 1929 before the crash. As a matter of culture, the optimistic sayings of experts are part of the ritual of investing. In times of bust, professional investors will maintain their optimism. Professional investors who doubt on the optimism will have a hard time functioning in the culture of investing and probably will pursue another career. So, self-selection of professional investors ensures a continuing optimism.

My observation has been that the optimism about investing is both present in commercial and non-commercial institutions. A commercial setting can however stimulate investors to be more optimistic because doubt does not persuade clients. Also will personal character traits encourage some professional investors, though not the majority, to leverage their beliefs.

\section{Rationalism}

Handling uncertainty, a feeling of anxiety, has since long been a universal part of culture (Hofstede et al, 2007). Using a predictive method handles uncertainty by replacing a feeling of uncertainty with a rationalistic method. Since the $1860 \mathrm{~s}$, vernacular science has modelled investing after engineering, and has promoted 
rational behaviour as the role model for investors (Preda, 2006). Rational behaviour consists of self-control and the study of information. Vernacular science results in the efficient market theory and the use of the statistical approach as explained at length in Chapter 2. The analysis of the rhetoric of investment theory has also demonstrated that modernism is form of rationalism that has heavily influenced current investment theory and practice. Predictionism is a form of modernism and expresses itself in investment theory in the form of teleological equilibrium thinking, valuation theory, and stochastical predictability.

\section{The Conversations of Investors}

Besides values, Klamer has proposed to investigate conversations as an utterance of culture. In my view, the archetypical conversations of investors, again emerging from my personal observations, the interviews, the literature, and the dissertation, are about 'The market and the economy', 'Talk by the model', 'Money must be put to work' and 'Doubt and reassurance'. The archetypical conversations can, of course, be combined.

\section{The Market and the Economy}

As an investor you have to be informed about the news, so the media, views of brokers, and the development on the markets are important. Investors talk a lot about developments on the markets and the economy, and interpret the development in order to be able to make decisions. Though the media look like objective observers, they spread the ideas about the markets. Brokers do effectively the same thing as media.

\section{Talk by the Model}

As often in complex phenomena, a rationalisation is needed to get in control. It is the same for financial markets. For example quantitative models based on investment theory supply information, input, to the conversations and decisions of investors. The models make a conversation possible about risk and return of investments, or the selection of stocks. Models also legitimise investment 
decisions, for example: 'If we enlarge our stake in stocks with $10 \%$ our risk return profile will benefit'.

\section{Money Must Be Put to Work}

An argument heard often argument in the conversations about investing is that money on a savings account should be replaced by investments, because money should, instead of doing nothing, be activated in the financial market. De Goede (2005) also discusses this theme. The same kind of discussions take for example place about bonds versus stocks or other securities. The discussions about putting money to work tend to be about taking more risk, and are often based on optimism.

\section{Doubt and Reassurance}

A discourse such as about investing has to be repeated frequently. The cycle of doubt and reassurance fits in the discourse as Shiller (2000) has demonstrated by his analysis of interviewers of the media and investment professionals. The interviewer asks critical questions about the financial markets, and the investment professionals reassures the interviewer. The interview ends with a supportive view of the market. The same kind of conversations about the markets, specific investments, or investment strategies take place between a client and an investment expert or advisor. Obviously, the conversation of doubt and reassurance is needed because of the uncertainty of investing.

\section{The Justification of the Culture of Investing}

How to relate Klamer's investigation of the culture of economics to that of the culture of investing? The culture of economics is about getting attention. Instead of on attention, the culture of investing concentrates on wealth. Conceptually, the legitimization of a conversation can consist of three parts: a transcendental justification (religion or truth), a social justification (the meaning for society), and a personal justification (such as the need to make a living) (Klamer, 2007, pp. 4748). Getting attention in economics is a social justification. To consider truth as the only effective contribution, would reflect the philosophical realist position: for 
short, the realist position expects a theory to conform to the external world, and so to be true in the notion of truth as correspondence to the external world. But effective contributions can also be established if they are meaningful or interesting, for example because a contribution aids to understanding. A contribution can also be pragmatic, for example by giving guidelines for what to do. Yet, in the end, the contribution of a conversation has to be effective. The conversation must be able to sensibly answer the 'So what?' question.

For investing, the 'So what?' question is whether the investments lead to more wealth. McCloskey puts the forecasting abilities of investor to the test by asking the 'So what?' question in the form of the American question: "If You're So Smart Why Ain't You Rich? (1990)." The answer to the question is one of common sense: investors earn a living by 'selling' their predictions, but cannot really predict. The social justification of investing is to make money by investing to provide future income or wealth to the owners. The personal justification for investors is that they make a living out of making money for their clients, and probably that they like or need their job. Investing has a transcendental justification as well, investing is nowadays a matter of unquestioned faith (De Goede, 2005). Like economics, finance is depoliticized and viewed as a technical subject, not a moral subject.

What about truth as a justification for investing? Investors need theories to help them act, but are the theories really true, or are they to be viewed as practical guides? In Chapter 4 the scientific context of modernism was treated. Modernism has moulded investment theory into a stochastical predictive framework. Modernism is a part of the background culture of investment theory and is a possible explanation for the depoliticization of finance. The denial of the normative aspect in modernist science intents to keep science confined to truth. In finance, a statistical formulation leads to the idea that the applied theory of statistics is meaningful. But an improper theory of statistics fools one into the 
feeling of certainty, and does not explain nor predict financial markets. Of course, efficient markets are an interesting notion of modern finance, just like diversification and value: theories have a worth for themselves, but does that make them a good guide for practice? In practice, the ideas of finance together with other ideas serve as a practical guide, like engineering serves to build a bridge. But investing is not like engineering, because the analogy of economics as mechanical physics does not hold (Knight, 1921).

\section{Evaluation of Innovative Practices}

An interesting outcome of investigating practice is that practice combines various theoretical and practical viewpoints as a matter of phronesis, as foreseen by Klamer. Kocken uses his insight gained as a risk manager that VaR-models based on portfolio theory do not function, to implement stress testing as the alternative. Stress testing is not based on statistics. Furthermore, he combines the insight of Minsky's instability hypothesis, a non-mainstream macro-economic theory, as an argument for better risk management by stress testing. To understand why risk and investment professionals stick to the current investment theory, he applies insights from behavioural finance. He also advocates the bottom-up approach of evolutionary finance. Concerning investment theory, he advocates the use of option theory. Options make sure that downside risk is covered.

Slager combines insights from the practice of investing with management and agency theory. He asks a Socratic question when he asks investors what beliefs they have about investing, and takes uncertainty as a starting point. Next, he emphasizes that organizational aspects are important in investing, that management matters. He also underpins the agency problem for pension funds, which in essence reflects the metaphor of investing as power relations between the participants, the board of the pension fund, and the investment managers.

The Shell scenarios approach applied to investing by Heijmans, is of a nonstochastical character. Mechanical statistical forecasting has not been effective in 
the past for the business of Shell and is regarded as an enemy to thinking. In essence, the scenario approach is about the social construction of a potential future, founded on uncertainty, conversation, and practical wisdom. The scenario approach has emerged from management practice, the management of uncertainty.

Kocken and Heijmans share the conviction that scenario thinking is a better alternative for investing than applying statistical models. But both acknowledge that statistical models have a specific benefit as well. In my words, statistical models for investing reveal how stakeholders of a pension fund or parties in an option contract divide risk and return: in essence, the models try to quantify statistically the equity, the fairness of the distribution of risk and return between parties involved. The quantification serves to make the fairness between stakeholders or parties visible, and is not meant, or should be understood, as a specific prediction. Yet, the model is useful, because it provides an insight which would otherwise not have been available.

Many ideas and practices exist to change the theory and practice of finance. Besides entrepreneurship in the financial services, changing education is important to Kocken. Therefore he has launched a MSc in Risk Management with a multidisciplinary approach. He also tries to reach a wider audience for the failing of economics and finance with the documentary Boom Bust Boom. Furthermore he supports student organizations that demand more pluralist education in economics. Concerning science, he suggests that economics and finance should investigate the complexity of economic and financial markets phenomena with a bottom-up approach. Slager suggest that, besides agency theory, the practical side of behavioral finance can be put to use in investment practice by the ideas of Gigerenzer. Further he suggest that finance is to be regarded as a political subject, because money is not neutral. Slager expects that laws could change the behaviour of investors. As an example he refers to the effects of the ERISA laws of the United States. 
In the dissertation, other ideas to change finance have had attention as well: the alternative investment theory with the statistical theory of Mandelbrot, bubble theory, evolutionary finance, political finance, the analysis of rhetoric, the analysis of culture, ethics in the form of virtue and value ethics, and financial history. A more heterogeneous culture of academic finance and investment practice could accommodate the ideas mentioned: the alternatives for modern investment theory are plenty. How to make the culture of finance more heterogeneous? That starts with awareness of the current problems, the introduction of multiform metaphors for investment theory, and handling the ambiguity of theory uncertainty, that is having competing theories of finance, by presenting, explaining and elaborating the alternatives. 


\section{Chapter 6}

\section{Conclusions}

Mainstream investment theory assumes stochastical prediction. Stochastical prediction is a special apodictic form of prediction, which makes the characteristics of a group of outcomes predictable, like the outcomes of throwing a dice. In the dissertation the central question was: What grounds the use of stochastical predictability in investment theory? The question has no simple answer because the assumption is implicit in investment theory and backed by statistical theories, of which the assumptions are implicit as well. To get a complete answer to the question of the foundations of stochastical predictability in investment theory, the dissertation used the form of a rhetorical analysis, enriched by uncertainty economics, philosophy of statistics, history, culture, innovative practices, and literature on alternative investment theories. The purpose of the dissertation is that it should result in a refutation of stochastical predictability in investment theory.

We will now look at the answers to the subquestions of the dissertation:

\section{What is the rhetoric of investment theory?}

The analysis of the rhetoric of investment theory in the dissertation is grounded on the approach of the rhetoric of economics by McCloskey. Therefore, the rhetoric of investment theory is, among other things, about the discourse and metaphors (constitutional ideas) of investment theory. The history of investment theory provides a good starting point for a discussion of its discourse. Since the 1960s, finance and neoclassical economics belong together, which the name financial economics demonstrates. Yet, the notion that investment theory is a young science and merely has existed since the 1960 s is flawed. Finance as relevant for investment theory has existed since the $13^{\text {th }}$ century and is grounded on actuarial 
science and probability theory. The legitimization in the 1860 s of investing in stocks, which until the $19^{\text {th }}$ century were associated with gambling, is done by vernacular science which results in efficient market theories, because it included probabilistic and abstract reasoning.

Another part of the discourse of investment theory stems from McCloskey's analysis of the rhetoric of economics. After all, investment theory belongs to economics since the 1960s. McCloskey's discourse analysis considers economics as a product of the modernistic scientific culture of logical positivism, which has led to a dogmatic emphasis on prediction, mathematics, and statistical significance testing.

To complete the discourse of investment theory with other findings in the dissertation, investment theory is characterized by stochastical equilibrium theories. Markowitz's portfolio theory uses the theory of valuation which assumes that the price of a security will tend to its intrinsic, equilibrium, value. In the Capital Asset Pricing Model, the continuation of Markowitz's portfolio theory, informational efficiency ensures an equilibrium of risk and return.

The main metaphor in Markowitz's investment theory is the claim that investment management is mathematical statistics, in which investment returns are a probability distribution. The application of mathematical statistics assumes calculability and distracts the attention from uncertainty. The second metaphor in Markowitz's theory is the machine, which can be recognized in the language of, and aspiration to efficiency. The machine metaphor focusses on the process parts of reaching efficiency, such as the efficiency criteria and the algorithms, and beliefs are treated merely as inputs for the calculating machine.

The dominant metaphor in the CAPM is the equilibrium: the market of investments is in balance because of informational efficiency and rational expectations. In equilibrium, risk and return have become predictable. Equilibrium and predictability are exchangeable. The second important metaphor is the 
informational efficiency. It is again a machine metaphor, but now concerning the processing of information. Because of informational efficiency the market of investments is at any time in balance: new information is immediately being processed into a new equilibrium.

\section{What philosophy of statistics is applied in investment theory?}

The arguments for assuming stochastical predictability are founded on a theory of statistics. The various theories of statistics have implicit assumptions about the structure of reality, in other words, the theories of statistics have different philosophies of probability. Markowitz proposes to use subjective, personal probabilities for decision making with his portfolio theory. Markowitz's theory is grounded on Savage's personal probability theory in which the reliability of the probability beliefs is irrelevant. The theory of objective probabilities, aka the frequency theory, founds the CAPM. The ergodic assumption as used in the rational expectation hypothesis in the CAPM assumes that objective probability information is available from past data.

\section{What are the arguments for uncertainty as the opposite of predictability?} Non-mainstream economics assumes unpredictability instead of predictability and has heavily debated predictability and statistics. Their arguments against predictability and statistics are an important source for making the case against predictability. Knight calls stochastical predictability 'risk': a situation under risk results in certainty when a 'whole' group of cases is available. He calls a situation without stochastical predictability 'uncertain'. Economic and investing phenomena float in between risk and irreducible uncertainty, and are called 'uncertain'. Uncertainty means that the probabilities are unmeasurable. Keynes relates financial markets explicitly to uncertainty, and invents an investment theory upon rational expectations under uncertainty. Furthermore, he introduces the role of animal spirits in economic phenomena, which contrast rationality. Von Mises's argument for uncertainty is first that intent human action features teleological 
causality, which cannot be predicted, and second that natural sciences do not predict the world as a whole either, so why claim predictability for economics? McCloskey refutes uncertainty by the absence of profitable predictability: if forecasting is easy, economists would get rich by exploiting their knowledge. Taleb's argument for uncertainty is that black swans determine risk and returns of investments; but one cannot predict black swans.

I have also presented a thought experiment about the assumption of stochastical predictability in investment theory. The experiment puts the statistical outcomes of modern investment theory to the test of risk-free arbitrage, that is the other dominant branch of investment theory, which implies, in contrast to modern portfolio theory, certainty of outcomes. The thought experiment uses the paradigm of risk-free arbitrage of finance to illustrate that the paradigm of predictability of finance is flawed.

\section{Do alternative investment theories offer a better explanation, modelling, prediction or handling method?}

Modern investment theory has interesting rivals, which have in common that they do not pretend to predict financial markets. The alternative theories aim at explanation or modelling of financial markets. The ideological criticism of modern finance, political finance, uses elements of behavioural and bubble criticisms, and combines them with the criticism on free markets in general. Mandelbrot's fractal finance provides within the rational mathematical tradition an alternative statistical theory, which models seemingly predictive patterns in financial markets, wild volatility, and bubbles. The bubble theory explains how bubbles in (financial) markets arise. Behavioural finance is insightful and helps to reflect on decision making, and explains behaviour of market participants. The bottom-up approach of evolutionary finance yields an interesting alternative to the top-down approach of modern investment theory. Concerning the depth of the rival theories of finance, they all have substantial backing and empirical proofs which can compete scientifically with modern investment theory. 
5. Can virtue and value ethics compensate the assumed epistemological deficiencies of investment theory in decision making?

If the epistemology of mainstream and alternative investment theories is not suited for predicting, practical reason in the form of virtue or value ethics can become relevant, also because the ethics in economics and investment theory is reduced to merely the rationality of prudence. Both McCloskey's virtue approach and Klamer's value approach can compensate the lack in epistemological strength of modern investment theory. McCloskey's approach tries to ensure a balanced approach by reminding us of all the seven virtues, Klamer's approach is a way of sense making for individuals at home or in an organisation, and is embedded in the virtues that McCloskey proposes.

6. What explanations offers an investigation of the culture of investing for the use of investment theory?

As an extension of rhetoric, the perspective of Klamer's approach of the culture of economics is relevant and applicable to investing, because culture is extension of rhetoric and relates to uncertainty. Uncertainty, a feeling of anxiety, is handled by culture in numerous ways. In Klamer's approach of culture, values and conversations are central. In my view, the prime shared values of Dutch institutional investors are:

- Wealth: the investment of capital in the financial markets aims to earn money for future income or consumption, or to preserve capital. A problem here is that money is never an end, but a means.

- Optimism: the optimistic sayings of investment experts are part of the ritual of investing. Even in times of bust, professional investors will maintain their optimism. 
- Rationalism: Using a predictive method handles uncertainty by replacing a feeling of uncertainty with a rationalistic method. Rational behaviour further consists of self-control and the study of information.

In my view, the archetypical conversations of investors are about 'The market and the economy', 'Talk by the model', 'Money must be put to work' and 'Doubt and reassurance'.

\section{Purpose of the dissertation}

The purpose of the dissertation was to investigate stochastical predictability in investment theory. The investigation of stochastical predictability is relevant for science, but also for investors, the financial sector, policymakers, bankers, and the society at large, because treating unpredictability as predictability can and did harm the economy and people's wealth. The proof of unpredictability lies outside the paradigm of statistics, because statistics assumes that the substrate that produces probability outcomes is stable. The inference to the best explanation that investment returns are unpredictable, based on the extensive reflection in the dissertation, seems more persuasive than the arguments of investment theory that personal or objective probability beliefs predict.

The new proposed rhetoric, discourse, for investment theory is that investing takes place under Knightian uncertainty, which makes statistics supportive rather than dominant in decision making. So, investment theory should become more a moral science and less an engineering one: it is about judgments, combining virtue and value ethics with historical and theoretical insights, also from alternative investment theories, $a k a$ heterodox finance, which can enrich mainstream investment theory and guide practice. An interesting outcome of the investigation of a number of innovative investment cases in practice is that innovative practice combines various theoretical and practical viewpoints as a matter of practical 
wisdom, to mention a few: stress testing, the use of option theory to reduce risk, management theory, agency theory, or a scenarios approach. 


\section{Works Cited}

Anufriev, M., Hommes, C.H, \& Philipse, R.H.S. (2013), "Evolutionary selection of expectations in positive and negative feedback markets", Journal of Evolutionary Economics, Vol. 23 No. 3, pp. 663-688.

Aristotle (1908), Nicomachean Ethics, translated by W.D. Ross in 1908, available at http://classics.mit.edu/Aristotle/nicomachaen.html (accessed on 11 May 2013).

Aristotle (1912), Politics: A Treatise on Government, translated by A.M. William Ellis, Dent \& Sons Ltd, London \& Toronto, available at http://www.gutenberg.org/files/6762/6762-h/6762-h.htm (accessed on 20 September 2015).

Aristotle (2006), The 'Art' of Rhetoric, translated by J.H. Freese in 1926, Harvard University Press, Cambridge, MA.

Atkinson, T., Luttrell, D. \& Rosenblum, H. (2013), "How Bad Was It? The Costs and Consequences of the 2007-09 Financial Crisis", Staff Papers Federal Reserve Bank of Dallas, Vol. 20 No. 1, pp. 1-22.

Audi, R. (ed.) (1995), The Cambridge Dictionary of Philosophy, 2nd ed. 1999, 11th printing 2006, Cambridge University Press, Cambridge.

Bachelier, L.J.A. (1900), Théorie de la speculation (The Theorie of Speculation), Annales scientifiques de l'École Normale Supérieure, Sér. 3, 17, pp. 21-86, translated D. May in 2011, available at http://www.radio.goldseek.com/ bachelier-thesis-theory-of-speculation-en.pdf (accessed on 16 november 2014). 
Beinhocker, E.D. (2006), The Origin of Wealth: Evolution, Complexity, and the Radical Remaking of Economics, original edition by Harvard Business School Press, edition 2007 by Random House, London.

Bernstein, P.L. (1992), Capital Ideas: The Improbable Origins of Modern Wall Street, originally published by Free Press, New York, edition 2005, John Wiley \& Sons, Hoboken, N.J.

Bernstein, P.L. (1996), Against the Gods: The Remarkable Story of Risk, John Wiley \& Sons, New York.

Bernstein, P.L. (2007), Capital Ideas Evolving, John Wiley \& Sons, Hoboken, N.J.

Bernstein, P.L. \& Damodaran, A. (eds.) (1998), Investment Management, John Wiley \& Sons, New York.

Black, F. (1986),"Noise", Journal of Finance, Vol. 41 No. 3, pp. 529-543.

Black, F. \& Scholes, M. (1973), "The Pricing of Options and Corporate Liabilities", Journal of Political Economy, Vol. 81 No. 3, pp. 637-654.

Blackburn, S. (ed.) (2008), The Oxford Dictionary of Philosophy, $2^{\text {nd }}$ revised edition online, Oxford University Press, available at www.oxfordreference.com (accessed on 18 September 2015).

Bloom, M.T. (1974), Rogues to Riches, Warner Books, New York.

Bodie, Z., Kane, A., \& Marcus, A.J. (1989), Investments, 3rd edition, 1996, IRWIN, Chicago.

Bodie, Z. (1995), "On the Risk of Stocks in the Long Run", Financial Analysts Journal, Vol. 51 No. 3, pp. 18-22.

Bryman, A. \& Bell, E. (2003), Business Research Methods, $3^{\text {rd }}$ edition 2011, Oxford University Press, Oxford. 
Cardim De Carvalho, F.J. (1988), "Keynes on Probability, Uncertainty, and Decision Making", Journal of Post Keynesian Economics, Vol. 11 No. 1, pp. 66-81.

Cardano Company (2015), “Company information” available at https://www.cardano.com/nl-nl/cardano-van-dichtbij (accessed on 22 May 2015).

Cardoso, J.L. (2006) Joseph de la Vega and the Confusion de confusiones, in Poitras, G. (ed.) (2006), Pioneers of Financial Economics, Volume 1: Contributions Prior to Irving Fisher, Edward Elgar, Cheltenham.

Cassidy, J. (2009), How Markets Fail: the Logic of Economic Calamities, Penguin Books, London, edition 2010, first published by Farrar, Straus \& Giroux, United States.

CFA Society Netherlands, "CFA Society Netherlands Annual Report 2013/2014", available at http://www.cfasociety.org/netherlands/Documents/

CFA\%20NL\%20Annual\%20Report\%202013-2014\%20\%28final\%29.pdf (accessed on 15 September 2015).

Chuquet, N. (1484), Triparty en la science des nombres, edition 1881, Imprimerie des Sciences Mathématiques et Physiques, Rome, available at 12 October 2014, http://gallica.bnf.fr/ark: /12148/bpt6k62599266/f64.image.r=.langFR (accessed on 12 October 2014).

Cicero (2001), De Oratore (On the Ideal Orator), translated by J.M. May \& J. Wisse, Oxford University Press, New York.

Collins, H.M. \& Evans, R. (2007), Rethinking Expertise, paperback edition 2009, University of Chicago Press, Chicago.

Cottrell, A. (1993), "Keynes's Theory of Probability and its Relevance to his Economics", Economics and Philosophy, Vol. 9 No. 1, pp. 25-51. 
Cowles 3rd, A. (1933), “Can Stock Market Forecasters Forecast?”, Econometrica, Vol. 1 No. 3, pp. 309-324.

Cowles 3rd, A. (1944), "Stock Market Forecasting”, Econometrica, Vol. 12 No. 3/4, pp. 206-214.

Davidson, P. (1982/1983), "Rational Expectations: A Fallacious Foundation for Studying Crucial Decision-Making Processes", Journal of Post Keynesian Economics, Vol. 5 No. 2, pp. 182-198.

Davidson, P. (1991), "Is Probability Theory Relevant for Uncertainty? A Post Keynesian Perspective", Journal of Economic Perspectives, Vol. 5 No. 1, pp. 129-143.

Davidson, P. (2009), "Risk and Uncertainty in Economics", paper presented at the conference on "The Economic Recession and the State of Economics", Westminster, London, 6 February, available at http://econ.bus.utk.edu/davidsonpapers/riskanduncertaintyineconomics.pdf (accessed on 15 September 2013).

Davidson, P. (2010), “Black swans and Knight's epistemological uncertainty: are these concepts also underlying behavioral and post-Walrasian theory?", Journal of Post Keynesian Economics, Vol. 32, No. 4, pp. 567-570.

De Finetti, B. (1940), Il problema dei 'Pieni', in Giornale dell' Istituto Italiano degli Attuari 11, pp. 1-88, translated as 'The problem of full-risk insurances, Chapter I The risk within a single accounting period, translated by L. Barone in 2006, Journal of Investment Management, Vol. 4 No. 3, pp. 19-43.

De Goede, M. (2005), Virtue, Fortune, and Faith: A Genealogy of Finance, University of Minnesota Press, Minneapolis and London. 
De la Vega, J. (1688), Confusion de Confusiones, edition 1939 by M.F.J. Smith, Martinus Nijhoff, Den Haag.

De Nederlandsche Bank (2014), "Tabel vermogenscomponenten van Nederlandse huishoudens", translated as "Table Capital Components of Dutch Households", available at http://www.statistics.dnb.nl/huishoudens /index.jsp (accessed on 7 April 2015).

Dequech, D. (2000), "Fundamental Uncertainty and Ambiguity”, Eastern Economic Journal, Vol. 26 No. 1, pp. 41-60.

Derman, E. \& Taleb, N.N. (2005), “The Illusions of Dynamic Replication”, Quantitative Finance, Vol. 5 No. 4, pp. 323-326.

Dimand, R.W. (2007), Irving Fisher and his students as financial economists, in Poitras, G. (ed.) (2007), Pioneers of Financial Economics, Volume 2: Twentieth-Century Contributions, Edward Elgar, Cheltenham.

Dimson, E., Marsh, P., Staunton, M. et al (2012), "Credit Suisse Global Investment Returns Yearbook 2012", available at https://www.creditsuisse.com/investment_banking/doc/cs_global_investment_returns_yearbo ok.pdf (accessed on 15 June 2013).

Durant, D. (1957), "Growth Stocks and the Petersburg Paradox", The Journal of Finance, Vol. 12 No. 3, pp. 348-363.

Durlauf, S.N \& Blume, L.E. (eds.) (2008), The New Palgrave Dictionary of Economics, 2nd edition, available at http://www.dictionaryofeconomics.com/ (accessed on 1 november 2014).

Ellsberg, D. (1961), "Risk, Ambiguity, and the Savage Axioms", Quarterly Journal of Economics, Vol. 75 No. 4, pp. 643-669. 
Fama, E.F. (1963), "Mandelbrot and the Stable Paretian Hypothesis", The Journal of Business, Vol. 36 No. 4, pp. 420-429.

Fama, E.F. (1965a), "Random Walks in Stock Market Prices", Financial Analyst Journal, Vol. 21 No. 5, pp. 55-59.

Fama, E.F. (1965b), "Behavior of Stock-market prices", The Journal of Business, Vol. 38 No. 1, pp. 34-105.

Fama, E.F. (1970), "Efficient Capital Markets: a Review of Theory and Empirical Work", The Journal of Finance, Vol. 25 No. 2, pp. 383-417.

Fama, E.F. (2011), "My Life in Finance”, Annual Review of Financial Economics, Vol. 3 No. 1, pp. 1-15.

Fama, E.F. \& French, K.R. (1993). "Common risk factors in the returns on stocks and bonds", Journal of Financial Economics, Vol. 33 No. 3, pp. 3-56.

Fetterman, D.M. (2015), “Ethnography in Applied Social Research”, International Encyclopedia of the Social \& Behavioral Sciences, 2nd edition, edited by J.D. Wright, Elsevier, Oxford, pp. 184-191, available at http://www.sciencedirect.com/science/article/pii/B9780080970868105082 (accessed on 15 September 2015).

Feyerabend, P. (1975), Against Method, 4th edition 2010, Verso, London.

Fisher, I. (1930), The Theory of Interest, as determined by Impatience to Spend Income and Opportunity to Invest it, Macmillan, New York, available at http://files.libertyfund.org/files /1416/Fisher_0219.pdf (accessed on 10 March 2015).

Fox, J. (2009), The Myth of the Rational Market: A History of Risk, Reward, and Delusion on Wall Street, HarperCollins Publishers, New York. 
Friedman, M. (1953), “The Methodology of Positive Economics”, pp. 3-43, in his Essays in Positive Economics, 1966, Chicago University Press, Chicago.

Galbraith, J.K. (1954), The Great Crash 1929: The Classic Study of that Disaster, revised edition of 1975, reprinted by Penguin Books in 1992, Penguin Group, London.

Galbraith, J.K. (1977), The Age of Uncertainty: The Age of Uncertainty Episode 1 The Prophets and Promise of Classical Capitalism, available at https://www.youtube.com/watch?v=KGSID_Uyw7w (accessed on 10 May 2015).

Galbraith, J.K. (2001), The Essential Galbraith, Houghton Mifflin Company, Boston and New York.

Gigerenzer, G., Todd, P.M. \& the ABC Research Group (1999), Simple Heuristics That Make Us Smart, Oxford University Press, New York.

Gigerenzer, G. (2000), Adaptive Thinking: Rationality in the Real World, Oxford University Press, New York.

Gilboa, I. (2009), Theory of Decision under Uncertainty, Cambridge University Press, Cambridge.

Gilboa, I., Postlewaite, A.W., \& Schmeidler, D. (2008), "Probability and Uncertainty in Economic Modelling", Journal of Economic Perspectives, Vol. 22 No. 3, pp. 173-188.

Goetzmann, W.N. (2005), Fibonacci and the Financial Revolution, in W.N. Goetzmann \& K.G. Rouwenhorst (eds.) (2005), The Origins of Value: The Financial Innovations That Created Modern Capital Markets, Oxford University Press, Oxford. 
Goetzmann, W.N. \& Rouwenhorst K.G. (eds.) (2005), The Origins of Value: The Financial Innovations That Created Modern Capital Markets, Oxford University Press, Oxford.

Graeber, D. (2011), Debt: the First 5,000 Years, paperback edition 2012, Melville House Publishing, Brooklyn.

Graham, B., Dodd, D.L., \& Cottle, S. (1934), Security Analysis, 4th edition, 1962, McGraw-Hill, New York.

Griswold, C.L. (2012), "Plato on Rhetoric and Poetry", Stanford Encyclopdia of Philosophy, available at http://plato.stanford.edu /entries/plato-rhetoric/ (accessed on 10 August 2012).

Gunderson, E. (2009), The Cambridge Companion to Ancient Rhetoric, Cambridge University Press, Cambridge.

Hacking, I. (2001), An Introduction to Probability and Inductive Logic, edition 2009, Cambridge University Press, New York.

Hammersley, M. \& Atkinson, P. (1983), Ethnography: Principles in Practice, Tavistock Publications, London and New York.

Hansen, P.L. \& Sargent, T.J. (2008), Robustness, Princeton University Press, Princeton.

Haug, E.G. \& Taleb, N.N. (2011), “Option traders use (very) sophisticated heuristics, never the Black-Scholes-Merton formula", Journal of Economic Behavior \& Organization, Vol. 77 No. 2, pp. 97-106.

Haugen, R.A. (1995), The New Finance: The Case Against Efficient Markets, Prentice-Hall, N.J. 
Hausman, D.M. (1981), Capital, Profits, and Prices: An Essay in the Philosophy of Economics, Columbia University Press, New York.

Hausman, D.M. (1989), "Economic Methodology in a Nutshell”, Journal of Economic Perspectives, Vol. 3 No. 2, pp. 115-127.

Hausman, D.M. \& McPherson, M.S. (1988), "Standards", Economics and Philosophy, Vol. 4 No. 1, pp. 1-7.

Hayek, F.A. (1948), Individualism and Economic Order, paperback edition 1980, University of Chicago Press, Chicago.

Heilbroner, R.L. (1996), Teachings from the Worldly Philosophy, paperback edition 1997, W.W. Norton \& Company, New York.

Heijmans, P. (2014), "Shell SCENARIOS", presentation given at 6 November 2014 at the Asset Liability Management Congress of the Verening van Beleggingsanalisten in Amsterdam, Shell Asset Management Company.

Ho, K. (2009), Liquidated: An Ethnography of Wall Street, Duke University Press, Durham and London.

Hofstadter, D.R. (1979), Gödel, Escher, Bach: an Eternal Golden Braid, Basic Books, New York.

Hofstede G.H., Hofstede G.J. \& Minkov, M. (1997), Cultures and Organizations: Software of the Mind: Intercultural Cooperation and Its Importance for Survival, $3^{\text {rd }}$ edition of 2010, McGraw Hill, New York.

Hollis, M. (1985), “The Emperor's Newest Clothes”, Economics and Philosophy, Vol. 1 No. 1, pp. 128-133. 
Hommes, C.H. (2005), "Heterogeneous Agent Models in Economics and Finance", Tinbergen Institute Discussion Paper, No. 05-056/1, Amsterdam and Rotterdam.

Honderich, T. (2005), The Oxford Companion to Philosophy, 2nd edition online, Oxford University Press, available at www.oxfordreference.com (accessed on 18 September 2015).

Hull, J.C. (1997), Options, Futures, and Other Derivates, 7th edition 2009, Pearson Prentice Hall, N.J.

Jensen, M.C. (1968), "The Performance of Mutual Funds in the Period 19451964”, Journal of Finance, Vol. 23 No. 2, pp. 389-416.

Jensen, M.C. \& Meckling, W.H. (1976), "Theory of the Firm: Managerial Behavior, Agency Costs and Ownership Structure", Journal of Financial Economics, Vol. 3 No. 4, pp. 305-360.

Johnson, S.B. (2001), Emergence: The Connected Lives of Ants, Brains, Cities, and Software, Alan Lane, The Pinguin Press, London.

Jovanovic, F. (2006a), "Economic instruments and theory in the construction of Henri Lefèvre's 'science of the stock market'”, in Poitras, G. (ed.) (2006), Pioneers of Financial Economics, Volume 1: Contributions Prior to Irving Fisher, Edward Elgar, Cheltenham.

Jovanovic, F. (2006b), “A nineteenth-century random walk: Jules Regnault and the origines of scientific financial economics", in Poitras, G. (ed.) (2006), Pioneers of Financial Economics, Volume 1: Contributions Prior to Irving Fisher, Edward Elgar, Cheltenham.

Jovanovic, F. (2008), “The Construction of the Canonical History of Financial Economics", History of Political Economy, Vol. 40 No. 2, pp. 213-242. 
Kahneman, D. (2003), "A Perspective on Judgment and Choice, Mapping Bounded Rationality", American Psychologist, Vol. 58 No. 9, pp. 697-720.

Kahneman, D. (2011), Thinking, Fast and Slow, Penguin book edition 2012, Penguin Books, London.

Kahneman, D. \& Frederick, S. (2002), "Representativeness revisited: Attribute substitution in intuitive judgment”, pp. 49-81 in Gilovich, T., Griffin, D., Kahneman, D. (eds.), Heuristics \& Biases: The Psychology of Intuitive Judgment, Cambridge University Press, New York.

Karr, A.F. (2008), "Martingale”, in Durlauf, S.N \& Blume. L.E. (eds.) (2008), The New Palgrave Dictionary of Economics, 2nd edition, available at http://www.dictionaryofeconomics.com/ (accessed on 10 november 2014).

Kendall, M.G. (1953), “The Analysis of Economic Time-Series, Part I: Prices”, Journal of the Royal Statistical Society, Series A (General), Vol. 116 No. 1, pp. 11-25.

Keynes, J.M. (1921), A Treatise on Probability, edition 2008 from Rough Draft Printing, Originally published by MacMillan and Co, London.

Keynes, J.M. (1936), The General Theory of Employment, Interest, and Money, edition 1997 from Prometheus Books, New York, originally published by Harcourt, Brace \& World, New York.

Keynes, J.M. (1937), The Collected Writings of John Maynard Keynes, vol. XIV, edited by Johnson, E. \& Moggridge, D., 1978, Cambridge University Press, Cambridge, originally published as "The General Theory of Employment" in Quarterly Journal of Economics, February 1937, pp. 109123.

Keynes, J.M. (1939), “Professor Tinbergen's Method”, Economic Journal, Vol. 49 No. 195 , pp. 558-577. 
Kindleberger, C. (1978), Manias, Panics, and Crashes: A History of Financial Crises, 4th edition 2000, Wiley, New York.

Kirman, A. in Durlauf, S.N \& Blume, L.E. (eds.) (2008), The New Palgrave Dictionary of Economics, 2nd edition, available at http://www.dictionaryofeconomics.com/ (accessed on 1 november 2014).

Kirzner, I.M. (1985), Discovery and the Capitalist Process, The University of Chicago Press, Chicago.

Klamer, A. (2001), "Making sense of economists: from falsification to rhetoric and beyond”, Journal of Economic Methodology, Vol. 8 No. 1, pp. 69-75.

Klamer, A. (2003), “A pragmatic view on values in economics", Journal of Economic Methodology, Vol. 10 No. 2, pp. 191-212.

Klamer, A. (ed.) (2006), The Value of Culture: On the Relationships between Economics and Art, Amsterdam University Press, Amsterdam.

Klamer, A. (2007), Speaking of Economics: How to Get in the Conversation, Routledge, London and New York.

Klamer, A. (2009), Rethoric, in Peil, J.J.M. \& Van Staveren, I.P. (eds.), Handbook of economics and ethics, Edward Elgar, Cheltenham, pp. 449-454

Klamer, A. (2014), Doing the right thing, draft of January 2014.

Klamer, A. \& Leonard T.C. (1994), So What's an Economic Metaphor?, pp. 20-51 in Mirowski, P. (ed.), Natural Images in Economics: Markets Read in Tooth and Claw, Cambridge University Press, Cambridge.

Klamer, A. \& McCloskey, D.N. (1988), Economics in the human conversation, in The consequences of economic rhetoric, Klamer, A., McCloskey, D.N. \& 
Solow, R.M. (eds.), Cambridge University Press, Cambridge, New York, pp. 3-20.

Klamer, A. \& McCloskey, D.N. (1992), "Accounting as the master metaphor of economics", European Accounting Review, 1992, Vol. 1 No. 1, pp. 145160.

Klamer, A., McCloskey, D.N. \& Solow, R.M. (eds.) (1988), The consequences of economic rhetoric, Cambridge University Press, Cambridge, New York.

Knight, F.H. (1921), Risk, Uncertainty and Profit, New edition from Signalman Publishing, Orlando, 2009, originally published by Hart, Schaffner and Marx, Houghtin Mifflin Co.

Kocken, T.P. (1997), Financial Risk Management: Theorie en praktijk voor financiële en niet-financiële Instellingen, 2nd edition 2010, Tutein Nolthenius, Den Bosch.

Kocken, T.P. (2006), Curious Contracts: Pension Fund Redesign for the Future, Tutein Nolthenius, Den Bosch.

Kocken, T.P. (2012), Inaugural Lecture: “Endogenous Instability: You Don’t Want to Think about It!", Cardano, Rotterdam.

Koedijk, K., \& Slager, A.M.H. (2011), Beleggen met visie: Handboek voor beleggers, bestuurders en beslissers, Uitgeverij Balans, Amsterdam.

Kuhn, T.S. (1962), The Structure of Scientific Revolutions, 3rd edition 1996, University of Chicago Press, Chicago.

Langlois, R.N. (1982), Subjective Probability and Subjective Economics, New York University, New York. 
Langlois, R.N. \& Cosgel, M.M. (1993), "Frank Knight on Risk, Uncertainty, and the Firm: a New Interpretation", Economic Inquiry, Vol. 31 No. 3, pp. 456465 .

Lanham, R.A. (1991), A Handlist of Rhetorical Terms, 2nd edition, University of California Press, Berkeley.

Law, J. (ed.) (2014), A Dictionary of Finance and Banking, 5th edition online, Oxford University Press, Oxford, available at http://www.oxfordreference.com/(accessed on 14 September 2014).

Lentricchia, F., \& McLaughlin, T. (1995), Critical Terms for Literary Study, 2nd edition, University of Chicago Press, London.

LeRoy, S.F. \& Singell Jr, L.D (1987), "Knight on Risk and Uncertainty”, Journal of Political Economy, Vol. 95 No. 2, pp. 394-406.

Lewis, M. (1989), Liar's Poker, Hodder and Stoughton, London.

Lewis, M. (2010), The Big Short: Inside the Doomsday Machine, W.W. Norton, New York.

Lintner, J. (1965), "The Valuation of Risk Assets and the Selection of Risky Investments in Stock Portfolios and Capital Budgets", The Review of Economics and Statistics, Vol. 47 No. 1, pp. 13-37.

Lucas, R.E. Jr. (1978), “Asset Prices in an Exchange Economy”, Econometrica, Vol. 46 No. 6, pp. 1429-1446.

Lucas, R.E. \& Sargent, T.J. (1981), Rational Expectations and Econometric Practices, University of Minnesota Press, Minneapolis.

MacKenzie, D.A. (2006), An Engine, Not a Camera: How Financial Models Shape Markets, paperback edition 2008, The MIT Press, Cambridge, MA. 
Macaulay, F.R. (1938), Some Theoretical Problems Suggested by the Movements of Interest Rates, Bond Yields and Stock Prices in the United States since 1856, NBER, available at http://admin.nber.org/authors/frederick_macaulay (accessed on 11 December 2014).

Magala, S. (2004), "Cross-cultural compromises, multiculturalism and the actuality of unzipped Hofstede”, ERIM Report Series Research in Management, Rotterdam School of Management, Rotterdam.

Magala, S. (2005), Cross-Cultural Competence, Routledge, London and New York.

Maginn, J.L. \& Tuttle, D.L. (eds.) (1983), Managing Investment Portfolios: A Dynamic Process, 2nd edition 1990, Warren, Gorham \& Lamont, Boston.

Mandelbrot, B.B. (1963a), "The Variation of Certain Speculative Prices”, The Journal of Business, Vol. 36, No. 4, pp. 394-419.

Mandelbrot, B.B. (1963b), "New Methods in Statistical Economics", Journal of Political Economy, Vol. 71 No. 5, pp. 421-440.

Mandelbrot, B.B. \& Hudson, R.L. (2004), The (Mis)Behaviour of Markets: A Fractal View of Risk, Ruin and Reward, paperback edition 2008, Profile Books, London, originally published by Basic Books, United States.

Markowitz, H.M. (1952), "Portfolio Selection", The Journal of Finance, Vol. 7 No. 1, pp. 77-91.

Markowitz, H.M. (1959), Portfolio Selection: Efficient Diversification of Investments, 2nd edition, 1991, Blackwell Publishing, Malden, originally published by John Wiley \& Sons. 
Markowitz, H.M. (1990), "Harry M. Markowitz - Prize Lecture: Foundations of Portfolio Theory", available at http://www.nobelprize.org/nobel_prizes/economics/laureates/ 1990/markowitz-lecture.html (accessed on 24 February 2013).

Markowitz, H.M. (1999), "The Early History of Portfolio Theory: 1600-1960", Financial Analysts Journal, Vol. 55 No. 4, pp. 5-16.

Marshall, A. (1890), Principles of Economics, 8th edition, 1920, MacMillan \& Co, London.

McCloskey, D.N. (1983), "The Rhetoric of Economics", Journal of Economic Literature, Vol. 21 No. 2, pp. 481-517.

McCloskey, D.N. (1985a), The Rhetoric of Economics, 1st edition, University of Wisconsin Press, Wisconsin.

McCloskey, D.N. (1985b), The Applied Theory of Price, 2nd edition, Macmillan Publishing Company, New York.

McCloskey, D.N. (1985c), "Sartorial Epistemology in Tatters: A Reply to Martin Hollis", Economics and Philosophy, Vol. 1 No. 1, pp. 134-137.

McCloskey, D.N. (1990), If You're So Smart: The Narrative of Economic Expertise, University of Chicago Press, Chicago.

McCloskey, D.N. (1994), Knowledge and Persuasion in Economics, Cambridge University Press, Cambridge.

McCloskey, D.N. (1996a), The Vices of Economists; The Virtues of the Bourgeoisie, University of Amsterdam Press, Amsterdam. 
McCloskey, D.N. (1996b), “Missing Ethics in Economics”, pp. 187-201 in Klamer, A. (ed.) (2006), The Value of Culture: On the Relationships Between Economics and Arts, Amsterdam University Press, Amsterdam.

McCloskey, D.N. (1998), The Rhetoric of Economics, 2nd edition, University of Wisconsin Press, Wisconsin.

McCloskey, D.N. (2000), How to Be Human-Though an Economist, University of Michigan Press, Ann Arbor.

McCloskey, D.N. (2006), The Bourgeois Virtues, Ethics for an Age of Commerce, The University of Chicago Press, Chicago.

McCloskey, D.N. (2010), Bourgeois Dignity: Why Economics Can't Explain the Modern World, The University of Chicago Press, Chicago.

McCloskey, D.N. \& Klamer A. (1995), “One Quarter of GDP is Persuasion”, The American Economic Review, Vol. 85 No. 2, pp. 191-195.

McSweeney, B. (2002), “Hofstede's model of national cultural differences and their consequences: A triumph of faith - a failure of analysis", Human Relations, Vol. 55 No. 1, pp. 89-118.

Merton, R.C. (1973a), "An Intertemporal Capital Asset Pricing Model", Econometrica, Vol. 41 No. 5, pp. 867-887.

Merton, R.C. (1973b), “Theory of Rational Option Pricing”, The Bell Journal of Economics and Management Science, Vol. 4 No. 1, pp. 141-183.

Merton, R.K. (1948), "The Self-Fulfilling Prophecy”, The Antioch Review, Vol. 8 No. 2, pp. 193-210.

Minsky, H.P. (1982), "The Financial-Instability Hypothesis: Capitalist Processes and the Behavior of the Economy", pp. 13-39 in Kindleberger, C.P. \& 
Laffargue, JP. (eds.), Financial Crises: Theory, History, and Policy, Cambridge University Press, Cambridge, paper 282 of the Hyman P. Minsky Archive available at http://digitalcommons.bard.edu/ hm_archive/282 (accessed on 1 February 2015).

Mirowski, P. (1989), More Heat than Light: Economics as Social Physics, Physics as Nature's Economics, paperback edition 1999, Cambridge University Press, Cambridge.

Mirowski, P. (2013), Never Let a Serious Crisis Go to Waste: How Neoliberalism survived the Financial Meltdown, paperback edition 2014, Verso, London.

Mirowski, P. \& Sent, E.M. (2002), Science Bought and Sold: Essays in the Economics of Science, The University of Chicago Press, Chicago.

Morgan, G. (1986), Images of Organization, updated edition 2006, SAGE Publications, Thousand Oakes, London, New Delhi.

Mossin, J. (1966), "Equilibrium in a Capital Asset Market”, Econometrica, Vol. 34 No. 4, pp. 768-783.

Muth, J.F. (1961), "Rational Expectations and the Theory of Price Movements", Econometrica, Vol. 29 No. 3, pp. 315-335.

Newbold, P. (1984), Statistics for Business and Economics, Prentice Hall, N.J.

Perelman, C., \& Olbrechts-Tyteca, L. (1958), The New Rhetoric: A Treatise on Argumentation, translated by Wilkinson, J. \& Weaver, P., 1969, University of Notre Dame Press, Notre Dame.

Perelman, C. (1979), The New Rhetoric and the Humanities: Essays on Rhetoric and its Applications, translated by Kluback, W., D. Reidel Publishing Company, Dordrecht, Holland. 
Petram, L. (2011), De bakermat van de beurs: Hoe in zeventiende eeuws Amsterdam de moderne aandelenhandel ontstond, Uitgeverij Atlas, Amsterdam en Antwerpen.

Piketty, T. (2014), Capital in the Twenty-First Century, The Belknap Press of Harvard University Press, Cambridge, MA.

Pistorius, T. (1991), Verschillende risicomaatstaven in het licht van de efficiënte set van portefeuilles van objecten, MSc.-thesis in Finance, University of Tilburg, Tilburg, Netherlands.

Pistorius, T. (2004), Asset mix en horizon, research paper of restricted distribution, IRIS/Robeco Research, Rotterdam.

Pistorius, T. (2014), "Beyond Statistics: A New Rhetoric for Investment Theory", Journal of Organizational Change Management, Vol. 25 No. 5, pp. 722731.

Plato (1914), Euthyphro, Apology, Crito, Phaedo, Phaedrus, translated by Fowler, H.N., Harvard University Press Cambridge, MA.

Plato (1925), Lysis, Symposium, Gorgias, translated by Lamb, W.R.M., Harvard University Press Cambridge, MA.

Poitras, G. (1996), "From Commercial Arithmetic to Life Annuities: The Early History of Financial Economics, 1478-1776.”, Simon Fraser University and National University of Signapore, available at http://beedie.sfu.ca/homes/poitras/FIN_HIS3.pdf (accessed on 14 September 2014).

Poitras, G. (ed.) (2006), Pioneers of Financial Economics, Volume 1: Contributions Prior to Irving Fisher, Edward Elgar, Cheltenham. 
Poitras, G. (ed.) (2007), Pioneers of Financial Economics, Volume 2: TwentiethCentury Contributions, Edward Elgar, Cheltenham.

Polanyi, M. (1958), Personal Knowledge: Towards a Post-Critical Philosophy, corrected edition 1962, paperback edition 1974, University of Chicago Press, Chicago.

Popescu, A.V. (2015), “Complexity”, Cardano Group Research, draft version.

Preda, A. (2006), Rational investors, informative prices: the emergence of the 'science of financial investments' and the random walk hypothesis, in Poitras, G. (ed.) (2006), Pioneers of Financial Economics, Volume 1: Contributions Prior to Irving Fisher, Edward Elgar, Cheltenham.

Quintilian, M.F. (1920), Institutio Oratoria (The Orators Education), translated by Butler, H.E., Harvard University Press Cambridge, MA.

Read, C. (2013), The Efficient Market Hypothesists: Bachelier, Samuelson, Fama, Ross and Shiller, Palgrave Macmillan, Houndmills, Basingstoke, Hampshire.

Regnault, J.A.F. (1863), Calcul des chances et philosophie de la Bourse, MalletBachelier et Castel, Paris, available at https://ia600506.us.archive.org/ 12/items/ calculdeschances00regn/calculdeschances00regn.pdf (accessed on 30 November 2014).

Reilly, F.K. (1994), Investment Analysis and Portfolio Management, 4th edition, Dryden Press, Fort Worth.

Reiss, J. (2013), Philosophy of Economics: A Contemporary Introduction, Routledge, New York.

Roberts, H.V. (1959), "Stock-Market 'Patterns' and Financial Analysis: Methodological Suggestions", Journal of Finance, Vol. 14 No 1, pp. 1-10. 
Rorty, R.M. (1979), Philosophy and the Mirror of Nature, Princeton University Press, Princeton.

Rosenberg, K. \& Kocken T.P. (2013), "Behavioural biases and not-so-optimal portfolio construction", Professional Investor (Journal of CFA Society United Kingdom), atumn 2013, pp. 29-32.

Ross, S.A. (1976), "The Arbitrage Theory of Capital Asset Pricing", Journal of Economic Theory, Vol. 13 No. 3, pp. 341-360.

Ross, S.A., Westerfield, R.W. \& Jaffe, J. (1988), Corporate Finance, 4th edition, 1996, IRWIN, Chicago.

Rothbard, M.N. (1991), "The End of Socialism and the Calculation Debate Revisited", Review of Austrian Economics, Vol. 5 No. 2, pp. 51-76.

Roy, A.D. (1952), "Safety First and the Holding of Assets", Econometrica, Vol. 20 No. 3, pp. 431-449.

Roy, A.D. (1961), "Portfolio Selection by Harry Markowitz", Econometrica, Vol. 29 No. 1, pp. 99-100.

Rubinstein, M.E. (2006a), A History of the Theory of Investments: My Annotated Bibliography, John Wiley \& Sons , N.J.

Rubinstein, M. (2006b), "Bruno de Finetti and Mean-Variance Portfolio Selection", Journal of Investment Management, Vol. 4, No. 3

Samuelson, P.A. (1965a), "Rational theory of warrant pricing", Industrial Management Review, Vol. 6 No. 2, pp. 13-39.

Samuelson, P.A. (1965b), "Proof that properly anticipated prices fluctuate randomly", Industrial Management Review, Vol. 6 No. 2, pp. 41-49. 
Samuelson, P.A. (1969a), "Lifetime Portfolio Selection by Dynamic Stochastic Programming", Review of Economics and Statistics, Vol. 51 No. 3, pp. 239-246.

Samuelson, P.A. (1969b), Classical and Neoclassical Theory, In Clower, R.W., ed., Monetary Theory, Penguin, London.

Samuelson, P.A. (1974), "Challenge to Judgment", The Journal of Portfolio Management, Vol. 1 No. 3, pp. 17-19.

Sargent, T.J. (2008), "Rational Expectations", The Concise Encyclopedia of Economics, available at http://www.econlib.org/library/Enc/Rational Expectations.html (accessed on 20 September 2013).

Savage, L.J. (1954), The Foundations of Statistics, John Wiley and Sons, New York.

Schelling, T.C. (1978), Micromotives and Macrobehavior, $2^{\text {nd }}$ edition 2006, W.W. Norton \& Company, New York.

Schilp, M. (ed.) (2015), "Economen leven in een fantasiewereld", translated as "Economists live in a fantasy world", Ad Valvas edition 28 January 2015, VU University Amsterdam, pp. 18-22, accessed on 9 May 2015 at http://issuu.com/advalvas/docs/nr._11_28_januari_2015

Schön, D.A. (1970), "Reith Lectures 1970, Change and Industrial Society Lecture 1: The Loss of the Stable State", transmitted on 15 November 1970, BBC Radio 4, available at http://downloads.bbc.co.uk/rmhttp/radio4/ transcripts/ 1970_reith1.pdf (accessed on 9 May 2015).

Schumpeter, J.A. (1911), The Theory of Economic Development: An Inquiry Into Profits, Capital, Credit, Interest, and the Business Cycle, translated 1934, edition 1951, Harvard University Press, Cambridge MA. 
Shackle, G.L.S. (1955), Uncertainty in Economics and Other Reflections, paperback edition 2010, Cambridge University Press, Cambridge.

Sharpe, W.F. (1963), “A Simplified Model for Portfolio Analysis”, Management Science, Vol. 9 No. 2, pp. 277-293.

Sharpe, W.F. (1964), "Capital Asset Prices: A Theory of Market Equilibrium under Conditions of Risk", The Journal of Finance, Vol. 19 No 3, pp. 425-442.

Shefrin, H. (1999), Beyond Greed and Fear: Understanding Behavioral Finance and the Psychology of Investing, revised version 2002, Oxford University Press, New York.

Shell (2008), "Scenarios: An Explorers Guide", available at http://www.shell.com/global/future-energy/scenarios/explorers-guide.html (accessed on16 May 2015).

Shell (2014), "New Lens Scenarios", available at http://www.shell.com/global/future-energy/scenarios/new-lensscenarios.html (accessed on 23 September 2015).

Shiller, R.J. (1981), "Do Stock Prices Move Too Much to Be Justified by Subsequent Changes in Dividends?, The American Economic Review, Vol. 71 No. 3, pp. 421-436.

Shiller, R.J. (2000), Irrational Exuberance, Princeton University Press, paperback edition of 2001, Broadway Books.

Shiller, R.J. \& Akerlof, G. (2009), Animal Spirits: How Human Psychology Drives the Economy And Why It Matters for Global Capitalism, Princeton University Press, Princeton, and Oxford.

Shleifer, A. \& Vishny, R.W. (1997), "The Limits of Arbitrage", The Journal of Finance, Vol. 52 No. 1, pp. 35-55. 
Siegel, J.J. (1994), Stocks for the Long Run: the Definitive Guide to Financial Market Returns and Long-Term Investment Strategies, 3rd edition 2002, McGraw-Hill, New York.

Simon, H.A. (1955), "A Behavioral Model of Rational Choice", Quarterly Journal of Economics, Vol. 69 No. 1, pp. 99-118.

Skidelsky, R.J.A. (2009a), Keynes: The Return of the Master, PublicAffairs, New York.

Skidelsky, R.J.A. (2009b), "Economic theory: How to rebuild a newly shamed subject”, Financial Times, edition of 13 December 2009.

Slager, A.M.H. \& Koedijk, K. (2007), "Investment beliefs: Every Asset Manager Should Have Them", Journal of Portfolio Management, Vol. 33, No. 3, pp. $77-84$.

Smith, A. (1790), The Theory of Moral Sentiments, edition 2009, Penguin Books Ltd, London.

Solnik, B. (1996), International Investments, 3rd edition, Addison-Wesley Publishing Company, Reading, Massachusetts.

Soros, G. (1987), The Alchemy of Finance, edition 1994, John Wiley \& Sons, New York.

Soros, G. (2008), The New Paradigm for Financial Markets: The Credit Crisis of 2008 and What It Means, PublicAffairs, New York.

Stichting Shell Pensioenfonds (2015), Jaarverslag 2014, available at http://www.shell.nl/pensioenfonds/nl/about-pensionfund/informatie-ennieuws/documenten-en-informatie/annual-reports.html (accessed on 16 May 2015). 
Sullivan E.J. (2011), “A.D. Roy: The Forgotten Father of Portfolio Theory”, in Biddle, J.E. \& Emmett R.B. (eds.), Research in the History of Economic Thought and Methodology, Vol. 29 Part 1, Emerald Group Publishing, pp. 73-82.

Sylla, E.D. (2006), Commercial arithmic, theology, and the intellectual foundation of Jacob Bernouilli's Art of Conjecturing, in Poitras, G. (ed.) (2006), Pioneers of Financial Economics, Volume 1: Contributions Prior to Irving Fisher, Edward Elgar, Cheltenham.

Taleb, N.N. (2007), The Black Swan: The Impact of the Highly Improbable, 2nd edition, 2010, Random House, New York.

Taleb, N.N. (2010), "Why did the Crisis of 2008 Happen?”, draft 3rd version August 2010, available at http://ssrn.com/abstract=1666042 (accessed at 11 May 2014).

Tett, G. (2009), Fool's Gold: How the Bold Dream of a Small Tribe at J.P. Morgan Was Corrupted by Wall Street Greed and Unleashed a Catastrophe, Free Press, New York.

Thaler, R.H. \& Sunstein, C.R. (2008), Nudge: Improving Decisions About Health, Wealth and Happiness, Penguin book edition 2009, Penguin Books, London.

Tobin, J. (1958), "Liquidity Preference as Behavior Towards Risk", The Review of Economic Studies, Vol. 25, No. 2, pp. 65-86.

Tobin, J. (2008), "Fisher, Irving (1867-1947).", The New Palgrave Dictionary of Economics, in Durlauf, S.N \& Blume, L.E. (eds.) (2008), The New Palgrave Dictionary of Economics, 2nd edition, available at http://www.dictionaryofeconomics.com/(accessed on 25 February 2015). 
Treynor, Jack L. (1962), "Toward a Theory of Market Value of Risky Assets", unpublished manuscript.

Toulmin, S.E. (1958), The Uses of Argument, Cambridge University Press, Cambridge.

Tversky, A. \& Kahneman, D. (1974), "Jugdment under Ucertainty: Heuristics and Biases", Science, Vol. 185 No. 4157, pp. 1124-1131.

Tversky, A. \& Kahneman, D. (1979), " Prospect Theory: An Analysis of Decision under Risk", Econometrica, Vol. 47 No. 2, pp. 263-291.

Van Capelleveen, H.F., Kat, H.M. \& Kocken, T.P. (2004), "How Derivatives Can Help Solve the Pension Fund Crisis: An Ironic Result", Journal of Portfolio Management, Vol. 30 No. 4, pp. 244-253.

Van Dillen, J.G. (1935), Isaac Le Maire and the Share Trading of the Dutch East India Company, original title "Isaac le Maire et le commerce des actions de la Compaigne de Indes Orientales", Revue d'Histoire Moderne, Vol 5. No. 21, pp. 121-137, translated by Majithia, A. in Poitras, G. (ed.) (2006), Pioneers of Financial Economics, Volume 1: Contributions Prior to Irving Fisher, Edward Elgar, Cheltenham.

Van Dillen, J.G., Poitras, G. \& Majithia, A. (2006), Isaac Le Maire and the Share Trading of the Dutch East India Company, in Poitras, G. (ed.) (2006), Pioneers of Financial Economics, Volume 1: Contributions Prior to Irving Fisher, Edward Elgar, Cheltenham.

Van de Mieroop, M. (2005), The Invention of Interest: Sumerian Loans, in Goetzmann, W.N. \& Rouwenhorst K.G. (eds.) (2005), The Origins of Value: The Financial Innovations That Created Modern Capital Markets, Oxford University Press, Oxford. 
Velleman, P.F. (2008), “Truth, Damn Truth, and Statistics”, Journal of Statistics Education, Vol.16 No. 2, pp. 1-14.

Vereniging voor Beleggingsanalisten, VBA, number of members, available at http://www.vbabeleggingsprofessionals.nl/over-de-vba/leden (accessed on 7 April 2015).

Von Mises, L. (1949), Human Action: A Treatise on Economics, edition 2012 from Martino Publishing, Mansfield Centre, originally published by Yale University Press, New Haven.

Von Mises, L. (1962), The Ultimate Foundation of Economic Science, An Essay On Method, D. van Nostrand Company, Princeton, N.J.

Vickers, B. (1988), In Defence of Rhetoric, edition 1997, Oxford University Press, Oxford.

Walschots, B. \& Capelleveen, H. (2008), "Hybride bescherming; de case van het Rabobank Pensioenfonds" ("Hybrid Protection, the case of the Pension Fund of the Rabobank", my translation), VBA Journaal, Vol. 24 No. 2, pp. $40-47$.

Weick, K.E. (2001), Making Sense of the Organization, Blackwell Publishers, Oxford.

Wikipedia, "Roulette", available at http://en.wikipedia.org/wiki/Roulette (accessed on 6 October 2013).

Wilde, O.F.O.W. (1891), The Picture of Dorian Gray, available at http://www.gutenberg.org/files/174/174-h/174-h.htm (accessed on 9 May 2015).

Wilkinson, A. \& Kupers, R. (2013), "Living in the Futures", Harvard Business Review, Vol. 91 No. 5, pp. 119-127. 
Williams, J.B. (1938), The Theory of Investment Value, Harvard University Press, Cambridge MA.

Working, H. (1934), “A Random-Difference Series for Use in the Analysis of Time Series", Journal of the American Statistical Association, Vol. 29 No. 185, pp. 11-24.

Worthington, I. (ed.) (2007), A Companion to Greek Rhetoric, paperback edition 2010, Wiley-Blackwell, West Sussex.

Wubben, E.F.M. (1993), Markets, Uncertainty and Decision-making, A History of the Introduction of Uncertainty into Economics, Thesis Publishers, Amsterdam.

Ziliak, S.T. \& McCloskey, D.N. (2008), The Cult of Statistical Significance: how the Standard Error Costs us Jobs, Justice, and Lives, The University of Michigan Press, Ann Harbor.

Zimmermann H. \& Hafner, W. (2006), Vinzenz Bronzin's option pricing theory: contents, contribution and background, in Poitras, G. (ed.) (2006), Pioneers of Financial Economics, Volume 1: Contributions Prior to Irving Fisher, Edward Elgar, Cheltenham. 


\section{Acknowledgements}

For me the writing of a dissertation was a natural part of my Bildung, so I start with thanking my parents who have encouraged me long ago to engage in intellectual projects like this. Nevertheless, my first thanks go to my wife Mieke, I could not have written the dissertation without her support.

Next, I would like to thank the support of the staff of the PID-program (Promoveren in Deeltijd), that is the former part-time PhD-program at the Rotterdam School of Management at the Erasmus University. Frits van Engeldorp Gastelaars was ready to help and willing to share his knowledge of science and the writing of dissertations. Marja Flory coached me and shared her international academic network with me. I have had for example a number of talks with Deirdre McCloskey, a main critic of mainstream economics.

It has been a pleasure to work with my promotor, Slawek Magala. His intellectual strength combined with his hands-on management of the process have been of benefit to the dissertation. The members of the promotion committee have had distinctive influence on the definitive form of the dissertation: Arjo Klamer introduced me to the culture of economics, Abe de Jong suggested to investigate the history of finance and innovative cases in practice, and Theo Kocken has stimulated my interest in evolutionary finance and many other topics of heterodox finance and practice.

I also would like to thank the many others who contributed in my thought process: my fellow students and other staff in the PID-program, the interviewees, the academics I met to talk about my dissertation, the colleagues in the investment community, friends, family, and all the other people who have shown interest in my project. 


\section{Summary}

Uncertainty is a feeling of anxiety and a part of culture since the dawn of civilization. Civilizations have invented numerous ways to cope with uncertainty. Technology has been directed against natural uncertainties, law against behavioural uncertainties, and religion against transcendental uncertainties. In investment theory, the technology of mathematical statistics treats uncertainty of the future outcomes of financial markets by the metaphor of the machine, as if investments can be engineered to a maximum efficiency of risk and return, and financial markets process information in a machine-like efficient way. Yet, the competing economic theory of uncertainty stresses for example the metaphor of the organism, which highlights change instead of stability.

The rhetoric as the discourse of investment theory uncovers that the theory of statistics is a blind spot in the current conversation about investment theory and practice. Probability and prediction in investment theory look like a tying sale, since investment theory is founded on stochastical predictability. The proof of unpredictability lies outside the paradigm of statistics, because statistics assumes that the substrate, that produces probability outcomes, is stable. The inference to the best explanation that investment returns are unpredictable, seems more persuasive than the arguments of investment theory that personal or objective probability beliefs predict.

The theory of objective probabilities founds the Capital Asset Pricing Model (CAPM) but does not stochastically predict, and neither do Markowitz's subjective probabilities for his portfolio theory. The ergodic assumption as used in the rational expectation hypothesis assumes that objective probability information is available from past data. The assumption which accommodates stochastical 
changes, is needed to make financial economics a predictive science. But past data cannot predict the future stochastically because unexpected changes occur.

For example Keynes's theory of uncertain expectations contrasts the contemporary investment theory of the CAPM, and seems more relevant. His alternative investment theory implies that rational expectations do not lead to predictability, but to instability. To my opinion the efficient market hypothesis did not go far enough be merely denying predictability in active management, but should have led to the logical conclusion that return and risk of financial markets are unpredictable either.

Statistical models for investing have a function, despite that they cannot predict: they reveal how stakeholders of a pension fund or parties in an option contract divide risk and return: in essence, the models try to quantify statistically the equity, the fairness of the distribution of risk and return between parties involved. The quantification serves to make the fairness between stakeholders or parties visible, and is not meant, or should be understood, as a specific prediction. The statistical model is useful, because it provides an insight which would otherwise not have been available.

In the dissertation, a lot of ideas to change finance have had attention: the alternative statistical theory of Mandelbrot, bubble theory, political finance, the analysis of rhetoric, the analysis of culture, innovative practices, ethics in the form of virtue and value ethics, and the history of finance. A more heterogeneous culture of academic finance and investment practice could accommodate the ideas mentioned: the alternatives for modern investment theory are plenty. How to make the culture of finance more heterogeneous? That starts with awareness of the current problems, the introduction of multiform metaphors for investment theory, and handling the ambiguity of theory uncertainty, by presenting explaining and elaborating the alternatives. 


\section{Samenvatting}

Onzekerheid is een gevoel van ongerichte angst en een onderdeel van cultuur sinds het begin van de beschaving. Beschavingen hebben tal van manieren bedacht om onzekerheid te bestrijden. Zo is technologie gericht tegen onzekerheden uit de natuur, zijn wetten ontwikkeld tegen onzekerheden in het gedrag van mensen, en is religie ontstaan als antwoord op transcendentale onzekerheden. In de beleggingstheorie, behandelt wiskundige statistiek onzekerheid over de toekomstige rendementen op de financiële markten met behulp van de machine metafoor, alsof het risico en rendement van beleggingen tot een efficiënt niveau kunnen worden geconstrueerd, en financiële markten informatie machinaal op een efficiënte manier verwerken. De concurrerende economische theorie die onzekerheid juist centraal stelt, gaat bijvoorbeeld uit van de metafoor van het organisme, die verandering in plaats van stabiliteit benadrukt.

De theorie van de statistiek vormt een blinde vlek in de huidige conversatie over de beleggingstheorie en praktijk. Waarschijnlijkheidstheorie en voorspelbaarheid van beleggingsrendementen lijken wel een koppelverkoop, omdat beleggingstheorie is gebaseerd op stochastische voorspelbaarheid. Het bewijs van onvoorspelbaarheid ligt echter buiten het paradigma van de statistiek, want de statistiek veronderstelt dat de ondergrond die uitkomsten produceert, stabiel is. De meest plausibele verklaring lijkt echter dat beleggingsrendementen onvoorspelbaar zijn. De argumenten voor onvoorspelbaarheid lijken overtuigender dan de argumenten van de beleggingstheorie voor voorspelbaarheid, die mede zijn gebaseerd op de statistische theorieën.

De statistische theorie van objectieve waarschijnlijkheid fundeert het marktevenwichtsmodel op van de financiële markten, het Capital Asset Pricing Model (CAPM). De ergodische veronderstelling, zoals gebruikt in de hypothese van rationele verwachtingen gaat ervan uit dat objectieve waarschijnlijkheden 
afleidbaar zijn uit historische gegevens. Deze veronderstelling is nodig om van financiële economie een voorspellende wetenschap te maken. Maar gegevens uit het verleden voorspellen niet op een stochastische manier, omdat in de toekomst onverwachte veranderingen optreden.

Keynes' theorie over onzekere verwachtingen staat in contrast tot het CAPM en lijkt relevanter. Zijn alternatieve beleggingstheorie impliceert dat rationele verwachtingen niet leiden tot voorspelbaarheid, maar tot instabiliteit. Naar mijn mening is de efficiënte markt hypothese in het CAPM niet ver genoeg doorgetrokken: wel ontkent het CAPM de mogelijkheid van extra rendement door actief beheer op de financiële markten, maar het CAPM gaat desalniettemin uit van de voorspelbaarheid van risico en rendement van financiële markten.

Statistische modellen voor beleggen hebben als functie dat ze in kaart brengen hoe belanghebbenden bij bijvoorbeeld een pensioenfonds, of partijen in een optiecontract, risico en rendement verdelen: in essentie proberen dit type modellen met statistiek de rechtvaardigheid van de verdeling van risico en rendement tussen de betrokken partijen te kwantificeren. Deze kwantificering is echter geen voorspelling.

In het proefschrift hebben diverse ideeën om de beleggingstheorie en de praktijk te veranderen aandacht gekregen: de alternatieve statistische theorie van Mandelbrot, zeepbeltheorie, politieke finance, de analyse van de retoriek, de analyse van de cultuur, innovatieve praktijken, ethiek in de vorm van deugd- en waarde-ethiek, en de geschiedenis van finance. De alternatieve beleggingstheorieën verdienen meer aandacht in de universitaire wereld. En, hoe kan cultuur van beleggers de alternatieve beleggingstheorieën adopteren? Dat begint met een groter bewustzijn van de problemen van de huidige beleggingstheorie, de omarming van een pluriforme benadering van beleggingstheorie, en het verder uitwerken van de alternatieve beleggingstheorieën en deugd- en waarde ethiek, in theorie en praktijk. 


\section{About the Author}

Thomas Pistorius was born in 1965 in Dongen in the Netherlands. In 1991 he obtained a MSc in finance (in Dutch: drs.) from Tilburg University. In 1999 he finished the postgraduate VBA-education, the Dutch CFA-equivalent for becoming an investment and financial analyst. In his professional life he has for the last 20 years been working in investment management as an investment advisor, analyst, risk manager, and researcher.

His research interest as a MSc-student in finance and as a researcher at the investment management company Robeco at the research institute IRIS has been on mathematical-statistical analysis of investment theory. Besides his interest in the science of finance, he is also attracted to arts. In September 2010, he completed a BA in philosophy at Utrecht University. In his dissertation finance and arts meet in the form of the rhetoric, history, philosophy, and culture of investment theory to discuss the problem of stochastical predictability.

\section{Publications}

- De schatkamers van de beleggingstheorie van voor de jaren 50. 2015. VBAjournaal

- Beyond statistics, a new rhetoric for investment theory. 2014. Journal of Organizational Change Management

- Dynamisch ALM-beleid voor pensionfondsen. 2011. VBA-journaal

- Opkomst aandelen bij Nederlandse pensioenfondsen. 2005. IRIS Research

- Aandelen, een zaak van lange termijn. 2004. IRIS Research

- Beleggen in de BV of privé. 2003. Belastingbrief

- Klikfondsen. 2001. In handbooks 'Opties en Futures'

- Beleggen vanaf 2001: van fiscaalgedreven naar rendementgedreven. 2000. Vakblad Financiële Planning (VFP)

- Lijfrenteaftrek of zelf beleggen onder Vermeend? 2000. VFP 